\title{
Catalytic Conversion of Light Alkanes
}

\author{
Final Report \\ January 1, 1990 - October 31, 1994
}

Work Performed Under Contract No.: DE-FC21-90MC26029

For

U.S. Department of Energy

Office of Fossil Energy

Morgantown Energy Technology Center

P.O. Box 880

Morgantown, West Virginia 26507-0880

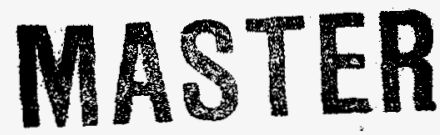

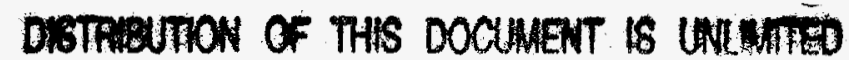

By

Sun Company, Inc.

P. O. Box 1135

Marcus Hook, Pennsylvania 19061-0835

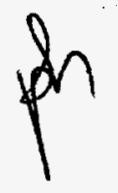




\section{Disclaimer}

This report was prepared as an account of work sponsored by an agency of the United States Government. Neither the United States Government nor any agency thereof, nor any of their employees, makes any warranty, express or implied, or assumes any legal liability or responsibility for the accuracy, completeness, or usefulness of any information, apparatus, product, or process disclosed; or represents that its use would not infringe privately owned rights. Reference herein to any specific commercial product, process, or service by trade name, trademark, manufacturer, or otherwise does not necessarily constitute or imply its endorsement, recommendation, or favoring by the United States Government or any agency thereof. The views and opinions of authors expressed herein do not necessarily state or reflect those of the United States Government or any agency thereof. 


\section{DISCLAIMER}

Portions of this document may be illegible electronic image products. Images are produced from the best available original document. 


\section{ABSTRACT}

During the course of the first three years of the Cooperative Agreement (Phase I-III), we uncovered a family of metal perhaloporphyrin complexes which had unprecedented activity for the selective air-oxidation of light alkanes to alcohols. The reactivity of light hydrocarbon substrates with air or oxygen was in the order: isobutane>propane>ethane>methane, in accord with their homolytic bond dissociation energies. Isobutane was so reactive that the proof-of concept stage of a process for producing tert-butyl alcohol from isobutane was begun (Phase V). It was proposed that as more active catalytic systems were developed (Phases IV, VI), propane, then ethane and finally methane oxidations will move into this stage (Phases VII through DX). As of this writing, however, the program has been terminated during the later stages of Phases V and VI so that further work is not anticipated.

Although active, the perhalotetraphenylporphyrin complexes are costly. Generating the perhalophenyl group at the meso position is difficult and it results in a catalyst of high molecular weight. What we learned during the first stage of the Cooperative Agreement is that the important property of a group on the periphery of the porphyrin ring is that it withdraw electrons efficiently. In Phase IV we succeeded in introducing nitro groups into the meso positions of octaethylporphyrin in a simple high-yield prep. Nitro groups are among the most electron withdrawing substituents in organic chemistry and indeed the effect of introducing them is to dramatically increase catalytic activity. Cost of the catalyst is also lower due to the ease of the synthesis and the low molecular weight of the complex.

Although improved porphine synthesis was developed and porphine nitration methods found, nitroporphines were insoluble in organic media and, therefore, impractical for homogeneous catalytic oxidations. For this reason we turned to catalysts having perfluoroalkyl groups on the porphyrin periphery as potentially inexpensive and active light alkane oxidation catalysts.

We made excellent progress during 1994 in generating a class of less costly new materials which have the potential for high catalytic activity. New routes were developed for replacing costly perfluorophenyl groups in the meso-position of metalloporphyrin catalysts with far less expensive and lower molecular weight perfluoromethyl groups. The $\mathrm{CF}_{3}$ group is more electron-withdrawing than the $\mathrm{C}_{6} \mathrm{~F}_{5}$ group and its effect on catalytic properties was of great interest. We made metalloporphyrin complexes bearing meso- $\mathrm{CF}_{3}$ groups and indeed they responded electrochemically as predicted. In addition, conceptual economic analysis indicated that these catalysts were far less expensive than our first and second generation materials. However, much like the meso-nitroporphyrins, these materials were extremely insoluble in organic media. We replaced the $\mathrm{CF}_{3}$ groups with $\mathrm{C}_{3} \mathrm{~F}_{7}$ (perfluoro n-propyl) groups and generated catalysts which had good activity and hydrocarbon solubility. With this catalyst isobutane was converted to TBA rapidly with good selectivity. The $\mathrm{C}_{3} \mathrm{~F}_{7}$ substituent was more costly to introduce than the $\mathrm{CF}_{3}$ group, however, and a still less expensive catalyst is required at this time. 
Although it is likely that catalysts not too dissimilar from those which convert isobutane to TBA could be used to convert propane to IPA, more robust catalysts will be needed for methane and ethane conversion. During the first three years of the Cooperative Agreement, we found that our first generation heterogeneous catalyst, operating between 350 and $450^{\circ} \mathrm{C}$ produced methanol and ethanol from methane and ethane. Selectivities were not high enough for a practical process, however, and detailed kinetic analysis revealed that we need to build the very same characteristics into a vapor phase catalyst as those which we use in liquid phase. Specifically, binuclear sites having high $M(\mathrm{III}) / \mathrm{M}(\mathrm{II})$ reduction potential in a hydrophobic environment are necessary. We, therefore, began a major program to design synthesize and characterize materials of this type. Considerable synthetic progress has been made and details appear in the Topical Reports, Phases IV and VI. 


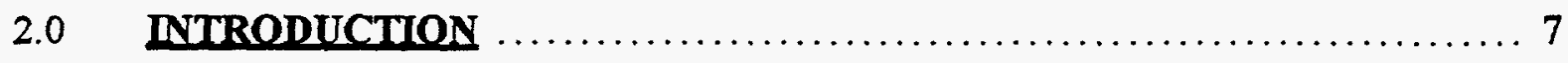

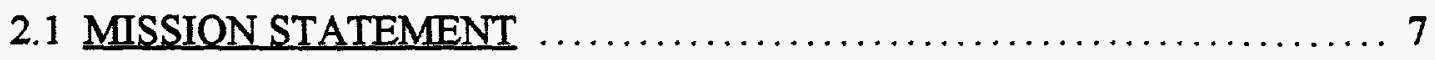

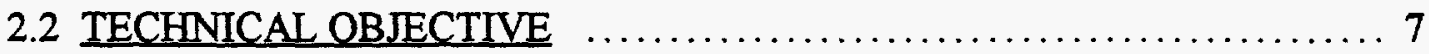

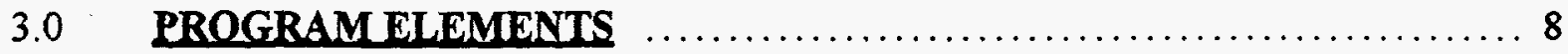

4.0 REACTION OF ALKANES WITH DIOXYGEN TOWARD SUPRABIOTIC SYSTEMS $\ldots \ldots \ldots \ldots \ldots \ldots \ldots \ldots \ldots \ldots \ldots \ldots \ldots \ldots, 10$

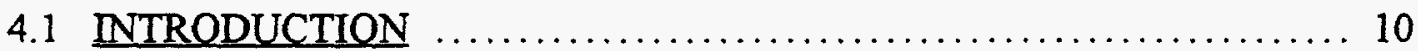

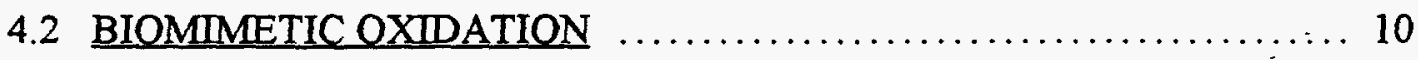

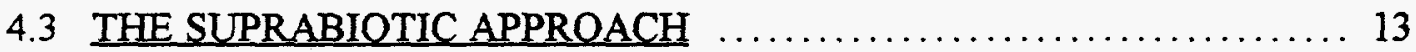

4.4 CATALYSIS BY ELECTRON DEFICIENT METALLOPORPHYRINS . 16

4.5 ELECTRON DEFICIENT METALLOPORPHYRINS AS

CATALYSTS FOR REACTIONS WITH $O_{2} \quad \ldots \ldots \ldots \ldots \ldots \ldots \ldots, 17$

4.6 FUTURE SUPRABIOTIC CATALYSTS $\ldots \ldots \ldots \ldots \ldots \ldots \ldots \ldots \ldots$

4.7 CONCLUSION $\ldots \ldots \ldots \ldots \ldots \ldots \ldots \ldots \ldots \ldots \ldots \ldots \ldots \ldots \ldots \ldots \ldots \ldots \ldots \ldots \ldots, 22$

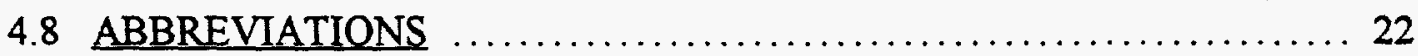

4.9 REFERENCES $\ldots \ldots \ldots \ldots \ldots \ldots \ldots \ldots \ldots \ldots \ldots \ldots \ldots \ldots \ldots \ldots \ldots \ldots \ldots \ldots \ldots \ldots, 22$

5.0 AZIDE ACTIVATION OE METALLOPHTHALOCYANINE COMPLEXES FOR THE CATALYTIC OXIDATION OF ALKANES IN THE LIOUID PHASE $\ldots \ldots \ldots \ldots \ldots \ldots \ldots \ldots \ldots \ldots \ldots \ldots \ldots, 43$

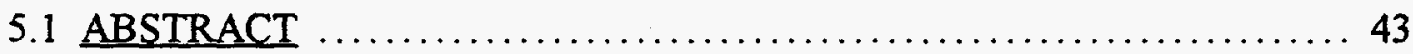

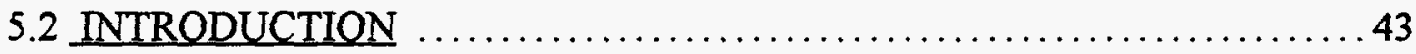




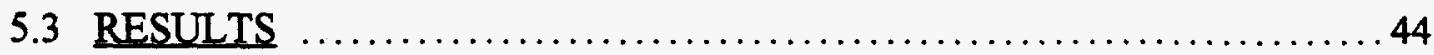

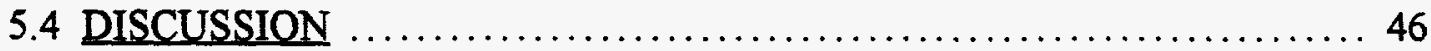

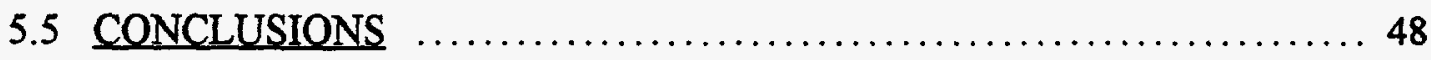

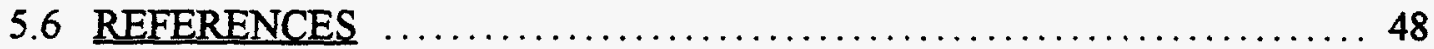

6.0 HALOGEN SUBSTITUENT EEFECTS ON THE CATALYTIC ACTIVITY OE IRON PORPHYRIN COMPLEXES FOR THE DECOMPOSITION

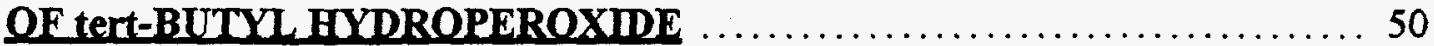

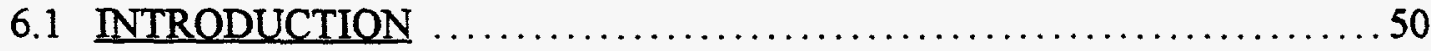

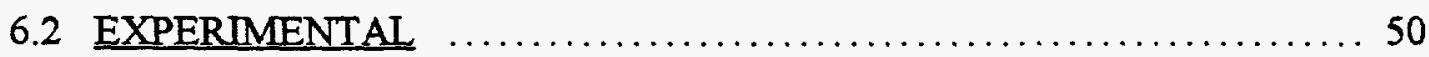

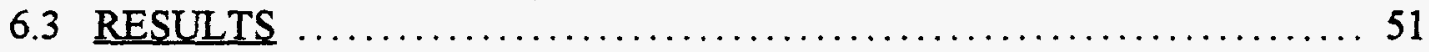

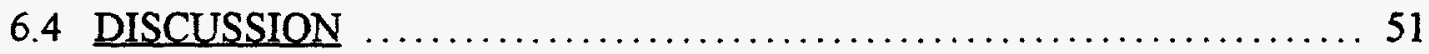

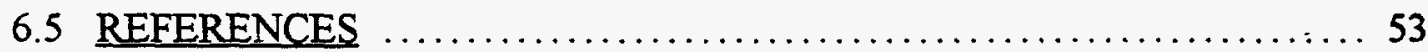

7.0 HALOGENATED METALLOPORPHYRIN COMPLEXES AS CATALYSTS FOR SELECTIVE REACTIONS OF ACYCLIC ALKANES WITH MOLECULAR OXYGEN

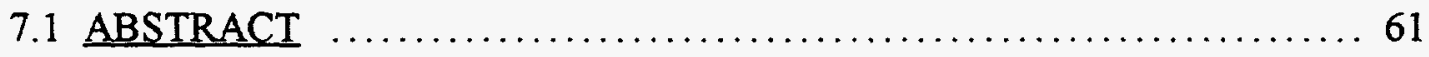

7.2 INTRODUCTION $\ldots \ldots \ldots \ldots \ldots \ldots \ldots \ldots \ldots \ldots \ldots \ldots \ldots \ldots \ldots, 61$

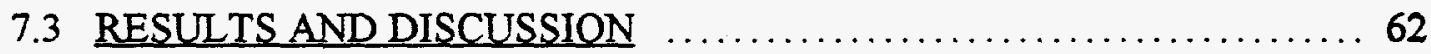

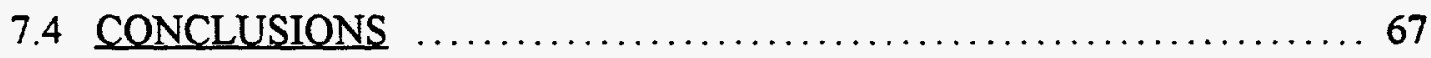

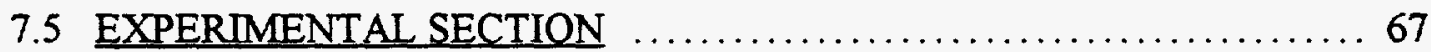

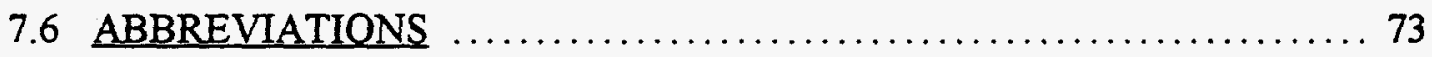

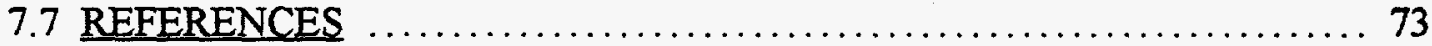

8.0 PERELUOROALKYLPORPHYRIN COMPLEXES AS ACTIVE CATALYSTS FOR THE REACTION OF ISOBUTANE WITH OXYGEN AND THE DECOMPOSITION OE tert-BUTLL HYDROPEROXIDE $\ldots 100$

8.1 ABSTRACT 100 


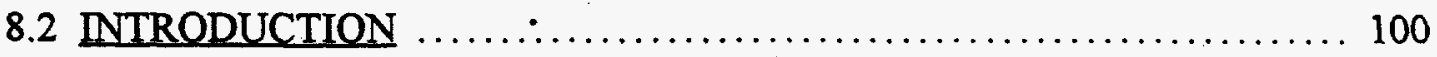

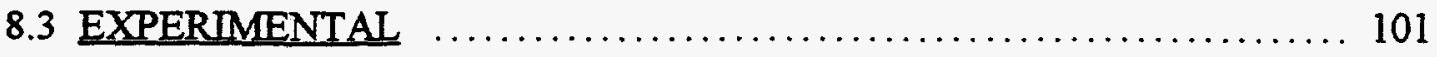

8.4 RESULTS AND DISCUSSION $\ldots \ldots \ldots \ldots \ldots \ldots \ldots \ldots \ldots \ldots \ldots \ldots \ldots$

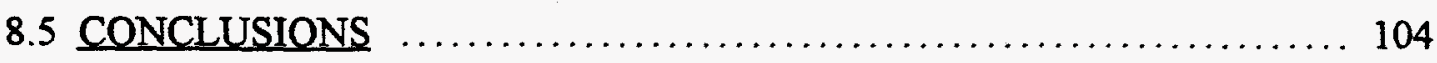

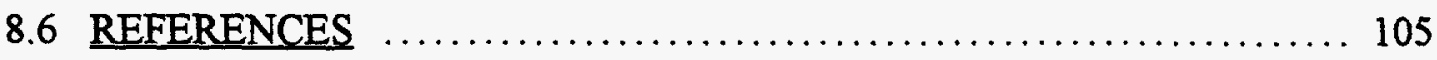

9.0 mESO-PERELUOROALKYL DIPYRROMETHANES AND PORPHYRINS

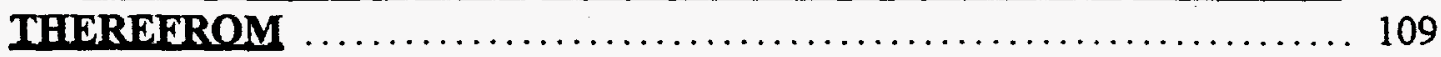

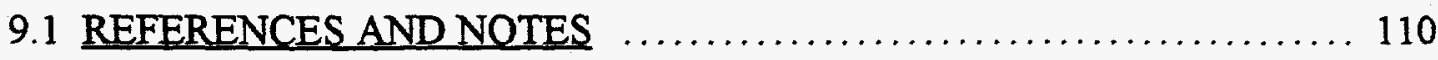

10.0 NOVEL IRON meSO-NITROOCTAETHYLPORPHINE COMPLEXES FOR THE CATALYTIC REACTION OF ALKANES WITH MOLECULAR OXYGEN

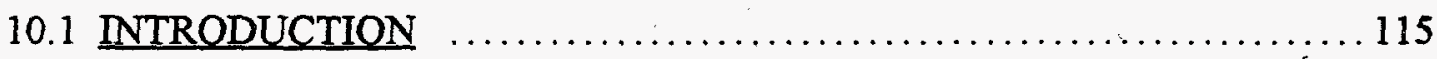

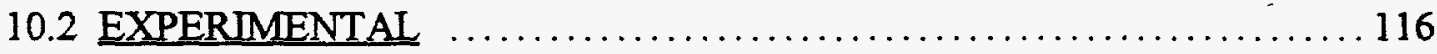

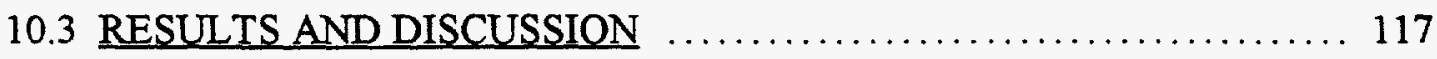

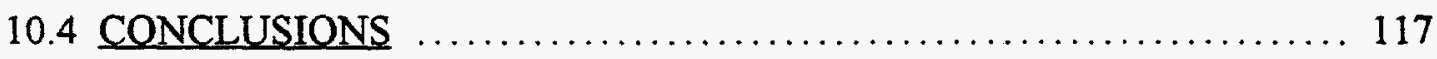

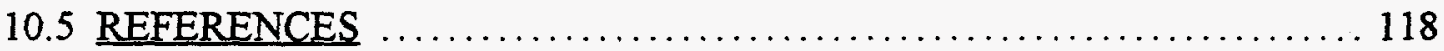

11.0 CATALYTIC CONVERSION OF LIGHT ALKANES - PROOF OF CONCEPT STAGE 121

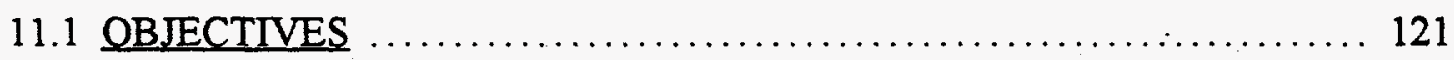

11.2 BACKGROUND INFORMATION $\ldots \ldots \ldots \ldots \ldots \ldots \ldots \ldots \ldots \ldots \ldots \ldots \ldots \ldots \ldots \ldots \ldots, 121$

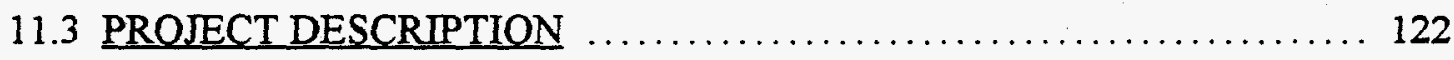

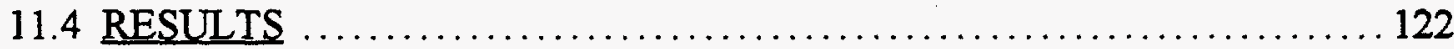

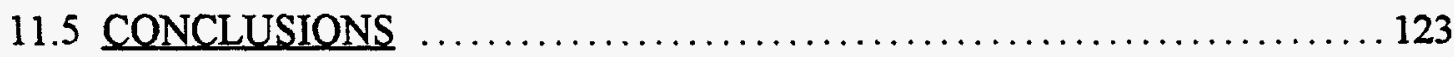

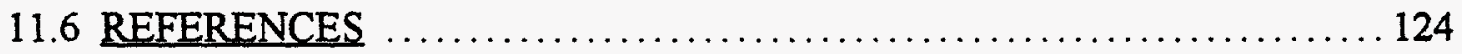


12.1 SUMMARY

12.1.1 Methane Oxidation Kinetic Model

12.1.2 Catalyst Synthesis Characterization and Testing

12.2.1 Iron Sodalite

12.2.2 Metals In Regular Oxidic Matrices $\ldots \ldots \ldots \ldots \ldots \ldots \ldots \ldots \ldots \ldots \ldots \ldots$

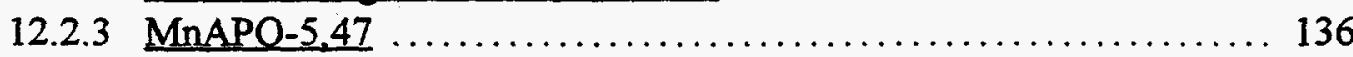

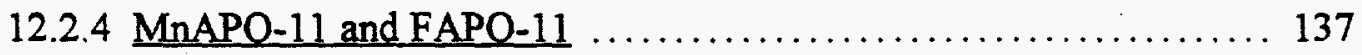

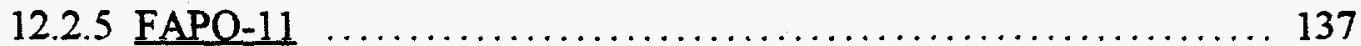

12.2.6 $\mathrm{MnAPO}-11$

12.3 CATALYST TESTING $\ldots \ldots \ldots \ldots \ldots \ldots \ldots \ldots \ldots \ldots \ldots \ldots \ldots \ldots \ldots \ldots \ldots \ldots, 140$

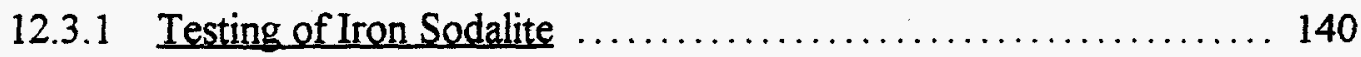

12.3.2 Effects of Reactor Design on Methane Oxidation .............. 145

12.3.3 Catalytic testing in a Downflow Reactor ................... 154

12.3.4 Oxidation of Ethane Over Fex[Fe]SOD $\quad \ldots \ldots \ldots \ldots \ldots \ldots \ldots \ldots$

12.3.5 Formaldehyde Analysis $\ldots \ldots \ldots \ldots \ldots \ldots \ldots \ldots \ldots \ldots \ldots \ldots \ldots$

12.3.6 Oxidation of Methane Over MnAPO-X $\ldots \ldots \ldots \ldots \ldots \ldots \ldots 158$

12.3.7 Oxidation of Ethane and Methane Over Framework Substituted Zeolites 158

12.3.8 Methane and Ethane Oxidations Over MnAPO-5 ........... 166

12.3.9 Methane Oxidation Over [Fe]ZSM-5 . .................... 169

12.3 .10 Reactions catalyzed by $0.5 \% \mathrm{CrQ}_{3} \mathrm{SiO}_{2} \ldots \ldots \ldots \ldots \ldots \ldots . \ldots 169$

12.3.11 Catalyst Testing in a CSTR .......................... 169

12.3.12 Methane Oxidations Under Different Conditions ............. 176

12.3.13 Experimental Details of Methane Oxidations $\ldots \ldots \ldots \ldots \ldots \ldots . . \ldots 176$

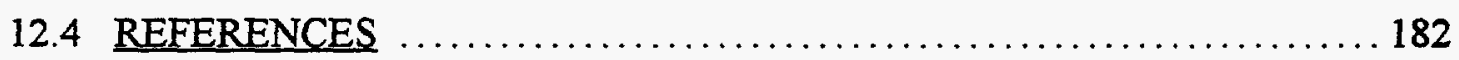

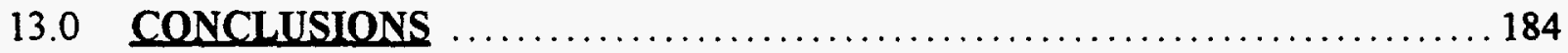


13.1 THE SUPRABIOTIC CONCEPT 184

13.2 MOLECULAR COMPLEXES FOR HOMOGENEOUS LIOUID PHASE CONVERSIONS 184

13.3 FRAMEWORK SUBSTITUTED ZEOLITES 189

13.4 CATALYST SYNTHESIS AND TESTING 190

14.0 SUN PATENTS AND PUBLICATIONS DURING THE COURSE OF THE COOPERATIVE AGREEMENT

14.1 PATENTS ISSUED OR PUBLISHED 194 14.2 PUBLICATIONS 196 


\section{LIST OF TABLES}

\section{CHAPTER 1}

Table 1-1 Properties of Ethers and Alcohols

\section{CHAPTER 3}

Table 3.1 Components of New Coopeative Agreement

\section{CHAPTER 4}

Table 1 Oxidation of Isobutane Catalyzed by Metalloporphyrin Azide and Nitride Complexes ${ }^{2}$

Table 2 Oxidation of Isobutane Catalyzed by Fluorinated Metalloporphyrin Complexes ${ }^{2}$

Table 3 Isobutane Oxidations Catalyzed by Perhaloporphyrin Complexes

Table 4 Propane Oxidations Using First Row Metal Complexes of TPPF ${ }_{20} \beta-\mathrm{Br}_{8}$ as Catalysts ${ }^{2}$

Table 5 Iron Haloporphyrin Catalyzed Isobutane Oxidation ${ }^{2}$

Table 6 Alkane Oxidations Catalyzed by Iron Porphyrins, $\mathrm{Fe}\left(\mathrm{TPPZ}_{20} \beta-\mathrm{Y}_{8}\right) \mathrm{X}^{\mathrm{ab}}$

Table 7 Relationship Between Catalyst Reduction Potential and Activity

Table 8 oValues for Common Functional Groups

\section{CHAPTER 5}

Table 1 Oxidation of Isobutane $e^{a}$ Catalyzed by Metal Phthalocyanine Azides

Table 2 Oxidation of Propane ${ }^{a}$ Catalyzed by an Iron Perfluorophthalocyanine Azide Complex

\section{CHAPTER 6}

Table 1 Conversion of tert-Butyl Hydroperoxide to tert-Butyl Alcohol

\section{CHAPTER 7}

Table 1 Effect of Fluorination of The meso-Phenyl Groups on the Activity of Tetraphenyporphyrinato Metal Complexes for Reactions of Isobutane with Oxygen ${ }^{a}$

Table 2 Effect of Ring Halogenation on the Isobutane Oxidation Activity of Porphyrinatoiron(III) Complexes ${ }^{2}$

Table 3 Isobutane Oxidations Catalyzed by Perhaloporphyrin Complexes ${ }^{a}$

Table 4 Propane Oxidations Using First Row Metal Complexes of $\mathrm{TPPF}_{20} \beta-\mathrm{Br}_{8}$ as Catalysts ${ }^{\mathrm{a}}$

Table 5 Alkane Oxidations Catalyzed by Cobalt Complexes 


\section{LIST OF TABLES}

(Cont'd)

CHAPTER 7 (Cont'd)

Table 6 Iron Haloporphyrin Catalyzed Isobutane Oxidation ${ }^{2}$

Table 7 Conversion of tert-Butyl Hydroperoxide to tert-Butyl Alcohol

Table 8 Relationship Between Catalyst Reduction Potential and Hydroperoxide Decomposition Activity

Table 9 n-Butane Oxidations Catalyzed By TPPF ${ }_{20} \beta-\mathrm{Cl}_{8}$ Complexes of Iron and Chromium

Table 10 Alkane Oxidations Catalyzed by Iron Halotetraphenylporphyrins ${ }^{\star}$

Table 11 Comparison of the Product Profile of 2-Methylpentane Oxidation with that of tertAmyl Hydroperoxide Decomposition Catalyzed by Perhaloporphyrinato Iron Complexes

Table 12 Product Profile as a Function of $\mathrm{R}$ in Oxidation of $\mathrm{R}_{1} \mathrm{R}_{2} \mathrm{R}_{3} \mathrm{CH}$ Catalyzed by $\mathrm{Fe}\left(\mathrm{TPPF}_{20} \beta-\mathrm{BR}_{8}\right) \mathrm{Cl}^{\mathrm{a}}$

CHAPTER 8

Table 1 Effect of meso-perfluoroalkyl Substituents on the catalytic activity of electron deficient iron porphyrin complexes

Table 2 Effect of $\beta$-Bromination of $\mathrm{III}$ and $\mathrm{V}$ on Catalytic Isobutane Oxidation Activity

CHAPTER 10

Table 1 Isobutane Oxidation Activity of Iron Porphyrin Complexes

\section{CHAPTER 11}

Table 1 Iron Haloporphyrin - Catalyzed Isobutane Oxidation

Table 2 Conversion of TBHP to TBA

Table 3 Electron Affinity, Reduction Potential and Catalytic Activity

Table 4 Isobutane to TBA Proof-of-Concept Criteria (New Retrofit Process Modes)

\section{CHAPTER 12}

Table 1 Iron Sodalite X-Ray Diffraction Pattern

Table 2 Vapor Phase Air Oxidations of Methane to Methanol

Table 3 Effect of By-pass in Methane Oxidation Over Fe[SOD] in Quartz Lined Tubular Reactor

Table 4 Oxidations of Ethane Over $\mathrm{Fe}_{x}[\mathrm{Fe}] \mathrm{SOD}^{\star}$

Table 5 Methane Oxidations

Table 6 Ethane Oxidations

Table 7 Methane Oxidations 


\section{LIST OF TABLES}

(Cont'd)

Table 8 Methane Oxidations Over MnAPO-5 and Fex[Fe]SOD

Table 9 Ethane Oxidations Catalyzed By Fe ${ }_{x}$ [Fe]SOD or MnAPO-5

Table 10 Oxidation of Methane Over Fe[ZSM-5 $]^{b}$ in the Vapor Phase

\section{CHAPTER 12}

Table 11 Oxidation of Methane Over Chromia on Silica ${ }^{a}$

Table 12 Methane Oxidation in a CSTR

Table 13 Comparison of an Activated [Fe]SOD and Silica Gel in a CSTR Reactor at $400^{\circ} \mathrm{C}$ Under Fully Backmixed Conditions with the Bypass Open

Table 14 Oxidations of Methane at High $\mathrm{O}_{2}$ and GHSV

Table 15 Effect of Pressure on Methane Oxidations Catalyzed by Fe[SOD]in a Kung Reactor ${ }^{2}$ 


\section{LIST OF FIGURES}

\section{CHAPTER 1}

Figure 1-1 Relative Cost of Transport of Liquid and Gaseous Fuels (LNG = liquified natural gas).

Figure 1-2 Hydrocarbon Reactivity Series

\section{CHAPTER 4}

Figure 1 Biological and Biomimetic Alkane Oxidation

Figure 2 Hydrogen as a Co-Reductant in a Biomimetic Oxidation

Figure 3 Alkane Hydroxylation with $\mathrm{O}_{2}$ Using a Suprabiotic Catalyst

Figure 4 Properties of a Suprabiotic Catalyst

Figure 5 Substituted Porphyrin Macrocycle

Figure 6 Catalyst Activity vs. Redox Potential

\section{CHAPTER 6}

Figure 1 Formation of tert-butyl alcohol, TBA, from tert-butyl hydroperoxide $(10 \mathrm{ml})$ in benzene $(48 \mathrm{ml})$-p-xylene $(2.4 \mathrm{ml})$ using $2 \times 10^{-4}$ mmoles of catalyst:

$\circ \mathrm{Fe}\left(\mathrm{TPPF}_{20} \mathrm{\beta}-\mathrm{Cl}_{8}\right) \mathrm{Cl}, \triangle \mathrm{Fe}\left(\mathrm{TPPF}_{20}\right) \mathrm{Cl}$, $\mathrm{Fe}(\mathrm{TPP}) \mathrm{Cl}, \wedge \mathrm{Fe}(\mathrm{acac})_{3}$.

Figure 2 Second order plots for the catalytic decomposition of tert-butyl hydroperoxide in benzene $\diamond \mathrm{Fe}\left(\mathrm{TPPF}_{20} \mathrm{\beta}-\mathrm{Cl}_{8}\right) \mathrm{Cl}, \triangle \mathrm{Fe}\left(\mathrm{TPPF}_{20}\right) \mathrm{Cl}, \quad \mathrm{OFe}(\mathrm{TPP}) \mathrm{Cl}$.

Figure 3 Effect of BHT on the decomposition of tert-butyl hydroperoxide $(10 \mathrm{ml})$ catalyzed by $\mathrm{Fe}\left(\mathrm{TPPF}_{20} \beta-\mathrm{Cl}_{8}\right) \mathrm{Cl}, 2 \times 10^{-4}$, mmoles, in benzene $(48 \mathrm{ml})$ - $\mathrm{p}$-xylene $(2.4 \mathrm{ml})$.

Scheme 1 Haber-Weiss decomposition of hydroperoxides using metalloporphyrins as catalysts (porphyrin ring omitted).

Scheme 2 Possible metal oxo pathways for decomposition of hydroperoxides using metalloporphyrin catalysts (porphyrin ring omitted)

\section{CHAPTER 7}

Figure 1 The Substituted Porphyrinato Ligand; $\mathrm{Y}=\mathrm{-C} 1, \mathrm{Br}, \mathrm{orH} ; \mathrm{Z}=\mathrm{C}_{6} \mathrm{H}_{5}(\mathrm{TPP})$, Q- $\mathrm{C}_{6} \mathrm{H}_{4} \mathrm{Cl}_{2}\left(\mathrm{TPPCl}_{8}\right)$ or $\mathrm{C}_{6} \mathrm{~F}_{5}\left(\mathrm{TPPF}_{20}\right)$.

Figure 2 Oxidation of Isobutane in Benzene at $80^{\circ} \mathrm{C}$ using $\mathrm{Fe}\left(\mathrm{TPPF}_{20}\right) \mathrm{X} ; \mathrm{X}=\mathrm{Cl}, \bullet, \mathrm{N}_{3}, \bullet$, and $\mathrm{OH},{ }^{\circ}$ as axial ligands. 


\section{LIST OF FIGURES}

(Cont'd)

CHAPTER 7 (Cont'd)

Figure 3 Catalyst Turnovers as a function of $\mathrm{Fe}(\mathrm{III}) / \mathrm{Fe}(\mathrm{II})$ Oxidation Potential. -o- Isobutane Oxidations at $60^{\circ} \mathrm{C} ;-0$ - Propane Oxidations at $125^{\circ} \mathrm{C}$. See Tables 3,4 for experimental procedures. 1, Fe(TPP)OH; $2 \mathrm{Fe}\left(\mathrm{TPPCl}_{8}-\mathrm{Br}_{4}\right) \mathrm{OH}$; 3, $\mathrm{Fe}\left(\mathrm{TPPF}_{20}\right) \mathrm{OH}$; 4, $\mathrm{Fe}\left(\mathrm{TPPF}_{20}-\mathrm{Br}_{8}\right) \mathrm{OH} ; 5, \mathrm{Fe}\left(\mathrm{TPPF}_{20}-\mathrm{Cl}_{8}\right) \mathrm{OH}$.

Figure 4 Schematic representation of biological and biomimetic alkane oxidation (40-47)

Figure 5 Conceptual scheme for a hypothetical catalytic cycle for conversion of an alkane to an alcohol using an electron deficient iron porphyrin complex (porphyrin ring omitted).

Figure 6 Formation of tert-butyl alcohol, TBA, from tert-butyl hydroperoxide $(10 \mathrm{ml})$ in benzene ( $48 \mathrm{ml}$ )-p-xylene ( $2.4 \mathrm{ml})$ using 2 x 10-4 mmoles of catalyst: $O$ $\mathrm{Fe}\left(\mathrm{TPPF}_{20}-\mathrm{Cl} \mathrm{l}_{8} \mathrm{Cl}, \square \mathrm{Fe}\left(\mathrm{TPPF}_{20}\right) \mathrm{Cl}, \bullet \mathrm{Fe}(\mathrm{TPP}) \mathrm{Cl}, \boldsymbol{\mathrm { Fe }}(\mathrm{acac}) 3\right.$.

Figure 7 Haber-Weiss decomposition of hydroperoxides using metalloporphyrins as catalysts (porphyrin ring omitted).

Figure 8 Oxidation of n-butane in acetonitrile at $125^{\circ} \mathrm{C}$ catalyzed by $\operatorname{Cr}\left(\mathrm{TPPF}_{20}-\mathrm{Cl}_{8}\right) \mathrm{N}_{3}$. Details given in Table 10.

Figure 9 Dependence of product profile on substrate structure for alkane oxidations catalyzed by $\mathrm{Fe}\left(\mathrm{TPPF}_{20} \mathrm{\beta}-\mathrm{Br}_{8}\right) \mathrm{OH}$.

Figure 10 Possible pathways occuring during selective oxidation of alkanes catalyzed by iron perhaloporphyrin complexes. a) hypothetical alkoxy radical.

\section{CHAPTER 8}

Figure 1 The substituted porphyrinato ring system: meso-substituents $=\mathrm{C}_{6} \mathrm{H}_{5}, \mathrm{C}_{6} \mathrm{~F}_{5}, \mathrm{CF}_{3}, \mathrm{C}_{3} \mathrm{~F}_{7}$; $\beta$-groups $=\mathrm{H}, \mathrm{Br}$; axial ligand $=\mathrm{OH}, \mu$-oxo $(\mathrm{PFeOFeP})$

CHAPTER 10

Figure 1 The meso-Tetranitrooctaethylporphinato Ligand 


\section{LIST OF FIGURES}

(Cont'd)

\section{CHAPTER 11}

Figure 1 Catalyst Activity vs. Redox Potential

Figure 2 TBA from TBHP

\section{CHAPTER 11}

Figure 3 Isobutane Oxidation - One Step Process

Figure 4 Isobutane Oxidation - Two Step Process

\section{CHAPTER 12}

Figure 1 MnAPO47As Synthesized

Figure 2 MnAPO47 Calcined

Figure 3 XRD Spectrum of FAPO-11

Figure 4 XRD Spectrum of MnAPO-11

Figure 5 Light Alkanes to Liquid Octane Methane to Methanol

Figure 6 Superimposed PXRD's of Fresh and Used [Fe]Sodalite

Figure 7 Temperature and Space Velocity Dependence of Methanol Selectivity in Quartz Bypass Reactor

Figure 8 Hypothetical Catalytic Cycle for Silicoferrate Activity

Figure 9 Quartz Lined Bypass Reactor System

Figure 10 Plug Flow Operation

Figure 11 Cstr with Robinson-Mahoney Internals and External Bypass

Figure 12 Methane Oxidations

Figure 13 Formaldehyde Analysis of (High Formaldehyde Level)

Figure 14 Formaldehyde Analysis of (Low Formaldehyde Level)

Figure 15 Formaldehyde Analysis of (Low Formaldehyde Level)

Figure 16 Formaldehyde Analysis of (Low Formaldehyde Level)

Figure 17 CSTR Basket Reactor

Figure 18 P Dependence of $\mathrm{CH}_{3} \mathrm{OH}$ Yield

Figure $19 \quad \mathrm{CH}_{3} \mathrm{OH}$ Yield vs. $\mathrm{CH}_{2} \mathrm{O}$ Yield

CHAPTER 13

Figure 1 Hypothetical Reaction Pathway for a Suprabiotic Catalytic Alkane Oxidation

Figure 2 Properties of a Suprabiotic Catalyst

Figure 3 Proposed Mechanism for MMO Activity

Figure 4 Schematic Binuclear Suprabiotic Catalytic Alkane Activation 


\subsection{SUMMARY OF PROGRAM INCENTIVES}

As petroleum reserves dwindle, all hydrocarbon raw materials which could be converted to usable liquid fuels become more dear. Often the search for oil yields extensive gas reservoirs. Gas availability on a worldwide scale rivals that of crude oil but problems of transportation and differences in end use application often limit its' utility. Most natural gas is found in areas remote to its ultimate use. Currently much of this remote gas is associated with petroleum production. Because it cannot be used at the location where it is produced, it is often flared on a large scale, representing not only a wasted energy resource but also contributing to global warming as a result of carbon dioxide production. Because of its low energy density, methane is relatively costly to transport. Figure 1-1 shows the relative differences between the costs of transporting various liquid and gaseous fuels $(1)$. It is readily seen that the conversion of methane to a liquid fuel such as methanol or a hydrocarbon liquid would both drastically cut the cost of transport of over long distances, and provide an alternative fuel liquid from an abundant, under-utilized resource.

Catalytic alkane conversion science is maturing to the point that one can envision the economically attractive conversion of natural gas to liquid fuels on a commercial scale by the turn of the century. This situation has many international and national implications. Remote accumulations of low value gas such as exist on the North slope of Alaska and various off-shore sites could be exploited. Value could be maximized for domestic gas reserves in high value-added products. The National interests would be well served if the U.S. held the technological lead in gas conversion technology and could influence the course of gas conversion abroad. One of the most costly steps in the current technology for converting natural gas to liquid fuels is the energy-intensive steam reforming step. The products of steam reforming are catalytically converted to methanol which has value as an alternative liquid fuel product or which could be converted to gasoline via existing technology.

We have examined a new catalytic approach to the direct production of a methanol-rich oxidate from natural gas which avoids costly steam reforming completely. Work conducted in Sun laboratories under the joint sponsorship of the Department of Energy (METC), the Gas Research Institute, and the Sun Company, Inc., has produced a family of new catalytic materials which, if successfully developed, will be effective in the conversion of light alkanes to alcohols or other oxygenates. We have found that it is possible to catalytically convert methane, and the $\mathrm{C}_{2}-\mathrm{C}_{4}$ hydrocarbons that are present in smaller but significant amounts in natural gas, to alcohols by direct reaction with air or oxygen. In particular, we have found that the $\mathrm{C}_{4}$ component of natural gas can be catalytically converted to fuel oxygenates in excellent yield, and significant progress has been made in converting the $\mathrm{C}_{3}$ component to an oxygenate product without combusting the hydrocarbon substantially. The $C_{1}$ and $C_{2}$ components can also be converted to alcohol-rich oxygenates but more progress is still required before a practical catalytic route becomes possible. 


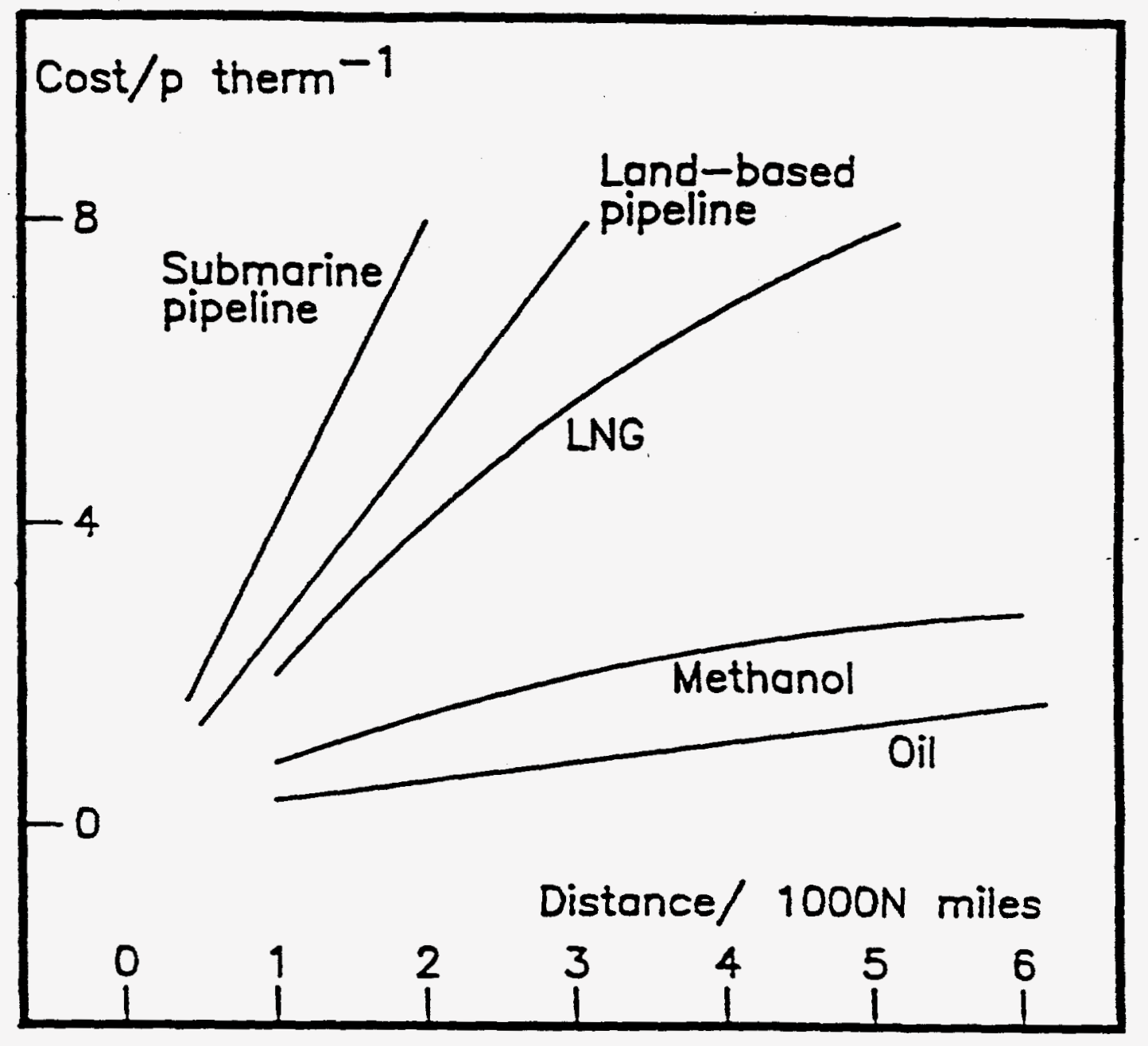

FIGURE 1.1 Relative cost of transport of liquid and gaseous fuels (LNG = liquified natural gas). 
At the outset of this work we envisioned converting natural gas to an alcohol-rich oxidate which could subsequently be converted to hydrocarbon gasoline via MTG technology. During the period of this research, however, the benefits of oxygenates as clean-burning, high octane, liquid fuel materials has become apparent. Alcohols and the ethers that can be made from them are the oxygenates that are used to replace aromatics in the reformulated gasolines of the 90 's, reduce toxicity and reduce ozone and carbon monoxide. As a result, it is more reasonable to consider the direct air-oxidation of light alkanes to produce alcohol-rich oxidates for use as components of reformulated gasoline or as liquid fuel alternatives to gasoline in areas where there is non-attainment of $\mathrm{CO}$ or ozone limits set forward by the Clean Air Act. Alcohols of the type that can be formed directly from natural gas components: methanol, ethanol and tert-butyl alcohol, TBA, are not only useful as liquid fuel components themselves but are used in the manufacture of ether oxygenates such as MTBE, TAME, and ETBE as well. Table 1-1 shows some of the fuel-related properties of the $C_{1}-C_{4}$ alcohols and the ethers that can be made from them. It can be seen that these materials have desireable fuel properties in addition to meeting the fuel oxygen-content requirements of the Clean Air Act.

As desireable as it is to convert light alkanes directly to alcohols, doing this in an efficient manner using existing technology is a major problem. The light alkanes: methane, ethane, propane and the butanes, are among the most unreactive of organic substrates. They are also among the most abundant and inexpensive raw materials. They are found not only in natural gas, but in light petroleum fractions, and are being generated in ever-increasing quantities in petroleum refineries due to the increasing severity of catalytic cracking of heavier crudes. There exist few commercial processes for the efficient upgrading of these substrates to high value products and no DIRECT catalytic functionalization of these substrates to produce chemical or fuel alcohols.

During a six-year period in which Sun has invested over six million dollars in research support, coupled with an ongoing program sponsored jointly by Sun, the DOE and GRI, we have formulated and demonstrated a novel catalyst concept which allows the low temperature liquid or vapor phase catalytic conversion of light alkanes, $\left(\mathrm{C}_{1}-\mathrm{C}_{4}\right)$, to their respective alcohols or ketones. This concept grew out of an understanding of biomimetic systems, together with new inorganic and catalytic chemistry. It is known that high oxidation state metal oxo $(M=0)$ complexes will react with alkanes to give alcohols under mild conditions. Iron oxo $(\mathrm{Fe}=\mathrm{O})$ intermediates are, no doubt, involved in the enzymatic hydroxylation of $\mathrm{C}-\mathrm{H}$ bonds in vivo (Cytochrome $\mathrm{P}-450$ ). To date no one has been able to synthesize these $\mathrm{Fe}=\mathrm{O}$ species under mild conditions using ONLY molecular oxygen as oxidant. Even nature requires the stoichiometric consumption of a co-reductant which donates the electrons and protons needed for closing the catalytic oxidation cycle. Nature's co-reductant is NADH which is converted to NAD while donating electrons and proton. Synthetic biomimetic systems add stoichiometric reagents for providing two protons and two electrons for every hydrocarbon molecule which is oxidized. These reagents are regenerated in*vivo but, in vitro processes require sacrificial consumption of valuable raw materials in the regeneration process. This stoichiometric requirement imposes severe and usually prohibitive economic constraints on commercial applications of such a catalytic process. 
TABLE 1-1

\section{PROPERTIES OF ETHERS AND ALCOHOLS}

$\begin{array}{lcccccc}\text { PROPERTY } & \text { MTBE } & \text { ETBE } & \text { GTBA } & \text { IPA } & \text { ETOH } & \text { MeOH } \\ \text { Oxygen, wt \% } & 18.2 & 15.7 & 21.6 & 26.6 & 34 & 49.9 \\ \text { Sp. Gravity } & 0.74 & 0.74 & 0.791 & 0.789 & 0.79 & 0.796 \\ \text { Boiling Point, } F & 131 & 163 & 181 & 180 & 172 & 149 \\ \text { RVP (Ibs/in }{ }^{2} \text { ) } & 7.8 & 2.5 & 1.8 & 1.8 & 2.3 & 4.6 \\ \text { Blending RVP } & 9 & 5 & 12 & 14 & 23 & 75 \\ \text { RON BV } & 110-122 & 117-121 & \sim 103 & 113-119 & \sim 122 & 126 \\ \text { MON BV } & 98-102 & 100-105 & \sim 91 & 95-101 & \sim 96 & 104 \\ (\mathrm{R}+\mathrm{M}) / 2 \text { BV } & 103-110 & \sim 110 & \sim 97 & 104-110 & \sim 100 & 115\end{array}$


In our laboratories we have learned how to tune the redox potential of metals in macrocyclic ligand environments to make them active for the selective air-oxidation of alkanes using ONLY molecular oxygen. As we mentioned above, nature uses electrons and protons together with iron to split the oxygen molecule and generate $\mathrm{Fe}=0$. In so doing half of the oxygen molecule is converted to water while the other half is useful in oxidizing a substrate. We have asked the question whether oxygen can be split between two $\mathrm{Fe}$ (II) centers to make two $\mathrm{Fe}=\mathrm{O}$ species using all of the oxygen as effective oxidant. While Cytochrome P-450 and MMO act as monooxygenases, we are making complexes which may act as synthetic dioxygenases. We have incorporated a number of oxidation-active metal centers into our synthetic catalysts in addition to iron and have found that we are able to convert methane, ethane, propane and the butanes either to the corresponding alcohol or carbonyl compound depending on the nature of the metal center in the macrocyclic ligand system. All-inorganic "macrocycles" such as polyoxometallates and framework substituted zeolites have also been investigated for both liquid and vapor phase catalytic oxidations.

The ease of oxidation of the substrates that we have studied are in the order isobutane> butane>propane>ethane>methane, which is also the order of increasing $\mathrm{C}-\mathrm{H}$ bond dissociation energy, ${ }^{*}$ Figure 1-2. Isobutane is the easiest of the light hydrocarbons to oxidize. As a result we are close to having achieved a practical catalytic process for isobutane oxidation. High rates, yields and selectivities have been observed. For this reason, we proceeded with the proof-of-concept phase for a process that converts isobutane to tert-butyl alcohol. There are many reasons why such a process is of interest. First, sales of field butanes from the gas industry to refiners is down due to the vapor pressure restrictions on automotive transportation fuels. Conversion of C4's to TBA would be a way to put unwanted butanes back into gasoline. TBA is both a commercially tested high octane, clean burning motor fuel component and in addition is the precursor to MTBE, a rapidly growing commodity fuel material which is being used to meet oxygen mandates of the Clean Air Act.

After successful completion of a three year research program, a four year program of work was undertaken to proceed further which was planned to involve over 500 man-months of chemist, engineer and technician manpower for a duration of four years at a total cost of approximately $12 \mathrm{MM}$ dollars and involve "proof of concept" activities. Work was interrupted during the second year of the advanced effect. 


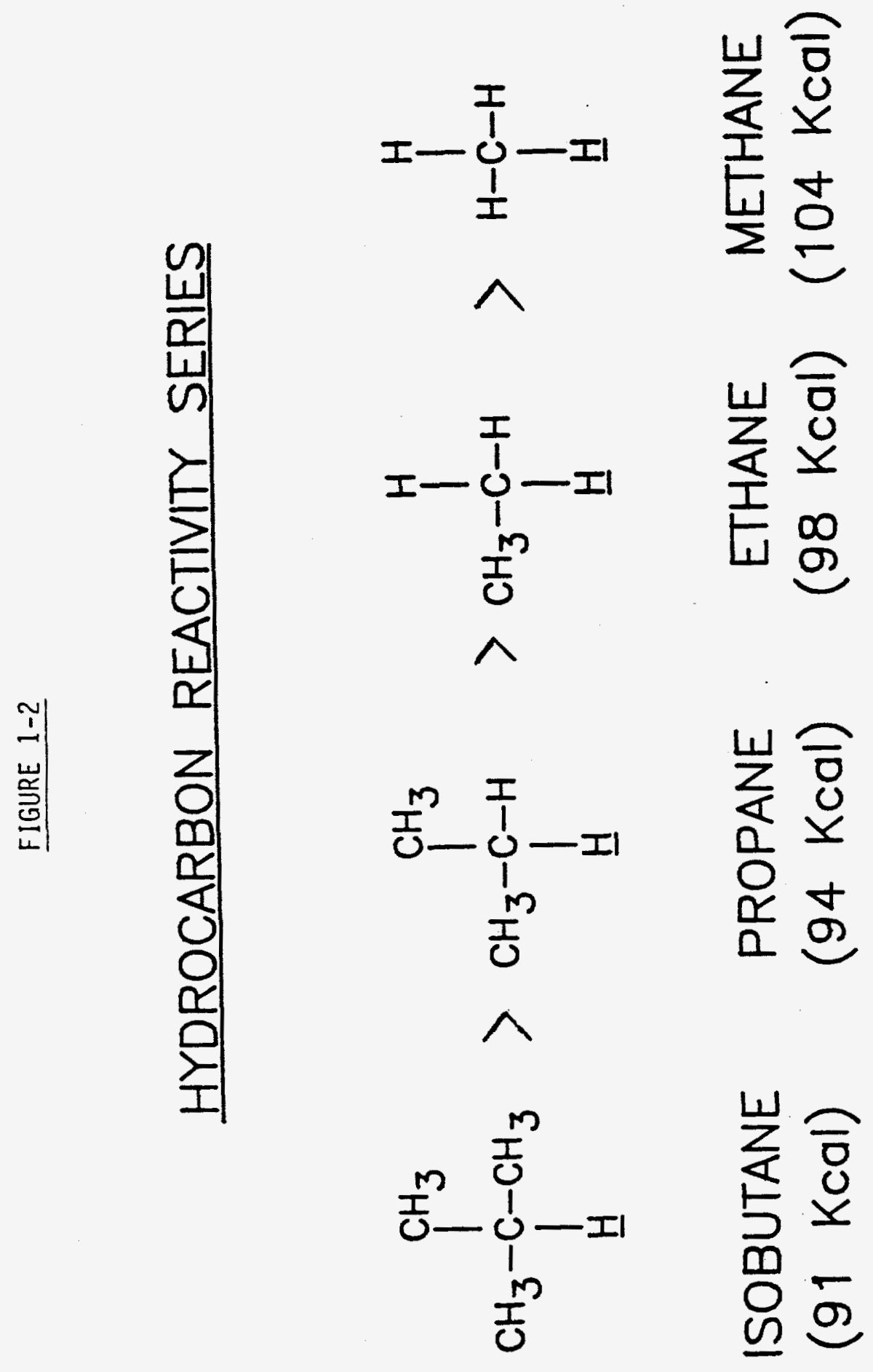




\subsection{MISSION STATEMENT}

The mission of the work presented in this proposal is to generate novel catalytic technology which will permit the development of a simple, efficient and economical process for the direct conversion of natural gas to liquid transportation fuels. This process should be simple enough to liquify natural gas economically even at a remote reservoir site.

The most costly and energy-intensive step in currently applied commercial technology for the conversion of natural gas to liquid fuels is the steam reforming of the light hydrocarbons to produce synthesis gas. The synthesis gas is then converted to methanol which is passed over a catalyst to produce gasoline. If it were possible to catalytically transform natural gas into a methanol rich-oxidate rapidly and efficiently in a single step using air or $\mathrm{O}_{2}$ as oxidant, the economics of the process would be dramatically improved (1-4). In contrast to the energy-inefficient steam reforming process, a direct "methane-to-methanol" process would be highly exoergic and, if properly carried out, would generate usable energy as steam, electricity or both. For these reasons, we are attempting to develop a process for the direct conversion of natural gas to an environmentally superior, methanol-rich liquid fuel oxygenate.

\subsection{TECHNICAL OBJECTIVE}

The technical objective of this proposal is to synthesize the first effective, practical molecular catalyst and demonstrate its use for the direct air-oxidation of natural gas to a methanol-rich oxidate which can either be used directly or converted into gasoline via known technology.

The light alkanes found in natural gas - methane, ethane, propane, and butane are among the most unreactive of all organic substrates. No effective catalysts are known for the selective partial oxidation of these relatively refractory materials. The development of an efficient catalyst for the smooth and selective oxidation of these light alkanes to alcohols will not only provide a solution to the problem of liquifying natural gas, but will create new opportunities to utilize the relatively inexpensive and abundant light alkanes for the production of a variety of valuable fuel and chemical products.

Figure 1-2 shows the relative ease of activation of $\mathrm{C}-\mathrm{H}$ bonds in the kinds of light alkanes that make up natural gas. This trend is reflected in the ease of catalytic air oxidation of the hydrocarbons: isobutane $>$ butane $>$ propane $>$ ethane $>$ methane. The conversion of any major gas component as well as the entire gaseous mixture to an alcohol-rich oxidate has value both to the gas producer and the liquid transportation fuel producer alike. We are currently capable of oxidizing isobutane well and and could be ready for proof-of-concept of a propane to IPA process within two years. Ethane and methane oxidation will require more time than this. Thus it was proposed that the proof-of-concept stage proceed in several discrete stages: isobutane to TBA, propane to IPA, (ethane to ethanol optional), and finally methane to methanol. Concurrent with the developmental aspects of each of these phases will be research phases geared to enable us to produce catalysts active enough for each succeeding phase. 


\subsection{PROGRAM ELEMENTS}

Program elements are reviewed in the first Quarterly Report, (January 1-March 31, 1993), of the Cooperative Agreement. Table 3.1 indicates where Phase IV falls in the original, overall plan for the extended Cooperative Agreement.

Subsequent sections of this Final Report highlight the most successful areas of research during the course of the Cooperative Agreement. Comprehensive coverage of all work done on the project can be found in Topical Reports I-VI. 
TABLE 3.1

COMPONENTS OF NEW COOPERATIVE AGREEMENT

TIME

$2 / 93-2 / 94$

2/94- 2/95

Phase V TBA Development

Phase VI $\mathrm{C}_{1}-\mathrm{C}_{3}$ Research
APPROX. LEVEL OFEFFORT

$\$ 1.0 \mathrm{MM}$

$\$ 2.0 \mathrm{MM}$

$\$ 2.1 \mathrm{MM}$

$\$ 0.9 \mathrm{MM}$

(Research Terminated 10/31/95) 


\subsection{REACTION OF ALKANES WITH DIOXYGEN- TOWARD SUPRABIOTIC SYSTEMS}

\subsection{INTRODUCTION}

Catalytic alkane conversion science and technology is maturing to the point that one can envision economical processes for upgrading the light alkanes currently found in great abundance in natural gas and light refinery streams. Application of this science to the conversion of these light alkanes directly to alcohols using molecular oxygen would make possible the utilization of inexpensive aliphatic hydrocarbons for producing valuable chemical and fuel products.

Until recently, there have been no effective catalysts for the selective efficient conversion of the light alkanes - methane, ethane, propane and the butanes - to alcohols using air or oxygen as the sole oxidant. Processes based on heterogeneous catalysts use air or oxygen but normally are conducted at elevated temperatures and produce deeply oxidized products such as carboxylic acids and anhydrides. Homogeneous catalysts have also proven to be relatively ineffective for the air-oxidation of light alkanes to alcohols and this fact has spawned tremendous interest in biological systems.

Cytochrome P-450 and methane monooxygenase have been found to convert alkanes to alcohols in vitro (1-7). Both heme and non-heme iron centers have been implicated in the oxygen atom transfer step. Growing understanding of the biological systems together with the need for catalysts which can activate aliphatic $\mathrm{C}-\mathrm{H}$ bonds for selective hydroxylation has stimulated intense activity in attempting to mimic biological systems with synthetic analogs (6-14). Much important knowledge has been gained which is highly relevant to the design of catalytic systems for selective air-oxidation of alkanes to alcohols. In addition, this work has brought into focus the problems with the biological systems for operation in an abiotic medium. An acceptable catalyst will be one which effectively applies relevant aspects of the biological systems while circumventing or solving the problems that accompany the applications of enzymic systems.

\subsection{BIOMIMETIC OXIDATION}

\section{A. Cytochrome P-450}

The monoxygenase, cytochrome $\mathrm{P}-450$, contains an iron center in a porphyrinic environment which, in the presence of reduced nicotinamide adenine dinucleotide (NADH), can activate molecular oxygen to form a high-oxidation state iron oxo $(\mathrm{Fe}=\mathrm{O})$ species which is capable of hydroxylating the $\mathrm{C}-\mathrm{H}$ bond of an alkane (1-4). In vitro experiments have shown that hydrocarbons such as norbornane (15), octane $(16,17)$, and hexane (18), can be catalytically hydroxylated using this system. Conditions are mild $\left(20^{\circ} \mathrm{C}, 1 \mathrm{Atm}\right.$. air) and reaction rates are moderately high $(>40 \mathrm{moles}$ alcohol $/ \mathrm{mole}$ enzyme/min.) (19). The function of NADH in this system is to furnish the necessary electrons and protons to the iron center to generate the active species, Eq. 1.

$$
' \mathrm{Fe}^{\prime}+\mathrm{NADH}+\mathrm{H}^{+}+\mathrm{O}_{2}--->\mathrm{Fe}=\mathrm{O}^{\prime}+\mathrm{NAD}^{+}+\mathrm{H}_{2} \mathrm{O}
$$




\section{B. Useful Concepts for Catalysis}

Among the most useful concepts emanating from the many attempts to mimic enzymatic systems is the idea that high oxidation state iron intermediates are the active sites for alkane oxidation (8). In the case of the heme-iron system of cytochrome P-450, an iron oxo intermediate has been implicated (4a). Porphyrinato iron (III) complexes have been used in attempts to model this biocatalyst.

As shown by the dotted line in Figure 1, single oxygen atom transfer reagents, $\mathrm{XO}$, have been used to circumvent the need for two electrons and two protons provided in the natural system by $\mathrm{NADH}$.

Groves (9), in 1979, first used iodosylbenzene as an oxygen atom donor to $\mathrm{Fe}$ in $\mathrm{FeTPPCl}$ and was able to hydroxylate adamantane to adamantanol-1 and adamantanol-2. Groves, (20) using $\mathrm{Cr}$ $\mathrm{TPPCl}$ and iodosylbenzene, was able to isolate an intermediate $\mathrm{Cr}(\mathrm{V})$ complex which was characterized as a $\operatorname{Cr}(\mathrm{V})=0$, chromyl complex.

Many other workers have used a variety of single oxygen donors to act as a "shunt" reagent to form high oxidation state metal-oxo species that are catalytically active. These high oxidation state metal oxo complexes apparently convert aliphatic hydrocarbon substrates to alcohols. It appears, therefore, that if one can generate these species in situ, alkanes will be converted to alcohols. In this paper we will concentrate on metal porphyrin-catalyzed alkane oxidations which use air or oxygen as the oxidant. A recent review of other oxygen sources including a thorough review of single oxygen atom donor work has been published by Meunier (21).

Another useful concept arising from the studies of biological oxidations carried out to date is the utility of a rigid hydrophobic pocket around the iron which attracts the alkane from an aqueous medium and rejects alcohol product to prevent over oxidation. The shape and size of the pocket orients the hydrocarbon molecule for hydroxylation. Its rigidity also helps to prevent self-oxidation which can result in destruction of the complex. Thus a catalyst which could generate high oxidation state iron oxo units in a hydrophobic pocket could exhibit high oxidation activity without ligand destruction and with high selectivity to the alcohol.

\section{C. $\underline{Q}_{2}$-Based Hydroxylations with Sacrificial Reductants}

The major problem with using either biological or biomimetic systems investigated to date for practical catalytic oxidations is the stoichiometric consumption of expensive coreductants or oxygen atom transfer reagents. Biological systems use NADH stoichiometrically in the production of the active iron $(\mathrm{Fe}=\mathrm{O})$ centers. The NAD which is formed in this process must be reduced by some reagent which is sacrificed in order to return the NAD to NADH. In synthetic systems, it has been possible to replace the single atom oxygen donors with dioxygen for metalloporphyrin catalyzed hydroxylation by the use of sacrificial coreductants. The simplest means which has been found to date to furnish the required two electrons and two protons to a biomimetic catalyst systems is to split the 
hydrogen molecule over a group VIII metal catalyst in situ, Figure 2. Tabushi and Yazaki (22) were able to use $\mathrm{MnTPPCl}$ with imidazole and $\mathrm{Pt} / \mathrm{H}_{2}$ to hydroxylate adamantane with air although the rate was very slow. Although it has been successful only for conversion of very reactive hydrocarbons, such an approach could provide the possible pathway shown in Eq. 2 for the direct conversion of alkanes into alcohols. But even this relatively straightforward approach would probably be too costly to convert natural gas components or other inexpensive alkane feedstocks to alcohols for fuel use since it requires stoichiometric consumption of one mole of hydrogen per mole of alkane converted. However, if the catalytic reaction shown in eq. 2 could be accomplished in good yield at a reasonable rate, it could have utility in producing chemicals valuable enough to bear the cost of hydrogen consumption.

$$
\mathrm{RH}+\mathrm{O}_{2}+\mathrm{H}_{2} \stackrel{\text { Cat. }}{\longrightarrow} \mathrm{ROH}+\mathrm{H}_{2} \mathrm{O}
$$

Not only $\mathrm{H}_{2}$ but several other sacrificial chemical reductants have enabled metalloporphyrins to catalytically activate dioxygen towards alkane hydroxylation. Tabushi and Koga (23) were the first to utilize chemical reductants in metalloporphyrin catalyzed oxidations. Using $\mathrm{NaBH}_{4}$ as the reductant together with added imidazole, they found that $\mathrm{MnTPPCl}$ catalyzed predominant epoxidation of cyclohexene. Tabushi (24) postulates that when colloidal $\mathrm{Pt} / \mathrm{H}_{2}$ is used as the reductant, $\mathrm{Mn}$ (III) is reduced to dioxygen-active $\mathrm{Mn}(\mathrm{I})$, eventually leading to a high-valent $\mathrm{Mn}=\mathrm{O}$ active site.

Mansuy et.al., $(25,26)$ developed a hydroxylation system that employs ascorbate ion reduction with $\mathrm{MnTPPCl}, \mathrm{O}_{2}$, and a phase transfer agent to aid the transfer of an electron from the aqueous ascorbate to the organic phase containing the metalloporphyrin. Using this system cyclohexane was converted to a mixture of cyclohexanol and cyclohexanone and heptane to all the heptanones. $\mathrm{FeTPPCl}$ and CoTPP were also used (27) with ascorbate ion to hydroxylate cyclohexane. Other reducing systems which have been studied include ones which use propionaldehyde (28), $\mathrm{Zn},(29)$ $\mathrm{Zn}(\mathrm{Hg})(30)$, and ferrocene (31).

The key concept that this work demonstrates is that reduction to the oxygen active state of the metal [e.g. Fe(II) or $\mathrm{Mn}(\mathrm{II})$ ] is essential for completing a catalytic cycle. Whether a high oxidation state metal-oxo complex is obtained from these systems is unknown but the ability to utilize dioxygen is inextrictably tied to the ability to reach a low 'oxygen-active'oxidation state of the metal.

\section{D. $\underline{Q}_{2}$-Based Hydroxylations Utilizing Electrode Processes}

Electrochemical based catalytic systems provide the electrons which replace the chemical reductants discussed above. For the metalloporphyrin catalyzed hydroxylation of alkanes two types of approaches are viable. One utilizes a soluble metalloporphyrin and an external electrode and in the second the metalloporphyrin is incorporated as part of the electrode itself.

Mansuy, et.al. (32) were able to oxidize alkanes such as cyclooctane, adamantane, and ethylbenzene to mixtures of alcohols and ketones by using $\mathrm{O}_{2}$ in an electrochemical cell consisting of a platinum gauze working electrode with $\mathrm{MnTPPCl}$, imidazole, electrolyte, and acetic acid in 
acetonitrile. The yield based on electrons consumed was low and the rate slow, however, hydroxylation was achieved.

Bedioui, et.al. (33) constructed a graphite electrode coated with a film of a polypyrrole-substituted manganese porphyrin. With this electrode in the presence of 1-methylimidazole, hydrocarbon substrate, supporting electrolyte and bubbling $\mathrm{O}_{2}$, they were able to oxidize tetralin, indane, and cyclooctane. The rates were faster than the soluble system of Mansuy but yield per electron was low and actual productivity was quite poor since only $10^{-8}$ to $10^{-7}$ moles of manganese porphyrin could be attached to the electrodes.

\section{E. $\underline{Q}_{2}$-Based Hydroxylations Utilizing Light}

Irradiation into the ligand to metal change transfer band in $\mathrm{FeTPPCl}$ caused the photoreduction of the iron to the ferrous state but only in the presence of hydrocarbons with weak C-H bonds such as cumene and cyclohexene (34). In the presence of these hydrocarbons and oxygen, photoinitiation of autoxidation occurred giving the usual radical chain oxidation products.

In similar work $\mathrm{Fe}$ (TDCPP)OH was irradiated with $350-450 \mathrm{~nm}$ light producing $\mathrm{Fe}(\mathrm{II})$ and initiating oxidation of cyclohexane by $\mathrm{O}_{2}(35)$.

\subsection{THE SUPRABIOTIC APPROACH}

To mimic simply the biological systems may not be sufficient to generate active stable and cost effective catalysts for alkane oxidations. An abiotic catalyst which employs an active metal oxo intermediate must have several features that are above and beyond the capability of biological systems. An important feature would be to be able to generate the metal oxo species directly from molecular oxygen without the costly requirement of a stoichiometric added coreductant.

A major problem with biomimetic systems is deactivation of the catalyst by oxidative destruction. Two ways in which this comes about are by a) $\mu$-oxo dimer formation and b) oxidative degradation of the ligand system. Nature uses a hydrophobic pocket to deter these deactivation processes. By rigidly holding the iron porphyrin in place the hydrophobic pocket prevents two iron atoms from getting close enough to form inactive species. It also keeps weak C-H bonds away from the active center so that the organic system stays intact. Nature has regeneration mechanisms to combat destruction of portions of the enzyme system - a synthetic abiotic system would not posess such possibilities. Any major destruction of the ligand system in a synthetic catalyst would result in loss of both selectivity and activity. Abiotic catalysts must be inherently robust to thermal and oxidative degradation in order to survive the rigors of industrial processing or laboratory synthesis.

Thus, a suprabiotic catalyst is one that does not require stoichiometric consumption of a reductant $\left(2 \mathrm{e}^{-}+2 \mathrm{H}^{+}\right)$for cleaving $\mathrm{O}_{2}$, uses both atoms of the $\mathrm{O}_{2}$ molecule to form product (exhibits dioxygense activity), and is robust with respect to reaction conditions. In this treatise, we will describe a rationale for creating just such a suprabiotic system. We will provide initial results which indicate that a good measure of success has already been achieved, and we will propose additional 
work for further development of new suprabiotic catalyst systems in order to produce efficient processes for converting light alkanes to valuable fuels and chemicals.

When searching for a possible model catalytic system that could meet the requirements of a suprabiotic catalyst, we formulated the catalytic cycle (36) shown below in Figure 3 . Since nature uses a porphyrinic macrocycle as the ligand system this seemed to be a reasonable starting point for this research. The cycle shown below, Figure 3, uses elementary steps most of which have precedent in inorganic chemistry. In this hypothetical cycle oxygen reacts with the metal center to form a superoxo complex which combines with another center to form a $\mu$-peroxo complex. Cleavage of the $\mathrm{O}-\mathrm{O}$ bond in the peroxo complex leads to the metal oxo complex which oxidizes the alkane. One of the reasons why this doesn't happen readily under normal circumstances is that the highly reactive metal oxo complex combines rapidly with a metal center to form a catalytically inactive $\mu$-oxo complex, Eq. 3.

$$
\mathrm{M}=\mathrm{O}+\mathrm{M}---->\mathrm{M}-\mathrm{O}-\mathrm{M}
$$

One goal of suprabiotic catalyst design, therefore, might be to adjust the electronic characteristics of the ligand system about the metal center in such a way as to encourage $\mathrm{O}-\mathrm{O}$ bond cleavage, and to stabilize monomeric metal species relative to $\mu$-oxo intermediates.

\section{A. Catalyst Design Concept}

As we first envisioned a suprabiotic catalyst, it was one which could operate in the absence of costly stoichiometric co-reductants using only air to oxidize an alkane to an alcohol but in some respects mimic biology in that the active intermediates would be similar. It appears as though the active intermediate which oxidizes alkanes in both Cytochrome P-450 and methane monoxygenase is a ferryl species, $\mathrm{Fe}=\mathrm{O}$. We devised a possible conceptual route to active ferryl using only iron, air and substrate, Figure $3(36)$.

Until recently, no success had been achieved in attempting to oxidize alkanes to alcohols with only air or oxygen in this manner. Iron porphyrins have been tried with $\mathrm{O}_{2}$ and considered as autoxidation catalysts (37) but were able to oxidize only activated benzylic $\mathrm{C}-\mathrm{H}$ bonds such as those in tetralin. Metallophthalocyanines have been used as autoxidation catalysts $(38,39)$ but only recently (38) has work with halogenated phthalocyanines appeared to show reactivity that is superior to classical metal promoted autoxidation.

The requirements for a suprabiotic system for air-oxidations of alkanes to alcohols are many. First, although formation of $\mu$-oxo dimer from the iron(II) precursor is detrimental, $\mu$-peroxo dimer formation is crucial. Secondly, access of the alkane to the active center must be good. Thirdly, the alkyl radical formed by $\mathrm{C}-\mathrm{H}$ bond homolysis should remain in the coordination sphere until rebound to the alcohol occurs. Fourth, the alcohol should be expelled as quickly from the coordination sphere as possible to prevent over-oxidation. Next, $\mu$-oxo dimer formation must be reversed or prevented. Finally, the iron(III)/(II) reduction potential must be high enough to continuously regenerate active $\mathrm{Fe}$ (II) from the pool of $\mathrm{Fe}$ (III) even in the presence of molecular oxygen. In addition, the ligand system must be robust to oxidation, and must not have electrons readily available to reduce the high 
oxidation state ferryl before it can oxidize the alkane. The kinds of porphyrin complexes available in the past did none of these things and consequently were inactive catalysts.

Using perhaloporphyrin complexes it is possible to: a) stabilize active iron-oxygen centers to both of the kinds of oxidative decay referred to above, b) provide a lipophilic environment to attract the alkane to the active site, and thereby provide a hydrophilic environment to expel alcohol from the coordination sphere, c) tune the redox potential of the metal center so that the $\mu$-oxo dimer is no longer inactive and so that iron(II) species may be continually regenerated in an oxidizing environment. Figure 4 suggests how perhaloporphyrins may act as a suprabiotic catalyst for converting alkanes to alcohols. We will discuss how the electronic and steric environment about the oxidation-active metal center affects the rate and selectivity of alkane oxidation in homogeneous liquid phase oxidations. Reaction rate seems to be highly dependent on proper tuning of redox potentials. Unprecedented reaction rates have been achieved in the liquid phase and much higher rates may still be possible.

While steric effects influence rate as well, we are finding that their prime importance is in reaction selectivity. Of importance here is steric inhibition of $\mu$-oxo dimer formation while still allowing the reductive binding of dioxygen between two metalloporphyrin moieties. Ejecting formed alcohol from the coordination sphere is also of great importance as we have seen in both homogeneous and heterogeneous alkane oxidation. The greatest gains to date have been in tuning the redox potential for high activity, and there is still room for significant improvement here. In the future, more attention must be given to those aspects of catalyst structure that will effect selectivity to alcohol. Of all of these structural factors, one of the most important is hydrophobicity of the ligand environment.

It should be noted at this point that we are proposing a rather novel solution to the ' $\mu$-oxo dimer problem'. Most efforts in the past have sought to prevent formation of this unreactive species solely by steric hindrance which prevents the iron centers from getting close to one another $(40,41)$. Our approach is largely an electronic rather than a steric one. An implication of this approach is that even a $\mu$-oxo complex which has been properly tuned electronically might provide a catalytically sufficient standing concentration of active metal oxo.

An important aspect of catalyst design employed in this work is the utilization of a non-oxidizable ligand. Firstly the ligand cannot be oxidized by the very high oxidation states generated at the metal center. For example, if one attempts to form an iron(V) oxo species by treatment of $\mathrm{FeTPPCl}$ with iodosylbenzene, one forms instead the iron (IV) radical cation by electron transfer from the porphyrin ligand (42). The ligands used in this work must be resistant to this kind of electron transfer and provide a stable coordination sphere even for metals in very high oxidation states. It goes without saying that the ligands employed must be inert to autoxidation and other potentially destructive processes.

Finally, the alkane must be brought into the coordination sphere of the metal. Biology uses the strategy of the hydrophobic pocket. It should be realized, however, that biological systems operate in an aqueous medium. The complexes used for liquid phase oxidation in our work are hydrocarbon 
soluble. Although affinity of the substrate for the catalyst is a desirable kinetic factor, this component is not easily built into a catalyst. Future generations of catalysts may address this opportunity.

\subsection{CATALYSIS BY ELECTRON DEFICIENT METALLOPORPHYRINS}

\section{A. Effects of Axial Ligation}

Initial studies (43) used tetraphenylporphyrinato metal(III) complexes of first row transition metals including chromium, manganese and iron as catalysts for the reaction of a series of light alkanes with molecular oxygen, Table 1. This series was comprised of three hydrocarbons having medium to strong carbon-hydrogen bonds: isobutane $(94 \mathrm{kcal} / \mathrm{mole})$, propane $(96 \mathrm{kcal} / \mathrm{mole})$, and ethane $(98$ $\mathrm{kcal} / \mathrm{mole}$ ). Isobutane oxidations were carried out at $80^{\circ} \mathrm{C}$, propane was oxidized at $150^{\circ} \mathrm{C}$, and ethane at $200^{\circ} \mathrm{C}$. When the axial ligand, $\mathrm{X}$, was chloride or acetate, no activity was observed for any of these hydrocarbons under these conditions. When the axial ligands were azide or nitride, Table 1 , isobutane and propane were oxidized at an appreciable rate but ethane was unreactive. The macrocyclic ligand system, however, was not oxidatively stable and was rapidly destroyed during the reaction resulting in catalyst deactivation. The azide effect may be related to the ease of autoreduction of the azidometal(III) complex to give metal(II). The reason for the activity of manganese and chromium nitrido complexes is unclear.

\section{B. Benefits of Subsituting Electron-Withdrawing Groups for $\mathrm{H}$ on Catalyst Life and Activity}

In order to make the macrocycle more oxidatively stable, all of the $\mathrm{C}-\mathrm{H}$ bonds of the phenyl groups in the tetraphenylporphyrinato ligand were replaced with $C-F$ bonds $(44,45)$. This had the effect of reducing the oxidizability of the ligand both by molecular oxygen and by the metal itself. Although modest gains in activity were observed for manganese and chromium porphyrin catalyzed reactions, tetrakispentafluorophenylporphyrinato iron complexes were more active by an order of magnitude than their unfluorinated counterparts, Table 2. In all cases the half life of the catalysts improved as well.

It is possible that the superior results observed for the iron complexes were due to the high activity of an iron oxo intermediate which was stabilized by a ligand with strong electron withdrawing groups which prevented ligand oxidation and concomitant reduction of the iron. In addition, the extraordinary high $\mathrm{Fe}$ (III)/Fe(II) reduction potential that these complexes exhibit allows the generation of oxygen binding sites even under oxidizing conditions.

Chang and Ebina (12) first noted the increase in catalytic oxidation by increasing the electron withdrawal in the metalloporhyrin. $\mathrm{Fe}$ and $\mathrm{Mn}\left(\mathrm{TPPF}_{20}\right) \mathrm{Cl}$ were found to catalyze the PhIO-mediated oxidation of cyclohexane. The authors attributed a "more electrophylic oxene" as the reason for the increase. Nappa and Tolman (46) examined a series of FeTPP complexes and related the Hammett sigma constants for phenyl substituents to the reaction rate. They saw enhanced oxidation activity due to electron withdrawing groups with $\mathrm{Fe}\left(\mathrm{TPPF}_{20}\right)$ complexes giving the highest yield.

Although significant oxidative stabilization of the ligand was achieved in these systems by replacing twenty hydrogens with fluorine atoms, there are still eight more hydrogens attached to the 
pyrrole rings in the porphyrin. These present additional sites of oxidative instability which should be dealt with. One approach has been to replace these eight hydrogens by halogenation. The perhaloporphyrin complexes were more robust and more active than the partially halogenated catalysts, Tables 3 and 4 .

The beneficial effects of further halogenation at the $\beta$ - or pyrrolic positions of tetraphenyl porphyrins have been demonstrated and exploited by several research groups. Traylor and Tsuchiya (47) brominated the -positions of $\mathrm{Zn}(\mathrm{TDCPP})$ ultimately forming $\mathrm{Fe}\left(\mathrm{TDCPP}-\mathrm{Br}_{8}\right) \mathrm{Cl}$ after removal of zinc and insertion of iron. This complex was able to increase the $\mathrm{C}_{6} \mathrm{~F}_{5} \mathrm{IO}$-mediated oxidation rate of norbornane over the unbrominated $\mathrm{Fe}(\mathrm{TDCPP}) \mathrm{Cl}$.

Dolphin and co-workers $(48,49)$ examined chlorinated metalloporphyrins and found that increased chlorination of the ring led to increased rates of cyclohexane oxidation with $\mathrm{C}_{6} \mathrm{~F}_{5} \mathrm{IO}$. They were able to relate increased election withdrawal from ring halogenation to the $\mathrm{Fe}(\mathrm{III}) / \mathrm{Fe}(\mathrm{II})$ reduction potential.

There are some examples in which increased halogenation of the porphyrin ring has not led to increased oxidation rates. Mansuy and coworkers (50) examined a group of electronegative porphyrins and the yields of alkane oxidation by $\mathrm{PhIO}$ followed the degree of halogenation in most cases. An exception to the trend was the observation that $\mathrm{Fe}\left(\mathrm{TPPF}_{20}\right) \mathrm{Cl}$ seemed to give higher yields than $\mathrm{Fe}\left(\mathrm{TPPF}_{20} \beta-\mathrm{Cl}_{8}\right) \mathrm{Cl}$. These workers propose that there is a greater tendency for the active species to behave like $\mathrm{PFe}(\mathrm{IV})-\mathrm{O}^{\infty}$ than $\mathrm{P}^{+} \bullet \mathrm{Fe}(\mathrm{IV})=\mathrm{O}$ with increased electron withdrawal from the porphyrin rings.

Tsuchiya $(51,52)$ and co-workers were able to perfluorinate iron porphyrin with $\mathrm{CoF}_{3}$ in*pyridine leading to $\mathrm{Fe}\left(\mathrm{TPPF}_{28}\right) \mathrm{Cl}$. They were able to oxidize benzene to phenol with $\mathrm{H}_{2} \mathrm{O}_{2}$ but surprisingly were unable to oxidize cyclohexane with $\mathrm{H}_{2} \mathrm{O}_{2}$.

Another approach to achieving catalyst stability is to switch the porphyrinic systems to the similar phthalocyanines. Success has been achieved (38) in preparing perfluorophthalocyanato metal azides which have somewhat lower activity than that of the porphyrins but longer life. Long life is attributable both to the total lack of $\mathrm{C}-\mathrm{H}$ bonds and to the presence of nitrogens in the bridge positions of the macrocycle. These catalysts do not produce alcohols in selectivity as high as do their porphyrin analogs.

\subsection{ELECTRON DEFICIENT METALLOPORPHYRINS AS CATALYSTS FOR

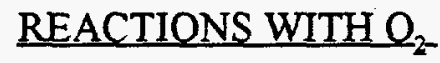

It has been determined $(36,43-45,53)$ that by increasing the amount of electron withdrawal from either the meso- or beta-(pyrrolic) position of the porphyrinato macrocycle surrounding an oxidation active first row metal center, one can greatly increase the $M(I I I) / M(I I)$ reduction potential of the complex. This results in an enormous increase in light alkane oxidation activity and provides us for the first time with a family of metal complexes capable of catalyzing the direct selective air-oxidation of these refractory materials. In studies $(54,55)$ encompassing porphyrin complexes of 
the metals: iron, manganese and chromium, we have shown that the catalytic activity responds positively to electron withdrawal in both the meso= and the beta-positions. It has further been shown that the light alkane air-oxidation activity of the iron complexes of electron deficient porphyrins is superior to that of the other metals.

\section{A. Perhalogenated Metalloporphyrin Catalysts for Combining Alkanes and $\mathrm{O}_{2}$}

Figure 5 shows the porphyrinato macrocycle with the meso- positions labeled $\mathrm{Z}$ and the betapositions labeled $Y$. Our work has shown that while iron complexes of the macrocyclic ligand wherein $\mathrm{Z}=$ phenyl $(\mathrm{Y}=\mathrm{H})$ have very little catalytic activity for air-oxidations of light alkanes at low temperature, those in which $\mathrm{Z}=$ pentafluorophenyl $(\mathrm{Y}=\mathrm{H})$ exhibit high catalytic activity. Changing the $\mathrm{Y}$ group from $\mathrm{H}$ to $\mathrm{X}$, wherein $\mathrm{X}=\mathrm{a}$ halogen such as chlorine or bromine causes further increase in activity. At the time of their discovery, perhalogenated iron complexes were more active than any of the then known metal complexes for light alkane oxidation by at least an order of magnitude and made unprecedented selective low temperature alkane oxidations possible, Table 5. Alcohols are major reaction products and no expensive oxidants or co-reductants are required using these catalysts.

Table 6 compares the isobutane and propane oxidation activity of the series of iron porphyrin complexes described in Figure 5. Although these complexes were the most active known, and perhalogenation had created the most stable porphyrin complexes yet prepared, even more stable and active catalysts are still being generated by further enhancing the electron-withdrawing power of the peripheral substitutents on the porphyrin ring and by adjusting the geometrics of the metal complexes (61).

\section{B. Electron Withdrawal, Reduction Potential, and Catalytic Activity}

In the past, the very critical determinations of $\mathrm{Fe}(\mathrm{III}) / \mathrm{Fe}$ (II) reduction potential had been done in different laboratories and not always under identical conditions. Because of the apparent direct relationship between reduction potential and catalytic activity of iron porphyrin complexes we reinvestigated this area. The $\mathrm{Fe}(\mathrm{III}) / \mathrm{Fe}$ (II) redox potentials vs SCE for the series of iron porphyrins have been determined under a set of consistent conditions. The CV's were all obtained in $\mathrm{CH}_{2} \mathrm{Cl}_{2}$ and tetrabutylammonium chloride was used as supporting electrolyte. A glassy carbon electrode was used. Table 7 lists the half-wave potentials determined for several of the compounds in the series.

As the $\mathrm{C}-\mathrm{H}$ bonds around the periphery of the tetraphenylporphyrinato ligand system are changed to $\mathrm{C}$ - $\mathrm{X}$ bonds $(\mathrm{X}=\mathrm{F}, \mathrm{Cl}, \mathrm{Br})$, the $\mathrm{Fe}(\mathrm{III}) /(\mathrm{II})$ reduction potential increases. Table 7 shows that the catalytically inactive parent iron porphyrin, $\mathrm{Fe}(\mathrm{TPP}) \mathrm{Cl}$, exhibits an $\mathrm{Fe}$ (III)/(II) half-wave potential at $-0.22 \mathrm{~V}$ whereas the $\mathrm{E}_{12}$ exhibited by the highly active perhaloporphyrin complex, $\mathrm{Fe}\left(\mathrm{TPPF}_{20} \mathrm{\beta}-\mathrm{Cl}_{8}\right) \mathrm{Cl}$, is fully $0.5 \mathrm{~V}$ higher. It appears that there is a direct relationship between the extent of electron withdrawal from the porphyrin ring, which causes an increase in the $\mathrm{Fe}$ (III)/(II) reduction potential, and the catalytic activity of the iron complex, Figure 6. 


\section{Porphyrin Ring Buckling, Steric Hindrance, and Catalytic Activity}

What was surprising about Table 7 , however, was that the catalytic activity of $\mathrm{Fe}\left(\mathrm{TPPF}_{20} \beta-\mathrm{Br}{ }_{8}\right) \mathrm{Cl}$ was not lower than that of $\mathrm{Fe}\left(\mathrm{TPPF}_{20} \beta-\mathrm{Cl}_{8}\right) \mathrm{Cl}$. Structural information currently being gathered indicates that the steric bulk of the $\beta$-bromo substituents may prevent access of large molecules to the central iron atom. This has two possible consequences which could affect reaction rate. Firstly, a sterically hindered porphyrin might bind dioxygen to give a $\mu$-peroxo complex which could cleave to form an active ferryl oxo species. It would be unlikely that a highly hindered diron $\mu$-oxo complex, however, would be very stable if it is formed at all. To the extent that ferryl oxo complexes are active intermediates and that diiron $\mu$-oxo species are not, a steric environment which prevents $\mu$-oxo formation could cause an oxidation rate enhancement. Structural features of electron deficient porphyrins also appear to play an important role in catalytic air-oxidation reactions. In particular, steric interactions which could limit or prevent $\mu$-oxo dimer formation may be important for high catalytic activity. We have identified two different types of steric interactions which may be important. One way in which $\mu$-oxo dimer formation could be suppressed is through severe buckling of the porphyrin macrocycle due to steric bulk in the beta-positions. It was seen that as the bulkiness of the $\beta$-substituent increases from $\mathrm{H}$ to $\mathrm{Cl}$ to $\mathrm{Br}$, the extent of buckling increases. It would seem difficult to make the $\mu$-oxo species when the beta-positions bear eight bulky bromines.

Secondly, large electron withdrawing groups such as chlorines in the ortho position of the phenyl rings of tetraphenylporphyrinato complexes make $\mu$-oxo dimer formation difficult at best." The Traylors and Dolphin (41) prepared $\mathrm{Fe}(\mathrm{TDCPP}) \mathrm{Cl}$ and $\mathrm{Fe}\left(\mathrm{TPPCl}_{20}\right) \mathrm{Cl}$ and found them too "sterically hindered" to form $\mu$-oxo dimers and instead form monomeric hydroxide complexes when treated with base.

In order to compare steric effects on catalytic activity, we studied two perhaloporphyrin complexes having similar electrochemical properties but which differ greatly in steric bulk. The complexes: $\mathrm{Fe}\left(\mathrm{TPPCl}_{28}\right) \mathrm{Cl}$ and $\mathrm{Fe}\left(\mathrm{TPPF}_{20} \beta-\mathrm{Cl}_{8}\right) \mathrm{Cl}$, have similar electronic properties $\left[\mathrm{E}{ }_{1 / 2}\right.$ for $\mathrm{Fe}(\mathrm{III}) / \mathrm{Fe}(\mathrm{II})=0.27$ and 0.28 respectively (49)] but access to the iron center in $\mathrm{Fe}\left(\mathrm{TPPCl}_{28}\right) \mathrm{Cl}$ is far more restricted. It is possible that $\mathrm{Fe}\left(\mathrm{TPPF}_{20} \beta-\mathrm{Cl}_{8}\right) \mathrm{Cl}$ could form a $\mu$-oxo dimer. On the other hand, it is not likely that $\mathrm{Fe}\left(\mathrm{TPPCl}_{28}\right) \mathrm{Cl}$ could form a $\mu$-oxo dimer because of the huge non-bonded interactions resulting from the eight ortho-chloro substituents on each macrocycle, which cause significant buckling of the macrocycle.

\section{Oxidation of Alkanes Using Fe(TPPCl $\left.{ }_{28}\right) \mathrm{Cl}$ as Catalyst}

We attempted the reaction of isobutane $(20 \%)$ in benzene with molecular oxygen under standard test conditions (100 psig $\mathrm{O}_{2}, 6$ hours) at 40,60 , and $80^{\circ} \mathrm{C}$ using $\mathrm{Fe}\left(\mathrm{TPPCl}_{28}\right) \mathrm{Cl}$ as the catalyst. No reaction occurred. Under these conditions nearly 2,000 turnovers were observed at $60^{\circ} \mathrm{C}$ using $\mathrm{Fe}\left(\mathrm{TPPF}_{20} \mathrm{\beta}-\mathrm{Cl}_{8}\right) \mathrm{Cl}$ as the catalyst. One interpretation of these results is that not only is the $\mu$-oxo dimer, $\left[\mathrm{Fe}\left(\mathrm{TPPCl}_{28}\right)\right]_{2} \mathrm{O}$, unable to form, but even the $\mu$-peroxo dimer, $\left[\mathrm{Fe}\left(\mathrm{TPPCl}_{28}\right)\right]_{2} \mathrm{O}_{2}$ cannot be made. Inspection of the molecular model of the monomeric Fe(II) complex indicates that the iron 
hindrance about the iron centers is so great that this complex may not be able to reductively bind dioxygen.

Small oxygen atom transfer reagents, however, should still be able to oxidize the iron center to a ferryl intermediate which will oxidize hydrocarbons. It has been found (48) that $\mathrm{Fe}\left(\mathrm{TPPCl}_{28}\right) \mathrm{Cl}$ oxidizes cyclohexane to the alcohol and the ketone and oxidizes cyclohexene to the epoxide when iodosylbenzene is the oxidant.

Thus, when a small oxygen donor molecule having oxygen in the reductively bound state is used, oxidations, presumably occurring through an active ferryl intermediate, are possible. When it is necessary to reductively bind a dioxygen molecule in order to get the active catalytic intermediate, steric bulk may prevent this reaction from occurring.

Therefore, it is possible that we may now have spanned the range of steric requirements for both $\mu$-oxo and $\mu$-peroxo complex formation. It would appear that a properly tuned iron complex which can readily form a $\mu$-peroxo dimer but which cannot form $\mu$-oxo dimer should give the greatest concentration of active ferryl, and thus should be the best catalyst.

\subsection{FUTURE SUPRABIOTIC CATALYSTS}

We have shown that catalysts such as $\mathrm{Fe}\left(\mathrm{TPPF}_{20} \beta-\mathrm{Br}_{8}\right) \mathrm{Cl}$ and $\left.\mathrm{Fe}\left(\mathrm{TPPF}_{20} \beta-\mathrm{Cl}\right)_{8}\right) \mathrm{Cl}$ are superior air oxidation catalysts able to selectively transform alkanes containing tertiary $\mathrm{C}-\mathrm{H}$ bonds such as isobutane and those with secondary $\mathrm{C}-\mathrm{H}$ bonds such as propane to alcohols. Although highly electron deficient porphyrinato complexes could give highly enhanced autoxidation behavior, our work suggests the possibilities of suprabiotic pathways as well. In order to be commercially attractive the rates of these reactions could be faster. In order to attack even less reactive alkanes such as ethane and methane more active catalysts are needed. Can we guess what increasingly active suprabiotic catalysts will look like?

What have we learned at this stage from studies of suprabiotic catalysts? What properties do they possess? We see that several properties are important to the success of a suprabiotic catalyst:

1) electron withdrawal from the metal center via electron withdrawing groups on the periphery of the macrocycle resulting in high $\mathrm{M}(\mathrm{III}) / \mathrm{M}$ (II) reduction potential;

2) removal of reactive $\mathrm{C}-\mathrm{H}$ bonds for increased oxidative stability;

3) sufficient solubility and compatibility with alkane substrate and alcohol product;

4) geometric properties which encourage reductive binding of dioxygen to form binuclear $\mu$-peroxo species; and

5) steric constraints which favor $M=O$ intermediates over binuclear $\mu$-oxo complexes (without preventing reductive binding of $\mathrm{O}_{2}$ ). 


\section{A. Increasing Electron Withdrawal}

Halogenation of groups at the $\beta$ and meso position of metalloporphyrins has been successfully used to increase the electron withdrawal from the metal center leading to superior oxidation catalysts. A tabulation of electron withdrawing capabilities of functional groups such as one of Hammett parameters, Table 8, quickly suggests the use of even more potent electron-withdrawing groups than halogens. Porphyrins having $\mathrm{C}-\mathrm{H}$ bonds replaced with $\mathrm{CF}_{3}$ groups $(56,57), \mathrm{CN}$ groups $(58,59)$, and $\mathrm{NO}_{2}$ groups $(60,61)$ have been prepared

We have shown (62) that $\mathrm{NO}_{2}$ reacts with $\mathrm{Fe}(\mathrm{OEP}) \mathrm{Cl}$ to give $\mathrm{Fe}(\mathrm{OEP})\left(\mathrm{NO}_{2}\right)_{4} \mathrm{Cl}$ and the $\mu$-oxo species $\left[\mathrm{Fe}(\mathrm{OEP})\left(\mathrm{NO}_{2}\right)_{4}\right]_{2} \mathrm{O}$. This $\mu$-oxo species is able to catalyze hydroxylation of isobutane with 5 bars of $\mathrm{O}_{2}$ at $60^{\circ}$ and $80^{\circ}$ giving 800 and 1600 turnovers respectively in five hours in benzene. At room temperature 1000 turnovers were achieved in 48 hours. The selectivety to tert-butyl alcohol is about $90 \%$ in each case. $\mathrm{Fe}(\mathrm{OEP}) \mathrm{Cl}$ or its $\mu$-oxo analogue are inactive even at $80^{\circ}$. Clearly nitration at the four meso positions has overcome the effects of the eight electron-donating ethyl groups. Stimulated by recent results in this field, further examples of suprabiotic catalysts enhanced by powerful electron-withdrawing groups will no doubt soon be inundating the literature.

\section{B. Geometry Control Over Catalysis}

We have seen that the geometry of the porphyrin around the metal center is important to its catalytic power. Inability to form a $\mu$-peroxo dimer could explain the inactivity of a potentially powerful suprabiotic catalyst such as $\mathrm{Fe}\left(\mathrm{TPPCl}_{28}\right) \mathrm{Cl}$.

Control over the porphyrin architecture could provide catalysts which position two irons in proximity for formation of $\mu$-peroxo dimers and homolytic cleavage leading to two $M=0$ centers, Eq.4

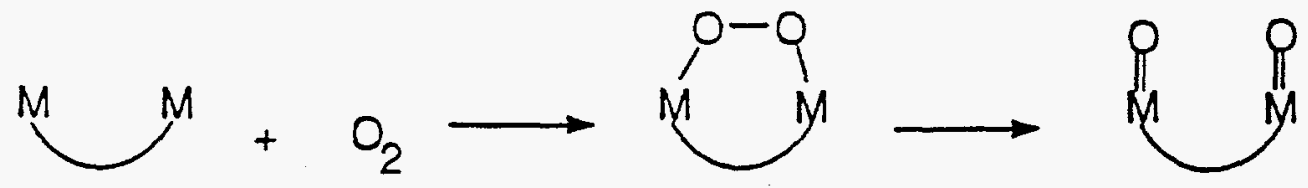

Several workers including Chang (63) and Collman (64) have developed cofacial diporphyrins variations of which may someday be able to perform this task.

Other workers such as Herron, Stucky, and Tolman (65) have isolated metal phthalocyanines in the cages of zeolites to prepare oxidation catalysts (although inactive with $\mathrm{O}_{2}$ ). Inert supports have also been used to disperse metalloporphyrins (66) and metallophthalocyanines (67). This approach seems to be a productive one in instances where the active intermediate is a superoxo species, such as in the Merox process for sulfur oxidation (68). In the case of liquid phase alkane hydroxylation using $\mathrm{O}_{2}$ as the oxidant, it may be necessary to form a $\mu$-peroxo dimer in order to split dioxygen and form the more highly active ferryl species. Since supported mononuclear metalloporphyrins may have difficulty forming $\mu$-peroxo dimers, it might be preferable to either use soluble monomeric 
metalloporphyrins which can react with $\mathrm{O}_{2}$ to form $\mu$-peroxo dimers and ultimately ferryl species or to use proximate metal centers such as those in the aforementioned cofacial diporphyrins.

\subsection{CONCLUSION}

No doubt the development of suprabiotic catalysts will lead to increasingly active perhaps commercially important air/oxygen oxidation catalysts. The increased availability of porphyrin synthetic routes will speed this advance. The need for oxygenated fuels and fuel additives, the possibilities of new applications for suprabiotic catalysts in fine chemical production and even in pollution abatement should make this an important area of research for many years.

\subsection{ABBREVIATIONS}

TPP

TDCPP

$\mathrm{TPPF}_{20}$

$\mathrm{TDCPP} \beta-\mathrm{Br}_{8}$

$\mathrm{TPPF}_{20} \mathrm{\beta}^{-\mathrm{Cl}_{8}}$

$\mathrm{TPPF}_{28}$

$\mathrm{TPPF}_{20} \beta-\mathrm{Br}_{8}$

$\mathrm{TPPCl}_{20}$

$\mathrm{TPPCl}_{28}$

OEP

$\mathrm{OEP}\left(\mathrm{NO}_{2}\right)_{4}$ mesa-tetraphenylporphyrin dianion

mesa-tetrakis(2,6-dichlorophemyl)porphyrin dianion

mesa-tetrakis(pentafluorophenyi)porphyrin dianion

mesa-tetrakis(2,6-dichlorophenyl)-octabromoporphyrin dianion

meso-tetrakis(pentafluorophenyl)-octachloroporphyrin dianion

meso-tetrakis(pentafluorophenyl)-octafluoroporphyrin dianion

meso-tetrakis(pentafluorophenyl)-octabromoporphyrin dianion

meso-tetrakis(pentachlorophenyl)porphyrin dianion

meso-tetrakis(pentachlorophenyl)-octachloroporphyrin dianion

$\beta$-octaethylporphyrin dianion

meso-tetranitro $\beta$-octaethylporphyrin dianion

\subsection{REFERENCES}

1. J. T. Groves, Cytochrome P-450 and other heme-containing oxygenases, Adv. Inorg. Biochem., 1:119 (1979).

2. R. E. White and M. J. Coon, Oxygen activation by cytochrome P-450, Ann. Rev. Biochem., 49: 315 (1980).

3. F. P. Guengerich and T. MacDonald, Chemical mechanisms of catalysis by cytochromes P-450: a unified view: Acc. Chem. Res. 17: 9 (1984).

4a. G. A. Hamilton, Chemical models and mechanisms for oxygenases, Molecular Mechanisms of Oxygen Activation (O. Hayaishi, ed.), Academic Press, New York, p. 405 (1974). 


\subsection{REFERENCES}

(CONTINUED)

4b. P. R. Ortiz de Montellano, Oxygen activation and transfer, Cytochrome P-450 (P.R. Ortiz de Montellano, ed.), Plenum Press, New York, pp. 235-239 (1986).

5. E. J. McKenna and M. J. Coon, Enzymic - Oxidation, IV. Purification and properties of the -hydroxylase of Pseudomonas oleovorans, L. Biol. Chem., 245: 3882 (1970).

6. J. Colby and H. Dalton, Some properties of a soluble methane monooxygenase from Methylococcus capsulatus strain Bath, Biochem. I., 157: 495 (1976).

7. M. P. Woodland and H. J. Dalton, Purification and characterization of component A of the methane monooxygenase from Methylococcus capsulatus (Bath), L Biol.

Chem, 259: 53 (1984).

8. J. T.Groves, T. E. Nemo, and R. S. Myers, Aliphatic hydroxylation via oxygen rebound. Oxygen transfer catalyzed by iron, J. Am. Chem. Soc, 98: 859 (1976).

9. J. T. Groves, T. E. Nemo, and R. S. Myers, Hydroxylation and expoxidation catalyzed by iron-porphine complexes. Oxygen transfer from iodosylbenzene, I Am. Chem. Soc., 101: 1032 (1979).

10. D. Mansuy, J. F. Bartoli, J.C.Chottard, and M. Lange, Metalloporphyrin-catalyzed hydroxylation of cylohexane by alkyl hydroperoxides: pronounced efficiency of iron-porphyrins, Angew. Chem. Int. Ed. Eng., 19: 909 (1980).

11. C. L. Hill and B. C. Schardt, Alkane activation and functionalization under mild conditions by a homogeneous manganese (III) porphyrin-iodosylbenzene oxidizing system, I Am. Chem. Soc. 102: 6374 (1980).

12. C.K. Chang and F. Ebina, NIH shift in haemin-iodosylbenzene-mediated hydroxylations, I. Chem. Soc Chem. Comm. 778 (1981).

13. J. T. Groves and T. E. Nemo, Aliphatic hydroxylation catalyzed by iron porphyrin complexes, L Am. Chem. Soc, 105: 6243 (1983).

14 B. DePorter, M.Ricci, O. Bortolino, and B. Meunier, Catalytic hydroxylation of saturated hydrocarbons with the sodium hypohalite/manganese porphyrin system, I. Mol. Cat., 31 : 221 (1985).

15. J. T. Groves, G. A. McClusky, R. E. White, and M. J. Coon, Aliphatic hydroxylation by highly purified liver microsonal cytochrome P-450. Evidence for a carbon radical intermediate, Biochem. Biophys. Res. Comm., 81: 154 (1978). 


\subsection{REFERENCES}

(CONTINUED)

16. G. Cardini and $P$. Jurtshuk, Cytochrome $P-450$ involvement in the oxidation of $n$-octane by cell-free extracts of Corynebacterium species strain 7E1C, L Biol. Chem.,243: 6070 (1968).

17. G. Cardini and P. Jurtshuk, Enzymic hydroxylation of n-octane by Corynebacterium sp. strain 7E1C, I Biol, Chem.,245: 2789 (1970).

18. K. Morohashi, H. Sadano, Y. Okada, and T. Omura, Position specificity in n-hexane hydroxylation by two forms of cytochrome P-450 in rat liver microsomes, I. Biochem., 93 : 413 (1983).

19. S. G. Sligar and R. I. Murray, cytochrome P-450cam and other bacterial P-450 enzymes, Cvtochrome P-450 (P.R. Ortiz de Montellano, ed.), Plenum Press, New York, p. 478 (1986).

20. J. T. Groves and W. J. Kruper, Jr., Preparation and characterization of an oxoporphinatochromium (V) complex, I Am. Chem. Soc., 101: 7613 (1979).

21. B. Meunier, metalloporphyrins as versatile catalysts for oxidation reactions and oxidative DNA cleavage, Chem. Rev., 92: 1411 (1992).

22. I. Tabushi and A. Yazaki, P-450-type dioxygen activation using $\mathrm{H}_{2}$ /colloidal $\mathrm{Pt}$ as an effective electron donor, I. Am. Chem. Soc., 103: 7371 (1981).

23. I. Tabushi and N. Koga, P-450 type oxygen activation by porphyrin-manganese complex, I. Am. Chem. Soc, 101: 6456 (1979).

24. I. Tabushi, Reductive dioxygen activation by use of artificial P-450 systems. Coord. Chem. Rev., 86: 1 (1988)

25. D. Mansuy, M. Fontecave, and J. F. Bartoli, Mono-oxygenase-like dioxygen activation leading to alkane hydroxylation and olefin epoxidation by an $\mathrm{Mn}(\mathrm{III})$ (porphyrin)-ascorbate biphasic system, I. Chem Soc Chem. Comm., 253 (1983).

26. M. Fontecave and D. Mansuy, Monoxygenase-like oxidation of olefins and alkanes catalyzed by manganese porphyrins:comparison of systems involving either $\mathrm{O}_{2}$ and ascorbate or iodosylbenzene, Tetrahedron, 40: 4297 (1984).

27. L. Ji, M. Liu, A. K. Hsieh, and T. S. A. Hor, Metalloporphyrin-catalyzed hydroxylation of cyclohexane with molecular oxygen, I. Mol. Cat., 70: 247 (1991). 


\subsection{REFERENCES (CONTINUED)}

28. R. Iwanejko, T. Mlodnicka, and J. Poltowicz, Metalloporphyrin-catalyzed oxidation of cyclohexane with dioxygen, New Developments in Selective Oxidation, (G. Centi and F. Trifiro, eds.), Elsevier, Amsterdam, p. 195 (1990).

29. P. Battioni, J. E. Bartoli, P. Leduc, M. Fontecave, and D. Mansuy, A new and efficient biomimetic system for hydrocarbon oxidation by dioxygen using manganese porphyrins, imidazole, and zinc, I Chem. Soc. Chem. Comm., 791 (1987).

30. E. I. Karasevich, A. M. Khenkin, and A. E. Shilov, A chemical model of cytochrome P-450: mono-oxygenase-like activation of dioxygen, L Chem. Soc. Chem. Comm., 731 (1987).

31. G. B. Shulpin and A. N. Druzhinina, Biomimetic activation of the C-H bond. Oxygenation of hydrocarbons with $\mathrm{O}_{2}$ catalyzed by porphyrin complexes of metals in the presence of ferrocene as a reducing agent, Izv Akad. Nauk SSSR, Ser. Khim., 2739 (1991).

32 P. Leduc, P. Battioni, J. F. Bartoli, and D. Mansuy, A biomimetic electrochemical system for the oxidation of hydrocarbons by dioxygen catalyzed by manganese-porphyrins and imidazole, Tett. Lett., 29: 205 (1988).

33. F. Bedioui, S. G. Granados, and J. Devynck, Biomimetic oxidation of hydrocarbons by dioxygen: electrocatalysis using polypyrrole-manganese porphyrin film modified electrodes, New I Chem., 15: 939 (1991).

34. D. N. Hendrickson, M. G. Kinnaird, and K. S. Suslick, Photochemistry of $(5,10,15$, 20-tetraphenylporphyrinato)iron(III) halide complexes, Fe (TPP)(X), J. Am. Chem. Soc., 109: 1243 (1987).

35. A. Maldotti, C. Bartocci, R. Amadelli, E. Polo, P. Battioni, and D. Mansuy, Oxidation of alkanes catalyzed by photoactivated iron porphyrins, I. Chem. Soc. Chem. Comm., 1487 (1991).

36. P. E. Ellis, Jr., and J. E. Lyons, Selective air oxidation of light alkanes catalyzed by activated metalloporphyrins- the search for a suprabiotic system, Coord. Chem. Rev, 105: $181(1990)$.

37. D. R. Paulson, R. Ullman, and R. B. Sloane, Catalysis of autoxidation by metalloporphyrins, I Chem. Soc. Chem. Comm, 186 (1974). 


\subsection{REFERENCES (CONTINUED)}

38. J. E. Lyons and P. E. Ellis, Jr., Azide activation of metallophthalocyanine complexes for the catalyticoxidation of alkanes in the liquid phase, Appl. Cat. A: Gen., 84: L1 (1992).

39. L. Weber, M. Grosche, H. Hennig, and G. Haufe, Oxygenation of alkenes with phthalocyaninato manganese (III) and iron (III) complexes and dioxygen, I. Mol. Cat., 78 : 19 (1993).

40. A. L. Balch, Y. W. Chan, R. J. Cheng, G. N. Lamar, L. Latos-Grazynski, and M. W. Renner, Oxygenation patterns for iron (II) porphyrins. Peroxo and ferryl $\left(\mathrm{Fe}^{\mathrm{IV}} \mathrm{O}\right)$ intermediates detected by ${ }^{1} \mathrm{H}$ nuclear magnetic resonance spectroscopy during the oxygenation of (tetramesitylporphyrin)-iron(II), I. Am. Chem. Soc, 106: 7779 (1984).

41. P. S. Traylor, D. Dolphin, and T. G. Traylor, Sterically protected hemins with electronegative substituents: efficient catalysts for hydroxylation and epoxidation, I. Chem. Soc. Chem. Comm., 279 (1984).

42. A. L. Balch, L. Latos-Grazynski, and M. W. Renner, Oxidation of red ferryl $\left[\left(\mathrm{Fe}^{\mathrm{IV}} \mathrm{O}\right)^{2+}\right]$ porphyrin complexes to green ferryl $\left[\left(\mathrm{Fe}^{\mathrm{IV}} \mathrm{O}\right)^{2+}\right]$ porphyrin radical complexes, $\mathrm{I}$. Am. Chem. Soc., 107: 2983 (1985).

43. P. E. Ellis, Jr., and J. E. Lyons, Effect of axial axide on the selective, low-temperature metalloporphyrin-catalyzed reactions of isobutane with molecular oxygen, J. Chem. Soc. Comm., 1187 (1989)

44. P. E. Ellis, Jr., and J. E. Lyons, Effect of fluorination of the meso -phenyl groups on selective tetraphenylporphyrinatometal (II)- catalysed reactions of isobutane with molecular oxygen, I Chem. Soc. Chem. Comm., 1189 (1989).

45. P. E. Ellis, Jr., and J. E. Lyons, Effect of fluorination of the meso-phenyl groups on selective tetraphenylporphyrinatoiron (II)- catalysed reactions of propane with molecular oxygen, $\mathrm{L}$ Chem. Soc. Chem. Comm., 1315 (1989)

46. M. J. Nappa and C. A.Tolman, Steric and electronic control of iron porphyrin catalyzed hydrocarbon oxidations, Inorg. Chem., 24: 4711 (1985).

47. T. G. Traylor and S. Tsuchiya, Perhalogenated tetraphenylhemins: stable catalysts of high turnover catalytic hydroxylations, Inorg. Chem., 26: 1338 (1987). 


\subsection{REFERENCES (CONTINUED)}

48. D. H. Dolphin, T. Nakano, T. K. Kirk, T. E. Maione, R. L. Farrell, and T. P. Wijeseker Porphyrins, their syntheses and uses thereof, World Int. Patent WO88/07988, October 20 (1988).

49. T. Wijesekera, A. Matsumoto, D. Dolphin, and D. Lexa, Perchlorinated and highly chlorinated meso-tetraphenylporphyrins, Angew Chem. Int. Ed. Engl., 29: 1028 (1990).

50. J. F. Bartoli, O. Brigaud, P. Battioni, and D. Mansuy, Hydroxylation of linear alkanes catalyzed by iron porphyrins: particular efficacy and regioselectivity of perhalogenated porphyrins, I Chem. Soc. Chem. Comm. 440 (1991).

51. S. Tsuchiya, M. Seno, M. Kawai, Fluorine-Substituted Porphyrin Complexes, Ipn. Kokai 90-250883, Oct. 8 (1990).

52. S. Tsuchiya and $M$. Seno, Novel synthetic method of phenol from benzene catalysed by perfluorinated hemin, Chem. Lett., 263 (1989).

53. P. E. Ellis, Jr., and J. E. Lyons, Halogen substituent effects on the catalytic activity of iron porphyrin complexes for selective air-oxidation of alkanes in the liquid phase, Cat. Lett., 3:389 (1989).

54. J. E. Lyons and P. E. Ellis, Jr., Selective low-temperature hydroxylation of isobutane by molecular oxygen catalyzed by an iron perhaloporphyrin complex, Cat. Lett, $\underline{8}: 45$ (1991).

55. J. E. Lyons, P. E. Ellis, Jr., and V. A. Durante, Active iron oxo centers for the selective catalytic oxidation of alkanes, Structure-Activity Relationships in Heterogeneous Catalysis (R. E. Grasselli and A. W. Sleight, eds.), Elsevier, Amsterdam, pp. 99-116 (1991).

56. N. Ono, H. Kawamura, and K. Maruyama, A convenient synthesis of trifluoromethylated pyrroles and porphyrins, Bull. Chem. Soc. Ipn., 62: 3386 (1989).

57. P. E. Ellis, Jr., and J. E. Lyons, Chromium halogenated coordination complexes for the oxidation of butane to methylethylketone, U.S. Pat. 4,970,348, Nov. 13 (1990).

58. H. J. Callot, A. Giraudeau, and M. Gross, Cyanoporphyrins. Co-ordinating and electrochemical properties, J. Chem. Soc. Perkin Trans. 2, 1321 (1975).

59. P. E. Ellis, Jr., and J. E. Lyons, Cyano- and polycyanometalloporphyrins as catalysts for alkane oxidation, U.S. Pat. 5,118,886, June 2 (1992). 


\subsection{REFERENCES (CONTINUED)}

60. E. Watanabe, S. Nishimura, H. Ogoshi, and Z. Yoshida, Orientation of electrophilic mesa-substitution in metallooctaethylporphyrins, Tetrahedron, 31: 1385 (1975).

61. P. E. Ellis, Jr., and J. E. Lyons, Nitrated metalloporphyrins as catalysts for alkane oxidation, U.S. Pat. 5,120,882, June 9 (1992).

62. P. E. Ellis, Jr., and J. E. Lyons, Nitrated metallooctaethylporphines as catalysts for the oxidation of alkanes with molecular oxygen, Cat. Lett., in press.

63. C. K. Chang, M. S. Kuo, and C. B. Wang, Stacked double-macrocyclic ligands II. Synthesis of cofacial diporphyrins, L Heterocyclic Chem., 14: 943 (1977).

64. J. P. Collman, J. E. Hutchinson, M. A. Lopez, A. Tabard, R. Guilard, W. K. Seok, J. A. Ibers, and M. LHer, Synthesis and characterization of a superoxo complex of the dicobalt cofacial diporphyrin $\left[\left(\mu-\mathrm{O}_{2}\right) \mathrm{Co}_{2}(\mathrm{DPB})(1,5 \text {-diphenylimidazole })_{2}\right]\left[\mathrm{PF}_{6}\right]$, the structure of the parent dicobalt diporphyrin $\mathrm{Co}_{2}(\mathrm{DPB})$, and a new synthesis of the free-base cofacial diporphyrin $\mathrm{H}_{4}(\mathrm{DPB})$, I. Am. Chem. Soc., 114: 9869 (1992).

65. N. Herron, G.D. Stucky, and C. A. Tolman, Shape selectivity in hydrocarbon oxidations using zeolite encapsulated iron phthalocyanine catalysts, I. Chem. Soc. Chem. Comm. 1521 (1986).

66. H. Kameyama, H. Suzuki, and A. Amano, Intercalation of Co(II) meso-tetrakis (1-methyl-4-pyridyl) porphyrin into montmorillonite, Chem. Lett., 1117 (1988).

67. M. Chibwe and T. J. Pinnavaia, Stabilization of a cobalt(II) phthalocyanine oxidation catalyst by intercalation in a layered double hydroxide host, I. Chem. Soc. Chem. Comm., 278 (1993).

68. H. Fischer, G. Schultz-Ekloff, T. Buck, D. Wohrle, M. Vassileva, and A. Andreev, Mercaptan adsorption capacity and catalytic oxidation activity of silica-supported phthalocyanines, Langmuir $\underline{8}: 2720$ (1992). 


\section{TABLE 1}

\section{OXIDATION OF ISOBUTANE CATALYZED BY METALLOPORPHYRIN AZIDE AND NITRIDE COMPLEXES}

\begin{tabular}{|c|c|c|c|c|c|c|}
\hline Catalyst & $\begin{array}{l}\text { Catalyst } \\
\text { mmol }\end{array}$ & $\begin{array}{l}\mathrm{O}_{2} \text { Uptake } \\
\text { mmol }^{\mathrm{b}}\end{array}$ & $\begin{array}{l}\text { Catalyst } \\
\text { turnovers }\end{array}$ & \multicolumn{2}{|c|}{$\frac{\text { Products/mmol }}{\mathrm{Me}_{2} \mathrm{CO} \text { t-BuOH}}$} & $\begin{array}{l}\text { Selectivityd } \\
\text { to t-BuOH ? }\end{array}$ \\
\hline $\mathrm{Fe}(\mathrm{TPP}) \mathrm{Cl}$ & 0.025 & 0 & 0 & 0 & 0 & - \\
\hline $\mathrm{Mn}(\mathrm{TPP}) \mathrm{Cl}$ & 0.025 & 0 & 0 & 0 & 0 & - \\
\hline $\mathrm{Mn}(\mathrm{TPP}) \mathrm{Cl}$ & 0.050 & 0 & 0 & 0 & 0 & - \\
\hline $\mathrm{Cr}(\mathrm{TPP}) \mathrm{Cl}$ & 0.025 & 0 & 0 & 0 & 0 & - \\
\hline $\mathrm{Fe}(\mathrm{TPP}) \mathrm{N}_{3}$ & 0.013 & 1.7 & 130 & 0.2 & 2.2 & 92 \\
\hline $\mathrm{Mn}(\mathrm{TPP}) \mathrm{N}_{3}$ & 0.013 & 2.3 & 177 & 0.4 & 3.0 & 88 \\
\hline $\mathrm{Mn}(\mathrm{TPP}) \mathrm{N}_{3}$ & 0.025 & 5.5 & 220 & 0.7 & 6.2 & 90 \\
\hline $\mathrm{Cr}(\mathrm{TPP}) \mathrm{N}_{3}{ }^{2}$ & 0.013 & 3.4 & 262 & 0.4 & 3.3 & 89 \\
\hline $\mathrm{Cr}(\mathrm{TPP}) \mathrm{N}_{3}$ & 0.025 & 6.6 & 264 & 0.8 & 6.3 & 89 \\
\hline$[\mathrm{Fe}(\mathrm{TPP})]_{2} \mathrm{~N}$ & 0.013 & 0 & 0 & 0 & 0 & - \\
\hline $\mathrm{Mn}(\mathrm{TPP}) \mathrm{N}$ & 0.025 & 0 & 0 & 0 & 0 & - \\
\hline $\mathrm{Cr}(\mathrm{TPP}) \mathrm{N}$ & 0.013 & 0 & 0 & 0 & 0 & - \\
\hline \multicolumn{7}{|c|}{ 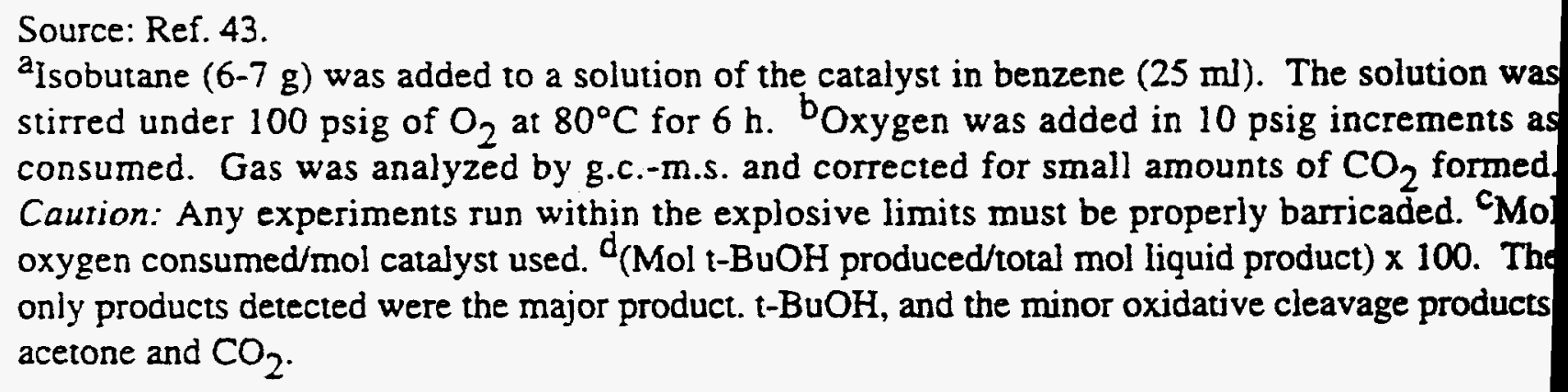 } \\
\hline
\end{tabular}




\section{TABLE 2}

OXIDATION OF ISOBUTANE CATALYZED BY FLUORINATED METALLOPORPHYRIN COMPLEXES

\begin{tabular}{|c|c|c|c|}
\hline Complex & $\begin{array}{l}\text { Amount used } \\
\text { mmol }\end{array}$ & $\begin{array}{l}\text { Catalyst } \\
\text { turnovers } \\
\text { b }\end{array}$ & $\begin{array}{c}\text { Selectivity to } \\
\text { t-BuOH }\end{array}$ \\
\hline $\mathrm{Fe}(\mathrm{TPP}) \mathrm{Cl}$ & 0.025 & 0 & - \\
\hline $\mathrm{Fe}\left(\mathrm{TPPF}_{20}\right) \mathrm{Cl}$ & 0.016 & 2040 & 90 \\
\hline $\mathrm{Fe}(\mathrm{TPP}) \mathrm{N}_{3}$ & 0.013 & 130 & 93 \\
\hline $\mathrm{Fe}\left(\mathrm{TPPF}_{20}\right) \mathrm{N}_{3}$ & 0.016 & 2060 & 89 \\
\hline $\mathrm{Mn}(\mathrm{TPP}) \mathrm{N}_{3}$ & 0.013 & 180 & 88 \\
\hline $\mathrm{Mn}\left(\mathrm{TPPF}_{20}\right) \mathrm{N}_{3}$ & 0.016 & 750 & 87 \\
\hline $\mathrm{Cr}(\mathrm{TPP}) \mathrm{N}_{3}$ & 0.025 & 280 & 89 \\
\hline $\mathrm{Cr}\left(\mathrm{TPPF}_{20}\right) \mathrm{N}_{3}$ & 0.016 & 450 & 97 \\
\hline$[\mathrm{Fe}(\mathrm{TPP})]_{2} \mathrm{O}$ & 0.013 & 0 & - \\
\hline$\left[\mathrm{Fe}\left(\mathrm{TPPF}_{20}\right)\right]_{2} \mathrm{O}$ & 0.007 & $1730^{d}$ & 92 \\
\hline
\end{tabular}

Source: Ref:44.

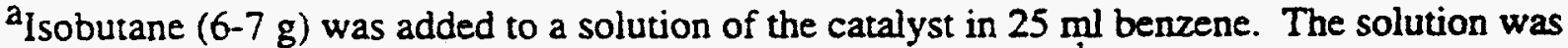
stirred under $\mathrm{O}_{2}(100 \mathrm{psig})$ at $80^{\circ} \mathrm{C}$ for $6 \mathrm{~h}\left(\mathrm{O}_{2}\right.$ added as used). ${ }^{\mathrm{b}}$ Moles oxygen consumed/mole catalyst used. ${ }^{2}$ (Moles $\mathrm{t}-\mathrm{BuOH}$ produced/total moles liquid product) $\times 100$. The only products

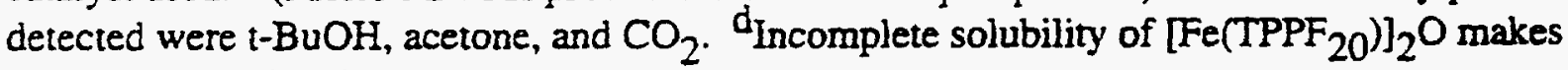
this a lower limit value. 


\begin{tabular}{|c|c|c|c|}
\hline & $\underline{T},{ }^{\circ} \mathrm{C}$ & T.O. b & $\begin{array}{l}\text {-BuOH } \\
\text { Sel..\% } \\
\end{array}$ \\
\hline $\mathrm{Fe}\left(\mathrm{TPPF}_{20} \mathrm{\beta}-\mathrm{Br}_{8}\right) \mathrm{Cl}$ & $\begin{array}{l}60 \\
40 \\
27\end{array}$ & $\begin{array}{c}1890 \\
690 \\
130\end{array}$ & $\begin{array}{l}91 \\
90 \\
90\end{array}$ \\
\hline $\mathrm{Fe}\left(\mathrm{TPPF}_{20} \mathrm{\beta}-\mathrm{Br}_{8}\right) \mathrm{N}_{3}$ & $\begin{array}{l}60 \\
40 \\
27\end{array}$ & $\begin{array}{c}1550 \\
670 \\
620\end{array}$ & $\begin{array}{l}87 \\
89 \\
\text { na }\end{array}$ \\
\hline $\mathrm{Fe}\left(\mathrm{TPPF}_{20} \mathrm{\beta}-\mathrm{Cl}_{8}\right) \mathrm{Cl}$ & $\begin{array}{l}60 \\
40 \\
27\end{array}$ & $\begin{array}{c}1800 \\
760 \\
220\end{array}$ & $\begin{array}{l}90 \\
90 \\
91\end{array}$ \\
\hline $\mathrm{Fe}\left(\mathrm{TPPF}_{20} \mathrm{\beta}-\mathrm{Cl}_{8}\right) \mathrm{N}_{3}$ & $\begin{array}{l}60 \\
40\end{array}$ & $\begin{array}{c}1800 \\
760\end{array}$ & $\begin{array}{l}90 \\
92\end{array}$ \\
\hline $\begin{array}{l}\mathrm{Cr}\left(\mathrm{TPPF}_{20} \mathrm{\beta}-\mathrm{Br}_{8}\right) \mathrm{Cl} \\
\mathrm{Cr}\left(\mathrm{TPPF}_{20} \mathrm{\beta}-\mathrm{Cl}_{8}\right) \mathrm{Cl} \\
\mathrm{Cr}\left(\mathrm{TPPF}_{20} \mathrm{\beta}-\mathrm{Br}_{8}\right) \mathrm{N}_{3} \\
\mathrm{Cr}\left(\mathrm{TPPF}_{20} \mathrm{\beta}-\mathrm{Cl}_{8}\right) \mathrm{N}_{3}\end{array}$ & $\begin{array}{l}60 \\
60 \\
60 \\
80\end{array}$ & $\begin{array}{c}0 \\
70 \\
110 \\
450\end{array}$ & $\begin{array}{l}- \\
\text { na } \\
92 \\
88\end{array}$ \\
\hline $\begin{array}{l}\mathrm{Mn}\left(\mathrm{TPPF}_{20} \mathrm{\beta}-\mathrm{Br}_{8}\right) \mathrm{Cl} \\
\mathrm{Mn}\left(\mathrm{TPPF}_{20} \mathrm{\beta}-\mathrm{Cl}_{8}\right) \mathrm{Cl} \\
\mathrm{Mn}\left(\mathrm{TPPF}_{20} \mathrm{\beta}-\mathrm{Br}_{8}\right) \mathrm{N}_{3} \\
\mathrm{Mn}\left(\mathrm{TPPF}_{20}{ }^{\mathrm{B}-\mathrm{Cl}}{ }_{8}\right) \mathrm{Cl}+\mathrm{NaN}_{3} \mathrm{C} \\
\mathrm{Mn}\left(\mathrm{TPPF}_{20} \mathrm{\beta}-\mathrm{Cl}_{8}\right) \mathrm{Cl}+\mathrm{NaN}_{3} \mathrm{C}\end{array}$ & $\begin{array}{l}60 \\
60 \\
60 \\
80 \\
60\end{array}$ & $\begin{array}{c}110 \\
0 \\
270 \\
1289 \\
156\end{array}$ & $\begin{array}{l}87 \\
- \\
87 \\
86 \\
88\end{array}$ \\
\hline $\mathrm{Co}\left(\mathrm{TPPF}_{20} \mathrm{\beta}-\mathrm{Cl}_{8}\right)$ & 60 & 355 & 91 \\
\hline
\end{tabular}

a Isobutane $(7 \mathrm{~g})$ was oxidized $(6 \mathrm{hrs}$.) in $25 \mathrm{ml}$ benzene at the designated temperature under 100 psig oxygen using 0.013 mmole of the designated catalyst.

b Moles of oxygen consumed/mole catalyst used.

c After warming the reaction mixture for $6 \mathrm{hr}$. as indicated in footnote ${ }^{\mathrm{a}}$, the reaction mixture was cooled to room temperature, $10 \mathrm{mg}$ of $\mathrm{NaN}_{3}$ added and the procedure repeated according to footnote. The T.O. are calculated based on the second $6 \mathrm{hr}$. period. 
PROPANE OXIDATIONS USING FIRST ROW METAL COMPLEXES OF TPPF $_{20} \mathrm{~B}_{-B_{8}}$ AS CATALYSTS $^{\mathrm{a}}$

Time .

\begin{tabular}{|c|c|c|c|c|}
\hline & $\begin{array}{l}\text { Time. } \\
\text { Hours }\end{array}$ & $\underline{T},{ }^{\circ} \mathrm{C}$ & T.O. b & $\begin{array}{c}\text { IPA } \\
\text { Acetone }^{c}\end{array}$ \\
\hline \multirow{2}{*}{$\mathrm{Fe}\left(\mathrm{TPPF}_{20}\right) \mathrm{N}_{3}$} & 3 & 125 & 330 & 0.8 \\
\hline & 6 & 125 & 410 & 0.7 \\
\hline $\mathrm{Fe}\left(\mathrm{TPPF}_{20} \mathrm{P}^{\mathrm{\beta}-\mathrm{Br}} \mathrm{r}_{8}\right) \mathrm{Cl}$ & 4.5 & 125 & 420 & 1.0 \\
\hline $\mathrm{Fe}\left(\mathrm{TPPF}_{20} \mathrm{~B}^{\left.\mathrm{B}-\mathrm{Br}_{8}\right) \mathrm{Br}}\right.$ & 4.5 & 125 & 470 & 0.8 \\
\hline $\mathrm{Fe}\left(\mathrm{TPPF}_{20} \mathrm{~B}-\mathrm{Br}_{8}\right) \mathrm{N}_{3}$ & 4.5 & 125 & 514 & 0.9 \\
\hline $\mathrm{Cr}\left(\mathrm{TPPF}_{20}\right) \mathrm{N}_{3}$ & 4.5 & 125 & 41 & $<0.1$ \\
\hline $\mathrm{Cr}\left(\mathrm{TPPF}_{20} \mathrm{\beta}-\mathrm{Br}_{8}\right) \mathrm{Cl}$ & 4.5 & 125 & 111 & 0.3 \\
\hline 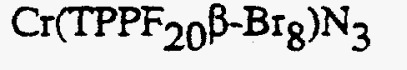 & 4.5 & 125 & 87 & 0.6 \\
\hline $\mathrm{Mn}\left(\mathrm{TPPF}_{20}\right) \mathrm{N}_{3}$ & 3 & 125 & 0 & - \\
\hline $\mathrm{Mn}\left(\mathrm{TPPF}_{20} \mathrm{\beta}-\mathrm{Br}_{8}\right) \mathrm{Cl}$ & 4.5 & 125 & 0 & - \\
\hline $\mathrm{Mn}\left(\mathrm{TPPF}_{20} \mathrm{\beta -Br_{8 }}\right) \mathrm{N}_{3}$ & 4.5 & 125 & 87 & 1.0 \\
\hline $\mathrm{Mn}\left(\mathrm{TPPF}_{20} \mathrm{~B}-\mathrm{Br}_{8}\right) \mathrm{F}$ & 4.5 & 125 & 370 & 0.9 \\
\hline
\end{tabular}

a Propane ( $1.36 \mathrm{~mol})$ was added to benzene $(48 \mathrm{ml})$ containing the catalyst $(0.013 \mathrm{mmol})$. The solution was stirred for 3 hours at $125^{\circ} \mathrm{C}$ under $1000 \mathrm{psig}$ of air in a glass lined autoclave. Liquids and gases were analyzed by GC. Production of carbon oxides never amounted to $10 \%$ of total products. Isopropyl alcohol and acetone exceeded 90 mole \% of carbon containing reaction products in all cases.

b Moles of acetone plus isopropyl alcohol formed per mole of catalyst used.

c Molar ratio isopropyl alcohol to acetone formed. 
TRALE 5

IRON HALOPORPHYRIN CATALYZED I GOBUTANE OXIDATIONS

\begin{tabular}{|c|c|c|c|c|c|c|c|c|c|c|c|}
\hline \multirow[b]{2}{*}{ Catalyet } & \multirow[b]{2}{*}{$\underline{\mathbf{T}^{\circ} \mathrm{C}}$} & \multirow[b]{2}{*}{$\underline{t}$ Hre } & \multirow[b]{2}{*}{$1-C_{4} E_{10}($ mmols } & \multirow[b]{2}{*}{$10_{2}(\mathrm{pg})$} & \multicolumn{4}{|c|}{ Roaction Producte, (mmoles) } & \multirow{2}{*}{$\begin{array}{l}\text { Converaton } \\
{\underline{1-C_{4}}}_{10}{ }_{1}(\%)\end{array}$} & \multirow{2}{*}{$\begin{array}{r}\text { Sol. } * \\
\text { t-Buon }\end{array}$} & \multirow[b]{2}{*}{ TON $^{C}$} \\
\hline & & & & & t-BuOH & Acetono & CO & $\underline{\mathrm{CO}}_{2}$ & & & \\
\hline \multirow[t]{2}{*}{$\mathrm{Fe}\left(\mathrm{TPPF}_{20}\right) \mathrm{OH}$} & 80 & 3 & 1869 & 53 & 310 & 46 & 7 & 18 & 17 & 87 & 11,330 \\
\hline & 24 & 143 & 1871 & 53 & 382 & 17 & $\mathbf{0}$ & 18 & 19 & 95 & 11,600 \\
\hline \multirow[t]{2}{*}{$\mathrm{Fe}\left(\mathrm{TPPF}_{20} \beta-\mathrm{Cl}_{8}\right) \mathrm{Cl}$} & 80 & 3 & 1865 & 142 & 334 & 38 & 6 & 29 & 20 & 88 & 12,400 \\
\hline & 21 & 120 & 1865 & 63 & 366 & 16 & tr & na & 20.5 & 95 & 12,730 \\
\hline \multirow[t]{3}{*}{$\mathrm{Fe}\left(\mathrm{TPPF}_{20} \beta-\mathrm{Br}_{8}\right) \mathrm{Cl}$} & 80 & 3 & 1862 & 148 & 414 & 81 & 6 & 28 & 27 & 84 & 16,500 \\
\hline & 80 & 3 & 1870 & 53 & 277 & 43 & 8 & 23 & 17 & 87 & 10.060 \\
\hline & 24 & 71 & 1862 & 53 & 372 & 35 & tr & 27 & 22 & 92 & 12,150 \\
\hline
\end{tabular}

a Isobutane was oxidized by an oxygen-containing gas mixture $\left(75 \mathrm{~atm}\right.$. diluent $\left.-\mathrm{N}_{2}\right)$ in the liquid phase $(180 \mathrm{ml})$ for 3 hours. Oxygen added as consumed.

b (moles $t$-BuoH/moles liquid product) $\times 100$.

c Moles (t-BuoH and acetone) produced/mole catalyst used. 


\section{TABLE 6}

ALKANE OXIDATIONS CATALYZED BY IRON PORPHYRINS, Fe(TPPZ $\left.20 \beta-Y_{8}\right) x^{a, b}$

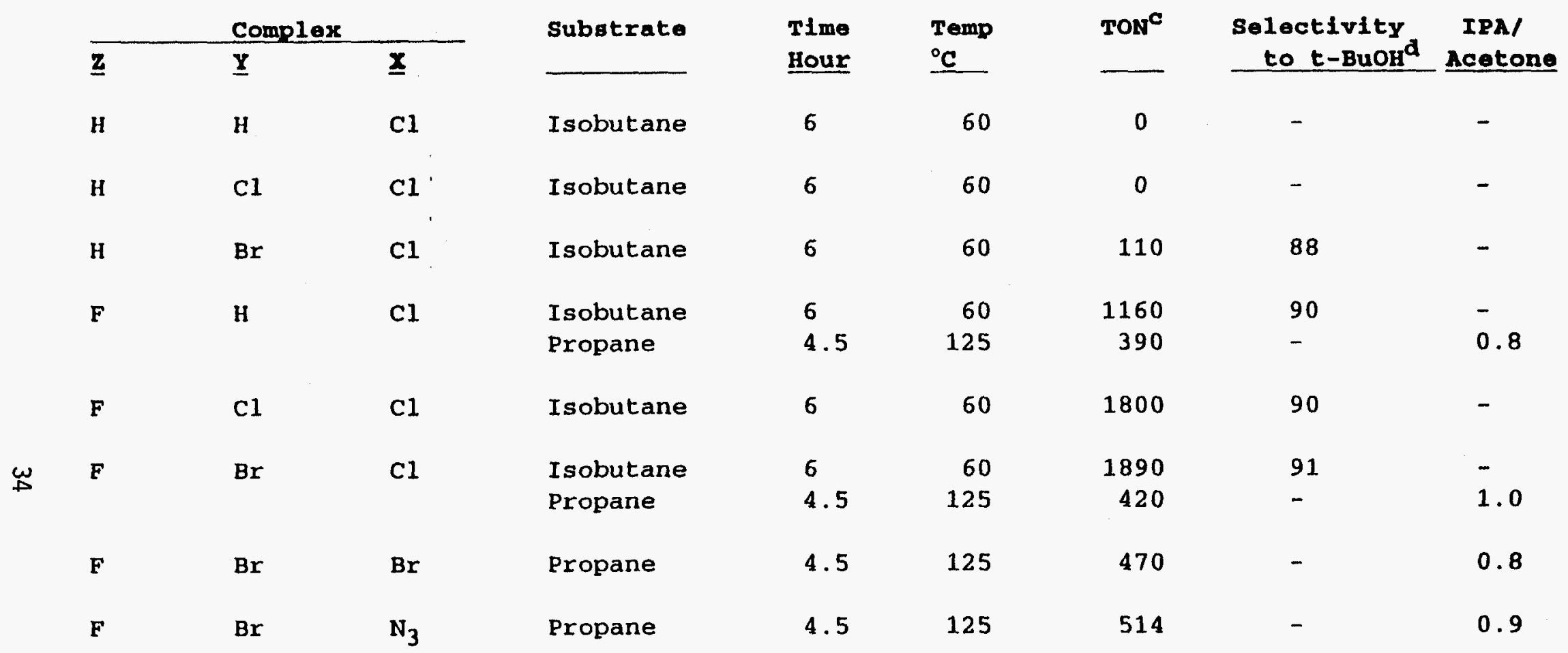

a A solution of 0.013 mmoles of the catalyst in $25 \mathrm{ml}$ benzene containing 6 grams of isobutane was stirred at the designated temperature under $100 \mathrm{psig}$ of $\mathrm{O}_{2}$ for the designated time.

b Stirred solution of 0.023 mmoles of the catalyst in 60 grams of propane in $48 \mathrm{~m} 1$ benzene at $125^{\circ} \mathrm{C}$ under $1000 \mathrm{psig}$ air.

c Moles tert-buty1 alcohol produced/mole catalyst; or Moles (isopropyl alcohol+acetone) formed/mole catalyst used.

d (Moles tert-butyl alcohol produced/total moles liquid product) $\mathrm{x} 100$. 


\section{TABLE 7}

\section{RELATIONSHIP BETWEEN CATALYST REDUCTION POTENTIAL AND ACTIVITY}

\begin{tabular}{|c|c|c|}
\hline CATALYST & $\begin{array}{c}\mathrm{Fe}(\mathrm{III}) /(\mathrm{I}) \\
\mathrm{E} 1 / 2\left(\mathrm{~V}^{\mathrm{a}}\right. \\
\end{array}$ & T.O. \\
\hline $\mathrm{Fe}(\mathrm{TPP}) \mathrm{Cl}$ & -0.221 & 0 \\
\hline $\mathrm{Fe}\left(\mathrm{TPPF}_{20}\right) \mathrm{Cl}$ & +0.07 & 1160 \\
\hline $\mathrm{Fe}\left(\mathrm{TPPF}_{20} \mathrm{~B}-\mathrm{Br}{ }_{8}\right) \mathrm{Cl}$ & +0.19 & 1890 \\
\hline $\mathrm{Fe}\left(\mathrm{TPPF}_{20} \mathrm{\beta}-\mathrm{Cl}_{8}\right) \mathrm{Cl}$ & +0.28 & 800 \\
\hline
\end{tabular}

a Cyclic voltammetry in $\mathrm{CH}_{2} \mathrm{Cl}_{2}$, vs. SCE. tetrabutylammonium chloride supporting electrolyte, glassy carbon electrode.

b Catalyst tumovers for $60^{\circ} \mathrm{C}$ isobutane oxidations in benzene. 
TABLE 8

\section{6-VALUES FOR COMMON FUNCTIONAL GROUPS ${ }^{*}$}

\begin{tabular}{ll} 
GROUP & $\sigma p$ \\
\hline OMe & -0.28 \\
$\mathrm{Me}$ & -0.14 \\
$\mathrm{H}$ & 0 \\
$\mathrm{Ph}$ & 0.05 \\
$\mathrm{~F}$ & 0.15 \\
$\mathrm{Cl}$ & 0.24 \\
$\mathrm{Br}$ & 0.26 \\
$\mathrm{I}$ & 0.28 \\
$\mathrm{CF}_{3}$ & 0.53 \\
$\mathrm{CN}$ & 0.70 \\
$\mathrm{NO}_{2}$ & 0.81
\end{tabular}

* Source: $\sigma_{\mathrm{p}}$ values excerpted from J. March, Advanced Organic Chemistry, 4th Ed., John Wiley and Sons, New York, 1992, p. 280. 
Figure 1 Biological and Biomimetic Alkane Oxidation
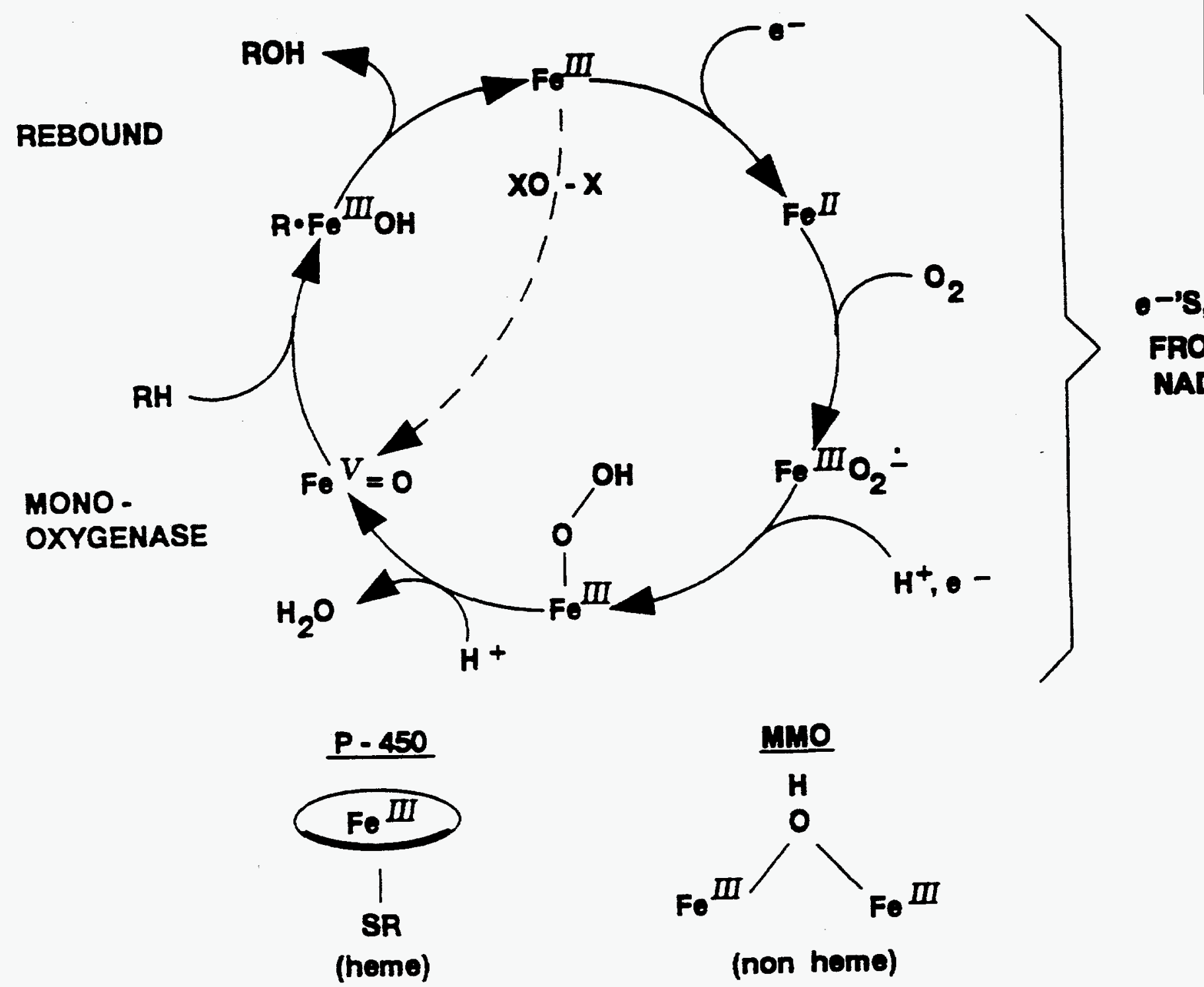


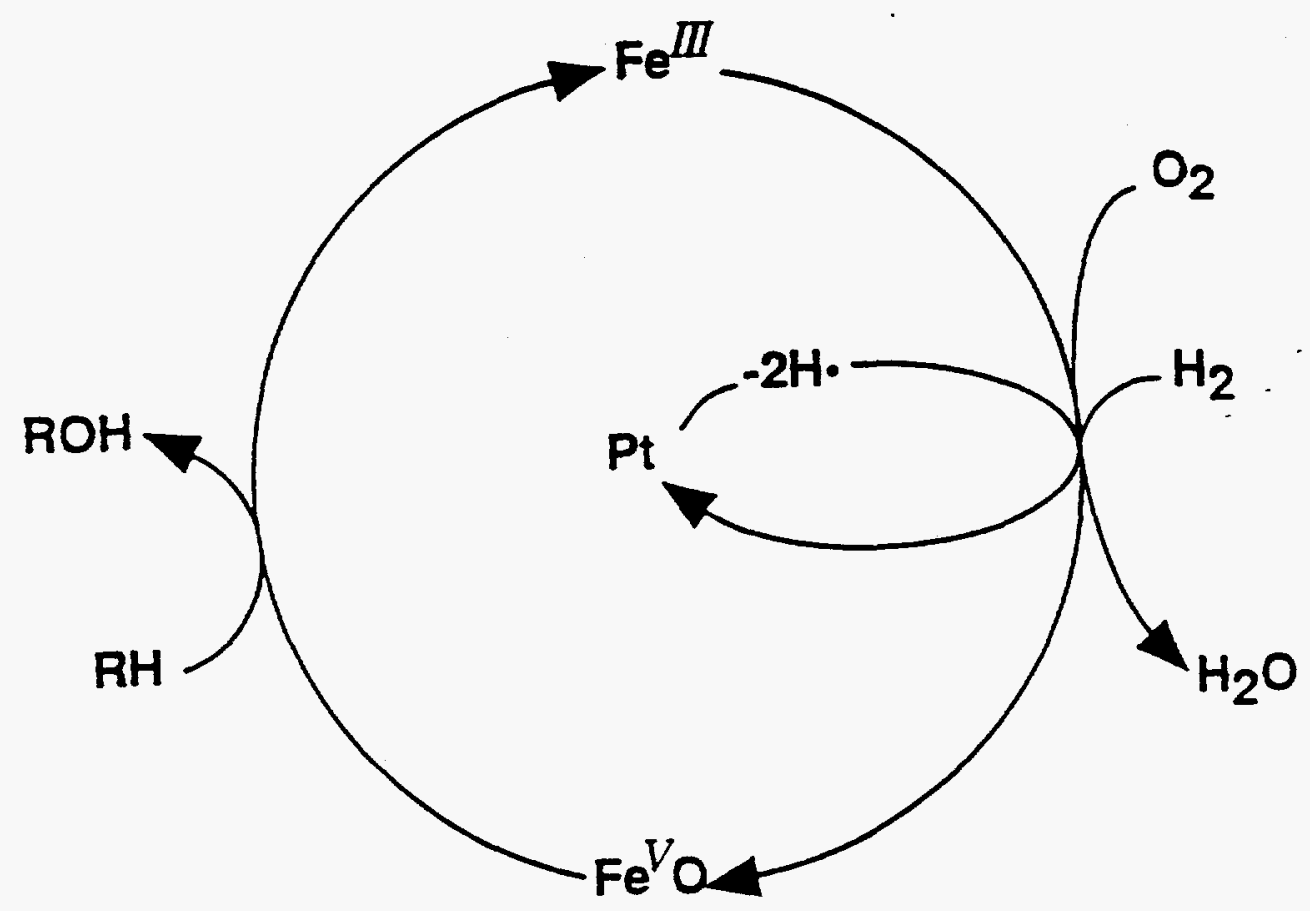


Figure 3 Alkane Hydroxylation with $\mathrm{O}_{2}$ Using a Suprabiotic Catalyst

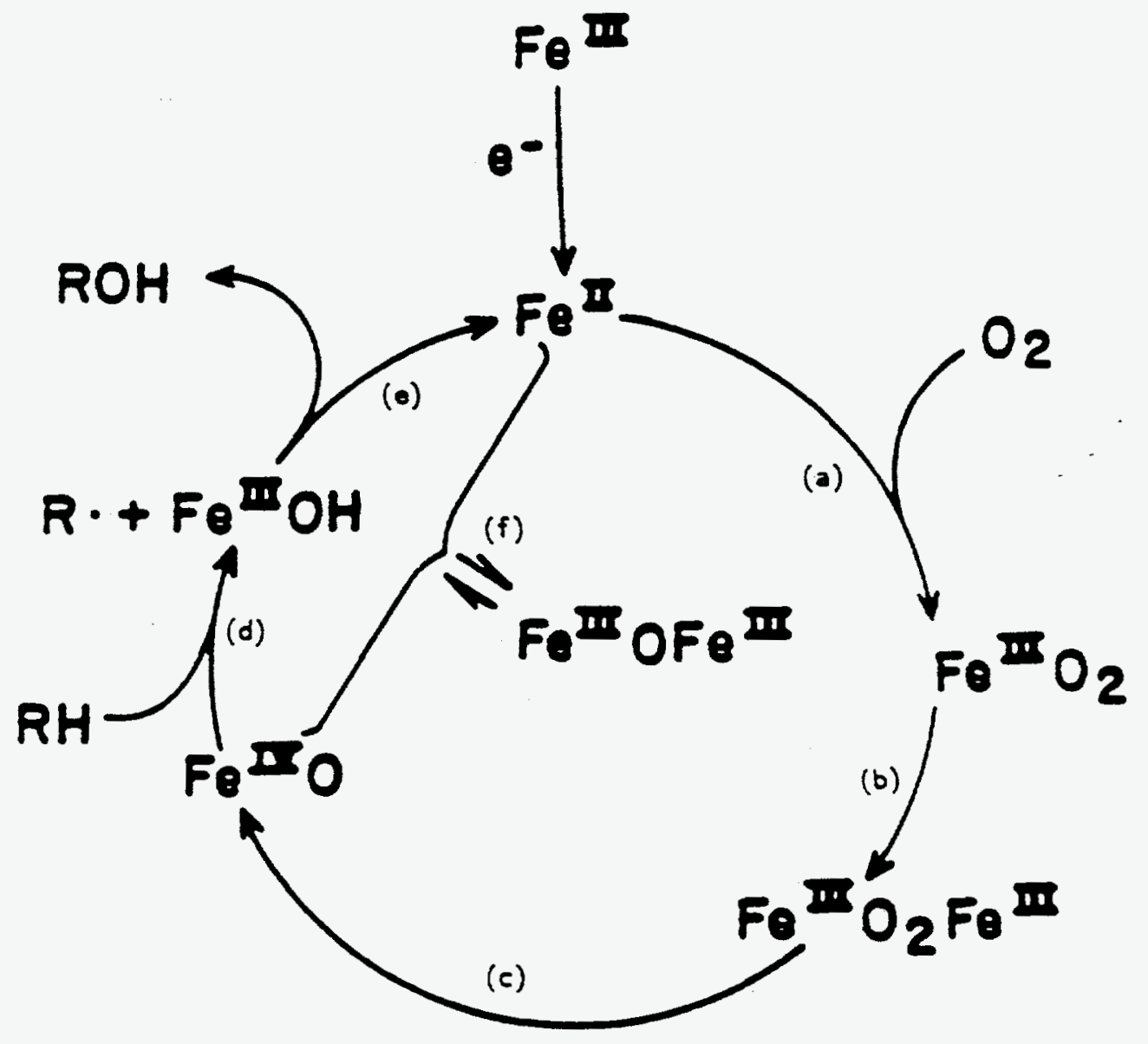




\section{PROPERTIES OF A SUPRABIOTIC CATALYST}

- COOPERATIVE IRON (II) SITES

$$
2 \mathrm{Fe}^{I I}+\mathrm{O}_{2} \longrightarrow \mathrm{Fe} \mathrm{II}_{2} \mathrm{Fe}^{I I I} \longrightarrow \mathrm{Fe}^{N_{\mathrm{O}}}
$$

- LIPOPHILIC ENVIRONMENT

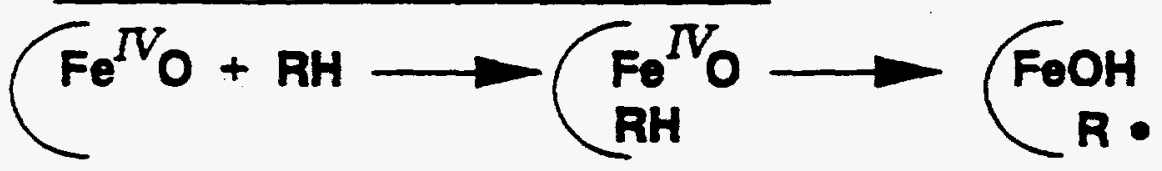

- HIGH Fe ${ }^{I I} / \mathrm{Fe}^{I}$ REDUCTION POTENTIAL

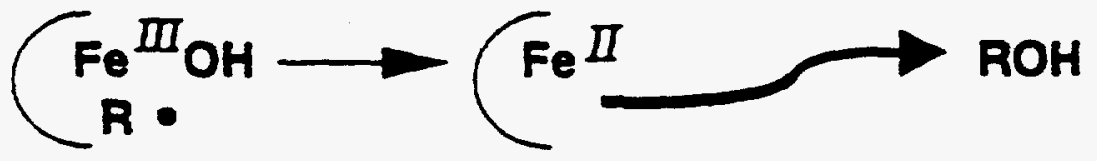

- HYDROPHOBIC ENVIRONMENT

- SUPPRESSED L OXO FORMATION

$\mathrm{Fe}^{I N O}+\mathrm{Fe}^{I I} \rightleftharpoons \mathrm{Fe}^{I I I} \mathrm{OFe}^{I I}$

- STABILITY TO OXIDATION

$$
\begin{aligned}
& \because \bullet \grave{F e}_{\mathrm{O}} \mathrm{V}_{\mathrm{O}} \longrightarrow\left[\mathrm{LFe}_{\mathrm{O}} \mathrm{m}_{\mathrm{O}}+\bullet\right. \\
& \mathrm{Fe} \mathrm{NO}_{\mathrm{O}}+\mathrm{LFe}^{x} \longrightarrow \mathrm{Fe} I+\mathrm{OLFe}^{x}
\end{aligned}
$$

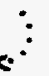


Figure 5 Substituted Porphyrin Macrocycle

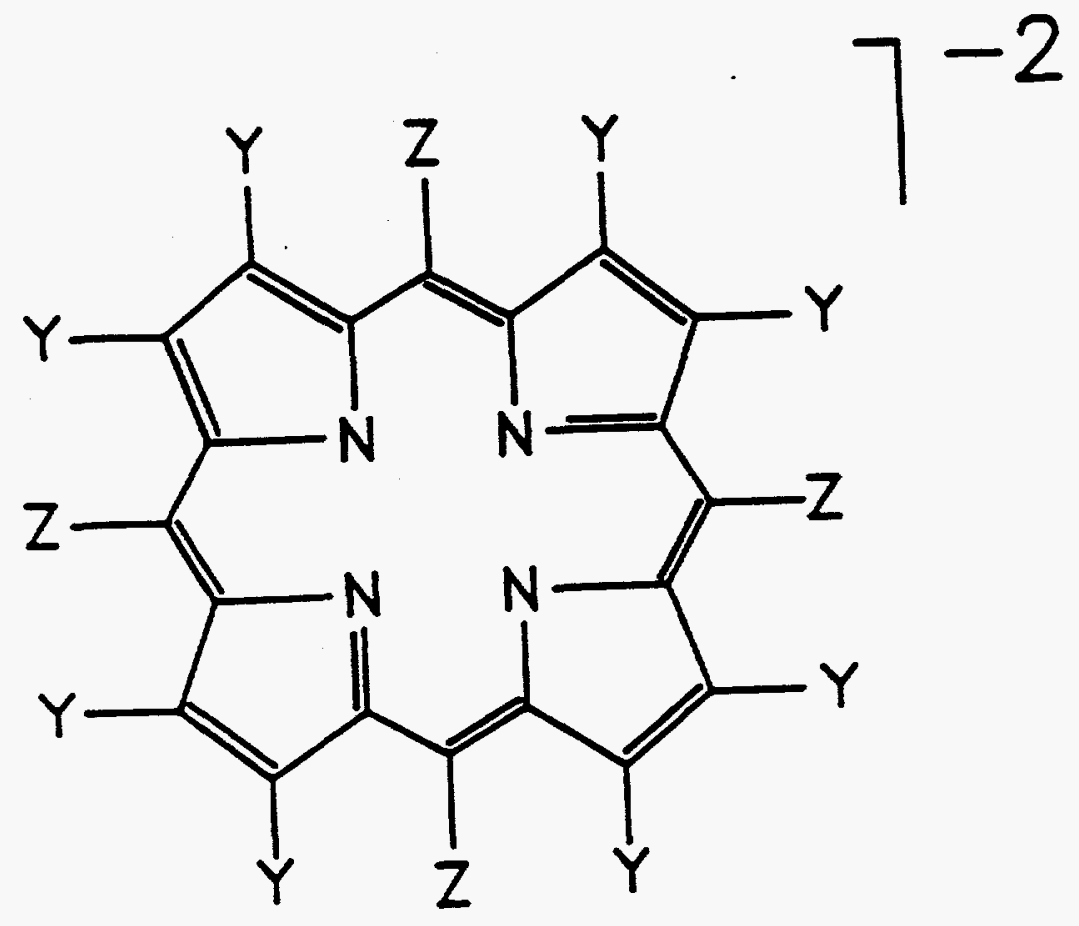




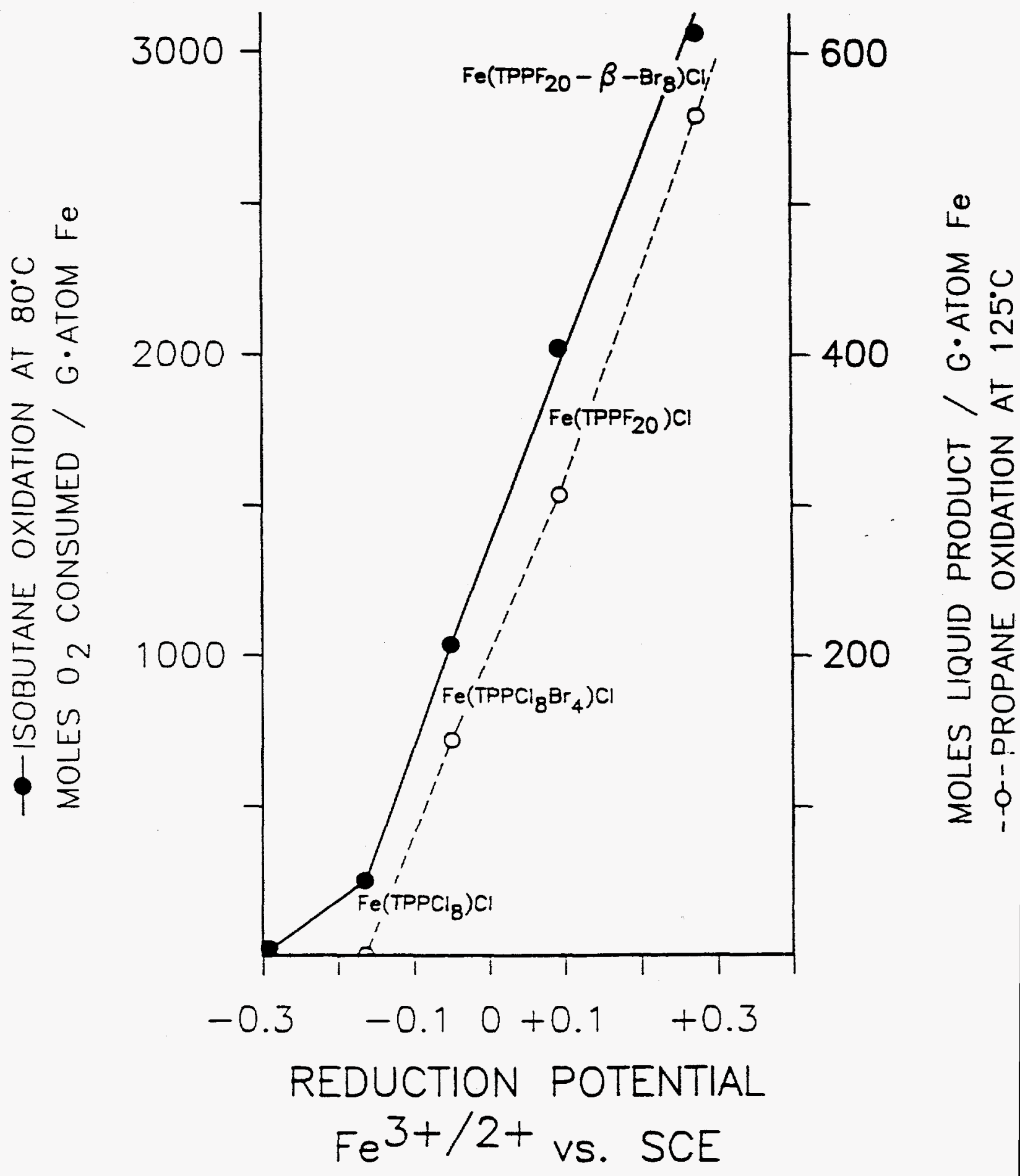




\section{0 FOR THE CATALYTIC OXIDATION OF ALKANES IN THE LIOUID PHASE}

\subsection{ABSTRACT}

Earlier work in our laboratories has shown that light alkanes can be air-oxidized using metalloporphyrin azides as catalysts. Phthalocyanine complexes are known to be more robust materials and cobalt phthalocyanine catalysts are used for mercaptan oxidations in the petroleum industry. Phthalocyanine complexes, however are not very hydrocarbon soluble nor very active for low temperature alkane oxidation. We report that chromium and manganese phthalocyanine azides catalyze selective oxidation of isobutane to tert-butyl alcohol and that iron perfluorophthalocyanine azides are robust, soluble, active catalysts for the selective oxidation of isobutane and propane under mild conditions of temperature and pressure.

\subsection{INTRODUCTION}

Light alkanes such as methane, ethane, propane and the butanes are among the most abundant and inexpensive raw materials available today. Often the search for oil yields extensive gas reservoirs. Gas availability on a worldwide scale is outstripping that of crude oil but problems of transportation and differences in end use application often limit its' utility. Light alkanes are made in refineries in growing amounts as the severity of processing is stepped up in order to convert increasingly heavy feeds. The utility of light alkanes in gasoline is now being severely curtailed because of vapor pressure restrictions on motor fuels.

However, not only are the light alkanes relatively cheap and abundant, they are among the most refractory of the hydrocarbon raw materials. Thus, selective, direct functionalization of these substrates has not been achieved in many instances. Oxidation of light $\left(C_{1}-C_{4}\right)$ alkanes is very difficult to stop short of complete combustion. Those selective commercial processes which have been developed for direct addition of molecular oxygen to these substrates produce carboxylic acids or carboxylic acid anhydrides as the major products [1]. There currently exists no direct one-step commercial process to convert a light alkane to an alcohol for use as a chemical or fuel product.

Recent work in our laboratories has shown that the first row azido(tetraphenylporphyrinato)metal(III) complexes, $\mathrm{M}$ (TPP) $\mathrm{N}_{3}(\mathrm{M}=\mathrm{Cr}, \mathrm{Mn}, \mathrm{Fe})$ are active catalysts for the selective formation of tert-butyl alcohol from isobutane and molecular oxygen at $80^{\circ} \mathrm{C}$. Over 250 turnovers were obtained after 6 hours using $\mathrm{Cr}(\mathrm{TPP}) \mathrm{N}_{3}$ as the catalyst [2]. This level of productivity exceeded that of conventional metal carboxylates or acetylacetonates and was equivalent to that obtained when the very active cobalt bis(pyridylimino)isoindolinato complexes $[3,4]$ were used. Although the azidometalloporphyrin complexes were active catalysts, the corresponding tetraphenylporphyrinatometal(III) halides and carboxylates were inactive [2]. In this note we report for the first time that azidometalphthalocyanine complexes of chromium, manganese and iron are active catalysts for the low temperature liquid phase oxidation of light alkanes whereas the corresponding metallophthalocyanine complexes from which they were derived are inactive. Since the phthalocyanine macrocycle is more robust than the porphyrin ring system under oxidation conditions, 
one incentive for studying these systems was to find active catalysts having a sturdier ligand system than the metalloporphyrins.

\subsection{RESULTS}

Metallophthalocyanine Azides. Azidometalphthalocyanine complexes, $M(P c) N_{3},[5],(M=C r$, $\mathrm{Mn}$,) are conveniently prepared from their phthalocyanato metal(II) precursors, $\mathrm{M}(\mathrm{Pc})$, by refluxing a saturated solution of sodium azide in ethanol over the $\mathrm{M}(\mathrm{Pc})$ precursor in a Soxhlet thimble. The $\mathrm{M}(\mathrm{Pc}) \mathrm{N}_{3}$-containing solution is evaporated to dryness and the solids washed with water and then dried. $\mathrm{Mn}(\mathrm{Pc}) \mathrm{N}_{3}, \mu \mathrm{N}-\mathrm{N}=2052 \mathrm{~cm}^{-1}$, UV/VIS [1-chloronaphthalene] (nm) 725,660 sh, 518; $\mathrm{Cr}(\mathrm{Pc}) \mathrm{N}_{3}, \mu \mathrm{N}-\mathrm{N}=2047 \mathrm{~cm}^{-1}$, UV/VIS [1-chloronaphthalene] (nm) 720, $660 \mathrm{sh}$. We were unable to prepare an iron phthalocyanine azide by reaction of the corresponding $\mathrm{Fe}(\mathrm{Pc})$ complex with sodium azide. Instead we obtained only the $\mu$-nitride, $[\mathrm{Fe}(\mathrm{Pc})]_{2} \mathrm{~N}[6]$.

Isobutane Oxidations Catalyzed by $\mathrm{PcMN}_{3}$. Table 1 shows that the azidometalphthalocyanine complexes, $\mathrm{PcMN}_{3}$, were active catalysts for the selective mild oxidation of isobutane to tert-butyl alcohol. The activity of these complexes was quite comparable to that of the analogous porphyrin [2]. The $\mu$-nitrido complex, $[\mathrm{Fe}(\mathrm{Pc})]_{2} \mathrm{~N}$, on the other hand was catalytically inactive for the low temperature oxidation of isobutane.

Perfluorophthalocyanatoiron Azides. Although we were unable to prepare an iron phthalocyanine azide from the corresponding $\mathrm{Fe}(\mathrm{Pc})$ complex, we were able to react perfluorophthalocyanato iron(II) [7] with sodium azide (ten-fold excess) in acetone over a- $48 \mathrm{hr}$. period in air at room temperature to produce the corresponding azide, $\mathrm{Fe}(\mathrm{FPc}) \mathrm{N}_{3}\left(\mathrm{vN}-\mathrm{N} 2037 \mathrm{~cm}^{-1}\right.$ ). UV/VIS [1-chloronaphthalene] (nm) $676,606 \mathrm{sh} ; \% \mathrm{C}, 42.7 ; \% \mathrm{~N}, 14.4 ; \% \mathrm{Fe} 6.8$; calc. for $\mathrm{Fe}(\underline{\mathrm{FPc}}) \mathrm{N}_{3}$ : $\% \mathrm{C} 42.7 ; \% \mathrm{~N} 17.1, \% \mathrm{Fe} 6.2$. This synthesis was poorly reproducible so we attempted an alternative metathetical reaction of the iron perfluorophthalocyanine chloride with sodium azide in methanol. This technique, however, appeared to produce the original azide only as a minor product and instead made a material having two azido bands in the ir (vN-N $1999 \mathrm{~cm}^{-1}$ and $\left.2030 \mathrm{~cm}^{-1}\right)$, perhaps the anionic diazido complex, $\mathrm{Na}\left[\mathrm{Fe}(\mathrm{FPc})\left(\mathrm{N}_{3}\right)_{2}\right]$, [8]. 
TABLE 1

\section{OXIDATION OF ISOBUTANE ${ }^{`}$ CATALYZED BY METAL PHTHALOCYANINE AZIDES}

\begin{tabular}{|c|c|c|c|c|c|}
\hline COMPLEX & mmoles & $\mathrm{T},{ }^{\circ} \mathrm{C}$ & thHrs. & TON & SEL \\
\hline $\mathrm{Mn}(\mathrm{Pc})$ & 0.013 & 80 & 6 & 0 & - \\
\hline $\mathrm{Mn}(\mathrm{Pc}) \mathrm{N}_{3}$ & 0.013 & 80 & 6 & 400 & 88 \\
\hline $\mathrm{Cr}(\mathrm{Pc}$ & 0.013 & 80 & 6 & 0 & - \\
\hline $\mathrm{Cr}(\mathrm{Pc}) \mathrm{N}_{3}$ & 0.013 & 80 & 7.5 & 220 & 91 \\
\hline $\mathrm{Fe}(\mathrm{Pc})$ & 0.030 & 80 & 6 & 0 & - \\
\hline$[\mathrm{Fe}(\mathrm{Pc})]_{2} \mathrm{~N}$ & 0.016 & 80 & 6 & 0 & - \\
\hline $\mathrm{Fe}(\underline{\mathrm{FPc}})$ & 0.023 & 80 & 6 & 0 & - \\
\hline $\mathrm{Fe}(\mathrm{FPc}) \mathrm{N}_{3}$ & 0.013 & 80 & 6 & 990 & 82 \\
\hline $\mathrm{Fe}(\underline{\mathrm{FPc}}) \mathrm{N}_{3}$ & 0.013 & 60 & 6 & 156 & 92 \\
\hline $\mathrm{Fe}(\mathrm{FPc}) \mathrm{Cl}$ & 0.013 & 80 & 6 & 260 & 86 \\
\hline $\begin{array}{l}\mathrm{Fe}(\mathrm{FPc}) \mathrm{Cl} \\
\left(+\mathrm{NaN}_{3}, 5 \mathrm{mg}\right)\end{array}$ & 0.013 & 80 & 6 & 650 & 84 \\
\hline \multicolumn{6}{|c|}{$\begin{array}{l}\text { 'The catalyst was stirred in } 25 \mathrm{ml} \text { benzene containing } 6 \text { grams isobutane in a Fisher-Porter aerosol tube } \\
\text { under } 100 \text { psig of } \mathrm{O}_{2} \text { at the designated temperature for the time indicated. Oxygen was admitted as used. } \\
\text { Products were analyzed by standardized g.c. }\end{array}$} \\
\hline \multicolumn{6}{|c|}{ Moles $\mathrm{O}_{2}$ consumed/mole catalyst used. } \\
\hline
\end{tabular}


Isobutane Oxidations Catalyzed by $F e(F P c) N_{3}$. The azido complex, $\mathrm{Fe}(\mathrm{FPc}) \mathrm{N}_{3}$, had very high activity for converting isobutane to tert-butyl alcohol, Table 1 . This complex was more soluble in benzene than any of the Pc complexes and appeared to have even greater stablility than the unfluorinated phthalocyanine complexes under oxidation conditions since solutions maintained their original deep color throughout the entire reaction period. Although more robust than the porphyrins, the phthalocyanines usually have the drawback of being much less soluble. Fluorination of the ligand seems to have had beneficial effects both on stabiity and solubility.

The diazido complex, tentatively formulated as $\mathrm{Na}\left[\mathrm{Fe}(\mathrm{EPc})\left(\mathrm{N}_{3}\right)_{2}\right]$, is also quite active for the oxidation of both isobutane and propane. Furthermore the iron phthalocyanine chloride, $\mathrm{Fe}(\mathrm{FPc}) \mathrm{Cl}$, from which it was prepared is also active, using 260 mmoles of oxygen per mmole of catalyst in six hours and producing tert-butyl alcohol in $86 \%$ selectivity. If sodium azide is added to the benzene solution of $\mathrm{Fe}(\mathrm{FPc}) \mathrm{Cl}$, prior to oxidation, the activity increases and 650 mmoles of oxygen per mmole of catalyst is taken up, Table 1. It appears that an active azide may have been formed $\underline{\underline{n}}$ situ in this case.

Other Light Alkane Oxidations. Encouraged by the high activity of perfluorophthalocyanatoiron(II)azide for isobutane oxidation, we tried this complex as a catalyst for the air-oxidation of propane - a more difficult substrate to oxidize. Propane is oxidized to a mixture of isopropyl alcohol and acetone at $125^{\circ} \mathrm{C}$ using $\mathrm{Fe}(\mathrm{FPc}) \mathrm{N}_{3}$ as a catalyst, Table 2 . The reaction is quite selective to the $C_{3}$ oxygenates and low yields of carbon oxides or liquid by-products are observed. Even refractory hydrocarbons such as methane and ethane can be oxidized in the presence of $\mathrm{Fe}(\mathrm{FPc}) \mathrm{N}_{3}$ but rates, yields and selectivity to alcohols are very low. Methane is oxidized at $285^{\circ} \mathrm{C}$ and 2000 psig of a 3/1 mixture of methane and air in 1/1 benzene/water to give methanol in $11 \%$ selectivity at $60 \%$ oxygen conversion using this catalyst. By-products are largely carbon oxides.

\subsection{DISCUSSION}

The use of phthalocyanine complexes as catalysts for light alkane oxidation is in some ways analogous to the porphyrin catalyzed oxidations reported earlier $[2,9,10,11]$. Both fluorination of the macrocycle and the presence of an axial ligand result in increased catalytic activity. In both cases $\mu$-nitrido species are not active.

We have shown in an earlier report [2] a similar azide promotion of a bis(pyridylimino)isoindolinatocobalt complex, $\mathrm{Co}(\mathrm{BPI}) \mathrm{N}_{3}$, which has activity comparable to that of the alkylperoxo complex, $\mathrm{Co}(\mathrm{BPI}) \mathrm{OOR}$, and is far more active than the acetate, Co(BPI)OAc. Thus, though difficult to understand, azide promotion of the oxidation activity of macrocyclic first row metal complexes appears to be somewhat general.

One might expect that expulsion of the azide radical from a metal(III) complex could create the metal(II) species which could coordinate and possibly activate molecular oxygen. Loss of azide radical occurs during pyrolysis of porphyrinatometal azides [14], Eq. 1, and may well occur from the coordination sphere of other metal azide complexes at lower temperatures in solution. 
TABLE 2

\section{OXIDATION OF PROPANE ${ }^{a}$ CATALYZED BY AN IRON PERFLUOROPHTHALOCYANINE AZIDE COMPLEX}

\begin{tabular}{|c|c|c|c|c|c|c|}
\hline COMPLEX & mmoles & SOLVENT & $\mathrm{T},{ }^{\circ} \mathrm{C}$ & T,HRS. & $\mathrm{TON}^{\mathrm{b}}$ & IPA/ACETON \\
\hline $\mathrm{Fe}(\underline{\mathrm{FPc}})$ & 0.023 & Benzene & 125 & 3 & 0 & - \\
\hline $\mathrm{Fe}(\mathrm{FPc}) \mathrm{N}_{3}$ & 0.028 & Benzene & 125 & 6 & 48 & 0.7 \\
\hline $\mathrm{Fe}(\underline{\mathrm{FPc}})$ & 0.023 & $\mathrm{CH}_{3} \mathrm{CN}$ & 125 & 3 & 57 & 0.7 \\
\hline $\mathrm{Fe}(\underline{\mathrm{FPc}})+\mathrm{NaN}_{3}^{\mathrm{d}}$ & 0.028 & $\mathrm{CH}_{3} \mathrm{CN}$ & 125 & 3 & 98 & 0.7 \\
\hline $\mathrm{Fe}(\mathrm{FPc})$ & 0.028 & $\mathrm{CH}_{3} \mathrm{CN}$ & 150 & 3 & 460 & 0.3 \\
\hline $\mathrm{Fe}(\mathrm{EPc})+\mathrm{NaN}_{3}^{\mathrm{d}}$ & 0.028 & $\mathrm{CH}_{3} \mathrm{CN}$ & 150 & 3 & 567 & 0.4 \\
\hline $\mathrm{Fe}(\underline{\mathrm{FPc}}) \mathrm{N}_{3}$ & 0.028 & $\mathrm{CH}_{3} \mathrm{CN}$ & 150 & 3 & 754 & 0.7 \\
\hline $\mathrm{Fe}(\mathrm{FPc}) \mathrm{N}_{3}$ & 0.028 & $\mathrm{C}_{6} \mathrm{H}_{5} \mathrm{CN}$ & 150 & 3 & 1210 & 0.6 \\
\hline
\end{tabular}

'Propane ( $1.36 \mathrm{~mol})$ was added to the solvent $(48 \mathrm{ml})$ containing the catalyst. The reaction mixture was stirred at the designated temperature under 1000 psig of air in a glass-lined autoclave. Liquids and gases were analyzed by g.c. Production of carbon oxides never exceeded $10 \%$ of total products. Isopropyl alcohol and acetone exceeded 85 mole \% of carbon-containing reaction products in all cases.

Moles of acetone plus isopropyl alcohol formed per mole of catalyst used.

Molar ratio of isopropyl alcohol to acetone formed.

${ }^{\mathrm{d}}$ Sodium azide, $5 \mathrm{mg}$, was added prior to run. 


$$
\mathrm{M}(\mathrm{TPP}) \mathrm{N}_{3} \rightleftharpoons \mathrm{M}(\mathrm{TPP})+\mathrm{N}_{3}^{\bullet}
$$

This explanation appears to be at odds with the low reactivity of the metal(II)phthalocyanine complexes described above. Most Metal(II) phthalocyanines are quite insoluble, however, and this may contribute to their apparent low activity under very mild conditions. An alternative explanation might be that reaction between azide radical and molecular oxygen produces nitrogen oxides which aid oxidation by forming reactive metal oxo intermediates. Work continues in an attempt to shed more light on this very interesting and general phenomenon of azide promotion of metal catalyzed alkane oxidations.

\subsection{CONCLUSIONS}

Phthalocyanines have been used as catalysts for a wide variety of oxidations [12] and in particular the cobalt phthalocyanines have found widespread use in the well known Merox process [13] for mercaptan oxidation in the petroleum industry. The use of Pc complexes for alkane oxidations as well as the promotion of these reactions by azido ligands or by macrocyclic ring fluorination have not been exploited. The relatively high rates of selective alkane oxidation due to the presence of the azide ligand, together with increases both in catalyst solubility and stability due to a perfluoro ligand, are all of interest with respect to the eventual production of a practical catalyst.

Research continues in this area in hopes of creating a robust, soluble, active catalyst for selective alkane oxidation.

\subsection{REFERENCES}

1. J. E. Lyons, "Surface Organometallic Chemistry: Molecular Approaches to Surface Catalysis", ed. J. M. Basset, Kluwer Academic Publishers, 1988, pp 100-101.

2. P. E. Ellis, Jr. and J. E. Lyons, J.C.S. Chem. Commun., 1989, 1187.

3. L. Saussine, E. Brazi, A. Robine, H. Mimoun, J. Fischer, and R. Weiss, J. Am. Chem Soc., $1985,107,3534$.

4. C. A. Tolman, J. P. Druliner, P. J. Krusic, M. J. Nappa, W. C. Seidel, I. D. Williams and S. D. Ittel, J. Mol. Catal., 1988, 48, 129.

5. V.L. Goedken and C Ercolani, J.C.S. Chem. Commun., 1984, 378.

6. P.E. Ellis, Jr., J.E. Lyons, and H.K. Myers, Jr., U.S. Patent 5,093,491, March 3, 1992.

7. J.G. Jones and M.V. Twigg, Inorg. Chem., 1969, 8, 2018.

8. B. J. Kennedy, K.S. Murray, P.R. Zwack, H. Hamborg and W. Katz, Inorg. Chem., 1986, 25, 2539. 


\subsection{REFERENCES (CONIINUED)}

9. P. E. Ellis, Jr. and J. E. Lyons, J.C.S. Chem. Commun., 1989, 1189.

10. P. E. Ellis, Jr. and J. E. Lyons, J.C.S., Chem. Commun., 1989, 1315.

11. P. E. Ellis, Jr. and J. E. Lyons, Catalysis Letters, 1989, 3, 389.

12. M. Rhonec and J. Ilavsky, Petrochemia, 1983, 23, 89.

13. A. Leitao and A. Rodrigues, Chem. Eng. Sci., 1988, 44, 1245.

14. H. Sugaya, J. Tachiband and T. Imamura, Polyhedron, 1991, 10, 373. 


\subsection{HALOGEN SUBSTTUENT EEEECTS ON THE CATALYTIC ACTIVTYY OF IRON PORPHYRIN COMPLEXES FOR THE DECOMPOSIULN OF tert-BUTYL HYDROPEROXIDE}

\subsection{INTRODUCTION}

It has long been known that iron complexes are effective promoters of the decomposition of alkyl hydroperoxides in the liquid phase (1). There has been considerable recent interest in the efficient catalytic conversion of tert-butyl hydroperoxide to tert-butyl alcohol (2-12), Eq. 1, but with currently available catalyst systems, elevated temperatures are often required and reaction selectivity is typically around $80 \%$. Tetraphenylporphyrinatoiron(III)chloride was recently shown to be one of the more active catalysts for decomposition of tert-butylhydroperoxide (4), but in order to obtain relatively rapid rates it was necessary to either conduct reactions at somewhat elevated temperatures $\left(60^{\circ} \mathrm{C}\right)$ or to add imidazole which resulted in a hydroperoxide decomposition catalyst that was active at room temperature.

$$
\mathrm{ROOH} \longrightarrow \mathrm{ROH}+1 / 2 \mathrm{O}_{2}
$$

In this communication we report that by halogenating the porphyrin ring of iron tetraphenylporphyrinato complexes we were able to greatly enhance catalytic activity, reduce reaction temperatures and observe reaction selectivities of $90 \%$. Perhaloporphyrinatoiron(III) complexes gave the fastest rate of hydroperoxide decomposition by any metal complex observed to date. In fact, with room temperature reaction rates of well over 100 catalyst cycles per second, these are among the fastest reactions catalyzed by a synthetic homogeneous transition metal complex in solution (14). Rates are within an order of magnitude of the activity of the catalase enzyme which decomposes hydrogen peroxide to water and oxygen (15). In addition, this study may have relevance to the mechanism of some recently disclosed rapid air-oxidations of isobutane catalyzed by iron haloporphyrin catalysts $(16,17)$.

\subsection{EXPERIMENTAL}

Materials. Metal complexes used as catalysts:tetrakispentafluorophenyl $\beta$-octachloroporphyrinatoiron(III)chloride, $\mathrm{Fe}\left(\mathrm{TPPF}_{20} \beta-\mathrm{Cl}_{8}\right) \mathrm{Cl}$,tetrakispentafluorophenyl $\beta$-octabromoporphyrinatoiron(III)chloride, $\mathrm{Fe}\left(\mathrm{TPPF}_{20} \beta-\mathrm{Br}_{8}\right) \mathrm{Cl}$, and tetrakispentafluorophenylporphyrinatoiron(III)chloride, $\mathrm{Fe}\left(\mathrm{TPPF}_{20}\right) \mathrm{Cl}$, were prepared by methods described previously $(16,17)$. Benzene, $(99.99 \%)$, Aldrich, tert-butyl alcohol, (99.6\%), Aldrich, and p-xylene, $(99+\%)$, Aldrich were used as purchased. tert-Butyl hydroperoxide $(90 \%, 5 \%$ water, $5 \%$ tert-butyl alcohol) was used as purchased from Aldrich. 
Procedures. A solution of the catalyst in p-xylene (internal standard) was quickly added to a rapidly stirring solution of tert-butyl hydroperoxide in the solvent at room temperature. Oxygen evolution was measured manometrically and liquid products were analyzed periodically by standardized gas chromatography.

\subsection{RESULTS}

Figure 1 compares the activity of a number of iron complexes for the decomposition of tert-butyl hydroperoxide, TBH, in benzene. It is clear that porphyrinato iron(III) complexes are quite active for catalyzing this transformation and that the extent of porphyrin ring halogenation has a dramatic effect on the catalytic activity of the porphyrinato iron(III) complex used. Very low concentrations of the perhaloporphyrin complexes $\mathrm{Fe}\left(\mathrm{TPPF}_{20} \beta-\mathrm{Y}_{8}\right) \mathrm{Cl}, \mathrm{Y}=\mathrm{Cl}, \mathrm{Br}$ were extremely effective in catalyzing room temperature TBH decomposition. Table 1 compares the product profile and extent of conversion of TBH in benzene after two or three hours. Selectivity to tert-butyl alcohol, TBA, was found to be $90 \%$ in all instances in which perhaloporphyrinato complexes were used as catalysts. Selectivity was lower when partially halogenated or unhalogenated porphyrins were used.

Kropf and Ivanov recently observed second order dependence on hydroperoxide concentration during some copper phthalocyanine complex-catalyzed decompositions of tert-butyl hydroperoxide (12) carried out at $60^{\circ} \mathrm{C}$ in chlorobenzene. Interestingly, these workers found that ele tron donation to the phthalocyanine macrocycle enhanced catalytic activity whereas electron withdrawal retarted it. Figure 2 shows the apparent second order dependence on hydroperoxide concentration exhibited by decompositions catalyzed by both halogenated and unhalogenated tetraphenylporphyrinatoiron(III)chloro complexes in benzene as a solvent. Incremental addition of the radical inhibitor, 2,4-di-tert-butyl-p-cresol, prolonged an induction period which grew longer by a time that was proportional to the concentration of inhibitor used, Figure 3. At inhibitor concentrations 10,000 fold greater than that of the iron porphyrin complex, the induction period was still less than one hour long.

\subsection{DISCUSSION}

There has been much discussion in the recent literature $(9,13,18)$ surrounding the issue of heterolytic vs. homolytic $\mathrm{O}-\mathrm{O}$ bond cleavage in hydroperoxides during metalloporphyrin catalyzed hydroperoxide decomposition. It has been shown that tert-butyl hydroperoxide undergoes homolytic $\mathrm{O}-\mathrm{O}$ bond cleavage in the presence of porphyrinato iron complexes $(18,19,20)$. Peracidic compounds undergo heterolytic cleavage under the same conditions (18). A recent report (13) indicates that electron "push" from electron donating substituent groups on the macrocycle coupled with electron "pull" from the peroxide bound to an iron in the macrocycle result in enhanced rate of heterolytic $\mathrm{O}-\mathrm{O}$ bond cleavage, Eq. 2. Electron pull from the bound peroxide is generated by electon withdrawing groups, $\mathrm{Y}(\mathrm{p})$, in the p-position on the aromatic peracids used in the study. These observations are reasonable since by pushing electron density from the macrocylic ligand toward the iron center one might stabilize a high-oxidation state ferryl and by removing electron density from the aromatic peracid portion of the complex one would stabilize developing negative charge. 


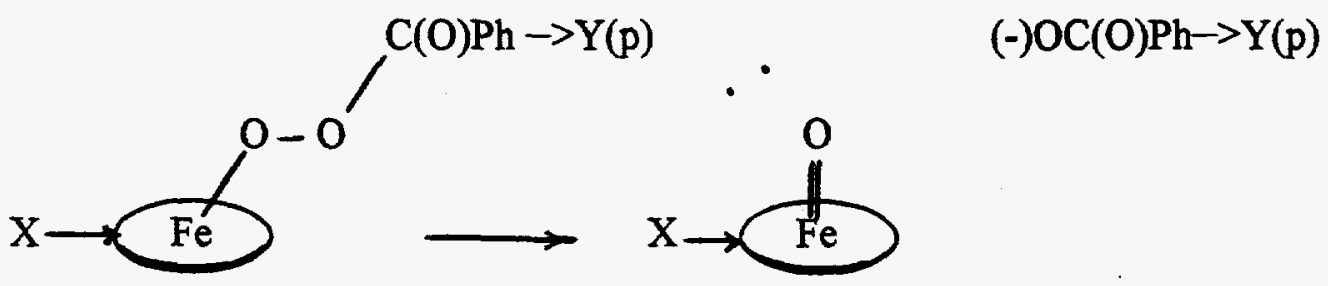

The studies reported in this communication demonstrate the effects of electron "pull" from the porphyrin macrocycle and electron "push" from the alkyl-hydroperoxide that is decomposed. Surprisingly, the result is that of greatly enhanced decomposition rates. These observations could be rationalized by a mechanism shift to homolytic $\mathrm{O}-\mathrm{O}$ bond cleavage, Eq. 3, which is enhanced by electron withdrawal from the macrocyclic ligand and thus from the metal center which it surrounds. Electron withdrawal would be expected to raise the $\mathrm{Fe}(\mathrm{III}) / \mathrm{Fe}(\mathrm{II})$ reduction potential $(16,17)$ and favor the homolytic $\mathrm{O}-\mathrm{O}$ bond dissociation step resulting in the production of iron(II) from the iron(III) peroxy complex with release of alkylperoxy radicals. The resulting tert-butylperoxy radical could react further to produce oxygen and the tert-butoxy radical, Eq. 4, which ultimately gives TBA $(9,12,18)$.

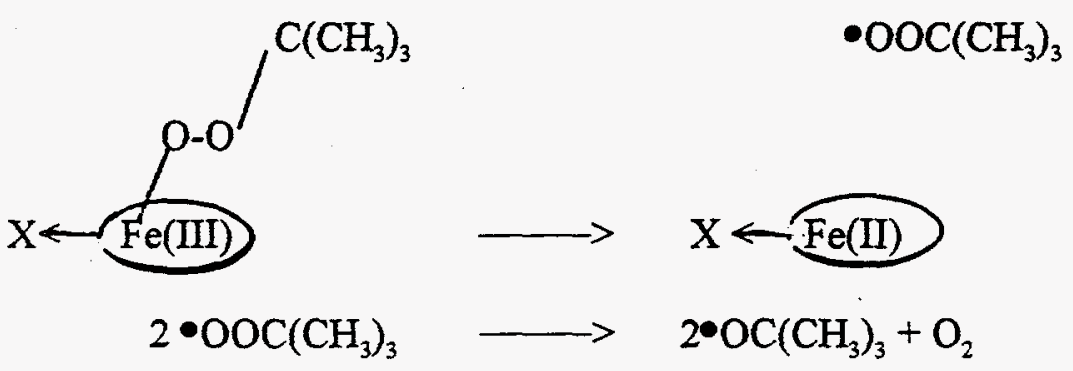

A catalytic reaction which is consistent with the observations presented above can be envisioned as proceeding via the pathway shown in Scheme 1, below. Early studies of metal complex catalyzed hydroperoxide decomposition were rationalized based on $\mathrm{M}(\mathrm{III}) / \mathrm{M}(\mathrm{II})$ catalysis via the well known Haber-Weiss cycle shown in Scheme 1.

More recent studies, however, have indicated that metal oxo species may be involved (18-21) in metalloporphyrin-catalyzed alkylhydroperoxide decomposition. Balch and his co-workers have found (19) that $\mathrm{Fe}(\mathrm{TPP}) \mathrm{OH}$ reacts stoichiometrically with tert-butylhydroperoxide to form the alkylperoxy iron(III) complex suggested in Scheme 1. In the presence of a suitable base (19) this complex homolyzes to form not Fe(II), but the ferryl complex, Eq.5. Scheme 2 shows the possible generation of alkoxy and alkylperoxy radicals by a mechanism in which no iron(II) species take part, but rather the catalysis occurs via a one-electron homolytic $\mathrm{Fe}(\mathrm{III}) / \mathrm{Fe}(\mathrm{IV})=0$ couple. At this time it is not possible to assess the relative contributions from pathways shown in Schemes 1 and 2.

$$
(\mathrm{TPP}) \mathrm{FeOO}(\mathrm{t}-\mathrm{Bu})+\mathrm{B} \longrightarrow(\mathrm{B})(\mathrm{TPP}) \mathrm{Fe}(\mathrm{IV})=\mathrm{O}+\mathrm{t}-\mathrm{BuO}
$$


The similarity between the effects of reduction potential on catalytic activity for both the decomposition of tert-butyl hydroperoxide to tert-butyl alcohol and the air-oxidation of isobutane (16, 17) to tert-butyl alcohol raises the possibility that these reactions have pathways in common. We continue to pursue the synthetic potential of this reaction, extend the trends in catalytic activity with increasing reduction potential, and consider possible implications of this work for air oxidations using perhaloporphyrin catalysts.

\subsection{REFERENCES}

1. Sosnovsky, G. and Rawlinson, D.J., "Organic Peroxides", D. Swern, Ed. Wiley, New York, pp 153-268, 1971.

2. J.R. Sanderson, E.T. Marquis, U.S. Patent 4,922,035, May 1, 1990.

3. Sanderson, J.R., Marquis, E.T. and Knifton, J.F., U.S. Patent 4,922,036, May 1, 1990.

4. Sanderson, J.R., Marquis, E.T. and Mueller, M.A., U.S. Patent 4,922,034, May 1, 1990.

5. Sanderson, J.R., Knifton, J.F. and Marquis, E.T., U.S. Patent 4,922,033, May 1, 1990.

6. Sanderson, J.R., Knifton, J.F., Marquis, E.T. and Mueller, M.A., U.S. Patent 4,912,256, Mar. 27, 1990.

7. Sanderson, J.R. and Knifton, J.F., U.S. Patent 4,910,349, Mar. 20, 1990.

8. Sanderson, J.R., U.S. Patent 4,712,267, Mar. 27, 1990.

9. Bruice, T.C., Acc. Chem. Res., 24, 243 (1991).

10. Barton, D.H.R., Beviere, S.D., Chavasiri, W., Csuhai, E., Doller, D., and Liu, W.G., I. Amer. Chem. Soc, 114.2147 (1992).

11. Sawada, H., Shigaki, H. Kato, M and Nakayama, M., Bull Chem. Soc. Jpn, 64, 785 (1991).

12. Kropf., H. and Ivanov, S.K., Oxidation Commun, 13, 23 (1990).

13. Yamaguchi, K., Watanabe, Y., and Morishima, I., Inorg. Chem., 31, 157 (1992).

14. Parshall, G.W., "Homogeneous Catalysis", Wiley, N.Y., p.177, 1980.

15. Hoffmann, P. and Meunier, R.B., Bull. Soc. Chim. Fr., 129, 85 (1992). 
16. Lyons, J.E. and Ellis, P.E., Jr., Catalysis Letters, $\underline{8}, 45$ (1991).

17. Ellis, P.E., Jr. and Lyons, J.E., Coord. Chem. Rev, 105, 181 (1990).

18. Lee, W.E. and Bruice, T.C., L.Amer. Chem. Soc., 107, 513 (1985).

19. Balch, A.L., Hart, R.L., Latos-Grazynski, L, and Traylor, T.G., J.Amer Chem Soc, $112,7382(1990)$.

20. Traylor, T.G. and Xu, F., I Amer. Chem. Soc., 109, 6201 (1987). 


\section{TABLE 1}

CONVERSION OF tert-BUTYL HYDROPEROXIDE TO tert-BUTYL ALCOHOL,

G

TME $\quad \mathrm{t}^{-\mathrm{BuO}_{2}} \mathrm{H} \quad-$ PRODUCT, MOLAR SEL., $\%$ -

CATALYST

Hrs

CONV.,\%

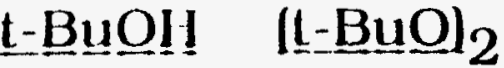

$\left.\mathrm{ICH}_{3}\right)_{2}$ COQ

$\mathrm{Fe}(\mathrm{ACAC})_{3}$

2.3

$<5$

67

$\operatorname{tr}$

32

Fe(TPP)Cl

1.9

27

82

7

11

$\mathrm{Fe}\left(\mathrm{TPPF}_{20}\right) \mathrm{Cl}$

3.3

72

87

10

3

$\mathrm{Fe}\left(\mathrm{TPPF}_{20} \boldsymbol{\beta - B r _ { 8 }}\right) \mathrm{Cl} \quad 1.9$

95

90

8

2

$\mathrm{Fe}\left(\mathrm{TPPF}_{20} \beta-\mathrm{Cl}_{8}\right) \mathrm{Cl} \quad 3.3$

100

90

8

2

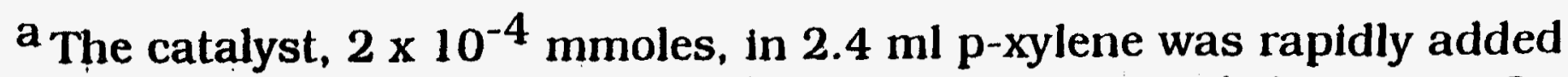
to a stirred solution of $10 \mathrm{ml} \mathrm{t}-\mathrm{BuO}_{2} \mathrm{H}(90 \%)$ in $48 \mathrm{mls}$ benzene. $\mathrm{O}_{2}$ evolved was measured manometrically and liquid samples taken periodically and analyzed by standardized gc. 
FIGURE 1 Formation of tert-butyl alcohol, TBA, from tert-butyl hydroperoxide $(10 \mathrm{ml})$ in benzene $(48 \mathrm{ml})$-p-xylene $(2.4 \mathrm{ml})$ using $2 \times 10^{-4}$ mmoles of catalyst:

$\bigcirc \mathrm{Fe}\left(\mathrm{TPPF}_{20} \mathrm{\beta}-\mathrm{Cl}_{8}\right) \mathrm{Cl}, \triangle \mathrm{Fe}\left(\mathrm{TPPF}_{20}\right) \mathrm{Cl}, \bullet \mathrm{Fe}(\mathrm{TPP}) \mathrm{Cl}, \triangle \mathrm{Fe}(\mathrm{acac})_{3}$.

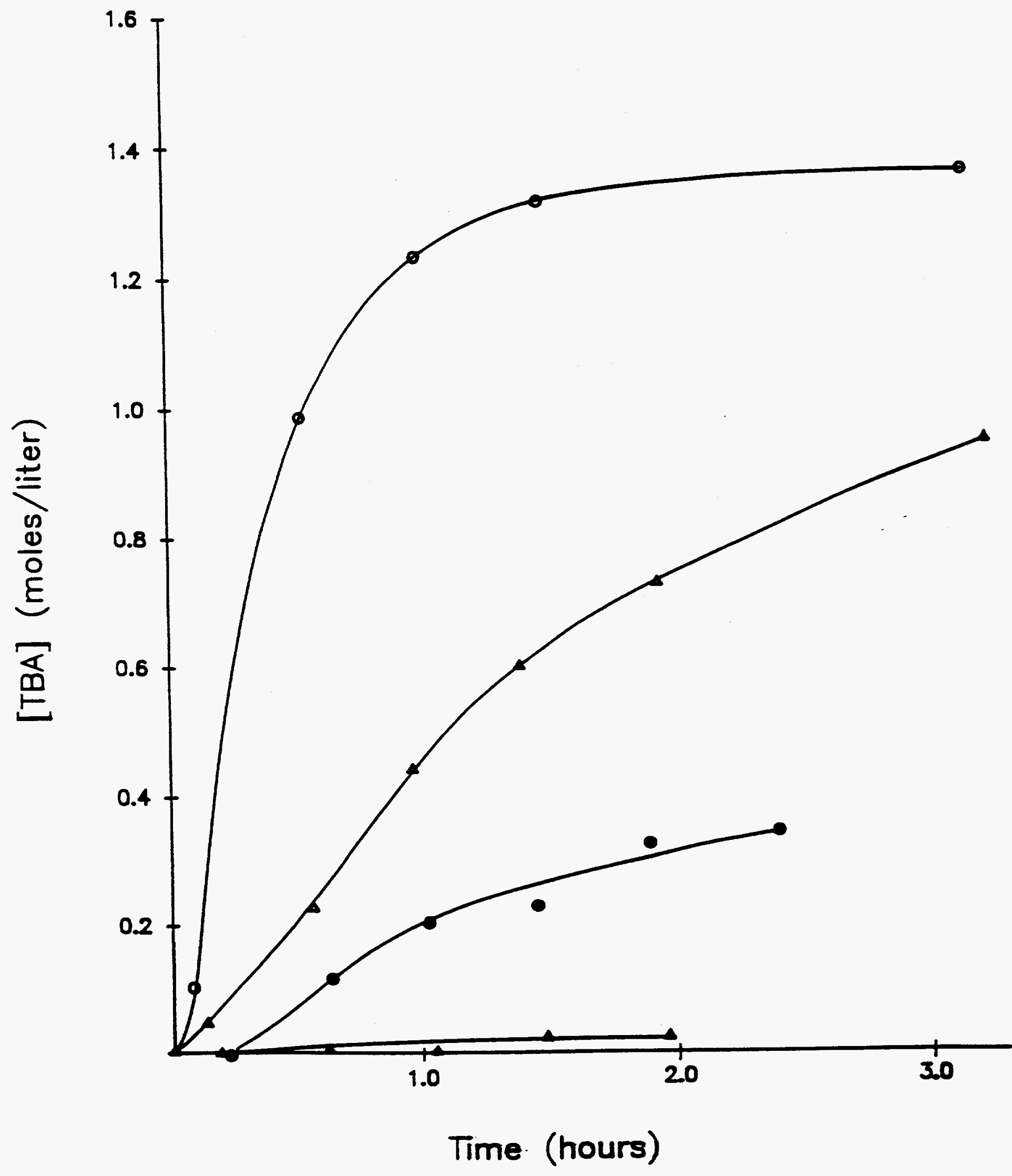


Figure 2

Second order plots for the catalytic decomposition of tert-butyl hydroperoxide in benzene $\diamond \mathrm{Fe}\left(\mathrm{TPPF}_{20} \mathrm{\beta}-\mathrm{Cl}_{8}\right) \mathrm{Cl}, \quad \triangle \mathrm{Fe}\left(\mathrm{TPPF}_{20}\right) \mathrm{Cl}, \quad \mathrm{OFe}(\mathrm{TPP}) \mathrm{Cl}$.

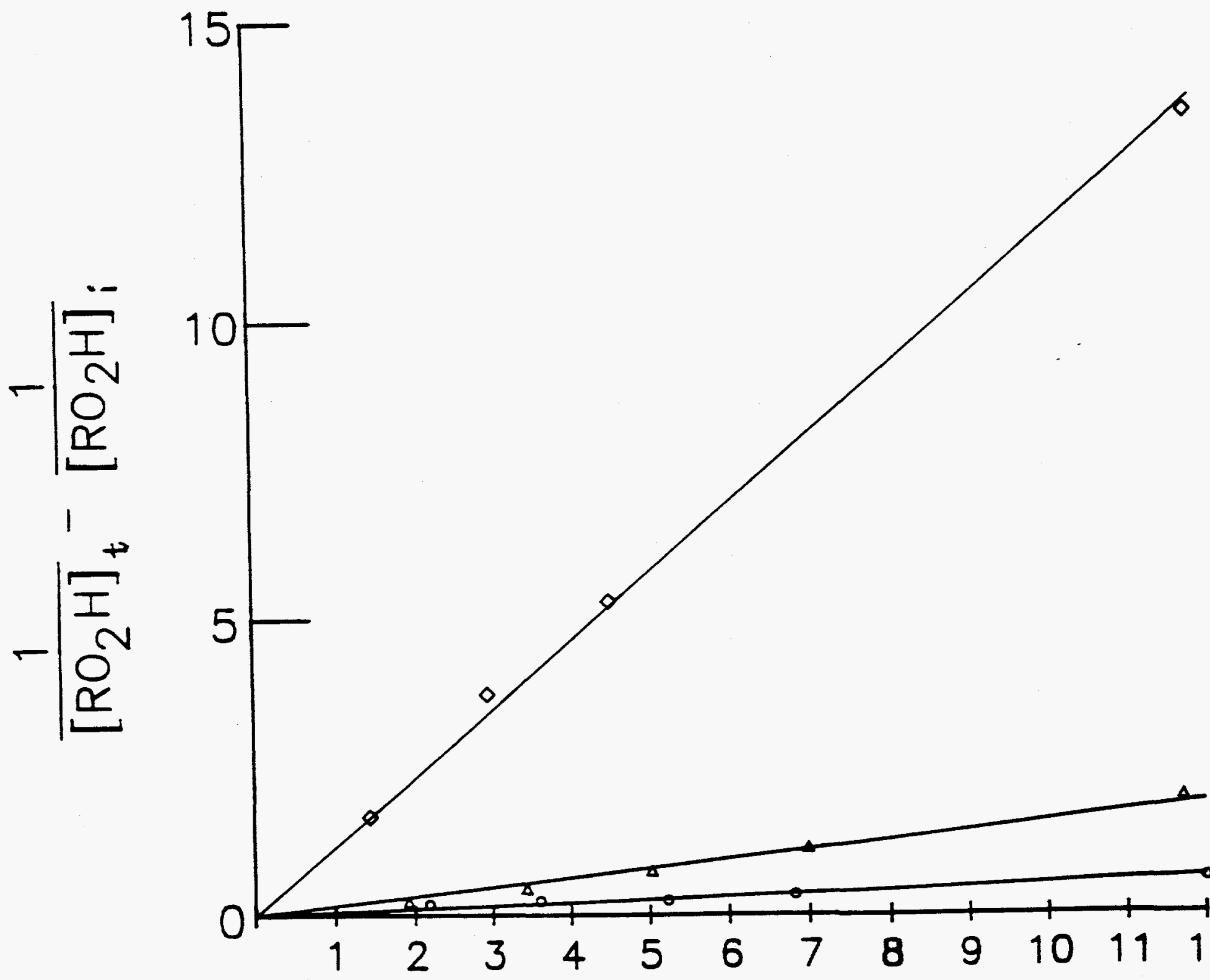

Time (seconds $\left.\times 10^{3}\right)$ 


\section{Figure 3}

Effect of BHT on the decomposition of tert-butyl hydroperoxide $(10 \mathrm{ml})$ catalyzed by $\mathrm{Fe}\left(\mathrm{TPPF}_{20} \beta-\mathrm{Cl}_{8}\right) \mathrm{Cl}, 2 \times 10-4$, mmoles, in benzene $(48 \mathrm{ml})$-p-xylene $(2.4 \mathrm{ml})$.

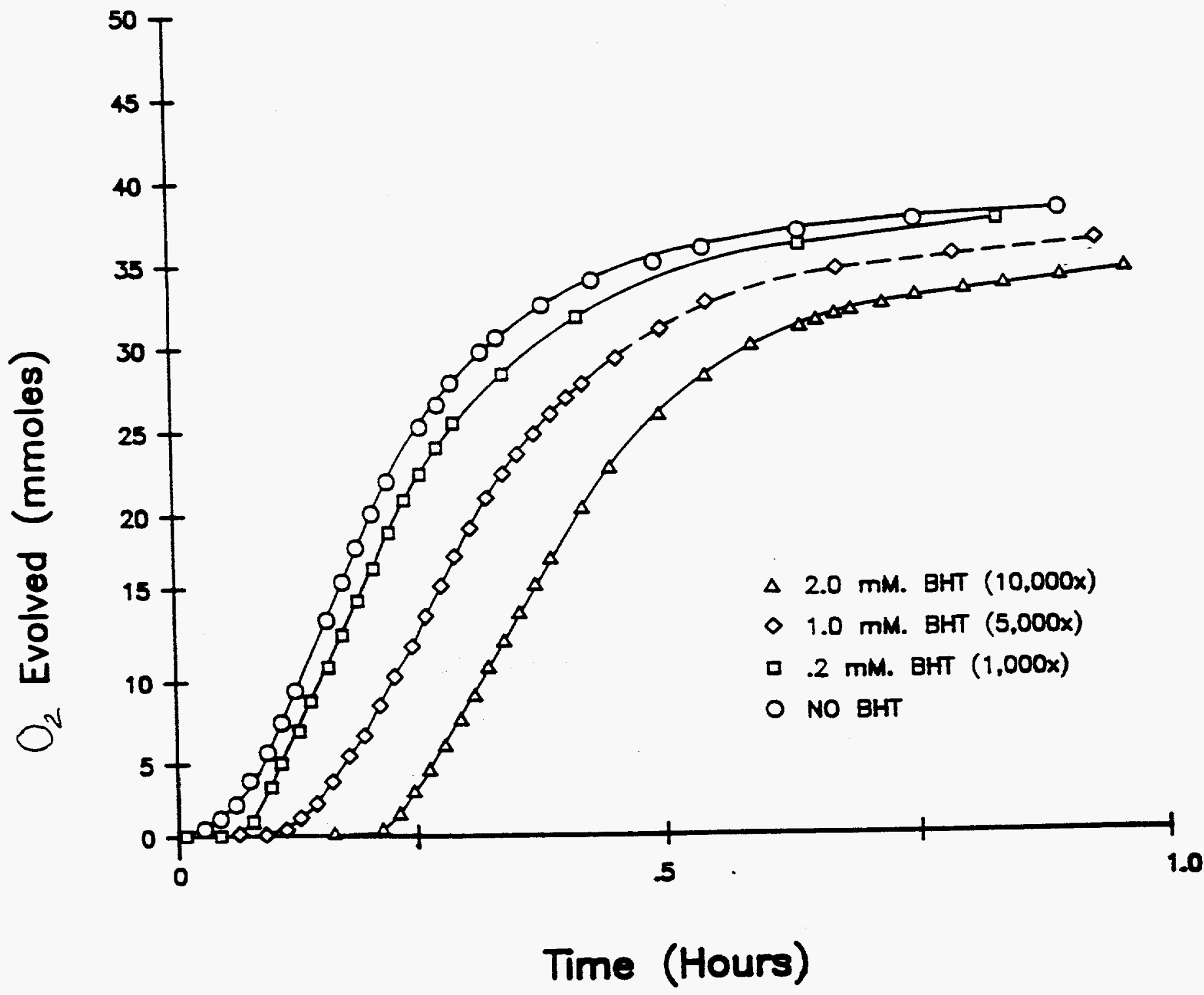




\section{Scheme 1}

Haber-Weiss decomposition of hydroperoxides using metalloporphyrins as catalysts (porphyrin ring omitted).

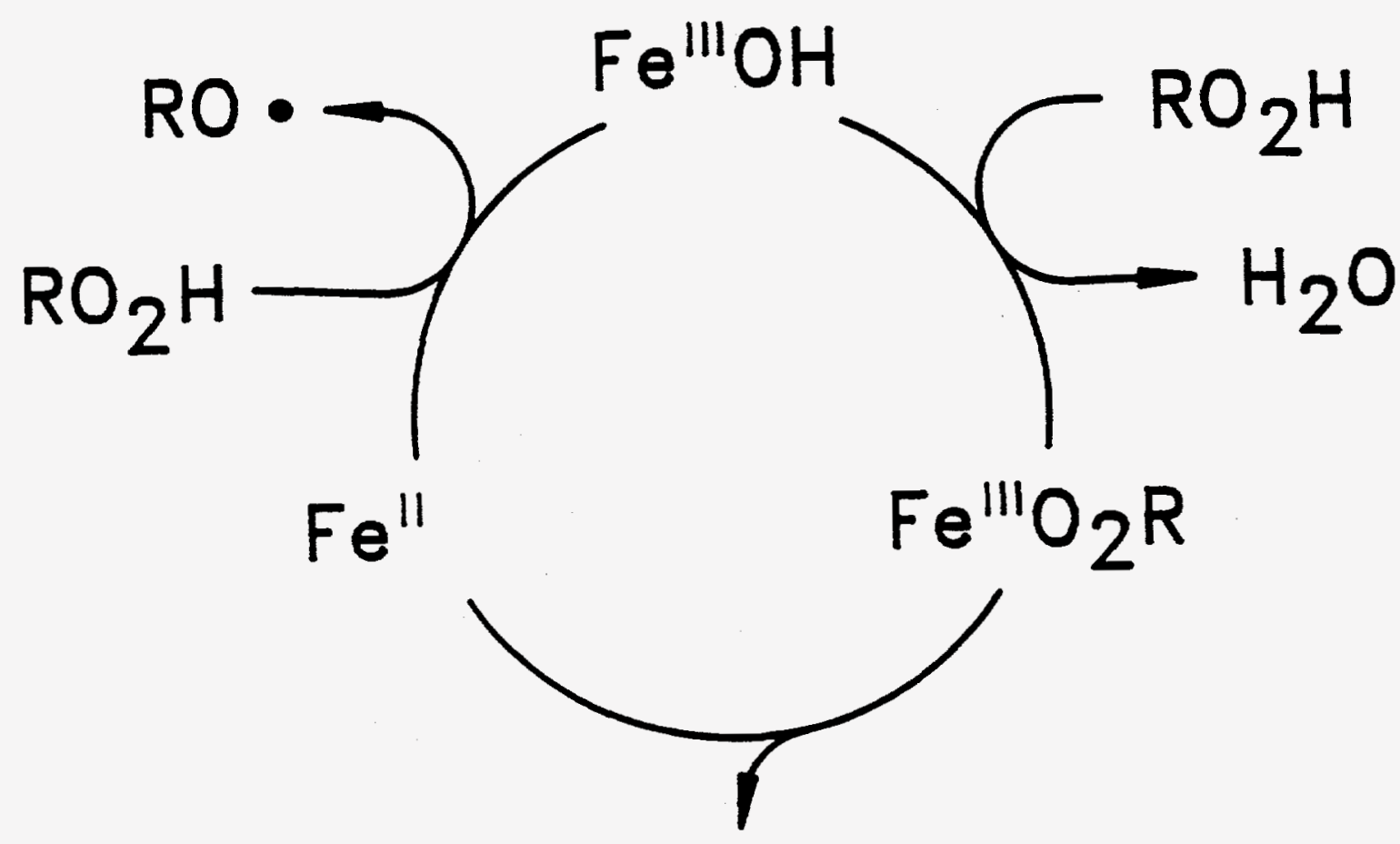

$\mathrm{RO}_{2} \cdot$

$2 \mathrm{RO}_{2} \mathrm{H} \longrightarrow \mathrm{RO}_{2} \cdot+\mathrm{RO} \bullet+\mathrm{H}_{2} \mathrm{O}$

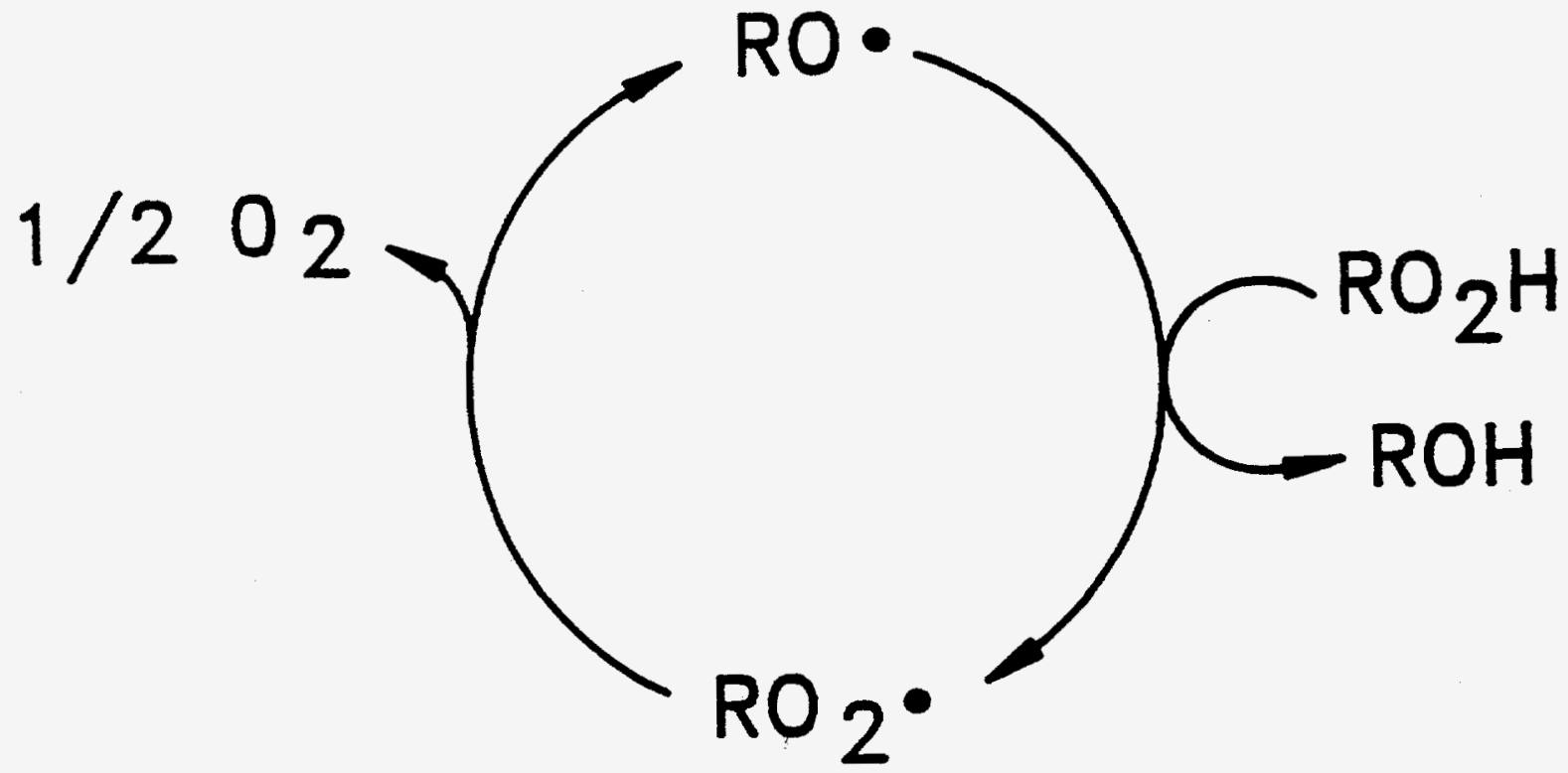




\section{Scheme 2}

Possible metal oxo pathways for decomposition of hydroperoxides using metalloporphyrin catalysts (porphyrin ring omitted).

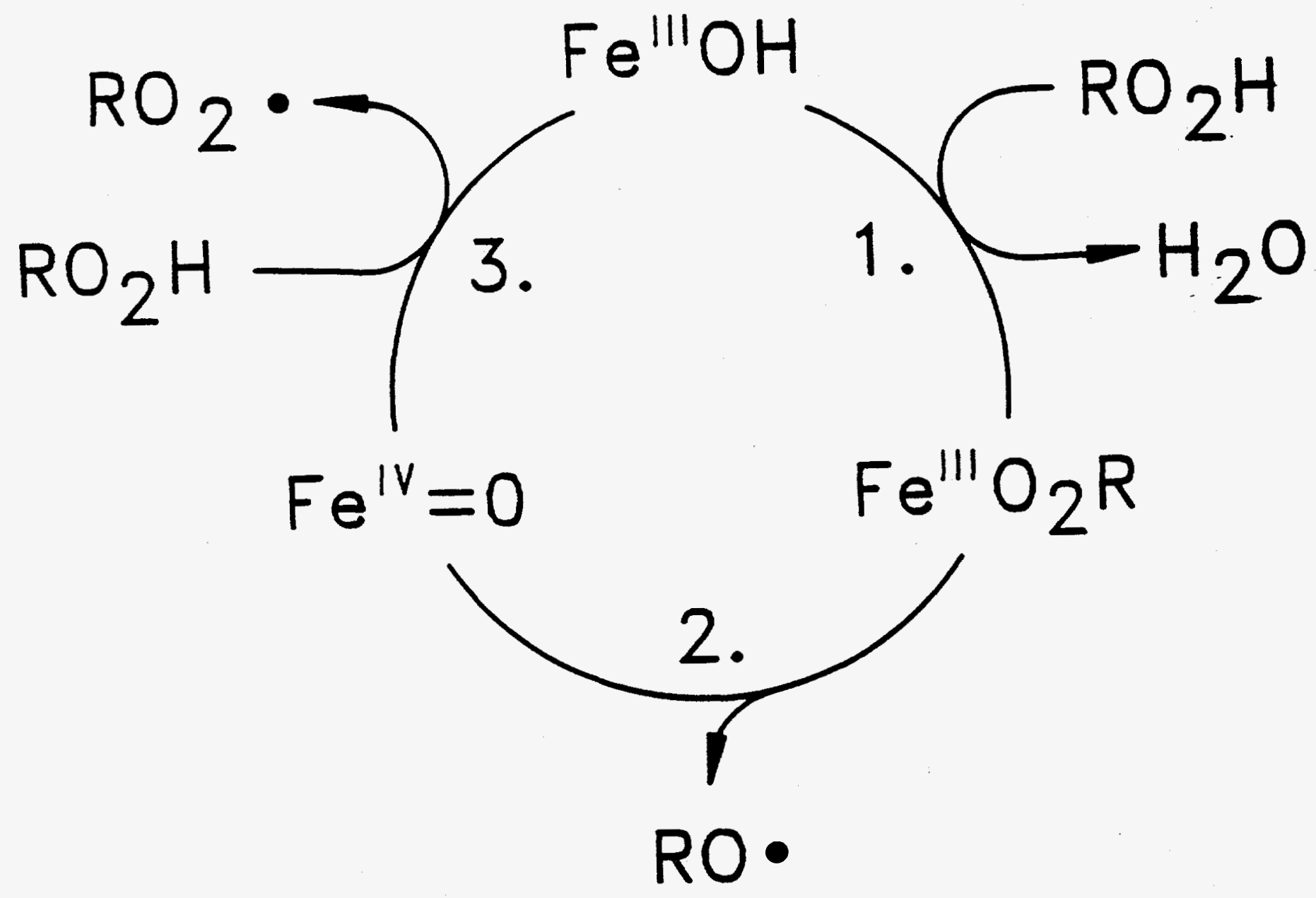




\subsection{HALOGENATED METALLOPORPHXRIN COMPLEXES AS CATALYSTS FOR SELECTIVE REACTIONS OF ACYCLIC ALKANES WITH MOLECULAR OXYGEN}

\subsection{ABSTRACT}

We have shown that halogenation of the porphyrin ring of a metalloporphyrin complex can convert a catalytically inactive material into an exceptionally active catalyst for the selective reaction of an alkane with molecular oxygen. The greater the degree of halogenation of the ring, the greater is the catalytic activity of the metal complex. The product profile, while characteristic of radical reactions, is sensitive to the nature of the metal center. Iron complexes are generally more active than those of cobalt, manganese or chromium. Activity of iron complexes is directly related to the $\mathrm{Fe}$ (III)/(II) reduction potential of the porphyrin complex. There is also a similar correlation between the $\mathrm{Fe}$ (III)/Fe(II) reduction potential and the rate at which iron haloporphyrin complexes decompose alkyl hydroperoxides. These iron perhaloporphyrin complexes are not only the most active known liquid phase alkane air-oxidation catalysts, they are also the most active hydroperoxide decomposition catalysts known to date. The nature of the products formed is dependent on the structure of the aliphatic substrate that is oxidized and can be rationalized by a catalytic pathway that very efficiently generates alkyl and alkoxy radicals at low temperatures. The relationship between the electrochemical properties of these complexes and the rates of alkane oxidation and hydroperoxide decomposition lends insight into possible mechanisms of catalytic activity.

\subsection{INTRODUCTION}

The selective reaction of an alkane with molecular oxygen to give an alcohol or carbonyl compound without extensive oxidative cleavage or concomitant combustion has considerable potential as an inexpensive means of producing valuable oxygenates. Selective oxidation of an alkane is usually hard to achieve thermally since under conditions necessary to promote facile autoxidation, alkane oxidations are often difficult to stop short of combustion (1). Metalloporphyrin complexes have long been known to catalyze oxidative hydroxylation of alkanes in a biomimetic manner, but these complexes have required either a) stoichiometric oxygen atom donors other than oxygen (2-10), b) sacrificial coreductants $(11-20)$, c) electrochemical reduction $(21,22)$, or d) photolytic assistance $(23,24)$.

Recent work by ourselves (25-34) and others (35-38) has shown that halogenated metalloporphyrins are efficient catalysts for the direct reaction of alkanes with oxygen to give alcohols and/or carbonyl compounds at unprecedented rates under very mild conditions. No coreductants or stoichiometric oxidants are required (25-38). Neither is it necessary to employ photochemical or electrochemical techniques in these oxidations. Because of the potential utility of a direct catalytic reaction of an alkane with molecular oxygen in a selective manner, we report the results of a study on the oxidation of a series of acyclic alkanes under mild conditions catalyzed by halogenated metalloporphyrins. 


\subsection{RESULTS AND DISCUSSION}

\section{Oxidations of Isobutane and Propane}

We have found that chromium, manganese and iron complexes of halogenated porphyrinato ligand systems, Figure 1, are active catalysts for the oxidation of unactivated acyclic alkanes having either secondary or tertiary carbon-hydrogen bonds. Primary $\mathrm{C}-\mathrm{H}$ bonds are quite resistant to oxidative attack in the presence of these catalysts. Table 1 shows the results of oxidations of isobutane at $80^{\circ} \mathrm{C}$ in benzene solutions of metalloporphyrin complexes using molecular oxygen as the oxidant. tert-Butyl alcohol is the predominant product of the reaction, being formed in yields in the neighborhood of $90 \%$. Several points are evident from the data presented in Table 1. Firstly, halogenation of the phenyl groups of tetraphenylporphyrinatometal complexes causes a large increase in their catalytic activity. Secondly, halogenation of the meso-phenyl groups on the macrocycle has a greater effect on the catalytic activity of iron complexes than on those of manganese and chromium. The effect on catalytic oxidation activity of meso-phenyl halogenation of metallotetraphenylporphyrin complexes is in the order $\mathrm{Fe}>\mathrm{Mn}>\mathrm{Cr}$. Thus, iron haloporphyrin complexes are the most active of those complexes that we have examined. The catalytic oxidation of isobutane using tetrakispentafluorophenylporphyrinatoiron hydroxo-,azido-,and chloro-complexes is shown in Figure 2. Rates are rapid and no induction periods are apparent. Table 2 shows the effect of the degree of halogenation on the catalytic oxidation activity of porphyrinatoiron chloro, azido oxo and hydroxo complexes. Figure 2 indicates the relative catalytic activities of the hydroxo-, azido-, and chloro forms of $\mathrm{Fe}\left(\mathrm{TPPF}_{20}\right) \mathrm{X}$. It is interesting to note that any induction period must be very short if it exists at all.

Table 3 shows the results of oxidations of isobutane at $60^{\circ} \mathrm{C}$ in the presence of perhaloporphyrinatometal azide complexes. At $80^{\circ} \mathrm{C}$, Table 1, complexes having axial chloride provide suitable catalysts, whereas for high activity at $60^{\circ} \mathrm{C}$ or lower it is desirable to use the azido- or hydroxo-forms of the catalyst. Combining the results in Tables 1,2 and 3, it can be seen that the greater the extent of halogenation of the porphyrin macrocycle, the more active is the metal complex for alkane oxidation. Table 4 shows the results of oxidations of propane at $125^{\circ} \mathrm{C}$ in the presence of perhaloporphyrinatometal azide complexes. Although higher temperatures are required to oxidize the secondary $\mathrm{C}-\mathrm{H}$ bond of propane than the tertiary $\mathrm{C}-\mathrm{H}$ bond of isobutane, the selectivity to a mixture of acetone and isopropyl alcohol is high and only small amounts of carbon oxides are formed.

Table 5 shows the results of oxidations with conventional cobalt oxidation catalysts. Prior to our work on the perhaloporphyrin complexes, the bispyridyliminoisoindoline, BPI, complexes were among the very most active alkane autoxidation catalysts known (39-40). A comparison of the results of Table 5 with those in Tables 1,2,3 and 4 indicates that perhaloporphyrin complexes are more active by over an order of magnitude than even the most active cobalt complexes currently available. 


\section{Effects of Electron Withdrawal From the Macrocycle}

As the number of halogen substituents around the periphery of the porphyrin macrocycle increases, the ease of reducing $\mathrm{Fe}$ (III) in the coordination sphere increases $(28-34,38)$. Figure 3 demonstrates a regular relationship between catalytic turnovers for both isobutane and propane oxidations in a set time period, and the $\mathrm{Fe}(\mathrm{III}) / \mathrm{Fe}$ (II) reduction potential of the catalyst used. Thus, as the degree of halogenation increases, the $\mathrm{Fe}$ (III)/Fe(II) reduction potential also increases and the catalytic activity improves. These iron complexes are soluble enough in aliphatic hydrocarbons to allow reactions to proceed in neat alkane solutions, Table 6 . When iron haloporphyrin complexes are dissolved in neat isobutane, oxidation to tert-butyl alcohol can be efficiently carried out under very mild conditions of temperature and pressure. These complexes smoothly catalyze isobutane oxidations at temperatures between room temperature and $100^{\circ} \mathrm{C}$, and oxygen partial pressures between one and five atmospheres. Reaction rate and catalyst life are unprecedented. We have achieved well over 20,000 turnovers (moles product(s) formed/mole catalyst used) in tert-butyl alcohol, TBA, production. Although catalyst decomposition is a problem at somewhat elevated temperatures $\left(>60^{\circ} \mathrm{C}\right)$, well over 10,000 catalytic turnovers have been observed in the room temperature oxidation of neat isobutane catalyzed by tetrakis(pentafluorophenyl $\beta$-octabromo)iron(III)hydroxide with no noticeable deferration or decay of the catalyst (by UV visible spectroscopy), Table 6 .

\section{Mechanism Considerations}

As one speculates on how oxidations catalyzed by electron deficient metalloporphyrins might proceed, it is instructive to consider the mechanism by which iron porphyrin systems are thought to catalyze $\mathrm{C}-\mathrm{H}$ bond oxidations in biological systems, Figure 4,(4-48). In enzymatic pathways electrons and protons are available to the heme system throughout the process. However, in our case, without coreductants (2-20), or photochemical or electrochemical assistance (21-24), monooxygenase activity of the type known to occur in vivo is not possible. Using the electrons of an oxygen-bridging iron catalyst system, Figure 5, one could envision a catalytic dioxygenase pathway. Steps a.,b.,c., and $\mathrm{f}$. in this hypothetical pathway have precedence in the literature (49-51). There remains the question as to whether a hypothetical Fe(IV)oxo intermediate would be effective for $\mathrm{C}-\mathrm{H}$ bond cleavage, Figure 5,d. Furthermore there is the strong possibility that radicals formed from $\mathrm{C}-\mathrm{H}$ bond homolysis, if it occurs, Figure 5,d., should be capable of diffusing into the reaction medium and initiating radical chain oxidation processes. Perhaps then, if radical escape is occurring, the pathway shown in Figure 5 ought to be considered as a form of living initiator of alkyl radicals as well as a possible catalytic precursor of alcohol by rebound mechanisms $(5,16)$, Figure 5 ,e.

The oxidation of organic compounds using metalloporphyrin complexes has been known for some time (52). Catalytic activity was generally poor, catalyst life was short and prior to our work (25-28) no alkane oxidation activity had been observed in unpromoted systems. Why then are the electron-deficient metalloporphyrins such good catalysts? One reason, of course, is the greater oxidative and thermal stability of the catalyst. Replacing hydrogens with halide substituents reduces the number of carbon hydrogen bonds that can undergo oxidative attack. A perhaloporphyrin complex has no carbon hydrogen bonds which might undergo cleavage. In addition, as electron withdrawing 
halo groups are introduced into the macrocyclic ring of the catalyst, the ligand itself becomes more stable to reduction by the central metal atom. It is well known that the porphyrinato ligand in high oxidation state porphyrinatoiron oxo complexes exists as the radical cation due to electron transfer from the ligand to the iron center $(35,53)$. Thus, there may be a greater tencency for the active catalyst to behave as an oxo centered radical, $(\mathrm{P}) \mathrm{Fe}-\mathrm{O}^{\circ}$, than a ferryl species, $\left(\mathrm{P}^{+*} \mathrm{Fe}=0\right.$, with increased electron withdrawal from the macrocycle (35).

Our work has shown that not only are electron-deficient metalloporphyrin complexes more robust, but initial rates of oxidation are far faster than observed when non-halogenated porphyrin complexes are used as catalysts. In fact, using halogenated metalloporphyrin catalysts, oxidations occur at temperatures so low that most metalloporphyrin complexes are completely inactive, Tables 1-4. Increased reaction rate can be rationalized as a result of increased $\mathrm{Fe}(\mathrm{III}) / \mathrm{Fe}$ (II) reduction potential due to electron withdrawal from the metal center by the electron deficient ligand system. Thus, in most metalloporphyrin-catalyzed oxidations, the pool of iron exists largely or exclusively as iron(III). Initial formation of Fe(II) is disfavored, and the process represented in Figure 5,e does not occur readily. Most of the iron is rapidly converted to the $\mu$-oxo dimer, Figure $5, \mathrm{f}$, which is usually inactive $(54,55)$. We have shown that $\mu$-oxo dimers are active catalysts in the perhaloporphyrin series, Tables 1,2 and that both iron(III) $\mu$-oxo dimer and $F e($ III) species co-exist under reaction conditions (30). Therefore, although we by no means have established the existance of the scheme depicted in Figure 5, electron deficient porphyrins might indeed possess the necessary properties to behave in this manner, while more electron-rich porphyrin complexes do not.

\section{Radical Routes and Hydroperoxide Intermediates}

The homolysis of $\mathrm{C}-\mathrm{H}$ bonds in biological systems occurs in a cage which encourages rebound to produce alcohols. Radicals do not readily escape into an aqueous medium in vivo as they might be expected to do of. Figure 5,d, when an electron-deficient metalloporphyrin catalyst is used in organic media. If a substantial amount of the reaction proceeds through a radical pathway, then a free radical inhibitor should quench the reaction. We have shown that as little as one mole of di-tert-butyl-p-cresol per mole of catalyst can substantially quench the oxidation reaction. Furthermore, if radical pathways are important, then peroxidic and hydroperoxidic intermediates formed by reactions of alkyl radicals with dioxygen should be involved, eq. 1,2(56-58).

$$
\begin{aligned}
& \mathrm{R}^{\bullet}+\mathrm{O}_{2} \longrightarrow \mathrm{RO}_{2}^{\bullet} \\
& \mathrm{RO}_{2}^{\bullet}+\mathrm{RH} \longrightarrow \mathrm{RO}_{2} \mathrm{H}+\mathrm{R}^{\bullet}
\end{aligned}
$$

It was interesting, therefore, to find (59) that halogenated porphyrins are highly active catalysts for the decomposition of alkyl hydroperoxides to form alcohols in a selective manner, eq. 3, Table 7, Fig 6. In addition, the more highly halogenated the porphryin macrocycle, the greater the activity of the

$$
\mathrm{RO}_{2} \mathrm{H} \longrightarrow \mathrm{ROH}+\mathrm{O}_{2}
$$


metalloporphyrin catalyst for this reaction. Thus, as shown in Figure 3 and Table 8, the greater the $\mathrm{Fe}(\mathrm{III}) / \mathrm{Fe}$ (II) reduction potential, the greater the rate of alkane oxidation and the greater the rate of hydroperoxide decomposition. We have proposed (59) that a modified Haber-Weiss cycle, Figure 7, creates the alkoxy and alkylperoxy radicals that lead to the stable products of decomposition: $\mathrm{ROH}$ and oxygen. A kinetic analysis of this data (60) by Labinger has shown that such a pathway, with the slow step being reduction of $\mathrm{Fe}(\mathrm{III})$ to $\mathrm{Fe}(\mathrm{II})$ by alkyl hydroperoxide could account for rapid TBA formation during autoxidation when electron deficient porphyrins with high $\mathrm{Fe}(\mathrm{III}) / \mathrm{Fe}$ (II) reduction potentials are used as catalysts. In fact, Labinger's analysis suggests that the majority of the isobutane oxidation reaction may proceed through a metalloporphyrin-assisted radical chain rather than a purely catalytic reaction mechanism.

\section{Alkane Structure and Product Profile}

The high selectivity observed for the oxidations of isobutane and propane shown above is not characteristic of radical reactions. The reason for this may be the low temperatures at which it is possible to conduct reactions using the highly active metallohaloporphyrin catalysts. A structural feature of these two substrates that enhances selectivity is that all carbon-carbon bonds are carbon-methyl bonds. This results in a low likelihood of $\mathrm{C}-\mathrm{C}$ bond cleavage ( $\beta$-scission)(61) from a possible alkoxy radical intermediate since $\beta$-scission requires formation of an unstable methyl radical (eq. 4). Thus, few carbon-carbon bond cleavage or combustion products are formed in the mild

$$
\mathrm{CH}_{3}-\mathrm{C}-\mathrm{O} \longrightarrow \mathrm{CH}_{3} \bullet+\mathrm{C}=\mathrm{O}
$$

oxidations of isobutane and propane. If, on the other hand, one conducts reactions of $\underline{n}$-butane with oxygen in the presence of perhaloporphyrin complexes, significant oxidative cleavage results, forming by-products such as acetic acid and acetaldehyde, Table 9 . It is of interest to note that selectivity to 2-butanone is enhanced by chromium complexes over those of iron. The azido form of the chromium complex is most effective for generating ketones via alkane oxidations. Oxidations carried out in the presence of this catalyst are very rapid and exhibit the characteristic sigmoid kinetic curve of autoxidations including the presence of a substantial induction period, Figure 8.

Table 10 details the product profiles for the oxidations of a series of branched alkanes with $3^{\circ} \mathrm{C}-\mathrm{H}$ bonds and Figure 9 summarizes the dependence of product profile on substrate structure for oxidations catalyzed by tetrakis(pentafluorophenyl $\beta$-octabromo)porphyrinatoiron(III)hydroxide. Those hydrocarbons having tertiary $\mathrm{C}-\mathrm{H}$ bonds react at temperatures of $60^{\circ} \mathrm{C}$ or less while those having secondary but no tertiary $\mathrm{C}-\mathrm{H}$ bonds require more elevated temperatures and primary $\mathrm{C}-\mathrm{H}$ bonds have very low reactivity at any of the temperatures used. The relative $\mathrm{C}-\mathrm{H}$ bond reactivity pattern: $3^{\circ}>2^{\circ}>1^{\circ}$ is consistent with that of radical initiated oxidation pathways $(1 \mathrm{~b}, 49,50,62)$. 
The ratio of selective oxidation to cleavage products in the case of propane or isobutane oxidations is $\sim 9 / 1$. On the other hand, the ratio of four or five-carbon oxidation products to cleavage products is less than $2 / 1$ in oxidations of n-butane and 2-methylbutane respectively, and when 3-methylpentane is oxidized, the oxygenation/cleavage ratio drops below 1. It is interesting in this regard that decomposition of tert-amyl hydroperoxide, TAHP, leads to a far greater extent of carbon-carbon bond cleavage than decomposition of tert-butyl hydroperoxide, Table 11. Furthermore, the ratio of tert-amyl alcohol to acetone produced during catalyzed TAHP decomposition in benzene at room temperature is very similar to the ratio of these products formed via 2-methyl butane oxidation, Table 10. This observation is consistent with the common intermediacy of the 2-methyl-but-2-oxy radical in both cases, eq. 5 .

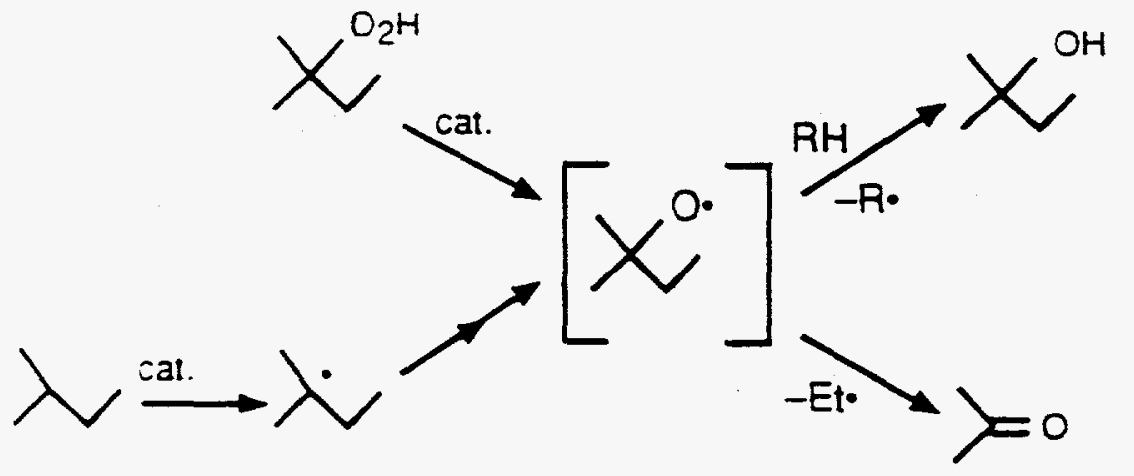

When the symmetrical hydrocarbon, 2,3-dimethylbutane is used, a substrate having two easily abstractable $3^{\circ} \mathrm{C}-\mathrm{H}$ bonds and an isopropyl group adjacent to either internal carbon atoms, cleavage predominates over six-carbon oxidation products by a wide margin.

Thus, by varying the structure of the alkane substrate we are able to move from selective oxygenations that retain the intact carbon skeleton and give only $10 \%$ carbon-carbon bond cleavage or less, to greater than $90 \% \mathrm{C}-\mathrm{C}$ bond cleavage reactions in these alkane oxidations. The direction of the effect of alkane structure on carbon-carbon bond scission is consistant with the intermediacy of alkoxy radicals as significant reaction intermediates $(61,62)$

Iron haloporphyrin catalyzed oxidation of 2,2,3-trimethylbutane, eq.6,7 gives rise to equimolar amounts of acetone and products of the very stable tert-butyl radical. The hypothetical alkoxy radical in this case would cleave nearly exclusively giving very little $C_{7}$ oxygenate. 

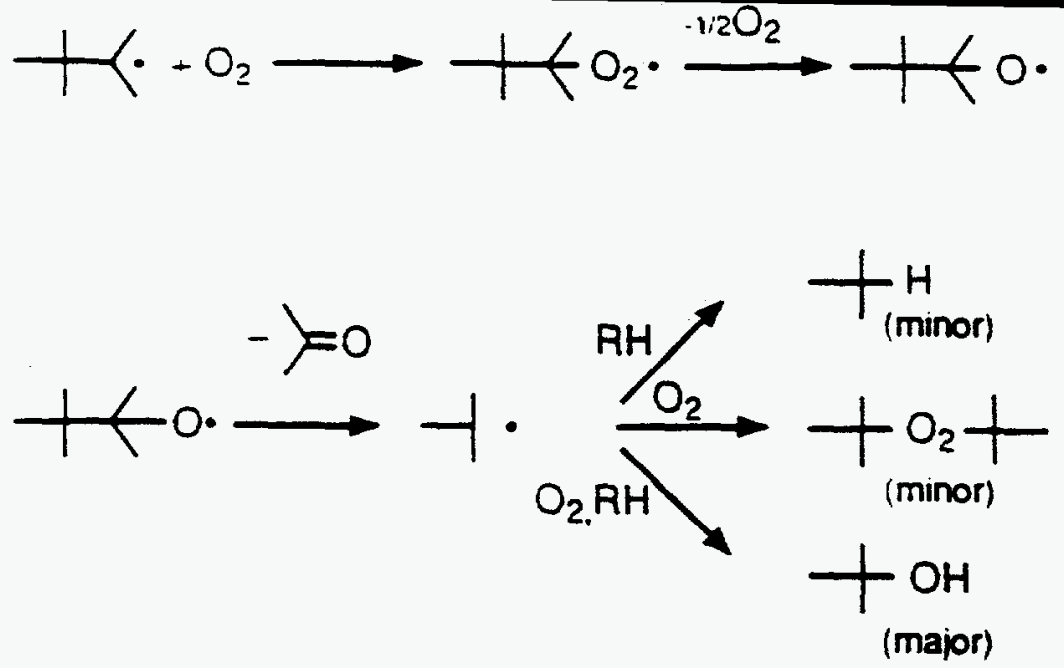

Greene and coworkers (62) have demonstrated that the ratio of rates of alkyl cleavage from alkoxy radicals vs. $\mathrm{H}$ abstraction from cyclohexane at $40^{\circ} \mathrm{C}$ increases dramatically from methyl to ethyl to isopropyl. In Table 12 we compare the $\%$ cleavage products formed during catalytic oxidations at 60 and $80^{\circ} \mathrm{C}$ with this ratio and find qualitative similarities. Differences arise since $\mathrm{H}$-abstraction in the oxidation cases would be largely from $3^{\circ} \mathrm{C}-\mathrm{H}$ rather than from $2^{\circ} \mathrm{C}-\mathrm{H}$ bonds. Nonetheless, there is clear directional similarity between the extent of intramolecular $\mathrm{C}-\mathrm{C}$ bond cleavage reactions from alkoxy radicals and the selectivity of metalloporphyrin catalyzed oxidations that we have conducted.

\subsection{CONCLUSIONS}

Halogenated metalloporphyrin complexes are extremely active catalysts for the selective oxidation of alkanes and the decomposition of alkylhydroperoxides to alcohols. Perhalotetraphenylporphyrinatoiron complexes are the most active catalysts known for both of these reactions giving rates an order of magnitude greater than any previously reported. The low temperatures at which these oxidations can be conducted result in some unique and useful selectivities.

Although biomimetic chemistry may be involved in initiation of radical chains, Figure $5 \mathrm{~d}$, and indeed in a degree of dioxygenase-like catalysis, Figure 5e, decomposition of peroxidic intermediates by iron perhaloporphyrin catalysts is also of great importance. The bulk of the available evidence indicates that radical chain processes are occurring. Figure 10 indicates a possible scheme in which the metal complexes initiate formation of radicals, 1, that generate hydroperoxides, 2 , which are catalytically converted to radicals, 3 , that produce stable oxidation products, 4 . If this is so, then the majority of the alcohol produced in the oxidation of isobutane, for example, would arise by hydrogen abstraction, 5 , rather than by biomimetic rebound fig 4,e. It seems likely that all of these processes are proceeding in these oxidations and further work will sort out the relative importance of each.

\subsection{EXPERIMENTAL SECTION}

General. 'HNMR (300MHz Bruker MSL), ${ }^{19} \mathrm{FNMR}$ (300 MHz Nicolet NT-300), IR spectra (Mattson R/S FTIR), absorption spectra (HP 8452A) and ion cycloctron resonance mass spectra (Nicolet FTMS) were collected routinely. Fast atom bombardment mass spectra were collected by M-Scan, Inc. (West Chester, PA.) Laser desorption mass spectra were collected at the University of Delaware on a Nicolet LD-FTMS 2000 instrument. 
Solvents. All solvents used were HPLC grade from Aldrich and were used as purchased unless further purification is noted. $\mathrm{CHCl}_{3}$ used contained $0.75 \%$ ethanol unless it is noted that ethanol-free $\mathrm{CHCl}_{3}$ was used.

Reagents. All non-porphyrin reagents were purchased from Aldrich unless noted and were certified ACS reagent grade or better. FeTPPCl, MnTPP(OAc), and (FeTPP) ${ }_{2} \mathrm{O}$ were purchased from Strem Chemicals. FeTPPF 20 Cl and $\mathrm{H}_{2} \mathrm{TPPF}_{20}$ were purchased from Aldrich. $\mathrm{Fe}\left(\mathrm{TPPCl}_{8} \beta-\mathrm{Br}_{4}\right) \mathrm{Cl}$ was purchased from Midcentury Chemicals. CrTPPCl(63), MnTPPN ${ }_{3}$ and $\mathrm{CrTPPN}_{3}(64), \mathrm{FeTPPN}_{3}$ (65), $\left[\mathrm{Fe}\left(\mathrm{TPP} \beta-\mathrm{Br}_{4}\right)\right]_{2} \mathrm{O}(66), \mathrm{Fe}\left(\mathrm{TPP} \beta-\mathrm{Br}_{4}\right) \mathrm{Cl}(67), \mathrm{Fe}\left(\mathrm{TPPCl}_{8}\right) \mathrm{Cl}$ and $\mathrm{Fe}\left(\mathrm{TPPCl}_{8}\right) \mathrm{OH}$ (68), $\mathrm{Fe}\left(\mathrm{TPPF}_{20}\right) \mathrm{OH}$ and $\left(\mathrm{FeTPPF}_{20}\right)_{2} \mathrm{O}(69), \mathrm{Cr}\left(\mathrm{TPPF}_{20}\right) \mathrm{Cl}(70), \mathrm{Mn}\left(\mathrm{TPPF}_{20}\right) \mathrm{OAc}$ (71), $\mathrm{Co}(\mathrm{BPI}) \mathrm{OAc}$ (72), and $\mathrm{Co}(\mathrm{BPI})\left(\mathrm{O}_{2} \mathrm{Bu}^{\mathrm{t}}\right)(40)$ were made by the referenced methods.

Oxidation Methods. Oxidations were carried out either in $60 \mathrm{cc}$ Fisher-Porter aerosol tubes or in 300-500 ml batch autoclaves as indicated in the Tables. Reactions run in Fisher-Porter tubes equipped with a baffled magnetic stirrer, were carried out at 100 psig total pressure at the temperatures indicated in the Tables. The catalyst was dissolved in either the neat alkane or in a benzene solution to which the alkane was added. The Fisher-Porter tube was attached to a manifold for adding and releasing gases. Oxygen was admitted to the tube and the tube was then submerged in a constant temperature bath at reaction temperature. The reaction mixture was magnetically stirred for the amount of time indicated in the Table. Gas uptake was measured by a pressure gauge and oxygen was replenished as it was consumed. At the time indicated in the Table, the tube was removed from the bath, cooled to $0^{\circ} \mathrm{C}$ and the gas slowly vented. The liquid product was weighed and analyzed by standardized glpc. Caution: Many of the reactions conducted in this work were done within the explosion range. Reactions were performed in a properly barricaded laboratory area for safety.

Propane oxidations were routinely performed in a glass-lined $300 \mathrm{ml}$ stainless steel autoclave fitted with thermocouple and magnedrive stirrer. All internals: cooling coils, thermocouple, and stirrer blades and shaft were coated with Teflon. A solution of the catalyst in benzene was added to the glass liner, the autoclave secured and liquid propane was pumped into the reactor. Air was added to a total pressure of $1500 \mathrm{psig}$ and the reactor heated to reaction temperature. After the reaction time indicated in the Tables, the reactor was cooled to room temperature, gases slowly vented into a gas bag for glpc analysis, and liquid product was analyzed by standardized glpc.

Hydroperoxide decompositions. A solution of the catalyst in p-xylene (internal standard) was quickly added to a rapidly stirring solution of tert-butyl hydroperoxide in benzene at room temperature. Oxygen evolution was measured manometrically and liquid products were analyzed periodically by standardized gas chromatography. [CAUTION - Reactions should be run in dilute solutions of catalyst and/or hydroperoxide to avoid exotherms and dangerously fast gas evolution.]

Benzene, (99.99\%, Aldrich), tert-butyl alcohol, (99.6\%, Aldrich), and p-xylene, $(99+\%$, Aldrich) were used as purchased. tert-Butyl hydroperoxide ( $90 \%, 5 \%$ water, $5 \%$ tert-butyl alcohol) was used as purchased from Aldrich. 
$\mathrm{Mn}\left(\mathrm{TPPF}_{20}\right) \mathrm{N}_{3} .0 .4 \mathrm{~g}$ of $\mathrm{Mn}\left(\mathrm{TPPF}_{20}\right) \mathrm{OAc}$ is dissolved in $40 \mathrm{ml}$ of $\mathrm{CHCl}_{3} .10 \mathrm{ml}$ of saturated aqueous $\mathrm{NaN}_{3}$ solution is stirred vigorously with the $\mathrm{CHCl}_{3}$ solution overnight. The water layer is separated and $10 \mathrm{ml}$ of fresh $\mathrm{NaN}_{3}$ solution is added and stirred again overnight. The water layer is removed and the $\mathrm{CHCl}_{3}$ is washed 3 times with $20 \mathrm{ml}$ of $\mathrm{H}_{2} \mathrm{O}$. The $\mathrm{CHCl}_{3}$ solution is dried over $\mathrm{Na}_{2} \mathrm{SO}_{4}$ then filtered and rotovapped to dryness. This material is washed with copious amounts of $\mathrm{H}_{2} \mathrm{O}$ then dried overnight in a vacuum over $\mathrm{P}_{2} \mathrm{O}_{5}$ at $60^{\circ}$. Yield $0.36 \mathrm{~g}$. IR shows a N-N stretch at 2030 $\mathrm{cm}^{-1}$.

$\operatorname{Cr}\left(\mathrm{TPPF}_{20}\right) \mathrm{N}_{3} .400 \mathrm{mg}$ of $\mathrm{Cr}\left(\mathrm{TPPF}_{20}\right) \mathrm{Cl}$ is dissolved in $100 \mathrm{ml}$ of acetone. $4.0 \mathrm{~g}$ of $\mathrm{NaN}_{3}$ is added and the mixture is stirred for $48 \mathrm{hrs}$. at room temperature. After the solvent is removed and the solid product is washed with $\mathrm{H}_{2} \mathrm{O}$ and dried, a yield of $350 \mathrm{mg}$ of $\mathrm{Cr}\left(\mathrm{TPPF}_{20}\right) \mathrm{N}_{3}$ is obtained. $\mathrm{IR}(\mathrm{KBr})$ shows a N-N stretch at $2053 \mathrm{~cm}^{-1}$.

$\mathrm{Fe}\left(\mathrm{TPPF}_{20}\right) \mathrm{N}_{3} .0 .5 \mathrm{~g}$ of Fe(TPPF 20$) \mathrm{Cl}$ is dissolved in $100 \mathrm{ml}$ of acetone. To this is added $5.0 \mathrm{~g}$ of $\mathrm{NaN}_{3}$. Most of the $\mathrm{NaN}_{3}$ and all of the $\mathrm{Fe}\left(\mathrm{TPPF}_{20}\right) \mathrm{Cl}$ is dissolved. This mixture is stirred at room temperature for $24 \mathrm{hrs}$. then filtered and rotovapped to dryness. The material is washed with large amounts of $\mathrm{H}_{2} \mathrm{O}$ then vacuum dried overnight at $60^{\circ}$. Yield $0.4 \mathrm{~g}$. IR shows (N-N) stretch of azide at $2053 \mathrm{~cm}^{-1}$. Large crystals were grown by recrystallization in $5 \%$ hexane in chloroform.

$\mathrm{Fe}\left(\mathrm{TPPF}_{20} \mathrm{\beta}-\mathrm{Br}_{\mathbf{8}}\right) \mathrm{Cl}$. Under $\mathrm{N}_{2}, 0.500 \mathrm{~g}$ of $\mathrm{Fe}\left(\mathrm{TPPF}_{20}\right) \mathrm{Cl}$ is dissolved in $300 \mathrm{ml}$ of degassed $\mathrm{CCl}_{4}$ which was freshly distilled (over $\mathrm{P}_{2} \mathrm{O}_{5}$ ). $100 \mathrm{ml}$ of $6 \mathrm{MBr}_{2} / \mathrm{CCl}_{4}$ is added quickly to this solution while at reflux. After 18 hours the Soret absorption has moved from $418 \mathrm{~nm}$ to $429 \mathrm{~nm}$. $50 \mathrm{ml}$ more of $6 \mathrm{MBr}_{2}$ in $\mathrm{CCl}_{4}$ is added at this point. After several more hours of reflux the Soret absorption remains at $429 \mathrm{~nm}$. The solution is cooled, filtered and washed three times with $\mathrm{H}_{2} \mathrm{O}$ then evaporated to dryness. The greenish-brown material is chromatographed on neutral alumina eluting with $\mathrm{CHCl}_{3}$. The first green band is collected, treated with dilute $\mathrm{HCl}$ and evaporated to dryness. After drying in vacuo at $110^{\circ} \mathrm{C}, 590 \mathrm{mg}$ of the $\mathrm{Fe}\left(\mathrm{TPPF}_{20} \beta-\mathrm{Br}_{8}\right) \mathrm{Cl}$ is obtained. $\mathrm{FAB} / \mathrm{MS}: \mathrm{M}^{+}-\mathrm{Cl} 1659$.

$\mathrm{Fe}\left(\mathrm{TPPF}_{20} \beta-\mathrm{Br}_{8}\right) \mathrm{N}_{3} .300 \mathrm{mg}$ of $\left.\mathrm{Fe}\left(\mathrm{TPPF}_{20} \beta-\mathrm{Br}\right)_{8}\right) \mathrm{Cl}$ is dissolved in $300 \mathrm{ml}$ of methanol and 200 $\mathrm{ml}$ of acetone. To this solution is added $3.5 \mathrm{~g}$ of $\mathrm{NaN}_{3}$ then 10 drops of glacial acetic acid. The solution is stirred for $72 \mathrm{hr}$. then filtered and the solvent is removed by rotary evaporation. The dry material is washed with water and dried in vacuo at room temperature. Yield is quantitative. $\mathrm{IR}(\mathrm{KBr}): v_{\mathrm{N}-\mathrm{N}} 2049 \mathrm{~cm}^{-1}$.

$\operatorname{Cr}\left(\mathrm{TPPF}_{20} \beta-\mathrm{Br}_{8}\right) \mathrm{Cl}$. Under $\mathrm{N}_{2}, 0.700 \mathrm{~g}$. of $\operatorname{Cr}\left(\mathrm{TPPF}_{20}\right) \mathrm{Cl}$ is dissolved in $600 \mathrm{ml}$. of freshly distilled (over $\mathrm{P}_{2} \mathrm{O}_{5}$ ) $\mathrm{CCl}_{4}$. The solution is heated to $40^{\circ} \mathrm{C}$ and $150 \mathrm{ml}$. of $\mathrm{Br}_{2}$ is added dropwise with stirring. The Soret absorption of the unreacted chromium porphyrin at $436 \mathrm{~nm}$ shifts to $481 \mathrm{~nm}$ after $22 \mathrm{hrs}$. reaction time. At this point, the solution is cooled, filtered and washed three times with water, then evaporated to dryness. The green material is redissolved in a minimum of $\mathrm{CHCl}_{3}$ and chromatographed on neutral alumina. The solution is washed with $1 \mathrm{~N} \mathrm{HCl}$ then evaporated to dryness. Yield $350 \mathrm{mg}$. UV/VIS (toluene), 400, 483, $599 \mathrm{~nm}$. 
$\operatorname{Cr}\left(\mathrm{TPPF}_{2} \beta-\mathrm{Br}_{8}\right) \mathrm{N}_{3} . \quad 300 \mathrm{mg}$ of $\mathrm{Cr}\left(\mathrm{TPPF}_{20} \beta-\mathrm{Br}_{8}\right) \mathrm{Cl}$ is dissolved in $300 \mathrm{ml}$ of methanol and 200 $\mathrm{ml}$ of acetone. To this solution is added $3.5 \mathrm{~g}$ of $\mathrm{NaN}_{3}$ then stirred for $48 \mathrm{hr}$. After filtering, the solvent is removed by rotary evaporator and the solids washed with water and dried in vacuo at room temperature. Yield: $260 \mathrm{mg}$. $\mathbb{I R}(\mathrm{KBr}): v_{\mathrm{N}-\mathrm{N}} 2050 \mathrm{~cm}^{-1}$.

$\mathrm{Mn}\left(\mathrm{TPPF}_{20} \beta-\mathrm{Br} \mathrm{r}_{8}\right) \mathrm{Cl} . \mathrm{Mn}\left(\mathrm{TPPF}_{20}\right) \mathrm{Cl}, .0 .400 \mathrm{~g}$., is dissolved in $300 \mathrm{ml}$ of freshly distilled (over $\left.\mathrm{P}_{2} \mathrm{O}_{5}\right) \mathrm{CCl}_{4}$ under $\mathrm{N}_{2}$. The solution is warmed and $125 \mathrm{ml}$ of $\mathrm{Br}_{2}$ is added dropwise with stirring. The reaction is continued until a sample shows a Soret of greater than $450 \mathrm{~nm}$. After washing three times with water the solvent is evaporated. The material is dissolved in a minimum of $\mathrm{CHCl}_{3}$ and chromatographed on neutral alumina. The $\mathrm{CHCl}_{3}$ solution is washed with $1 \mathrm{~N} \mathrm{HCl}$ then evaporated to dryness. Yield $320 \mathrm{mg}$. UV/VIS (Toluene): 399, 452, 495, 603.

$\mathrm{Mn}\left(\mathrm{TPPF}_{20} \beta-\mathrm{Br}_{8}\right) \mathrm{N}_{3} .150 \mathrm{mg}$ of $\mathrm{Mn}\left(\mathrm{TPPF}_{20} \beta-\mathrm{Br}_{8}\right) \mathrm{Cl}$ is dissolved in $150 \mathrm{ml}$ of methanol and $100 \mathrm{ml}$ of acetone. $1.5 \mathrm{~g}$ of $\mathrm{NaN}_{3}$ is added and the mixture stirred for 48 hours. After filtration and removal of the solvent, the solids are washed with water. $130 \mathrm{mg}$ of product is recovered $\mathbb{R}(\mathrm{KBr})$ : $v_{N-N} 2037 \mathrm{~cm}^{-1}$.

$\mathbf{H}_{2} \mathrm{TPPF}_{20} \beta-\mathrm{Cl}_{8}$. The preparation of this porphyrin is accomplished by the chlorination of $\mathrm{ZnTPPF}_{20}$ with $\mathrm{Cl}_{2}$ and in-situ removal of $\mathrm{Zn}$ with $\mathrm{HCl}$.

A three-necked flash fitted with reflux condenser and $\mathrm{N}_{2}$ inlet port is charged with $1.0 \mathrm{~g}(0.96$ mmol) of $\mathrm{Zn}\left(\mathrm{TPPF}_{20}\right)$ and $500 \mathrm{ml} \mathrm{CCl}$ degassed previously with $\mathrm{N}_{2}$. The $\mathrm{N}_{2}$ flow is terminated and $\mathrm{Cl}_{2}$ is bubbled through the solution slowly for $4 \mathrm{~min}$. while the solution is refluxed. After $4 \mathrm{~min}$. the $\mathrm{Cl}_{2}$ flow is stopped and $\mathrm{N}_{2}$ is used above the condenser to blanket the apparatus. Ater $1 \mathrm{hr}$. of reflux an aliquot is removed and the Soret absorption is checked. The reaction is complete when the Soret reaches $448 \mathrm{~nm}$. If the reaction is incomplete then a second $4 \mathrm{~min}$. $\mathrm{Cl}_{2}$ addition is performed with subsequent $1 \mathrm{hr}$. of additional reflux period. The solution is allowed to cool to $60^{\circ}$ then $\mathrm{HCl}$ gas is bubbled through the hot solution for $2 \mathrm{~min}$. and stirred for $15 \mathrm{~min}$. more. TLC (silica gel, $\mathrm{CH}_{2} \mathrm{Cl}_{2}$ ) is used to check that the $\mathrm{Zn}$ removal is complete. The green solution is cooled then washed with $200 \mathrm{ml}$ of $5 \% \mathrm{NaHCO}_{3}$ then the solvent is removed by rotary evaporation. Chromatography on neutral alumina eluting with $\mathrm{CHCl}_{3}$ gave $1.28 \mathrm{~g}$ (71\% yield). LDMS: $\mathrm{M}^{+}=1250$. ${ }^{19} \mathrm{FNMR}\left(\mathrm{CDCl}_{3}, 282.307\right.$ $\mathrm{MHz}$ referenced to $\left.\mathrm{C}_{6} \mathrm{D}_{5} \mathrm{~F}\right)$ :- $136.88(\mathrm{~m}, 8 \mathrm{~F}, \mathrm{Q}-\mathrm{ArF}),-148.34$ ( $\left.\mathrm{t}, 4 \mathrm{~F}, \mathrm{p}-\mathrm{ArF}\right),-160.50(\mathrm{~m}, 8 \mathrm{~F}, \mathrm{~m}-\mathrm{ArF}$ ).

$\mathrm{Fe}\left(\mathrm{TPPF}_{20} \beta-\mathrm{Cl}_{8}\right) \mathrm{Cl}$. In a $250 \mathrm{ml}$ flask is charged $750 \mathrm{mg}$ of $\mathrm{H}_{2} \mathrm{TPPF}_{20} \beta-\mathrm{Cl}_{8}, 200 \mathrm{ml}$ of DMF and $10 \mathrm{ml}$ of acetic acid. After heating to $160^{\circ}$ under $\mathrm{N}_{2}, 240 \mathrm{mg}$ of powdered $\mathrm{FeCl}_{2} .4 \mathrm{H}_{2} \mathrm{O}$ is added with stirring. After 10-15 min. the solution is cooled and poured into $200 \mathrm{ml}$ of $\mathrm{H}_{2} \mathrm{O}$. After standing overnight the mixture is filtered and the solids dissolved in $\mathrm{CHCl}_{3}$ and chromatographed on alumina eluting with $\mathrm{CHCl}_{3}$ giving $580 \mathrm{mg}\left(72 \%\right.$ yield) after treatment with $\mathrm{Na}_{2} \mathrm{SO}_{4}$ and drying. ${ }^{19} \mathrm{FNMR}$ $\left(\mathrm{CDCl}_{3}\right.$ referenced to $\left.\mathrm{C}_{6} \mathrm{D}_{5} \mathrm{~F}\right): \delta-114.4,-119.2$ (broad resonances, 8F, $\mathrm{Q}-\mathrm{ArF}$ ), $-148.4(4 \mathrm{~F}, \mathrm{p}-\mathrm{ArF}$ ), 157.1 (8F, m-ArF). LDMS:M-Cl ${ }^{+} 1304, \mathrm{M}^{+} 1339$. 
$\mathrm{Fe}\left(\mathrm{TPPF}_{20} \beta-\mathrm{Cl}_{8}\right) \mathrm{N}_{3}$. This azide complex was prepared by reacting $100 \mathrm{mg}$ of $\mathrm{Fe}\left(\mathrm{TPPF}_{20} \mathrm{\beta}-\mathrm{Cl}_{8}\right) \mathrm{Cl}$ with $1.0 \mathrm{~g}$ of $\mathrm{NaN}_{3}$ in $200 \mathrm{ml}$ of acetone with vigorous stirring for 24 hours. The product mixture was filtered. The filtrate was evaporated to dryness and the remaining solid washed with water. The solid product was then dried in vacue at $70^{\circ} \mathrm{C}$. The infrared spectrum shows a sharp $v_{\mathrm{N}-\mathrm{N}}$ stretch at $2037 \mathrm{~cm}^{-1}$.

$\operatorname{Cr}\left(\operatorname{TPPF}_{20} \beta-\mathrm{Cl}_{8}\right) \mathrm{Cl}$. A $250 \mathrm{ml}$ flask containing a solution of $\mathrm{H}_{2} \operatorname{TPPF}_{20} \beta-\mathrm{Cl}_{8}(750 \mathrm{mg}, 0.60$ $\mathrm{mmol}$ ) in $200 \mathrm{ml}$ DMF under a nitrogen atmosphere was placed in an oil bath preheated to $160^{\circ} \mathrm{C}$. After the solution had started refluxing, $\operatorname{Cr}(\mathrm{CO})_{6}(132 \mathrm{mg}, 0.60 \mathrm{mmol})$ was added every $30 \mathrm{~min}$. until the chromium insertion was judged to be complete by TLC. The reaction mixture was cooled to room temperature under a $\mathrm{N}_{2}$ atmosphere. At this point the solution was poured into $200 \mathrm{ml}$ of cold saturated $\mathrm{NaCl}$ solution and stirred for $10 \mathrm{~min}$. The brown flocculate was isolated from the aqueous solution by vacuum filtration. The resulting brown paste was dissolved in $20 \mathrm{ml} \mathrm{CHCl}_{3}$ and chromatographed on $\mathrm{Al}_{2} \mathrm{O}_{3}$ with $\mathrm{CHCl}_{3}$ steadily enriched with methanol. The product was the second porphyrin component which came off the column and eluted as a broad red-brown band and was treated in the normal fashion with $1 \mathrm{NHCl}$. The solvent was removed by rotary-evaporation to give $\mathrm{Cr}\left(\mathrm{TPPF}_{20} \beta-\mathrm{Cl}_{8}\right) \mathrm{Cl}$ in $30 \%$ yield. LDMS: $\mathrm{M}^{+}-\mathrm{Cl} 1299, \mathrm{M}^{+} 1336 . \mathrm{UV} / \mathrm{VIS}\left(\mathrm{CHCl}_{3}\right): 432 \mathrm{~nm}$.

$\operatorname{Cr}\left(\mathrm{TPPF}_{20} \beta-\mathrm{Cl}_{8} \mathrm{~N}_{3}\right.$. The complex, $\operatorname{Cr}\left(\mathrm{TPPF}_{20} \beta-\mathrm{Cl}_{8}\right) \mathrm{Cl}, 96 \mathrm{mg}$, and sodium azide, $1.0 \mathrm{~g}$. were stirred in $200 \mathrm{ml}$ of acetone at room temperature for 72 hours under nitrogen. After this time the solution was filtered. The filtrate was evaporated to dryness giving a solid product which was washed thoroughly with water and dried in vacuo. The purple azide complex $\left(v_{\mathrm{N}-\mathrm{N}} 2055 \mathrm{~cm}^{-1}\right)$ was recovered in quantitative yield. UV/VIS $\left(\mathrm{CHCl}_{3}\right): 418,440 \mathrm{~nm}$.

$\mathrm{Mn}\left(\mathrm{TPPF}_{20} \beta-\mathrm{Cl}_{8}\right) \mathrm{Cl}$. The free base, $\mathrm{H}_{2} \mathrm{TPPF}_{20} \beta-\mathrm{Cl}_{8}, 300 \mathrm{mg}$, was dissolved in $33 \mathrm{ml}$ of DMF. The solution was stirred at reflux under nitrogen for 15 minutes. At this point $\mathrm{Mn}(\mathrm{OAc})_{2} \bullet 4 \mathrm{H}_{2} \mathrm{O}, 200$ $\mathrm{mg}$. was added and the mixture was stirred at reflux under nitrogen for an additional 30 minutes. An additional $50 \mathrm{mg}$ of $\mathrm{Mn}(\mathrm{OAc})_{2}{ }^{\bullet} 4 \mathrm{H}_{2} \mathrm{O}$ was added and the mixture refluxed an additional 30 minutes. At this point an aliquot was taken, evaporated to dryness, and the solid taken up in $\mathrm{CHCl}_{3}$. A TLC check of this solution revealed no starting porphyrin. The DMF solution of manganese complex was then allowed to cool to room temperature and added to $50 \mathrm{ml}$ of a 30 weight \% solution of sodium chloride in water. The mixture was refrigerated overnight during which time the product precipitated from solution. The solid product was washed with water $(5 \times 20 \mathrm{ml})$, filtered, air dried for a hour then dissolved in a minimum of $\mathrm{CHCl}_{3}$. The $\mathrm{CHCl}_{3}$ solution was chromatographed on alumina then the product was taken to dryness in a rotary evaporator. The solid product was dried at $80^{\circ} \mathrm{C}$ in a vacuum oven. Recovered was $240 \mathrm{mg}$ of product having a Soret at $450 \mathrm{~nm}$.

$\mathrm{Mn}\left(\mathrm{TPPF}_{20} \beta-\mathrm{Cl}_{8}\right) \mathrm{N}_{3}$. To a round-bottom flask under $\mathrm{N}_{2}$ is charged $100 \mathrm{mg}$ of $\mathrm{Mn}\left(\mathrm{TPPF}_{20} \beta-\mathrm{Cl}_{8}\right) \mathrm{Cl}, 300 \mathrm{ml}$ of acetone, $50 \mathrm{ml}$ of methanol, and $1.0 \mathrm{~g}$ of $\mathrm{NaN}_{3}$. This mixture is stirred vigorously at room temperature for $12 \mathrm{hrs}$., filtered and the solvent removed by rotary evaporation. The solids are dissolved in $50 \mathrm{ml}$ of $\mathrm{CH}_{2} \mathrm{Cl}_{2}$ and washed twice with $25 \mathrm{ml}$ of $\mathrm{H}_{2} \mathrm{O}$ after which the organic layer is dried over $\mathrm{Na}_{2} \mathrm{SO}_{4}$. The solvent is removed by rotary evaporation and the purple 
solids dried in vacuo overnight at room temperature. The yield is quantitative. $\operatorname{IR}(\mathrm{KBr}): \mathrm{v}_{\mathrm{N}-\mathrm{N}}=2031$ $\mathrm{cm}^{-1}$ with a shoulder at $2066 \mathrm{~cm}^{-1}$.

$\mathrm{Co}\left(\mathrm{TPPF}_{20} \beta-\mathrm{Cl}_{8}\right)$. A stirred solution of $200 \mathrm{mg}$ of $\mathrm{Co}(\mathrm{OAc})_{2} \cdot 4 \mathrm{H}_{2} \mathrm{O}$ in $50 \mathrm{ml}$ of DMF is heated to $140^{\circ}$ in an oil bath then $250 \mathrm{mg}$ of the free base, $\mathrm{H}_{2} \mathrm{TPPF}_{20} \beta-\mathrm{Cl}_{8}$ is added as a powder all at once. After $15 \mathrm{~min}$. an aliquot is taken, evaporated to dryness and a TLC check is made on alumina eluting with $\mathrm{CHCl}_{3}$. No free base is seen by TLC. The total reaction time is limited to $30 \mathrm{~min}$. The contents of the flask are cooled quickly to room temperature and added to an equal volume of saturated $\mathrm{NaCl}$ solution. The resulting precipitate was filtered giving a reddish-brown paste which is redissolved in $\mathrm{CHCl}_{3}$ and chromatographed on neutral alumina eluting with $\mathrm{CHCl}_{3}$. After evaporation of the solvent and drying in vacuo at $110^{\circ}, 210 \mathrm{mg}$ of the product is obtained. UV/VIS $\left(\mathrm{CHCl}_{3}\right): 427,552,578 \mathrm{~nm}$. LDMS:M+ 1307 .

$\mathrm{Co}\left(\mathrm{BPI} \mathrm{N}_{3}\right.$. In an erlenmeyer flask under $\mathrm{N}_{2}$ is stirred $0.5 \mathrm{~g}$ of $\mathrm{Co}(\mathrm{BPI})(\mathrm{OAc})$ in $200 \mathrm{ml}$ of methanol. At reflux $0.6 \mathrm{~g}$ of $\mathrm{NaN}_{3}$ is added and reflux is continued for $1 \mathrm{hr}$. After cooling and filtering, the volume is reduced to $100 \mathrm{ml}$ then an equal volume of ether is added to precipitate the product which is washed with water, then ether and then dried in vacuo overnight at room temperature. Yield is quantitative. $\operatorname{IR}(\mathrm{KBr}): v_{\mathrm{N}-\mathrm{N}}=2015 \mathrm{~cm}^{-1}$.

$\mathrm{Fe}\left(\mathrm{TPP} \beta-\mathrm{Br}_{4}\right) \mathrm{N}_{3}$. In a round-bottomed flask under $\mathrm{N}_{2}$ is charged $100 \mathrm{mg}$ of $\mathrm{Fe}\left(\mathrm{TPP} \beta-\mathrm{Br}_{4}\right) \mathrm{Cl}$ and $100 \mathrm{ml}$ of $\mathrm{CHCl}_{3}$. With stirring $1.0 \mathrm{~g}$ of $\mathrm{NaN}_{3}$ in $50 \mathrm{ml}$ of $\mathrm{H}_{2} \mathrm{O}$ and 5 drops of $\mathrm{H}_{2} \mathrm{SO}_{4}$ is added. After stirring for $12 \mathrm{hr}$. the $\mathrm{CHCl}_{3}$ is separated, filtered and washed twice with water. The $\mathrm{CHCl}_{3}$ layer is reduced in volume by rotary evaporation until a precipitate forms. The material is filtered and washed with $\mathrm{H}_{2} \mathrm{O}$ then dried in vacuo. Yield is $90 \mathrm{mg}$. UV/Vis $\left(\mathrm{CHCl}_{3}\right): 433,523,602 . \mathrm{R}(\mathrm{KBr}): \mathrm{V}_{\mathrm{N}-\mathrm{N}}$ $=2030 \mathrm{~cm}^{-1}$.

$\mathrm{Fe}\left(\mathrm{TPPCl}_{8}\right) \mathrm{N}_{3}$. In an erlenmeyer flask under $\mathrm{N}_{2}$ is dissolved $200 \mathrm{mg}$ of $\mathrm{Fe}\left(\mathrm{TPPCl}_{8}\right) \mathrm{Cl}$ in $100 \mathrm{ml}$ of $\mathrm{CHCl}_{3}$. To this is added $2 \mathrm{~g}$ of $\mathrm{NaN}_{3}$ dissolved in $40 \mathrm{ml}$ of $\mathrm{H}_{2} \mathrm{O}$ and 5 drops of $\mathrm{H}_{2} \mathrm{SO}_{4}$. This two phase mixture is stirred vigorously for $24 \mathrm{hr}$. then the $\mathrm{CHCl}_{3}$ layer is separated, dried over $\mathrm{Na}_{2} \mathrm{SO}_{4}$ and evaporated to dryness. The purple solids are washed with water and dried in vacuo. The yield is 150 mg. $\mathbb{I R}(\mathrm{KBr}): v_{\mathrm{N}-\mathrm{N}}=2055 \mathrm{~cm}^{-1}$.

$\mathrm{Fe}\left(\mathrm{TPPCl}_{8} \beta-\mathrm{Br}_{4}\right) \mathrm{N}_{3}$. A quantity of $100 \mathrm{mg}$ of $\mathrm{Fe}\left(\mathrm{TPPCl}_{8} \beta-\mathrm{Br}_{4}\right) \mathrm{Cl}$ is dissolved in $100 \mathrm{ml}$ of $\mathrm{CHCl}_{3}$ and 1.0 of $\mathrm{NaN}_{3}$ dissolved in $20 \mathrm{ml}$ of $\mathrm{H}_{2} \mathrm{O}$ with a few drops of $\mathrm{H}_{2} \mathrm{SO}_{4}$ is added. This two phase mixture is stirred vigorously for $24 \mathrm{hrs}$, then the organic layer is separated, washed twice with $\mathrm{H}_{2} \mathrm{O}$ and dried over $\mathrm{Na}_{2} \mathrm{SO}_{4}$. After removal of the solvent the solids were washed with $\mathrm{H}_{2} \mathrm{O}$ and dried in vacue at room temperature. The yield is $80 \mathrm{mg}$ of purple powder. $\operatorname{IR}(\mathrm{KBr}): v_{\mathrm{N}-\mathrm{N}}=2050 \mathrm{~cm}^{-1}$. 


\subsection{ABBREVIATIONS}

TPP

$\mathrm{TPP} \beta-\mathrm{Br}_{4}$

$\mathrm{TPPCl}_{8}$

$\mathrm{TPPCl}_{8} \beta-\mathrm{Br}_{4}$

$\mathrm{TPPF}_{20}$

$\mathrm{TPPF}_{20} \beta-\mathrm{Cl}_{8}$

$\mathrm{TPPF}_{20} \beta-\mathrm{Br}_{8}$ meso-tetraphenylporphyrin dianion

meso-tetraphenyl $\beta$-tetrabromoporphyrin dianion

meso-tetrakis(2,6-dichlorophenyl)porphyrin dianion

mesa-tetrakis(2,6-dichloropheny)B-tetrabromo porphyrin dianion mesa-tetrakis(pentafluorophenyl)porphyrin dianion meso-tetrakis(pentafluorophenyl)- $\beta$-octachloroporphyrin dianion meso-tetrakis(pentafluorophenyl)- $\beta$-octabromoporphyrin dianion

\subsection{REFERENCES}

1.a. Lyons, J. E., "Surface Organometallic Chemistry:Molecular Approaches to Surface Catalysis", J. M. Basset, ed., Kluwer Academic Publishers, 97 (1988).

b. Lyons, J. E., "Applied Industrial Catalysis", B. E. Leach, ed., Ch. 6, Vol. 3, Academic Press, New York, 131 (1984).

c. Lyons, J. E., FHdrocarbon Processing, 107 (1980).

2. Groves, J. T., Nemo, T. E., and Myers, R. S., 9. Am. Chem. Soc, 98,859 (1976).

3. Groves, J. T., Nemo, T. E., and Myers, R. S., J. Am. Chem. Soc, 101,1032 (1979).

4. Groves, J. T. and Kruper, W. J., Jr., 9. Am. Chem. Soc, 101, 7613 (1979).

5. Meunier, B., Chem. Rev, 92, 1411 (1992).

6. Mansuy, D., Bartoli, J. F., Chottard, J. C. and Lange, M., Agnew. Chem Int. Ed. Eng., 19, 909 (1980).

7. Hill, C. L. and Schardt, B.C., J. Am. Chem. Soc, 102, 6374 (1980).

8. Chang, C. K. and Ebina, F., I. Cherm. Soc. Chem Comm, 778 (1981).

9. Groves, T. and Nemo, T. E., I. Am. Chem. Soc, 105, 6243 (1983).

10. DePorter, B., Ricci, M., Bortolino, O., and Meunier, B., J. Mol. Cat., 31, 221 (1985).

11. Tabushi, I. and Yazaki, A., I. Am Chem Soc, 103, 7371 (1981). 


\subsection{REFERENCES}

(CONTINUED)

12. Tabushi, I. and Koga, N., 9. Am. Chem. Soc, 101, 6456 (1979).

13. Tabushi, I., Coord. Chem Rev, 86, 1 (1988).

14. Mansuy, D., Fontecave, M., and Bartoli, J. F., g. Chem. Soc Chem. Comm, 253 (1983).

15. Fontecave, M. and Mansuy, D., Tetrahedron, 40, 4297 (1984).

16. Ji, L., Liu, M., Hsieh, A. K., and Hor, T. S. A., J. Mol Cat., 70, 247 (1991).

17. Iwanejko, R., Mlodnicka, T., and Poltowicz, J., "New Developments in Selective Oxidation," (G. Centi and F. Trifiro, eds.), Elsevier, Amsterdam, 195 (1990).

18. Battioni, P., Bartoli, J. F., Leduc, P., Fontecave, M., and Mansuy, D., J. Chem. Soc. Chem Comm, 791 (1987).

19. Karasevich, E. I., Khenkin, A. M.,and Shilov, A.E., g. Chem. Soc. Chem. Comm., 731 (1987).

20. Shulpin., G. B. and Druzhinina, A. N., Izv. AKad. NauK SSSR Ser. Khim, 2739 (1991).

21. Leduc, P., Battioni, P., Bartoli, J.F. and Mansuy, D., Tett. Lett., 29, 205 (1988)

22. Bedioui, F., Granados, S. G., and Devynck, J., New g. Chem, 15, 939 (1991).

23. Hendrickson, D. N., Kinnaird, M. G., and Suslick, K. S., I. Am. Chem. Soc, 109, 1243 (1987).

24. Maldotti, A., Bartocci, C., Amadelli, R., Polo, E., Battioni, P., and Mansuy, D., g. Chem. Soc. Chem. Comm., 1487 (1991).

25. Ellis, P. E., Jr. and Lyons, J. E., J. Chem. Soc. Chem Commum, 1188 (1989).

26. Ellis, P. E., Jr. and Lyons, J. E., J. Chem. Soc, Chem. Commun, 1190 (1989).

27. Ellis, P. E., Jr. and Lyons, J. E., J. Chem. Soc., Chem. Commun, 1316 (1989).

28. Ellis, P. E., Jr. and Lyons, J. E., Catal. Lett, 3, 389 (1989). 


\subsection{REFERENCES (CONTINUED)}

29. Lyons, J. E., and Ellis, P. E., Jr., Catal. Lett., 8, 45 (1991).

30. Ellis, P. E., Jr. and Lyons, J. E., Coord. Cherm. Rev., 105, 181 (1990).

31. Lyons, J. E., Ellis P. E., Jr., and Durante, V.A., "Studies in Surface Science and Catalysis," 67, R. Grasselli, ed., Elsevier, New York, 99 (1991).

32. Ellis, P. E., Jr. and Lyons, J. E., Preprints Petr. Div., 35, 174 (1990).

33. Lyons, J. E., Ellis, P. E., Jr., Wagner, R. W., Thompson, P. B., Gray, H. B., Hughes, M. E., and Hodge, J. A., Preprints Petr. Div, 37, 307 (1992).

34. Lyons, J. E., and Ellis, P. E., Jr., "Metalloporphyrins in Catalytic Oxidations," R. A. Sheldon, ed., Marcel Dekker, New York, 291-324 (1994).

35. Bartoli, J. F., Brigoud, O., Battioni P., and Mansuy, D., 9. Chem Soc, Chem Commum, 440 (1991)

36. Bartoli, J. F., Battioni, P., DeFoor, W. R., and Mansuy, D., J. Chem. Soc. Chem. Commun., 23 (1994).

37. Mansuy, D., "The Activation of Dioxygen and Homogeneous Catalytic Oxidation," D. H R. Barton et. al., ed., Plenum Press, New York, 347-358 (1993).

38. Grinstaff, M. W., Hill, M. G., Labinger, J., and Gray, H.B., Science, 264, 1311 (1994).

39. Tolman, C. A., Druliner, J. P., Krusic, P. J., Nappa, M. J., Seidel, W.C., Williams, I. D., and Ittel, S.D., J. MoL Catah, 48, 129 (1988).

40. Saussine, L., Brazi, E., Robine, A., Mimoun, H., Fischer J., and Weiss, R., I. Am Chem. Soc, 107, 3534 (1985).

41. Groves, J. T., Adv. Inorg. Biochem, 1, 119 (1979).

42. White, R. E., and Coon, M. J., Ann Rev. Biochem, 49, 315 (1980).

43. Guengerich F. P., and MacDonald, T., Acc. Chem. Res, 17, 9 (1984).

44. Hamilton, G. A., in "Molecular Mechanisms of Oxygen Activation", O. Hayaishi, ed, Academic Press New York 405 (1974). 


\subsection{REFERENCES}

(CONTINUED)

45. Ortiz de Montellano P. R., in "Cytochrome P-450," P. R. Ortiz de Montellano, ed.), Plenum Press,

New York, 235-239 (1986).

46. McKenna E. J., and Coon, M. J., J. Biol. Chem, 245, 3882 (1970).

47. Colby J. and Dalton, H., Biochem. J., 157, 495 (1976).

48. Woodland, M. P., and Dalton, H. J., J. BioL Chem, 259, 53 (1984).

49. Balch, A. L., Chen, Y. W., Cheng, R. J., LaMar, G. N., Latos-Grazynski, L., and Renner, M. W., g. Am. Chem Soc, 106, 7779 (1984).

50. Reviewed in Momenteau, M. and Reed C. A., Chem. Rev., 94, 659 (1994).

51. Ozawa, S., Watanabe, Y., Nakashima, S., Kitigawa T., and Morishima, I., J. Am. Chem. Soc, 116,634 (1994).

52. Paulson, D.R., Ulliman, R., and Sloane, R. B, J. Chem. Soc. Chem. Comm,186 (1974).

53. Dolphin, D. and Felton, R. H., Acc. Chem. Res., 7, 26 (1973).

54. Traylor, P. S., Dolphin, D., and Traylor, T. G., J. Chem. Soc. Chem. Comm, 279 (1984).

55. Chin, D., Balch, A.L., and LaMar, G. N., 9. Am. Chem. Soc, 102, 1446 (1980).

56. Al-Malaika, D., "Atmospheric Oxidation and Antioxidants", G. Scott, ed., Elsevier, New York, 45-82 (1993).

57. Howard, J., "Free Radicals vol. II", J. Kochi,ed., Wiley, New York, 3 (1970).

58. Howard, J., Chenier J., and Holden, D., Can I. Chem., 56, 170 (1978).

59. Lyons, J. E., Ellis, P. E., Jr., Myers, H. K., and Wagner, R. W., I. Catah, 141, 311 (1993).

60. Labinger, J. A., Catal, Leth, 26, 95 (1994).

61. Kochi, J. K., "Free Radicals, Vol. II," Wiley, New York, 683-686 (1973). 


\subsection{REFERENCES}

(CONTINUED)

62. Greene, F. D., Savitz, M.L., Osterholtz, F. D., Lau, H. H., Smith W. N., and Zanet, P. M.,J. Otg. Chem. 28, 55 (1963).

63. Summerville, D.A., Jones, R.D., Hoffman, B.M. and Basolo, F., J. Am. Chem. Soc, 99, 8195 (1977).

64. Buchler, J. W. and Dreher, C., Z. Naturforsch, 396, 222 (1984).

65. Summerville, D.A. and Cohen, I. A., J. Am. Chem. Soc, 98, 1747 (1976).

66. Ledon, H., C. R. Acad. Sci.(Paris), 288, C29 (1979).

67. Donohoe, R.J., Atamian, M., and Bocian, D.F., J.Am. Chem. Soc, 109, 5593 (1987).

68. Traylor, P.S., Dolphin, D., and Traylor, T. G., I. Chem. Soc. Chem. Comm, 279 (1984).

69. Jayaraj, K., Gold, A., Toney, G. E., Helms, J. H. and Hatfield, W. E., Inorg. Chem, 25, $3516(1986)$.

70. Liston, D. J. and West, B.O., Inotg. Chem, 24, 1568 (1985).

71. Banfi, S., Montanari, F., and Quici, S., g. Otg. Chem, 53, 2863 (1988).

72. Siegl, W. O., J. Org. Chem., 42, 1872 (1977). 
Table 1 Effect of Fluorination of The meso-Phenyl Groups on The Activity of Tetraphenyporphyrinato Metal Complezes for Reactions of Isobutane with Oxygen

\begin{tabular}{|c|c|c|c|}
\hline Catalyst & $\begin{array}{l}\text { mmoles } \\
\text { Catalyst }\end{array}$ & $\begin{array}{l}\text { Catalyst } \\
\text { Tumovers }^{b}\end{array}$ & $\begin{array}{c}\text { Selectivity to } \\
\text { tert-Butyl Alcohol. } \%\end{array}$ \\
\hline $\mathrm{Fe}(\mathrm{TPP}) \mathrm{Cl}$ & 0.025 & 0 & - \\
\hline $\mathrm{Fe}\left(\mathrm{TPPF}_{2 \mathrm{D}}\right) \mathrm{Cl}$ & 0.016 & 2040 & 90 \\
\hline $\mathrm{Fe}(\mathrm{TPP}) \mathrm{N}_{3}$ & 0.013 & 130 & 93 \\
\hline $\mathrm{Fe}\left(\mathrm{TPPF}_{z 0}\right) \mathrm{N}_{3}$ & 0.016 & 2060 & 89 \\
\hline $\mathrm{Mn}(\mathrm{TPP}) \mathrm{N}$ & 0.013 & 180 & .88 \\
\hline $\mathrm{Mn}\left(\mathrm{TPPF}_{90}\right) \mathrm{N}$ & 0.016 & 750 & 87 \\
\hline $\mathrm{Cr}(\mathrm{TPP}) \mathrm{N}_{3}$ & 0.025 & 280 & 89 \\
\hline $\operatorname{Cr}\left(T_{P P F_{: 0}}\right) \mathrm{N}_{3}$ & 0.016 & 450 & 97 \\
\hline$[\mathrm{Fe}(\mathrm{TPP})]_{:} \mathrm{O}$ & 0.013 & 0 & - \\
\hline$\left[\mathrm{Fe}\left(\mathrm{TPPF}_{: \dot{ }}\right)\right]_{:} \mathrm{O}$ & 0.007 & 1730 & 92 \\
\hline
\end{tabular}

${ }^{4}$ Isobutane. $7 \mathrm{~g}$, was oxidized ( $6 \mathrm{hrs}$ ) in $25 \mathrm{ml}$ benzene containing the designated catalyst at $80^{\circ} \mathrm{C}$ under 100 psig oxygen. Moles of oxygen consumed/mole catalyst used. [moles tert-butyl alcohoumoles liquid product] $\times 100$. 
Table 2 Effect of Ring Halogenation on The Isobutane Oridation Activity of Porphyrinatoiron(III) Complexes"

\begin{tabular}{|c|c|c|c|c|}
\hline \multirow[b]{2}{*}{ Catalyst } & \multicolumn{3}{|c|}{ O. Uprake } & \multirow{2}{*}{$\begin{array}{l}\text { Selectivity } \\
\text { to IBA \% }\end{array}$} \\
\hline & mmoles & mmoles & I. & \\
\hline $\mathrm{Fe}(\mathrm{TPP}) \mathrm{Cl}$ & 0.025 & 0.0 & 0 & - \\
\hline $\mathrm{Fe}\left(\mathrm{TPP} \beta-\mathrm{Br} r_{s}\right) \mathrm{Cl}$ & 0.013 & 2.0 & 155 & - \\
\hline $\mathrm{Fe}\left(\mathrm{TPPCl}_{\mathrm{g}}\right) \mathrm{Cl}$ & 0.019 & 5.0 & 263 & 89 \\
\hline $\mathrm{Fe}\left(\mathrm{TPPCl} \mathrm{g}_{8} \beta-\mathrm{Br}_{4}\right) \mathrm{Cl}$ & 0.020 & 17.3 & 865 & 83 \\
\hline $\mathrm{Fe}\left(\mathrm{TPPF}_{20}\right) \mathrm{Cl}$ & 0.016 & 32.6 & 2,040 & 90 \\
\hline $\mathrm{Fe}\left(\mathrm{TPPF}_{30} \beta-\mathrm{Br}_{8}\right) \mathrm{Cl}$ & 0.013 & 40.2 & 3,090 & 89 \\
\hline $\mathrm{Fe}(\mathrm{TPP}) \mathrm{N}_{3}$ & 0.013 & 1.7 & 130 & 89 \\
\hline $\mathrm{Fe}\left(\mathrm{TPP} \beta-\mathrm{Br}_{4}\right) \mathrm{N}_{3}$ & 0.013 & 2.3 & 177 & - \\
\hline $\mathrm{Fe}\left(\mathrm{TPPCl}_{3}\right) \mathrm{N}_{3}$ & 0.023 & 15.0 & 653 & 80 \\
\hline $\mathrm{Fe}\left(\mathrm{TPPCl}, \beta-\mathrm{Br}_{2}\right) \mathrm{N}_{3}$ & 0.023 & 21.5 & 934 & 82 \\
\hline $\mathrm{Fe}\left(\mathrm{TPPF}_{20}\right) \mathrm{N}_{3}$ & 0.016 & 33.0 & 2,060 & 89 \\
\hline$[\mathrm{Fe}(\mathrm{TPP})]=\mathrm{O}$ & 0.019 & 0.0 & 0 & - \\
\hline$\left[\mathrm{Fe}\left(\mathrm{TPP} \beta-\mathrm{Br}_{4}\right)\right]: \mathrm{O}$ & 0.013 & 0.0 & 0 & - \\
\hline $\mathrm{Fe}(\mathrm{TPPCl},) \mathrm{OH}$ & 0.013 & 9.2 & 711 & 83 \\
\hline$\left[\mathrm{Fe}\left(\operatorname{TPPF}_{z 0}\right)\right]_{2} \mathrm{O}$ & 0.013 & 24.0 & 1,846 & 84 \\
\hline $\mathrm{Fe}\left(\mathrm{TPPF}_{: 0}\right) \mathrm{OH}$ & 0.013 & 29.2 & 2,245 & 82 \\
\hline
\end{tabular}




\begin{tabular}{|c|c|c|c|}
\hline Catalyst & $\mathrm{I}^{\circ} \mathrm{C}$ & I. ${ }^{b}$ & $\begin{array}{r}\text { t-BuOH } \\
\text { Sel.\% }\end{array}$ \\
\hline \multirow[t]{3}{*}{$\mathrm{Fe}\left(\mathrm{TPPF}_{20} \mathrm{~B}-\mathrm{Br}_{8}\right) \mathrm{N}_{3}$} & 60 & 1550 & 87 \\
\hline & 40 & 670 & 89 \\
\hline & 27 & 620 & na \\
\hline \multirow[t]{2}{*}{$\mathrm{Fe}\left(\mathrm{TPPF}_{30} \mathrm{\beta}-\mathrm{Cl}_{8}\right) \mathrm{N}_{3}$} & 60 & 1800 & 90 \\
\hline & 40 & 760 & 92 \\
\hline $\operatorname{Cr}\left(\mathrm{TPPF}_{20} \beta-\mathrm{Br}_{8}\right) \mathrm{N}_{3}$ & 60 & 110 & 92 \\
\hline $\mathrm{Cr}\left(\mathrm{TPPF}_{20} \mathrm{~B}-\mathrm{Cl}_{8}\right) \mathrm{N}_{3}$ & 80 & 450 & 88 \\
\hline $\mathrm{Mn}\left(\mathrm{TPPF}_{20} \beta-\mathrm{Br}_{8}\right) \mathrm{N}_{3}$ & 60 & 270 & 87 \\
\hline $\mathrm{Mn}\left(\mathrm{TPPF}_{20} \beta-\mathrm{Cl}_{8}\right) \mathrm{Cl}+\mathrm{NaN}_{3}^{\mathrm{c}}$ & 80 & 1289 & 86 \\
\hline $\mathrm{Mn}\left(\mathrm{TPPF}_{20} \beta-\mathrm{Cl}_{8}\right) \mathrm{Cl}+\mathrm{NaN}_{3}^{\circ}$ & 60 & 156 & 88 \\
\hline $\mathrm{Co}\left(\mathrm{TPPF}_{20} \beta-\mathrm{Cl}_{8}\right)$ & 60 & 355 & 91 \\
\hline
\end{tabular}

Isobutane $(7 \mathrm{~g})$ was oxidized ( $6 \mathrm{hrs}$ ) in $25 \mathrm{ml}$ benzene at the designated temperature under 100 psig oxygen using 0.013 mmole of the designated catalyst. ${ }^{b}$ Moles of oxygen consumed/mole catalyst used. ${ }^{c}$ After warming the reaction mixture for $6 \mathrm{hr}$. as indicated in footnote a, the reaction mixture was cooled to room temperature, $10 \mathrm{mg}$ of $\mathrm{NaN}_{3}$ added and the procedure repeated according to footnote a. The T.O. are calculated based on the second $6 \mathrm{hr}$. period. 
Table 4 Propane Oxidations Using First Row Metal Complezes of TPPF 20 B-Br, as Catalysts"

\begin{tabular}{|c|c|c|c|c|}
\hline Carajyst. & time hours & $I^{\circ} \mathrm{C}$ & I..$^{\circ}$ & IPA/Acetone \\
\hline Fe(TPPF $: 2) N_{2}$ & 3 & 125 & 330 & 0.8 \\
\hline $\mathrm{Fe}\left(\mathrm{TPPF}{ }_{z \rho} \beta-\mathrm{Br}_{8}\right) \mathrm{N}_{2}$ & 45 & 125 & 541 & 0.9 \\
\hline $\operatorname{Cr}\left(\mathrm{TPPF}_{: 2}\right) \mathrm{N}_{i}$ & 45 & 125 & 41 & $<0.1$ \\
\hline CriTPPF $\left.{ }_{2} \beta-\mathrm{Br}_{8}\right) \mathrm{N}_{3}$ & 45 & 125 & 87 & 0.6 \\
\hline. $\mathrm{Ln}\left(\mathrm{TPPF}_{: \mathrm{o}}\right) \mathrm{Ni}_{2}$ & 3 & 125 & 0 & - \\
\hline MniTPPF $\left.: \beta-B r_{8}\right) N_{i}$ & 45 & 125 & 87 & 1.0 \\
\hline
\end{tabular}

${ }^{4}$ Propane ( $\left.1.36 \mathrm{~mol}\right)$ was added to benzene $(48 \mathrm{ml})$ containing the catalyst $(0.013 \mathrm{mmol})$. The soiution was stirred for 3 hours at $125^{\circ} \mathrm{C}$ under 1000 psig of air in a glass lined autoclave . 'Moies of acetone plus isopropyl alcohol formed per mole of catalyst used. 'Molar ratio isopropyl alcohol to acetone formed 
Table 5 Alkane ()xidations (ialalyzed by Cobalt Complexes

\begin{tabular}{|c|c|c|c|c|c|c|c|c|c|}
\hline \multirow[b]{2}{*}{ Complex } & \multirow{2}{*}{$\begin{array}{l}\text { mmoles } \\
\text { Complex }\end{array}$} & \multirow[b]{2}{*}{ Substrale } & \multirow{2}{*}{$\begin{array}{c}\text { Reaction } \\
\text { T."C }\end{array}$} & \multirow{2}{*}{$\begin{array}{l}\text { Total } \\
\text { Posis. }\end{array}$} & \multirow{2}{*}{$\begin{array}{c}\text { Reaction } \\
\text { 1, hrs }\end{array}$} & \multirow{2}{*}{$\begin{array}{l}\text { Catalyst } \\
\text { Turnovers }\end{array}$} & \multicolumn{3}{|c|}{ Product, Selectivity, $\%$} \\
\hline & & & & & & & $\underline{T B A-H P}^{c}$ & Acetone & $\mathbb{P A}$ \\
\hline $\mathrm{Co}(\mathrm{acac})_{2}$ & 0.100 & $i-C_{4} H_{10}$ & 80 & 100 & 6 & 52 & 89 & 10 & - \\
\hline $\mathrm{Co}(\mathrm{acac})_{3}$ & 0.100 & & & & & 15 & 88 & 10 & - \\
\hline $\mathrm{Co}(\mathrm{BPI})(\mathrm{OAc})$ & 0.025 & & & & & 24 & 88 & 12 & - \\
\hline $\mathrm{Co}(\mathrm{BPI})\left(\mathrm{O}_{2} \mathrm{Bu}^{\prime}\right)$ & 0.025 & & & & & 260 & 89 & 11 & - \\
\hline $\mathrm{Co}(\mathrm{acac})_{2}$ & 0.023 & $\mathrm{C}_{3} \mathrm{H}_{8}{ }^{h}$ & 125 & 1,000 & 3 & 21 & - & 62 & 35 \\
\hline $\mathrm{Co}(\mathrm{acac})_{2}$ & 0.023 & & & & 18 & 122 & - & 62 & 35 \\
\hline $\mathrm{Co}(\mathrm{BPI})\left(\mathrm{O}_{2} \mathrm{Bu}^{\prime}\right)$ & 0.023 & & & & 3 & 20 & - & $>90$ & $<10$ \\
\hline
\end{tabular}

a Isobutane, 7g, was oxidized as in Table 1. 'Propane, 60g, was oxidized as in Table 4. "mole percent (TBA + TBHP). 
Table 6 Iron IIaloporphyrin Catalyzed Isobutane Oxidation ${ }^{a}$

\begin{tabular}{|c|c|c|c|c|c|c|c|c|c|c|c|}
\hline \multirow[b]{2}{*}{ Catalys! } & \multirow[b]{2}{*}{$T^{\circ} \mathrm{C}$} & \multirow[b]{2}{*}{ t.Hrs. } & \multirow[b]{2}{*}{$\mathrm{i}-\mathrm{C}_{4} \mathrm{H}_{10}$ (mmols) } & \multirow[b]{2}{*}{$\underline{O}_{2}(\mathrm{psi})$} & \multicolumn{4}{|c|}{ Reaction Producis. (mmoles) } & \multirow{2}{*}{$\begin{array}{l}\text { Conversion } \\
-\mathrm{C}_{4} \mathrm{H}_{10}(\%)\end{array}$} & \multirow{2}{*}{$\begin{array}{c}\text { Sel \% } \\
\text { I-BuOH }^{b}\end{array}$} & \multirow[b]{2}{*}{$T . Q^{c}$} \\
\hline & & & & & I-BuOHI & Acetone & $\mathrm{CO}$ & $\mathrm{CO}_{2}$ & & & \\
\hline \multirow[t]{2}{*}{$\mathrm{Fe}\left(\mathrm{TPPF}_{20}\right) \mathrm{OH}$} & 80 & 3 & 1869 & 53 & 310 & 46 & 7 & 18 & 17 & $87^{\prime}$ & 11,330 \\
\hline & 24 & 143 & 1871 & 53 & 382 & 17 & 0 & 18 & 19 & 95 & 11,600 \\
\hline \multirow[t]{2}{*}{$\mathrm{Fe}\left(\mathrm{TPPF}_{20} \beta-\mathrm{Cl} \mathrm{l}_{8}\right) \mathrm{Cl}$} & 80 & 3 & 1865 & 142 & 334 & 38 & 6 & 29 & 20 & 88 & 12,400 \\
\hline & 21 & 120 & 1865 & 63 & 366 & 16 & tr & na & 20.5 & 95 & 12,730 \\
\hline \multirow[t]{3}{*}{$\mathrm{Fe}\left(\mathrm{TPPF}_{20} \beta-\mathrm{Br} r_{8}\right) \mathrm{Cl}$} & 80 & 3 & 1862 & 148 & 414 & 81 & 6 & 28 & 27 & 84 & 16,500 \\
\hline & 80 & 3 & 1870 & 53 & 277 & 43 & 8 & 23 & 17 & 87 & 10,060 \\
\hline & 24 & 71 & 1862 & 53 & 372 & 35 & tr & 27 & 22 & 92 & 12,150 \\
\hline
\end{tabular}

'Isobutane containing 0.03 mmole of dissolved catalyst was oxidized by an oxygen-containing gas mixture $\left(75\right.$ atm. diluent $\left.-\mathrm{N}_{2}\right)$ in the liquid phase $(180 \mathrm{ml})$ for 3 hours. Oxygen added as consumed ${ }^{\circ}$ (moles $\mathrm{t}-\mathrm{BuOH} / \mathrm{moles}$ liquid product) $\times 100$. ${ }^{\mathrm{M}} \mathrm{Moles}(\mathrm{t}-\mathrm{BuOH}$ and acetone) produced/mole catalyst used. 
Table 7 Conversion of tert-Butyl Hydroperoxide to tert-Butyl Alcohol

\begin{tabular}{|c|c|c|c|c|c|}
\hline \multirow[b]{2}{*}{ Catalyst } & \multirow{2}{*}{$\begin{array}{l}\text { time } \\
\text { hrs }\end{array}$} & \multirow{2}{*}{$\begin{array}{l}\mathrm{t}-\mathrm{BuO} \mathrm{H} \\
\text { Conv \% }\end{array}$} & \multicolumn{3}{|c|}{ Product. Molar Sel.\% } \\
\hline & & & $\mathrm{t}-\mathrm{BuOH}$ & $\left(t-\mathrm{BuO}_{2}\right.$ & $\left(\mathrm{CH}_{3}\right)_{2} \mathrm{CO}$ \\
\hline $\mathrm{Fe}(\mathrm{acac})_{3}$ & 2.3 & $<5$ & 67 & tr & 32 \\
\hline $\mathrm{Fe}(\mathrm{TPP}) \mathrm{Cl}$ & 1.9 & 27 & 82 & 7 & 11 \\
\hline $\mathrm{Fe}\left(\mathrm{TPPF}_{20}\right) \mathrm{Cl}$ & 3.3 & 72 & 87 & 10 & 3 \\
\hline $\mathrm{Fe}\left(\mathrm{TPPF}_{20} \beta-\mathrm{Br}_{8}\right) \mathrm{Cl}$ & 1.9 & 95 & 90 & 8 & 2 \\
\hline $\mathrm{Fe}\left(\mathrm{TPPF}_{20} \mathrm{\beta}-\mathrm{Cl}_{3}\right) \mathrm{Cl}$ & 3.3 & 100 & 90 & 8 & 2 \\
\hline
\end{tabular}

'The catalyst, $2 \times 10^{-4}$ mmoles, in $2.4 \mathrm{ml}$ p-xylene was rapidly added to a stirred solution of $10 \mathrm{ml}$ t- $\mathrm{BuO}_{2} \mathrm{H}(90 \%)$ in $48 \mathrm{mls}$ benzene. $\mathrm{O}_{2}$ evolved was measured manometrically and liquid samples taken periodically and analyzed by standardized $\mathrm{gc}$. 
Table 8 Relationship Between Catalyst Reduction Potential and Hydroperoxide Decomposition Activity

\begin{tabular}{|c|c|c|}
\hline Cataiysi & $\begin{array}{c}\mathrm{Fe}(\mathrm{III}) /(\mathrm{II})^{2} \\
\mathrm{E}_{1 / 2}(\mathrm{~V})\end{array}$ & $\begin{array}{l}\text { TBA yield } \\
\% \text { at } 0.5 \mathrm{Hr}\end{array}$ \\
\hline $\mathrm{Fe}(\mathrm{TPP}) \mathrm{Cl}$ & -0.221 & 4.8 \\
\hline $\mathrm{Fe}\left(\mathrm{TPPF}_{30}\right) \mathrm{Cl}$ & -0.07 & 16.0 \\
\hline $\mathrm{Fe}\left(\mathrm{TPPF}_{20} \beta-\mathrm{Br}_{3}\right) \mathrm{Cl}$ & +0.19 & 51.2 \\
\hline $\mathrm{Fe}\left(\mathrm{TPPF}_{20} \mathrm{\beta}-\mathrm{Cl}_{8}\right) \mathrm{Cl}$ & +0.28 & 67.2 \\
\hline
\end{tabular}

'Cyclic voltammetry in $\mathrm{CH}_{2} \mathrm{Cl}_{2}$ vs. SCE, TBAC-supporting electrolyte, glassy carbon electrode. "Room temperature decomposition of TBHP in Benzene. 
Table 9 n-Butane Oxidations Catalyzed By TPPF 20 - $\mathrm{Cl}_{8}$ Complexes of Iron and Chromium"

\begin{tabular}{|c|c|c|c|c|c|c|c|}
\hline \multirow{3}{*}{ Catalyst } & \multirow{3}{*}{ Solvent } & \multirow[b]{2}{*}{ React. } & \multirow{2}{*}{ TO } & \multicolumn{4}{|c|}{ Oxidation Product Molar Selectivity. \% } \\
\hline & & & & $\begin{array}{l}\text { SBA } \\
\left(2-\mathrm{C}_{4} \mathrm{H}_{0} \mathrm{OH}\right)\end{array}$ & $\begin{array}{c}\text { MEK } \\
(2-\mathrm{C}, \mathrm{H}, \mathrm{O})\end{array}$ & $\begin{array}{l}\text { Acetal- } \\
\text { dehyde } \\
\text { (CH, } \mathrm{CHO})\end{array}$ & $\begin{array}{c}\text { Acetic } \\
\text { acid } \\
\left(\mathrm{CH}_{2} \mathrm{CO}, \mathrm{H}\right)\end{array}$ \\
\hline & & & & & & & 1 \\
\hline $\mathrm{Fe}\left(\mathrm{TPPF}_{20} \beta-\mathrm{Cl}_{\mathrm{g}}\right) \mathrm{Cl}$ & Benzene & 4.5 & 243 & 28 & 56 & $(\mathrm{tr})$ & 15 \\
\hline \multirow[t]{3}{*}{$\mathrm{Cr}\left(\mathrm{TPPF}_{21} \beta-\mathrm{Cl} \mathrm{C}_{\mathrm{R}}\right) \mathrm{Cl}$} & Benzene & 5.5 & 9 & 40 & 60 & - & - \\
\hline & Benzene & 23.0 & 711 & 10 & 60 & 3 & 27 \\
\hline & Acetonitrile & 230 & 1518 & 7 & 48 & 5 & 40 \\
\hline \multirow[t]{2}{*}{$\mathrm{Cr}\left(\mathrm{TPPF}_{210} \beta-\mathrm{Cl}_{8}\right) \mathrm{N}_{3}$} & Benzene & 22 & 659 & 7 & 64 & 4 & 25 \\
\hline & Acetonitrile & 35 & 1508 & 12 & 51 & 3 & 34 \\
\hline
\end{tabular}


I able 10 Alkane Uridations Catalyzed by Iron IIalotetraphenylporphyrins"

Products mmole/ $100 \mathrm{~g}$ reaction mix

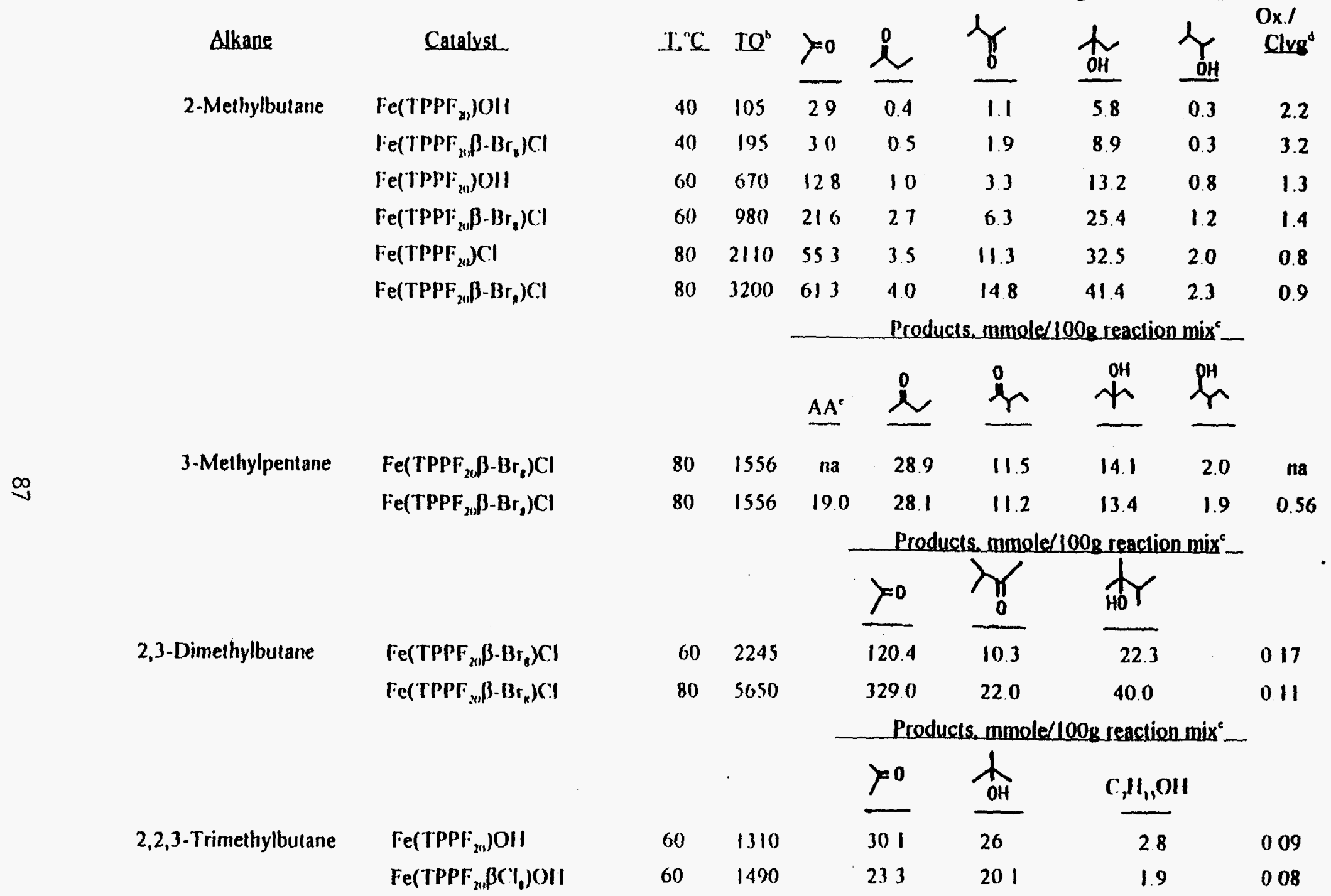

'Oxidations carried out by reacting oxygen with the neal alkane, $30 \mathrm{ml}$, containing 0.013 mmoles of the catalyst at $T$, ${ }^{\circ} \mathrm{C}$ at a total pressure of 100 psig for 6 hours. Product analysis by standardized GLPC "Moles $\mathrm{O}_{2}$ consumed/mole catalyst used. 'mmoles roduct 100 grams of recovered product mixture, by (il.PC' 'Ratio of moles ('-C. bond cleavage products/moles oxidation products with carbon skeleton inlact' 'AA=acetic acid. 
Table 11 Comparison of the Product Profile of 2-Methylpentane Oxidation with that of tern-Amyl Hydroperoxide Decomposition Catalyzed by Perhaloporphyrinato Iron Complexes

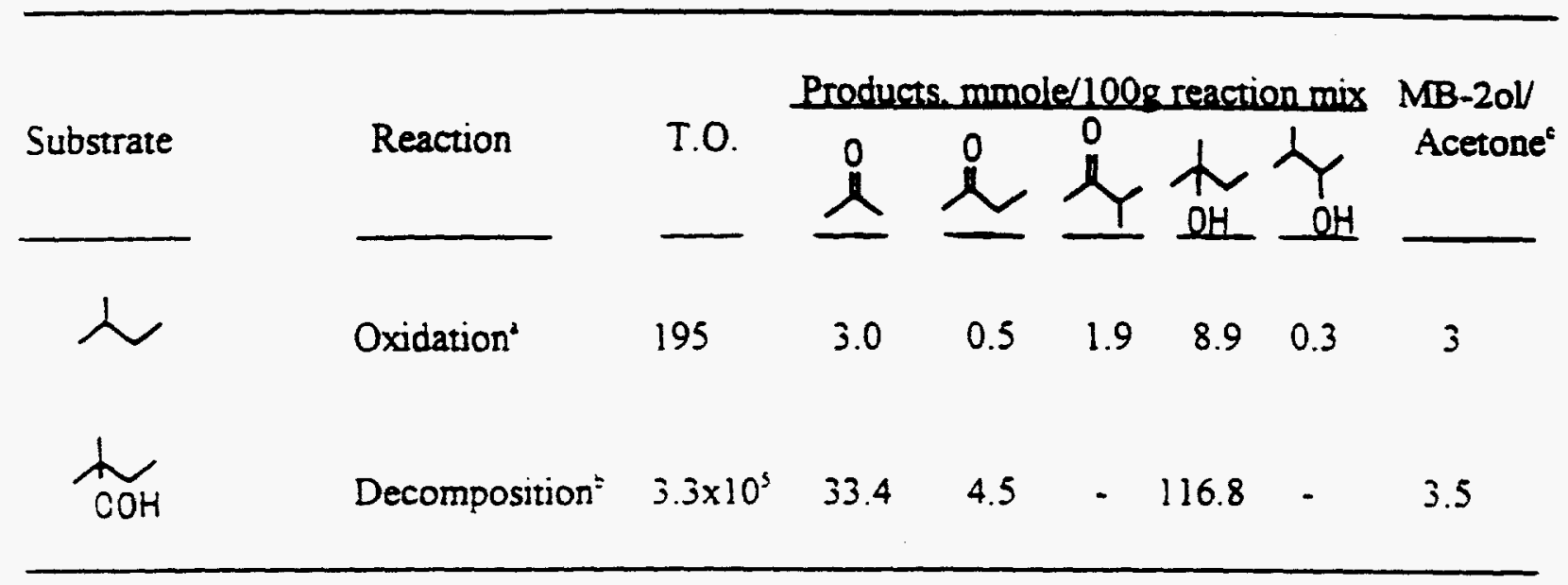

'Oxvgen is reacted with the neat alkane, $30 \mathrm{ml}$. containing 0.013 mmole of $\mathrm{Fe}\left(\mathrm{TPPF}_{20} \beta-\mathrm{Br}_{3}\right) \mathrm{OH}$ at $40^{\circ} \mathrm{C}$ at a total pressure of 100 psig for 6 hours. Product analysis by standardized GLPC. Ien-Amyl hydroperoxide, $10 \mathrm{ml}(82 \%)$, is added to a solution of $\mathrm{Fe}\left(\mathrm{TPPF}_{20} \mathrm{BCl}_{8}\right) \mathrm{Cl}, 4 \times 10^{-6} \mathrm{M}$, in benzene Oxygen evolution is followed manometrically and products are analyzed periodically by standanzed GLPC. The analysis reported in Table II was peformed after 1.9 hours.

$: 2-\mathrm{MBB}-2 \mathrm{ol} / \mathrm{Acetone}-\mathrm{the}$ ratio of 2-methylbutane-2-ol to acetone formed in the decomposition reaction. 
Table 12 Product Profile as a Function of $R$ in Oxidation of $R_{1} R_{2} R_{3} C H$ Catalyzed by Fe(TPPF 20 -Br8)Cl

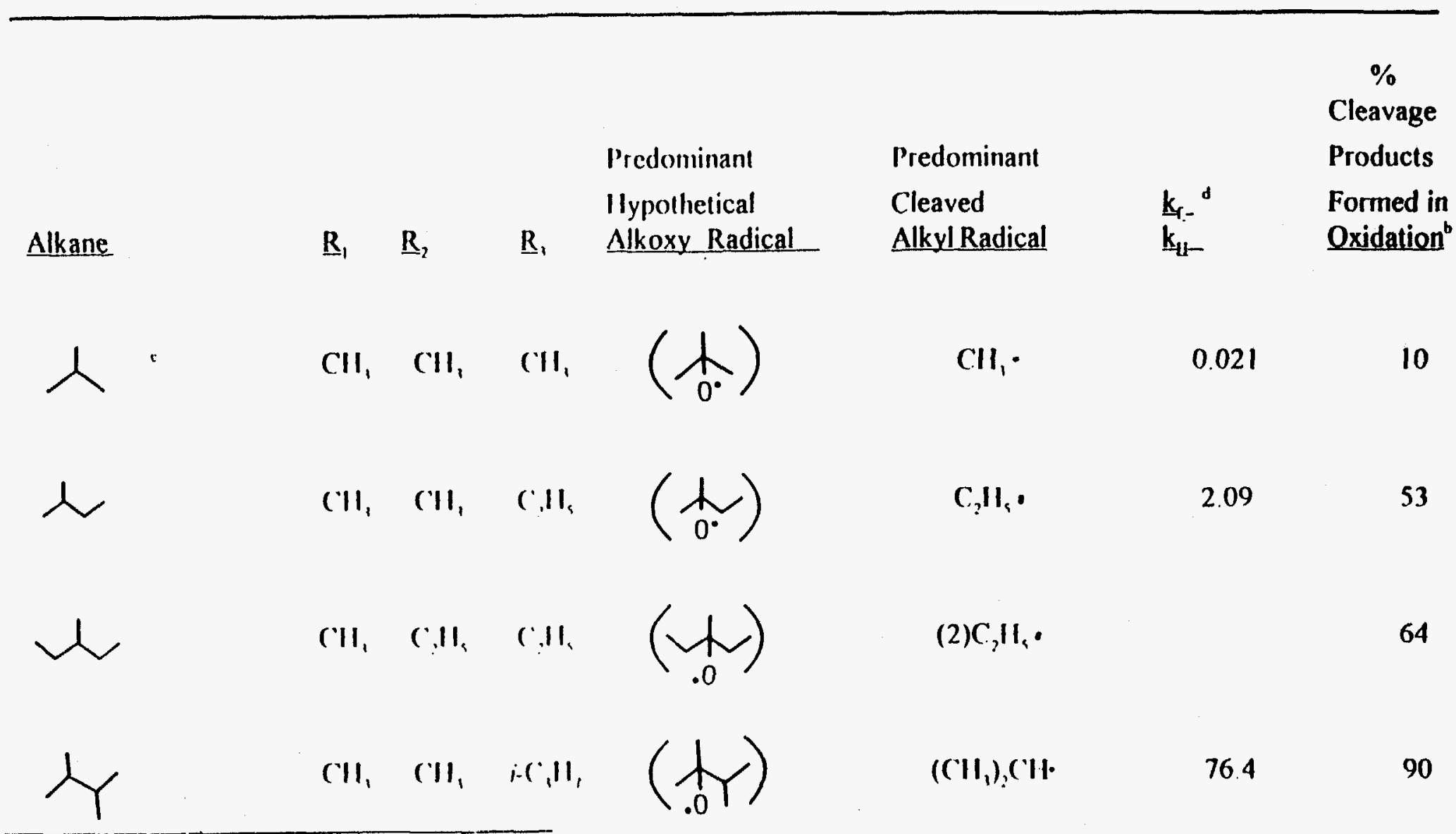

a) Oxidations carried out by reacting oxygen with the neat alkane, $30 \mathrm{ml}$, containing 0.013 mmoles of $\mathrm{Fe}\left(\mathrm{TPPF}_{20} \beta-\mathrm{Br}_{8}\right) \mathrm{Cl}$ at $80^{\circ} \mathrm{C}$ at a total pressure of 100 psig for 6 hours Product analysis by Standardized (il.PC' b)(Moles alkane converted to C-C bond cleavage products/moles alkane oxidized) $\times 100$ c) A 25 weiglı \% solution of isobutane in benzene was oxidized in this experiment. d) Ration of Rates of Alkyl (leavage and 11 -Abstraction fiom cyclohexane $(61)$ in the reaction

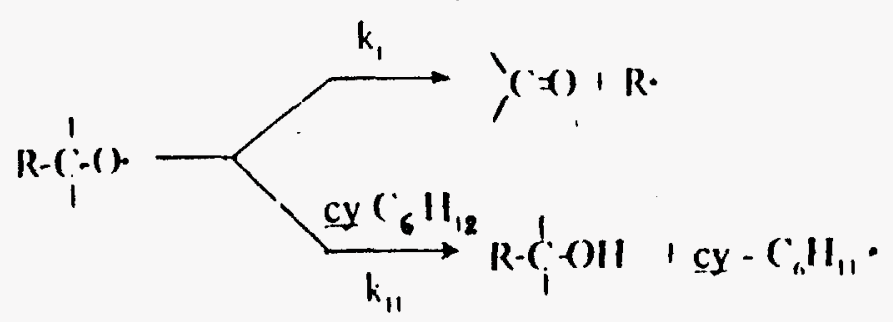


FIGURE 1 The Substituted Porphyrinato Ligand; $Y=\beta-C 1, B r, o r H ; Z=C_{6} \mathrm{H}_{5}(\mathrm{TPP}), \underline{0}-\mathrm{C}_{6} \mathrm{H}_{4} \mathrm{Cl}_{2}\left(\mathrm{TPPCl}_{8}\right)$ or $\mathrm{C}_{6} \mathrm{~F}_{5}\left(\mathrm{TPPF}_{20}\right)$.

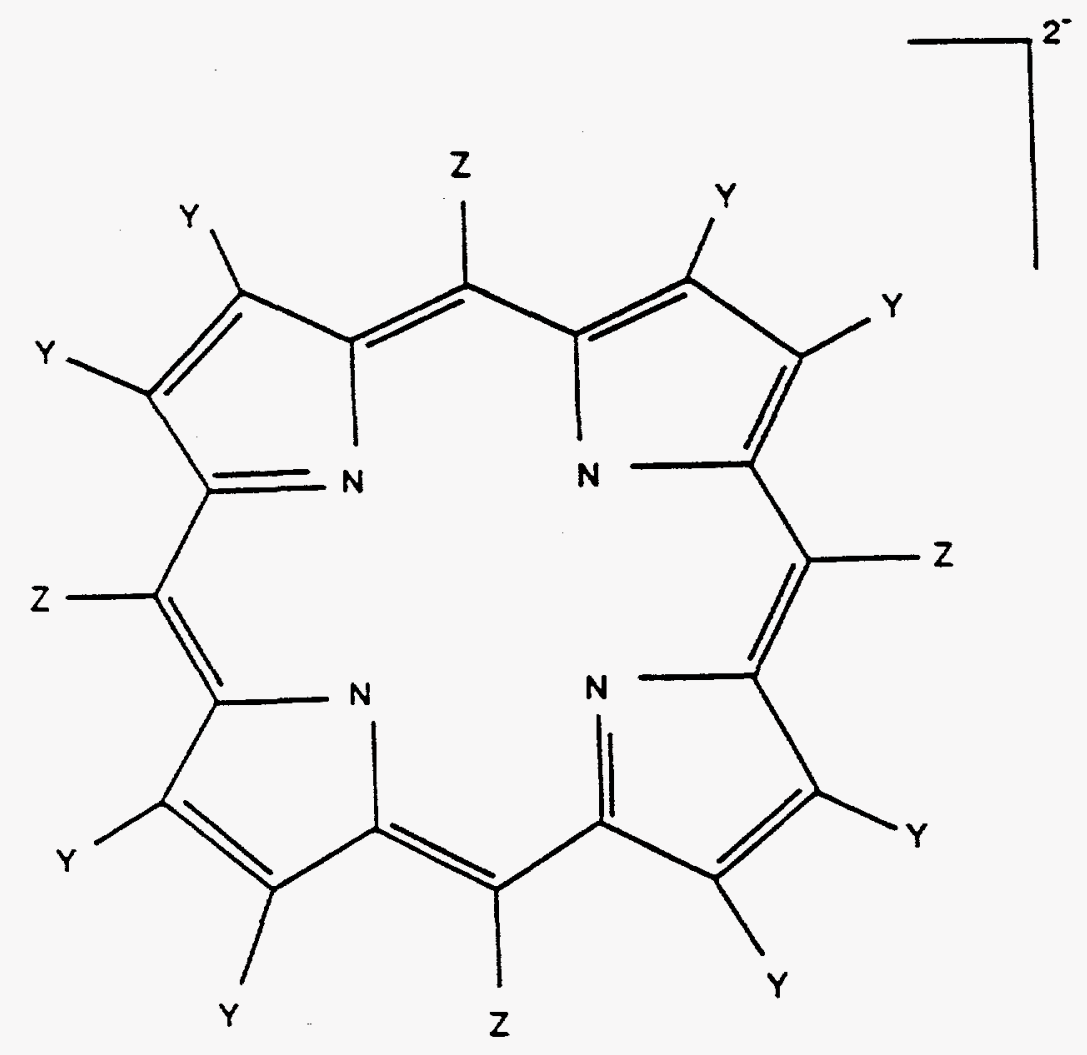


FIGURE 2 Oxidation of Isobutane in Benzene at $80^{\circ} \mathrm{C}$ using $\mathrm{Fe}\left(\mathrm{TPPF}_{20}\right) \mathrm{X} ; \mathrm{X}=\mathrm{Cl} \square$, $\mathrm{N}_{3}, \mathrm{O}$, and $\mathrm{OH}, \mathrm{O}$ as axial ligands.

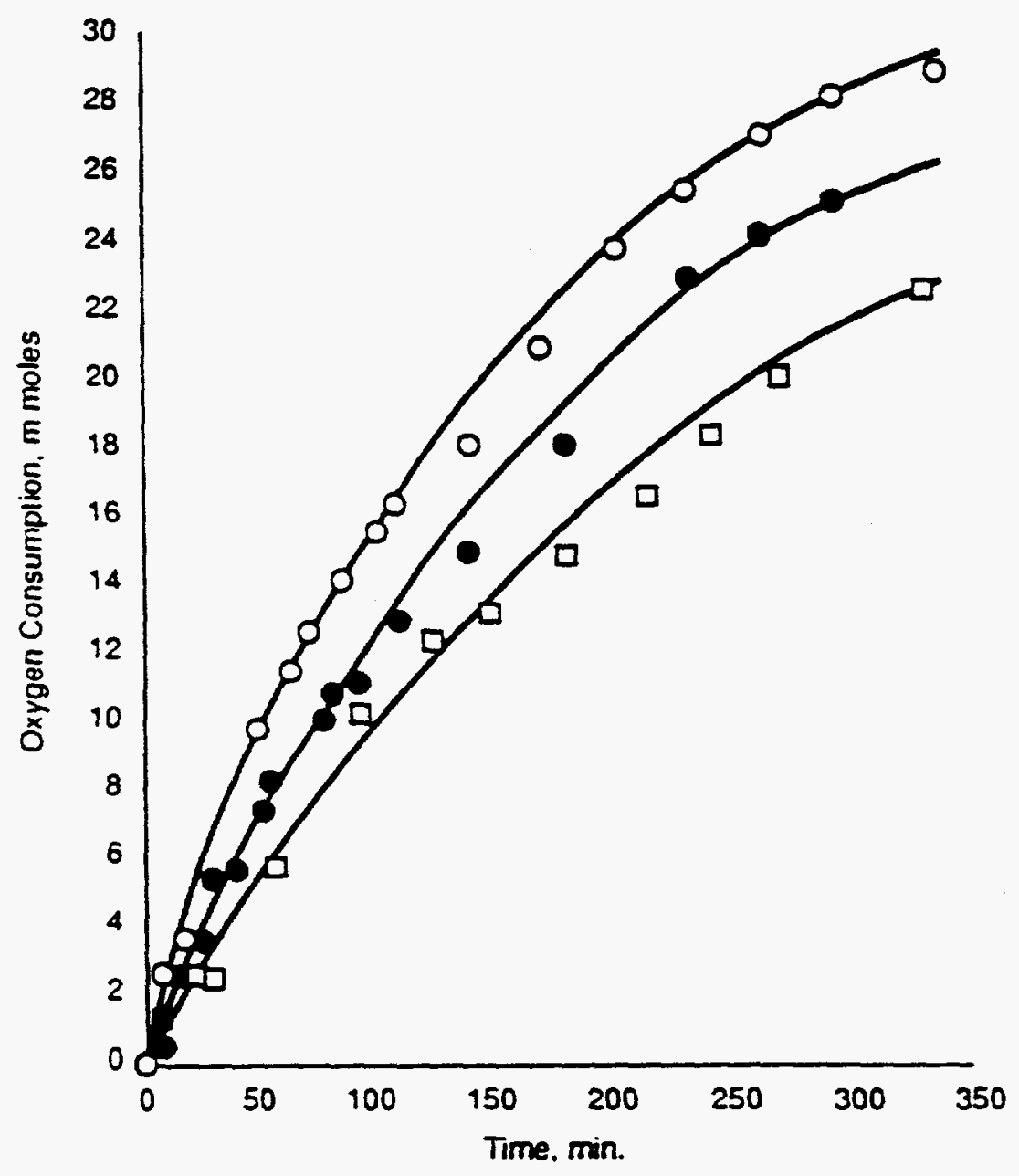


FIGURE 3 Catalyst Turnovers as a function of $\mathrm{Fe}(\mathrm{III}) / \mathrm{Fe}$ (II) Oxidation Potential. - - Isobutane Oxidations at $60^{\circ} \mathrm{C} ;-0$ - Propane Oxidations at $125^{\circ} \mathrm{C}$. See Tables 3,4 for experimental procedures. 1, Fe(TPP)OH; $2 \mathrm{Fe}\left(\mathrm{TPPCl}_{8} \mathrm{~B}-\mathrm{Br}_{4}\right) \mathrm{OH} ; 3, \mathrm{Fe}\left(\mathrm{TPPF}_{20}\right) \mathrm{OH} ; 4, \mathrm{Fe}\left(\mathrm{TPPF}_{20} \mathrm{\beta}\right.$ -

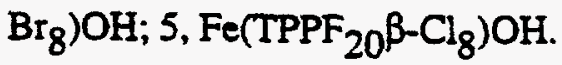

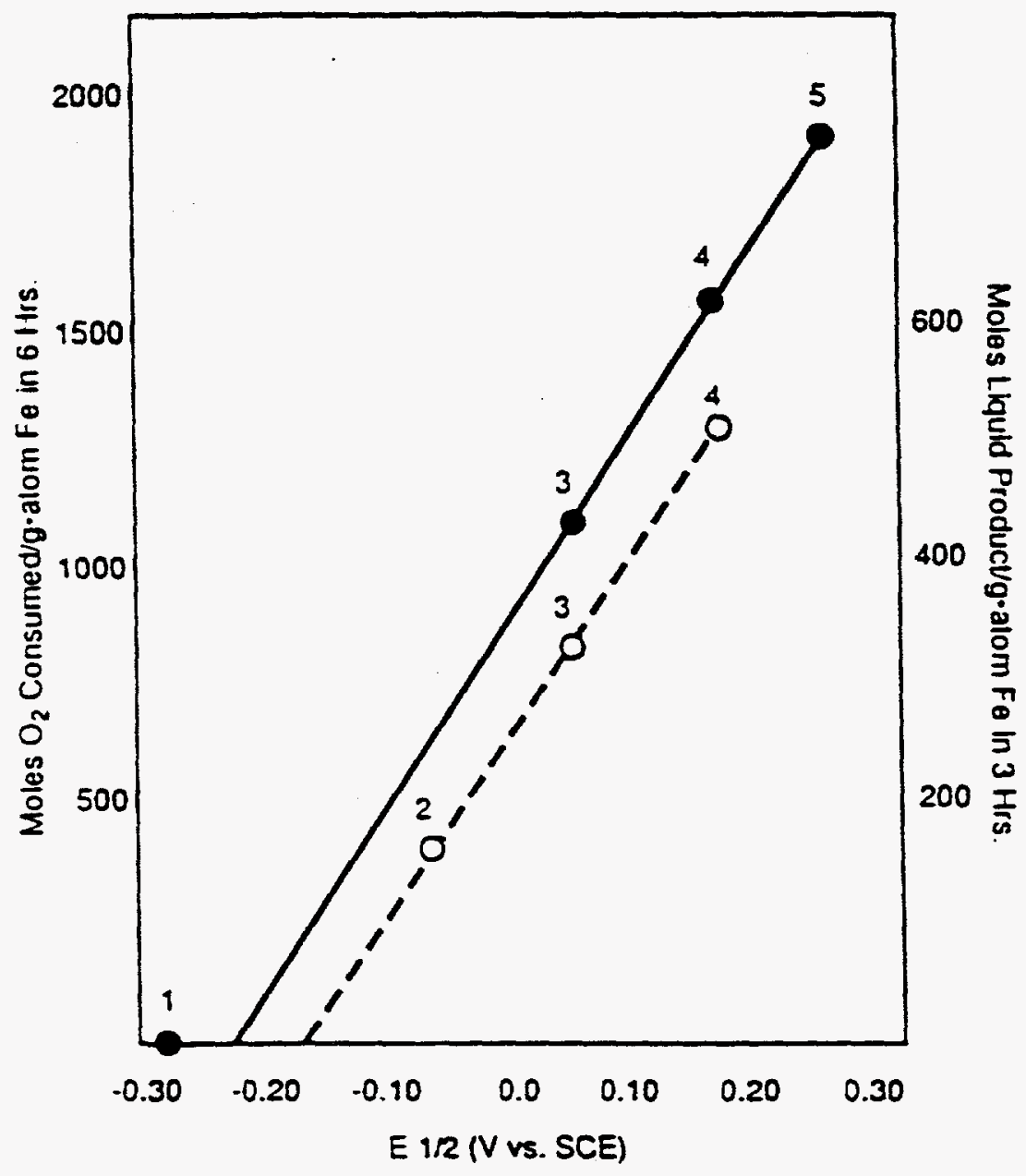


FIGURE 4 Schematic representation of biological and biomimetic alkane oxidation (40-47)

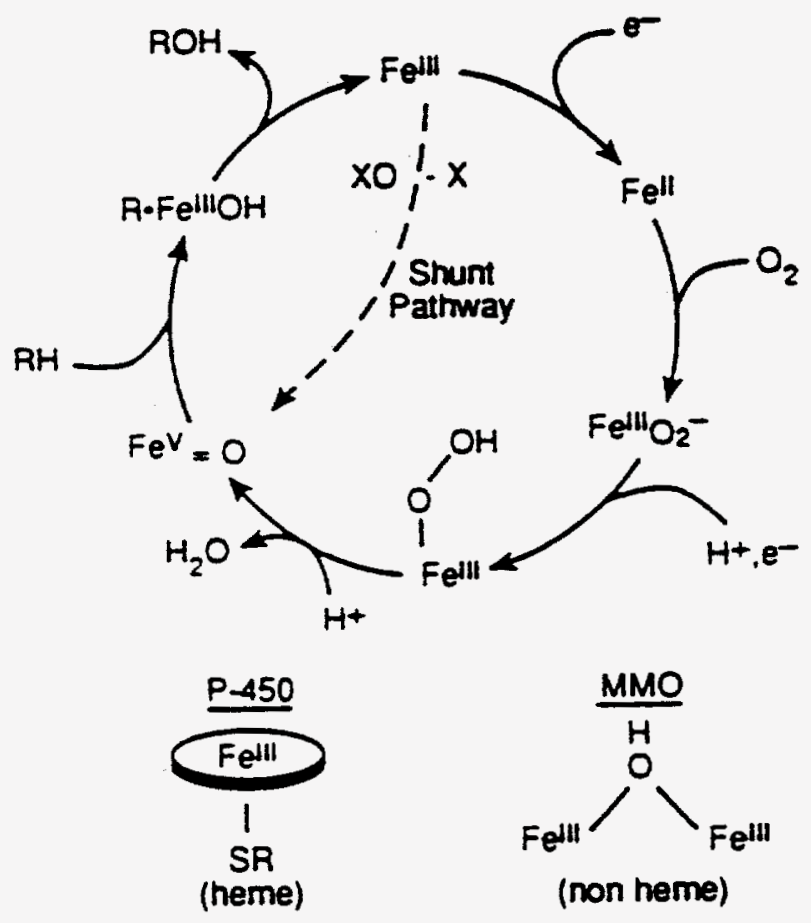


FIGURE 5 Conceptual scheme for a hypothetical catalytic cycle for conversion of an aikane to an alcohol using an electron deficient iron porphyrin complex (porphyrin ring omitted).

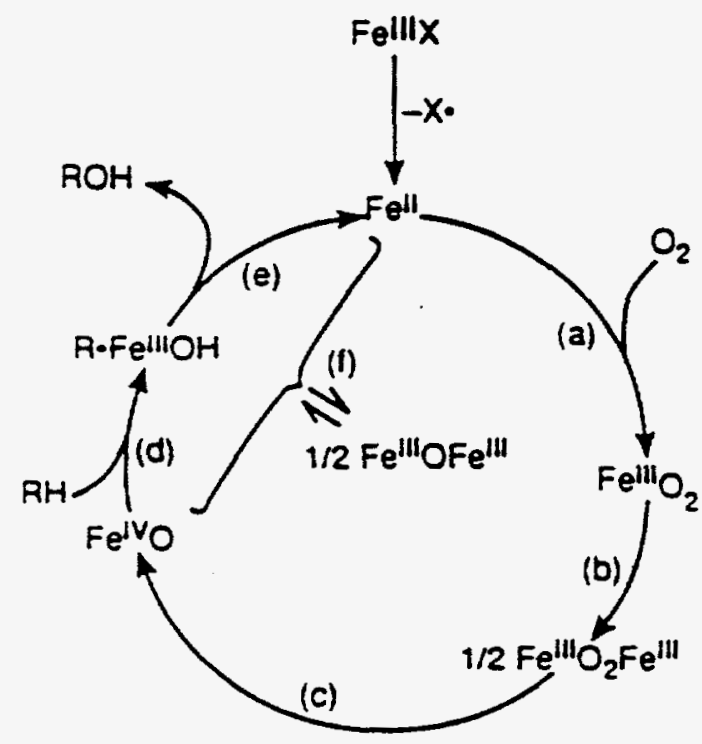


FGURE 6 Formation of tert-buryl alcohol, TBA, from tert-buryl hydroperoxide $(10 \mathrm{ml})$ in benzene $(48 \mathrm{ml})$-p-xylene $(2.4 \mathrm{ml})$ using $2 \times 10-4$ mmoles of catalyst: $O$ $\mathrm{Fe}\left(\mathrm{TPPF}_{20} \mathrm{\beta}-\mathrm{Cl}_{8}\right) \mathrm{Cl}, \square \mathrm{Fe}\left(\mathrm{TPPF}_{20}\right) \mathrm{Cl}$, $\mathrm{Fe}(\mathrm{TPP}) \mathrm{Cl}, \mathrm{Fe}(\mathrm{acac}) 3$.

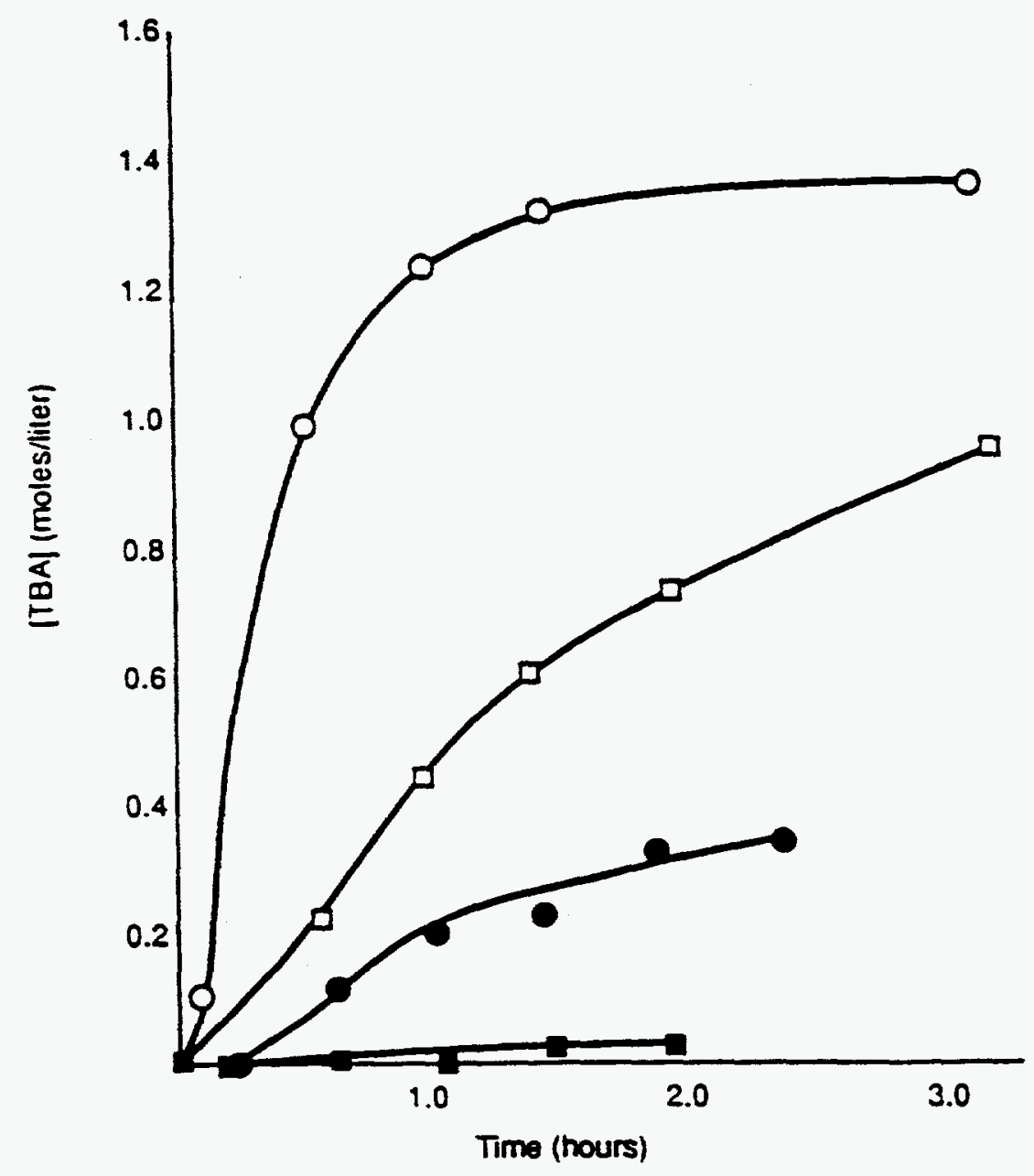


FIGURE 7 Haber-Weiss decomposition of hydroperoxides using metalloporphyrins as catalysts (porphyrin ring omitted).

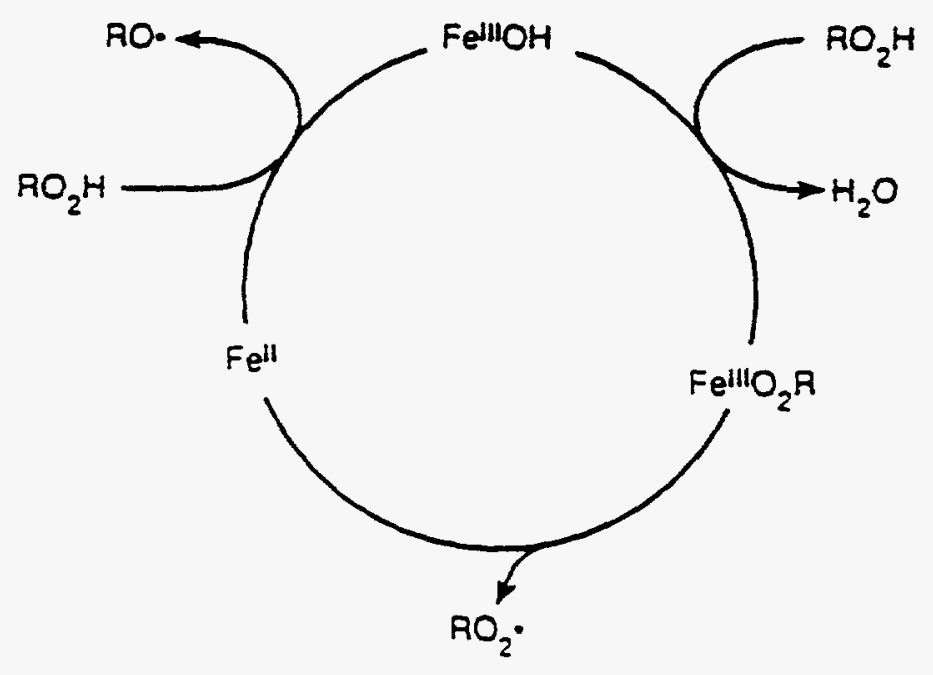

$$
2 \mathrm{RO}_{2} \mathrm{H} \longrightarrow \mathrm{RO}_{2} \cdot+\mathrm{RO} \cdot+\mathrm{H}_{2} \mathrm{O}
$$

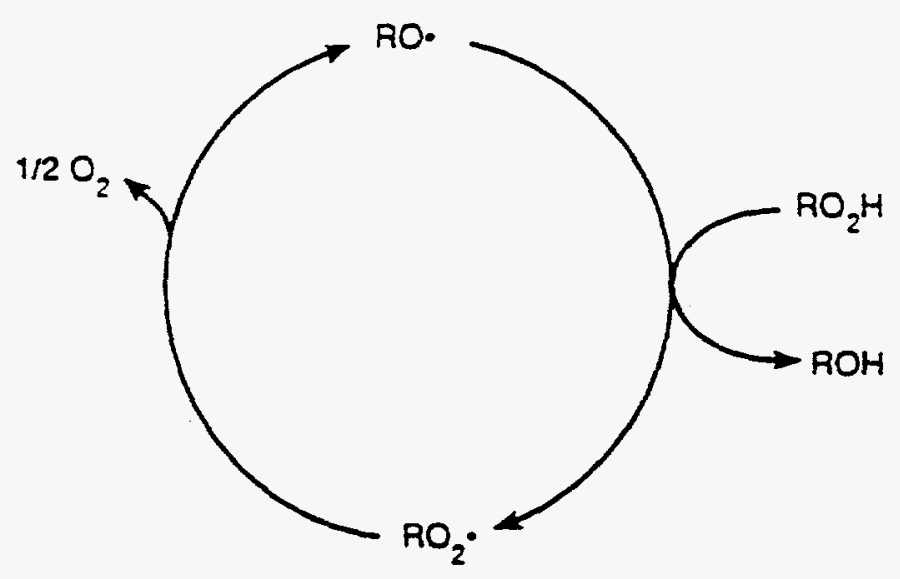


FGGURE 8 Oxidation of $n$-butane in acetonitrile at $125^{\circ} \mathrm{C}$ catalyzed by $\operatorname{Cr}\left(\operatorname{TPPF}_{20} \beta-\mathrm{Cl}_{8}\right) \mathrm{N}_{3}$. Details given in Table 10.

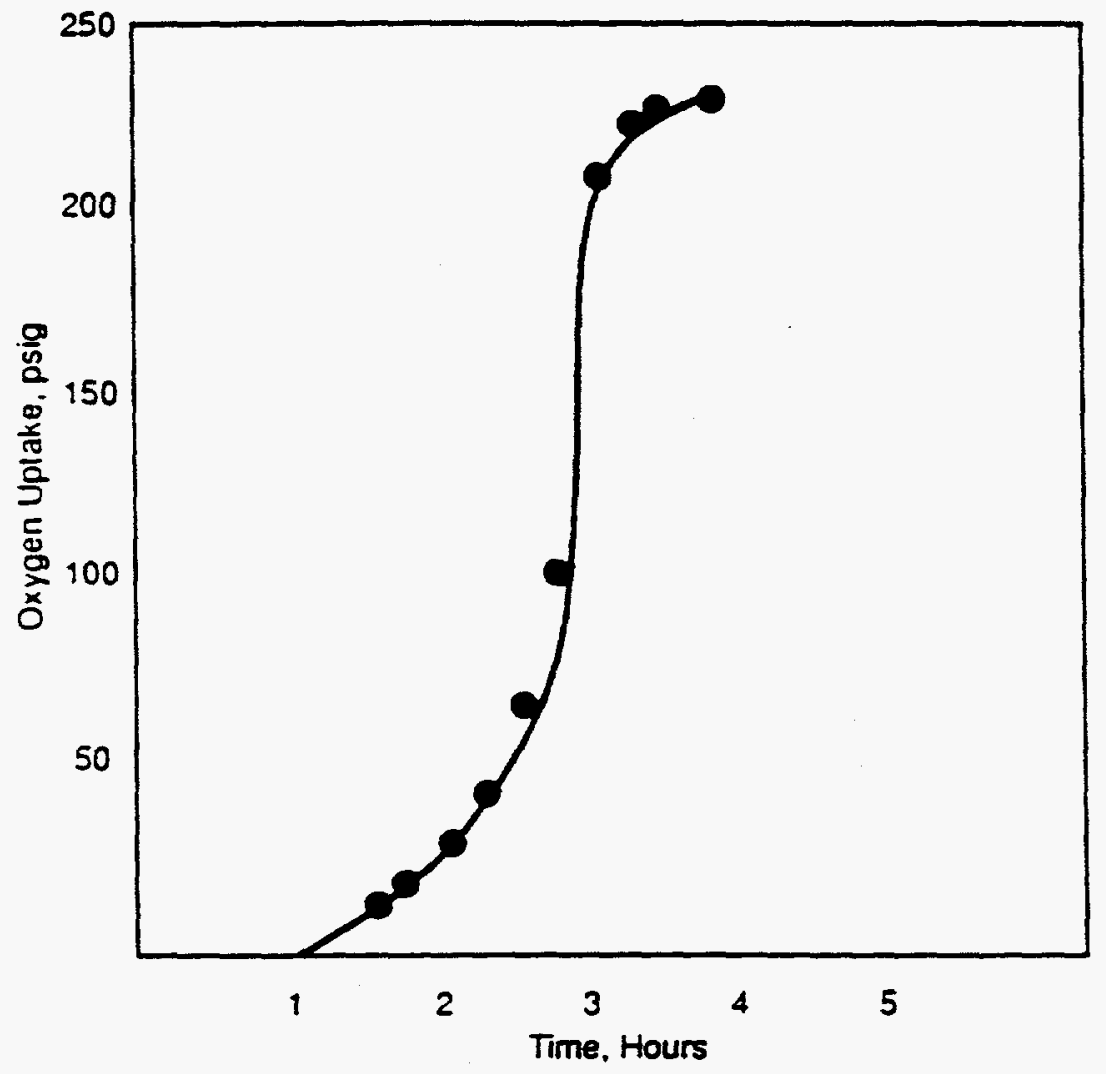


FGURE 9 Dependence of product profile on substrate structure for alkane oxidations catalyzed by $\mathrm{Fe}\left(\mathrm{TPPF}_{20} \mathrm{\beta}-\mathrm{Br}_{8}\right) \mathrm{OH}$.

Substrate Alkoxy Radicala Oxygenations $\begin{array}{r}\text { Oxidative } \\ \text { Cleavage }\end{array}$

1.

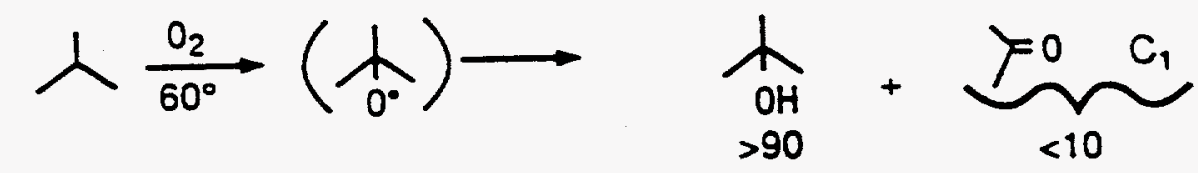

2.

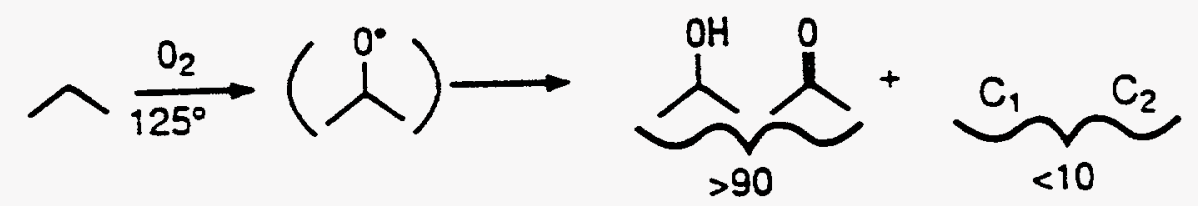

3.

$\sim \frac{\mathrm{O}_{2}}{125^{\circ}}\left(\stackrel{0^{\circ}}{\sim}\right) \longrightarrow$<smiles>CCCC(CC)(CCC)CC(=O)CC</smiles><smiles>CCCCCCCCCC(C)OC(C)O</smiles>

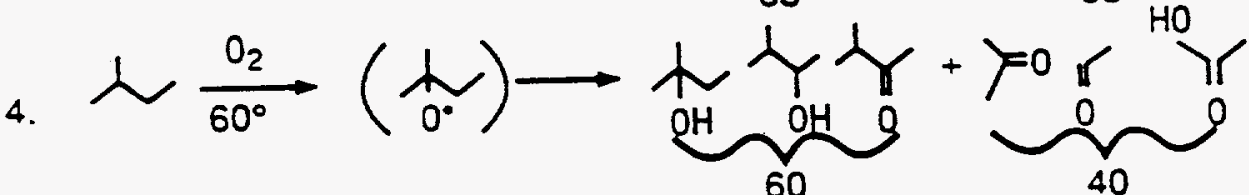

5.

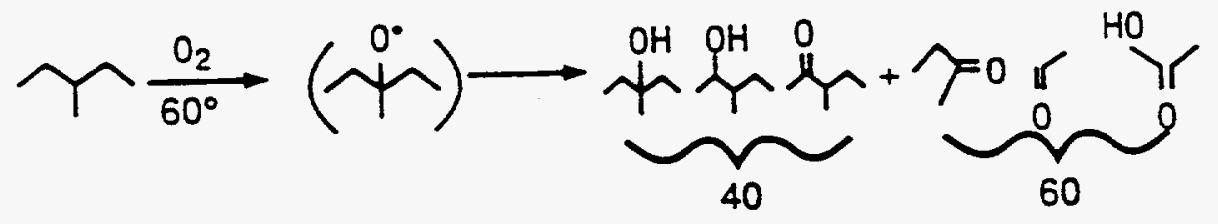

6.

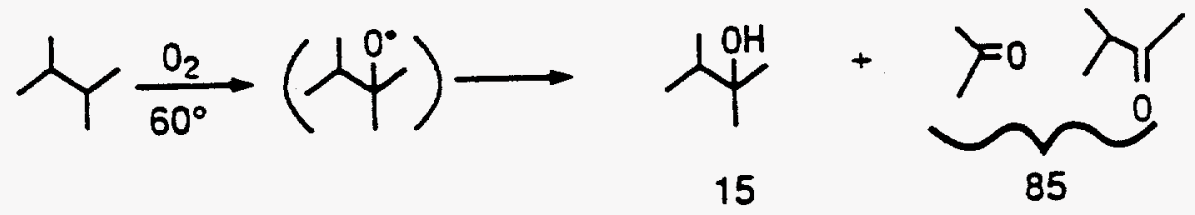

7. $K \frac{\mathrm{O}_{2}}{60^{\circ}}\left(\mathrm{HO}^{\circ}\right) \longrightarrow$

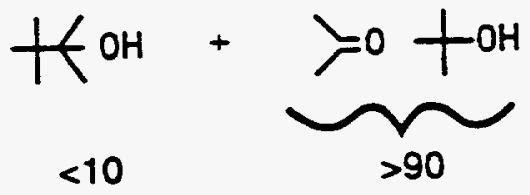


FIGURE 10 Possible pathways occuring during selective oxidation of alkanes catalyzed by iron perhaloporphyrin complexes. a) hypothetical alkoxy radical.
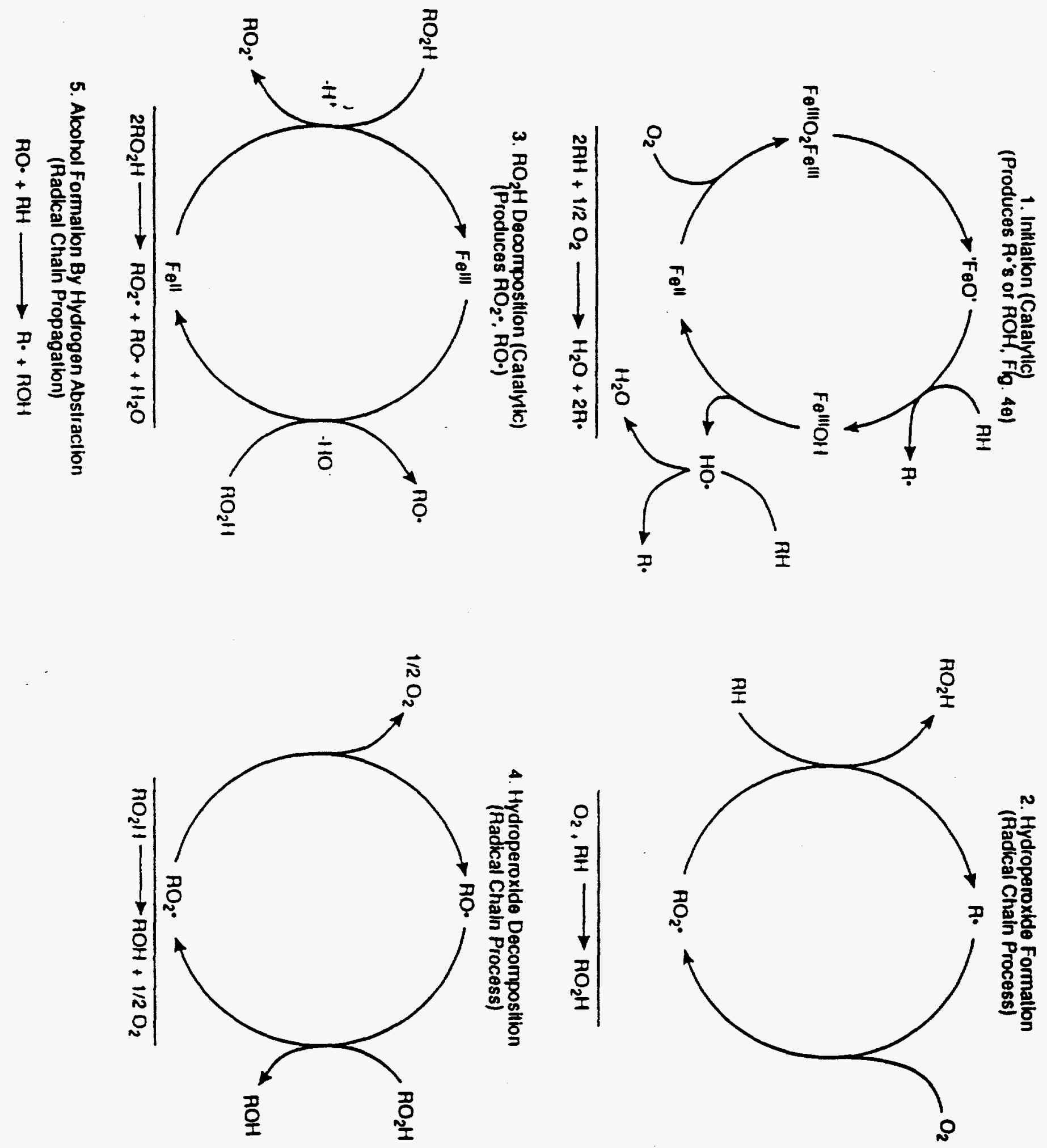


\subsection{PERFLUOROALKYLPORPHYRIN COMPLEXES AS ACTIVE CATALYSTS FOR THE BEACTION OF ISOBUTANE WITH OXYGEN AND THE DECOMPOSTTION OF tert-BUTYILHYDROPEROXIDE}

\subsection{ABSTRACT}

A new family of metalloporphyrin complexes having perfluoroalkyl groups in the mesoposition of the ring are active catalysts for the oxidation of isobutane to tert-butyl alcohol, TBA, and for the decomposition of tert-butyl hydroperoxide to TBA. This discovery extends the limited number of meso-substituents that can be used to enhance catalytic activity and fits the postulate that groups that withdraw electrons from the porphyrin periphery give rise to active catalysts for alkane oxidation. The perfluoroalkyl groups also confer oxidative stability, hydrophobicity and lower cost to the catalyst.

Keywords - Oxidation, Metalloporphyrins, Hydroperoxide Decomposition

\subsection{INTRODUCTION}

It has recently been shown that electron withdrawing substituents such as perfluorophenyl and nitro groups in the meso-position of iron porphyrin complexes have an enormously beneficial effect on the catalytic activity of these complexes for both the direct reaction of aliphatic hydrocarbons with molecular oxygen as well as the rapid and selective decomposition of alkyl hydroperoxides to the corresponding alcohols (1-5). This enhanced catalytic activity can be correlated directly-with increased $\mathrm{Fe}(\mathrm{III}) / \mathrm{Fe}(\mathrm{II})$ reduction potentials of the electron deficient complexes in question (6).

A novel series of electron-deficient porphyrin complexes has recently been prepared in our laboratories (7) by a new and general synthetic route involving 5-perfluoroalkyldipyrromethanes as the key intermediates (see Section 9). This route allows us to prepare for the first time, complexes having perfluoroalkyl groups at opposite $(5,15-)$ meso-positions while the substituents at the other two meso- $(10,20-)$ positions can varied as desired, Figure 1. This method furnishes a convenient high yield synthesis of 5,10,15,20-tetrakisperfluoroalkyl porphyrin complexes (7). We are now in a position to examine the effect of a mese-perfluoroalkyl substituent on the catalytic activity of a metalloporphyrin catalyst and to compare this effect with that of either the meso-nitro or meso-pentafluorophenyl groups which we have examined earlier.

Not only does this new route make available a series of potentially inexpensive perhaloporphyrin catalysts but also provides a family of truly "teflon" porphyrins having pendant perfluoroalkyl chains which might be both more robust and more highly hydrophobic $(7,8)$ than any currently available. Moreover, perfluoroalkyl substituents, though highly electron withdrawing, is chemically distinct from the two types that we have investigated in the past. Past substituents included haloaryl groups such as meso- $\mathrm{C}_{6} \mathrm{~F}_{5}$ and the highly electron-withdrawing meso-nitro group both of which might participate in electron withdrawal through $\pi$-interactions with the delocallized $\pi$-system of the porphyrin ring. The perfluoroalkyl substituent, on the other hand can only withdraw electrons from the porphyrin $\pi$-system via a sigma-inductive interaction. Whether this type of interaction will provide a superior catalyst has been up until now an interesting question. In this communication we 
demonstrate for the first time that perfluoroalkyl groups in the meso-position of iron porphyrin complexes result in highly active catalysts for the oxidation of alkanes and the decomposition of alkyl hydroperoxides.

\subsection{EXPERMMENTAL}

\section{Solvents and Reagents}

All solvents used were HPLC grade from Aldrich and were used as purchased. All non-porphyrin reagents were purchased from Aldrich unless noted and were certified ACS reagent grade or better. $\left.\left[\mathrm{C}_{6} \mathrm{H}_{5}\right)_{4} \mathrm{PFe}\right] \mathrm{Cl}$, I, and $\left[\left(\mathrm{C}_{6} \mathrm{H}_{5}\right)_{4} \mathrm{PFe}\right]_{2} \mathrm{O}$, II, were purchased from Strem Chemicals. $\left[\left(\mathrm{C}_{6} \mathrm{~F}_{5}\right)_{4} \mathrm{PFe}\right] \mathrm{OH}, \mathrm{III},(9)$ and $\left[\left(\mathrm{C}_{6} \mathrm{~F}_{5}\right)_{4} \quad \mathrm{~B}-\mathrm{Br}_{4} \mathrm{PFe}\right] \mathrm{OH},(10)$ were made by the referenced methods. $\left[\left(\mathrm{C}_{6} \mathrm{~F}_{5}\right)_{4} \mathrm{P}-\mathrm{Br}_{8} \mathrm{PFe}\right] \mathrm{OHIV}$, was prepared by chromatographic elution of the corresponding chloride complex on activated alumina. The syntheses of the meso-perhaloalkylporphyrins used to prepare the catalysts for this study, $\left(\mathrm{C}_{6} \mathrm{~F}_{5}\right)_{2}\left(\mathrm{CF}_{3}\right)_{4} \mathrm{PH}_{2},\left(\mathrm{CF}_{3}\right)_{4} \mathrm{PH}_{2},\left(\mathrm{C}_{6} \mathrm{~F}_{5}\right)_{2}\left(\mathrm{C}_{3} \mathrm{~F}_{7}\right) \mathrm{PH}_{2}$, and $\left(\mathrm{C}_{3} \mathrm{~F}_{7}\right)_{4} \mathrm{PH}_{2}$ are reported elsewhere (7). The syntheses of $\left[5,15-\left(\mathrm{C}_{6} \mathrm{~F}_{5}\right)_{2}-10,20-\left(\mathrm{CF}_{3}\right)_{2} \mathrm{PFe}\right]_{2} \mathrm{O}, \mathrm{V},\left[\left(\mathrm{CF}_{3}\right)_{4} \mathrm{PFe}\right] \mathrm{OH}, \mathrm{VI}$, $\left[5,15-\left(\mathrm{C}_{6} \mathrm{~F}_{5}\right)_{2}-10,20-\left(\mathrm{C}_{3} \mathrm{~F}_{7}\right)_{2} \mathrm{PFe}\right]_{2} \mathrm{O}$, VII and $\left[\left(\mathrm{C}_{3} \mathrm{~F}_{7}\right)_{4} \mathrm{PFe}\right] \mathrm{OH}$, VIII were accomplished by iron insetion into the porphyrin macrocycle as described below.

Iron insertion was carried out in hot glacial acetic acid $\left(110^{\circ} \mathrm{C}\right)$ under an inert atmosphere using $\mathrm{FeCl}_{2} \cdot 4 \mathrm{H}_{2} \mathrm{O}$. The reaction mixture was cooled to room temperature after $25 \mathrm{~min}$, allowed to stir overnight exposed to air and treated with an equal volume of $3 \mathrm{M} \mathrm{HCl}$. In each case, the curde product was isolated by filtration, chromatographed on neutral alumina and retreated with $6 \mathrm{M} \mathrm{HCl}$ to obtain the hemin [iron(III)chloride] in $>90 \%$ yield.

Analytical Data for the Hemins [Iron(III) Chloride]

\begin{tabular}{|c|c|c|c|}
\hline Complex & $\mathrm{UV}\left(\lambda_{\mathrm{max}}\right) \mathrm{nm}$ & $\begin{array}{c}300 \mathrm{MHz} \\
{ }^{1} \mathrm{H} \text { NMR } \\
\left(\delta_{\mathrm{CDC13}}\right)\left(\beta_{H}\right)\end{array}$ & $\operatorname{MS}(\mathbf{m} / x)$ \\
\hline$\left(\mathrm{C}_{6} \mathrm{~F}_{5}\right)_{2}\left(\mathrm{CF}_{3}\right)_{2} \mathrm{PFeCl}$ & 348/406 (Soret),504,622(wk) & $85.62,89.51$ & EIMS:832(M-Cl) \\
\hline$\left(\mathrm{CF}_{3}\right)_{4} \mathrm{PFeCl}$ & $348 / 404$ (Soret),506,634(wk) & 93.38 & EMMS:636 (M-Cl) \\
\hline$\left(\mathrm{C}_{6} \mathrm{~F}_{5}\right)_{2}\left(\mathrm{C}_{3} \mathrm{~F}_{7}\right)_{2} \mathrm{PFeCl}$ & $348 / 406$ (Soret),510,622 (wk) & $83.39,86.05,87.45,88.86$ & FABMS:1032(M-Cl) \\
\hline$\left(\mathrm{C}_{3} \mathrm{~F}_{7}\right)_{4} \mathrm{PFeCl}$ & $348 / 406$ (Soret), 506,630 (wk) & $84.86,96.20,99.98$ & LDMS: $1037(\mathrm{M}-\mathrm{CH}+\mathrm{H})$ \\
\hline
\end{tabular}


The catalytic species were prepared by passing a $\mathrm{CHCl}_{3}$ solution through deactivated alumina (neutral; $15 \%$ water added) using $\mathrm{CHCl}_{3}$ for elution. ${ }^{1} \mathrm{HNMR}\left(\delta_{\mathrm{CDCl} 13}\right)$ indicate that they are $\mu$-oxo dimers: $\left[\left(\mathrm{C}_{6} \mathrm{~F}_{5}\right)_{2}\left(\mathrm{CF}_{3}\right)_{2} \mathrm{PFe}\right]_{2} \mathrm{O}\left(\beta_{\mathrm{H}}\right.$ at $\left.14.22,14.49 \mathrm{ppm}\right),\left[\left(\mathrm{C}_{6} \mathrm{~F}_{5}\right)_{2}\left(\mathrm{C}_{3} \mathrm{~F}_{7}\right)_{2} \mathrm{PFe}\right]_{2} \mathrm{O}\left(\beta_{\mathrm{H}}\right.$ at $\left.14.06,14.45 \mathrm{ppm}\right)$ and $\left[\left(\mathrm{C}_{3} \mathrm{~F}_{7}\right)_{4} \mathrm{PFe}\right]_{2} \mathrm{O}\left(\beta_{\mathrm{H}}\right.$ at $\left.14.45 \mathrm{ppm}\right)$. In the case of $\left(\mathrm{CF}_{3}\right)_{4} \mathrm{PFe}$, extensive molecular aggregation resulted in the loss of significant quantities of material on the column, and the dimer $\left[\left(\mathrm{CF}_{3}\right)_{4} \mathrm{PFe}\right]_{2} \mathrm{O}$ isolated was highly insoluble in most organic solvents.

\section{$\beta$ - Bromination of \\ 5,15-bis(pentafluorophenyl)-10,20-bis(trifluoromethyl)porphyrin}

The brominated complexes: $\left[5,15-\left(\mathrm{C}_{6} \mathrm{~F}_{5}\right)_{2}-10,20-\left(\mathrm{CF}_{3}\right)_{2} \beta-\mathrm{Br}_{x} \mathrm{PFe}\right]_{\mathrm{y}} \mathrm{X}, \mathrm{x}=1-7, \mathrm{y}=1$ or $2 ; \mathrm{X}=\mathrm{OH}$ and/or $\mu-\mathrm{O}, \mathrm{V}-\mathrm{Vd}$, were prepared by direct bromination of $\mathrm{V}$. $\beta$-Bromination was carried out on the iron (III) chloride $\left[\left(\mathrm{C}_{6} \mathrm{~F}_{5}\right)_{2}\left(\mathrm{CF}_{3}\right)_{2} \mathrm{PFe}\right] \mathrm{Cl}$ using four different procedures described below. In each case, the reaction mixture was treated with $2 \mathrm{M} \mathrm{NaOH}$ and chromatographed on neutral alumina (15\% water added) prior to isolation of the product. This was to ensure that the catalyst is either in the hydroxoor $\mu$-oxo dimer form depending on the extent of $\beta$-bromination (determined by FABMS).

1. $\left[\left(\mathrm{C}_{6} \mathrm{~F}_{5}\right)_{2}\left(\mathrm{CF}_{3}\right)_{2} \mathrm{PFe}\right] \mathrm{Cl}$, was refluxed with bromine for $3 \mathrm{Oh}$. The isolated material exhibited a $\mu$-oxo dimer type uv-visible spectrum with a Soret at $\lambda_{\max }=396 \mathrm{~nm}(10 \mathrm{~nm}$ red-shift from that of $\left[\left(\mathrm{C}_{6} \mathrm{~F}_{5}\right)_{2}\left(\mathrm{CF}_{3}\right)_{2} \mathrm{PFe}\right]_{2} \mathrm{O}$ and a shoulder at $430 \mathrm{~nm}$. The predominant species: $\left[\left(\mathrm{C}_{6} \mathrm{~F}_{5}\right)_{2}\left(\mathrm{CF}_{3}\right)_{2} \mathrm{\beta}-\mathrm{Br} \mathrm{PFe}_{3} \mathrm{PH}\right.$ and dimers of mono-and di-brominated material, $(\mathrm{Va})$

2. Bromination as in 1 above but with added N-bromosuccinimide. Product exhibited a Soret at $\lambda_{\max }=406 \mathrm{~nm}$ with peaks at $446(\mathrm{sh})$ and $640(\mathrm{wk})$. The predominant species:

$\left[\left(\mathrm{C}_{6} \mathrm{~F}_{5}\right)_{2}\left(\mathrm{CF}_{3}\right)_{2} \beta-\mathrm{Br}_{4} \mathrm{PFe}\right] \mathrm{OH},(\mathrm{Vb})$.

3. $\left[\left(\mathrm{C}_{6} \mathrm{~F}_{5}\right)_{2}\left(\mathrm{CF}_{3}\right)_{2} \mathrm{PFe}\right] \mathrm{Cl}$ in $\mathrm{CCl}_{4}$ /pyridine (20:1) was refluxed with bromine and $\mathrm{N}$-bromosuccinimide for $24 \mathrm{~h}$. The supernatant solution was decanted from insoluble material and washed with $\mathrm{HCl}$ to remove excess pyridine prior to the standard work-up. The isolated material exhibited a broad Soret at $\lambda_{\max }=407 \mathrm{~nm}$ with a peak at $655(\mathrm{wk})$. The predominant species: monomeric penta- and hexa-brominated material, ( $\mathrm{Vc})$.

4. Bromination as in 3 above with no added $\mathrm{N}$-bromosuccinimide. The reaction mixture exhibited a $30 \mathrm{~nm}$ red shift of the Soret band within $1 \mathrm{~h}$ with no further change even in $7 \mathrm{~h}$. The isolated material exhibited a very broad Soret at $\lambda_{\max }=418 \mathrm{~nm}$ and a peak at 685 (wk). The predominant species: monomeric hexa-; hepta- and octa-brominated material, (Vd). 


\section{Isobutane Oxidations}

Isobutane was oxidized in the liquid phase according to the procedures given in Tables 1 and 2. Glass vessels were used and many of the reactions were carried out within the explosive region. For this reason reactors were individually shielded within a laboratory hood and the entire hood front was protected by $1.4^{\prime \prime}$ thick polycarbonate shielding.

\section{tert-Butylhydroperoxide Decompositions}

tert-Butylhydroperoxide was decomposed under the conditions given in Table 1. Reactions were carried out in magnetically stirred Morton flasks immersed in a constant temperature bath kept at $80^{\circ}$ $\mathrm{C} \pm 1^{\circ} \mathrm{C}$ during reaction. When the reaction mixture reached $80^{\circ} \mathrm{C}$ the catalyst was added in a small glass boat to the stirring solution. Oxygen immediately began to evolve. The reflux condenser was connected to a manometer and oxygen evolution measured with time. Liquid samples were removed periodically through an "air-tight" port and subjected to standardized glpc analysis. The reactor hood opening was protected with polycarbonate shielding as in the isobutane oxidations above.

\subsection{RESULTS AND DISCUSSION}

Table 1 indicates that iron porphyrin complexes having perfluoroalkyl groups in the mesoposition are active catalysts for the selective oxidation of isobutane to tert-butyl alcohol,eq. 1 . New complexes tested were iron haloporphyrin complexes having either hydroxo or $\mu$-oxo axial ligands in all cases. It can be seen that replacing two of the perhalophenyl groups of meso-5, 10,15,20-tetrakis(pentafluorophenyl)porphyrinatoiron(III) hydroxide, III, with perhalomethyl substituents results in a catalyst, $\mathrm{V}$, with fair activity.

$$
\left(\mathrm{CH}_{3}\right)_{3} \mathrm{CH}+1 / 2 \mathrm{O}_{2} \longrightarrow\left(\mathrm{CH}_{3}\right)_{3} \mathrm{COH}
$$

It was of considerable interest, therefore, to prepare the analogous complex in which all four of the meso-substituents were $\mathrm{CF}_{3}$ groups to determine its catalytic activity. In addition there are economic incentives for studying the activity of tetra(meso-trifluoromethyl) substituted iron porphyrin complexes as replacements for the tetra(meso-pentafluorophenyl) substituted complexes. One is that the perhalogenated precursors used for the introduction of meso- $\mathrm{CF}_{3}$ groups are less expensive than those required for introducing meso $-\mathrm{C}_{6} \mathrm{~F}_{5}$ groups. Another is that the substitution of four $\mathrm{CF}_{3}$ groups for four $C_{6} F_{5}$ groups lowers the weight of catalyst required per active iron, thus lowering catalyst cost directly. We, therefore, prepared the 5,10,15,20-tetrakis(trifluoromethyl)iron(III) complex, VI, with the intention of using this complex as an isobutane oxidation catalyst. It was, unfortunately, insoluble in all nonpolar aprotic solvent systems compatible with isobutane.

We then prepared and tested the complexes analogous to $\mathrm{V}$ and $\mathrm{VI}$ in which $n-\mathrm{C}_{3} \mathrm{~F}_{7}$ groups were substituted for $\mathrm{CF}_{3}$ (complexes VII and VIII respectively). Both of these new complexes were readily soluble in benzene, and isobutane oxidations could be smoothly run in this solvent, Table 1. Unexpectedly, an induction period of four to five hours was observed in these reactions. After this 
time, however, smooth and rapid oxidation of isobutane occurred giving tert-butyl alcohol as the major product, eq.1. The complex with four pendant $n-C_{3} F_{7}$ groups, bis $[5,10,15,20$-tetrakis-

(heptafluoropropyl)porphyrinatoiron(III)] $\mu$-oxide, VIII, was not only very active after the initial induction period, but reaction rates were then comparable with those of the tetrakis(pentafluorophenyl)porphyrin complex, III. This is the first demonstration of catalytic oxidation activity for metalloporphyrin complexes with perhaloalkyl groups on the periphery of the porphryin ring and further extends the general observation that although metalloporphyrin complexes are generally poor alkane oxidation catalysts, the presence of electron withdrawing groups on the periphery of the porphyrin ring, results in high catalytic activity.

Electron-deficient metalloporphyrin complexes that are active catalysts for the selective low temperature oxidations of light alkanes are also highly efficient catalysts for the selective decomposition of alkylhydroperoxides to alcohols (4), eq.2. Iron complexes having perhaloalkyl groups in the meso- positions are no exception to this trend. Table 1 indicates that when some or all of the meso-perfluorophenyl groups in an iron porphyrin complex are replaced with perfluoroalkyl groups, similarly high hydroperoxide decomposition activity is observed.

$$
\left(\mathrm{CH}_{3}\right)_{3} \mathrm{COOH} \longrightarrow\left(\mathrm{CH}_{3}\right)_{3} \mathrm{COH}+1 / 2 \mathrm{O}_{2}
$$

We have shown in earlier reports that halogenation of the pyrrolic positions of an electron deficient metalloporphyrin complex causes an increase in the catalytic activity of the metal complex. Table 2 shows that the same holds true for a complex having meso-trifluoromethyl groups. As the number of pyrrolic hydrogens replaced by bromo substituents increases, the catalytic activity of the complex steadily increases as well. Thus, by adding a perfluoroalkyl group in the meso- position we create an active catalyst, and then by brominating the pyrrolic position one can enhance catalytic activity even further.

\subsection{CONCLUSIONS}

The unprecedented activity for selective light alkane oxidation observed when electron-deficient metalloporphyrins are used as catalysts encourages one to consider their potential practical application. A major impediment to the use of perhaloporphyrinato metal porphryins is their high cost. The perfluoroalkyl moiety can be introduced into a dipyrromethane via a relatively inexpensive perfluoroaldehyde in up to $70 \%$ yield. The overall cyclization step (7) of the resulting dipyrromethane with an aldehyde is quite efficient and also occurs in good yield. Catalysts which are both highly active and inexpensive are very desirable for the direct air-oxidation of isobutane to tert-butyl alcohol.

This work not only provides a novel method for creating a wide range of new and potentially less expensive porphyrinatometal complexes, but further expands the range of electron withdrawing substituents which can be used to enhance activity. Work continues toward practical catalysts for light alkane oxidation based on first row transition metals in highly electron deficient porphyrinic macrocycles. 


\subsection{REFERENCES}

1. J. E. Lyons and P.E. Ellis, Jr., "Metalloporphyrins in Catalytic Oxidations," R. A. Sheldon, ed., Marcel Dekker, New York, 291-324 (1994) and references cited therein.

2. J. E. Bartoli, P. Battioni, W. R. Defoor and D. Mansuy, L. Chem Soc. Chem. Commun., 23 (1994).

3. J. E. Lyons, P. E. Ellis, Jr., H. K. Myers, Jr. and R. W. Wagner, I. Catal., 141, 311 (1993).

4. P. E. Ellis, Jr., J. E. Lyons and S. N. Shaikh, Catal Lett., 24, 79 (1993).

5. M. W. Grinstaff, M. G. H. Hill, J. Labinger and H. B. Gray; Science, 264, 1311 (1994).

6. J. E. Lyons and P. E. Ellis, Jr., Catal Lett., $\underline{8}, 45$ (1991).

7. T. P. Wijesekera, J. Org. Chem, submitted.

8. Other synthetic approaches to these compounds include:

a) T. P. Wijesekera and R. W. Wagner, U.S.Patent 5,241,062 (1994)

b) S. G. DiMagno, R. A. Williams and M. J. Therien, L. Org. Chem., 59, 6943 (1994).

9. K. Jayaraj, A. Gold, G. E. Toney, J. H. Helms, W. D. Hatfield, Inorg. Chem., 25, 3516 (1986).

10. J. E. Lyons, P. E. Ellis, Jr., H. K. Myers, Jr., J. Catal, (in press). 
TABLE I Effect of meso-perfuoroalkyl Substituents on the catalytic activity of electron deficient iron porphyrin complexes

\begin{tabular}{|c|c|c|c|c|c|c|c|c|c|}
\hline & \multirow[b]{2}{*}{ Catalyst } & \multirow[b]{2}{*}{ meso-Groups } & \multirow[b]{2}{*}{ B-Groups } & \multirow{2}{*}{$\begin{array}{c}\text { Axial } \\
\text { Ligand.LE }\end{array}$} & \multicolumn{2}{|c|}{ Isobutane Oxidations } & \multirow[b]{2}{*}{ TBA Sel $\%$} & \multicolumn{2}{|c|}{ TBHP Decompositions ${ }^{b}$} \\
\hline & & & & & Time, $h r^{d}$ & TO & & Time $\mathrm{Hr}^{d}$ & $\mathrm{TQ}^{*}$ \\
\hline & 1. & $4 \mathrm{C}_{6} \mathrm{H}_{5}$ & $8 \mathrm{H}$ & $\mathrm{OH}$ & 12 & 0 & - & 1 & 13,040 \\
\hline & 11 & $4 \mathrm{C}_{6} \mathrm{H}_{5}$ & $8 \mathrm{H}$ & $\mu-O$ & 12 & 0 & - & & \\
\hline & III. & $4 \mathrm{C}_{6} \mathrm{~F}_{5}$ & $8 \mathrm{H}$ & $\mathrm{OH}$ & 6 & 1090 & 90 & 1 & 66,200 \\
\hline & V. & $2 \mathrm{C}_{6} \mathrm{~F}_{5}, 2 \mathrm{CF}_{3}$ & $8 \mathrm{H}$ & $\mu-O$ & 6 & 530 & 90 & 1 & 62,500 \\
\hline & VII. & $2 \mathrm{C}_{6} \mathrm{~F}_{5}, 2 \mathrm{C}_{3} \mathrm{~F}_{7}$ & $8 \mathrm{H}$ & $\mu-O$ & $6^{h}$ & 90 & NA & 1 & 72,160 \\
\hline & & & & & 12 & 710 & 90 & & . \\
\hline & VIII & $4 \mathrm{C}_{3} \mathrm{~F}_{7}$ & $8 \mathrm{H}$ & $\mu-O$ & $6^{\mathrm{h}}$ & 110 & NA & 1 & 82,780 \\
\hline & & & & & 12 & 1110 & 81 & & \\
\hline
\end{tabular}

a)Approximately 7 grams isobutane was bubbled into a chilled solution of $\sim 0.013$ mmoles of catalyst in $25 \mathrm{ml}$ benzene in a Fisher-Porter aerosol tube. The tube was fitted to a gas manifold, flushed with $\mathrm{O}_{2}$ and then filled with pure $\mathrm{O}_{2}$. The tube was plunged into a constant temperature bath at $60^{\circ} \mathrm{C} \pm 2^{\circ} \mathrm{C}$ and filled with $\mathrm{O}_{2}$ to $100 \mathrm{psig}$. The solution was stirred magnetically at $60^{\circ} \mathrm{C}$ for 6 hours between 90 and 100 psig total gas pressure. As $\mathrm{O}_{2}$ was consumed, it was replenished from a reservoir. After 6 hours reaction mixture was cooled, returned to atmospheric pressure and the liquid analyzed by standard glpc. b) The catalyst, $0.60 \mathrm{mg}$, was added directly to a stirred solution of tert-butyl hydroperoxide, $13.8 \mathrm{~g}$, in tert-butyl alcohol, $18.1 \mathrm{~g}$, at $80^{\circ} \mathrm{C}$. Oxygen evolution was measured manometrically. Liquid products were analyzed by glpc before and after the runs. c) Either the axial ligand was $\mathrm{OH}$ or the complex was a $\mu$-oxo complex;(Porph)FeOFe(Porph). d)Reaction time. e)moles oxygen absorbed/equivalent of iron used. f)moles tert-butyl alcohol produced)/(moles liquid product)X100. g)moles oxygen evolved/equivalent of iron used. $h$ )Prolonged induction periods were observed after which facile reaction occurred. 
TABLE 2 Effect of $\beta$-Bromination of $\mathrm{III}$ and V on Catalytic Isobutane Oxidation Activity"

\begin{tabular}{|c|c|c|c|c|c|c|}
\hline Catalyst & meso-Groups & B-Groups ${ }^{b}$ & $\begin{array}{l}\text { Axial } \\
\text { Ligand }^{\circ}\end{array}$ & $\begin{array}{l}\text { Reaction } \\
\text { Time hrs }\end{array}$ & $\begin{array}{l}\mathrm{O}_{2} \\
\mathrm{TO}^{\mathrm{d}}\end{array}$ & IBA.Sel.\%" \\
\hline III & $4 \mathrm{C}_{6} \mathrm{~F}_{5}$ & $8 \mathrm{H}$ & $\mathrm{OH}$ & 6 & 1090 & 88 \\
\hline IIIA & $4 C_{6} F_{5}$ & $4 \mathrm{Br}, 4 \mathrm{H}$ & $\mathrm{OH}$ & 6 & 1220 & 86 \\
\hline IV & $4 C_{6} F_{5}$ & $8 \mathrm{Br}$ & $\mathrm{OH}$ & 6 & 1620 & 86 \\
\hline V & $2 \mathrm{C}_{6} \mathrm{~F}_{5} 2 \mathrm{CF}_{3}$ & $8 \mathrm{H}$ & $\mu-O$ & 6 & 530 & 90 \\
\hline $\mathrm{Va}$ & $2 \mathrm{C}_{6} \mathrm{~F}_{5} 2 \mathrm{CF}_{3}$ & $2 \mathrm{Br}, 6 \mathrm{H}$ & $\mu-O$ & 6 & 600 & 88 \\
\hline $\mathrm{Vb}$ & $2 \mathrm{C}_{6} \mathrm{~F}_{9} 2 \mathrm{CF}_{3}$ & $4 \mathrm{Br}, 4 \mathrm{H}$ & $\mu-\mathrm{O}, \mathrm{OH}$ & 6 & 960 & 89 \\
\hline$V_{c}$ & $2 \mathrm{C}_{6} \mathrm{~F}_{5} 2 \mathrm{CF}_{3}$ & $5 \mathrm{Br}, 3 \mathrm{H}$ & $\mu-\mathrm{O}, \mathrm{OH}$ & 6 & 1045 & 88 \\
\hline $\mathrm{Vd}$ & $2 \mathrm{C}_{6} \mathrm{~F}_{5} 2 \mathrm{CF}_{3}$ & $7 \mathrm{Br}, 1 \mathrm{H}$ & $\mathrm{OH}$ & 6 & 1330 & 88 \\
\hline
\end{tabular}

a)Approximately 7 grams isobutane was bubbled into a chilled solution of $\sim 0.013$ mmoles of catalyst in $25 \mathrm{ml}$ benzene in a Fisher-Porter Aerosol tube. The tube was fitted to a gas manifold, flushed with $\mathrm{O}_{3}$ and then filled with pure $\mathrm{O}_{2}$. The tube was plunged into a constant temperature bath at $60^{\circ} \mathrm{C} \pm 2^{\circ} \mathrm{C}$ and filled with $\mathrm{O}_{2}$ to $100 \mathrm{psig}$. The solution was stirred magnetically at $60^{\circ} \mathrm{C}$ for 6 hours between 90 and 100 psig total gas pressure. As $\mathrm{O}_{2}$ was consumed, it was replenished from a reservoir. After 6 hours the reaction mixture was cooled, returned to atmospheric pressure and the liquid analyzed by standardized gipc. b) Bromination of compound $V$ under different conditions led to product mixtures $V$ having different amounts of substitution of bromine into the $\beta$-position. The numbers of $\beta$-groups given in this Table refer to the most abundant compound in the mixture. c)With low $\mathrm{Br}$ substitution the product was recovered as the $\mu$-oxo dimer; with high $\mathrm{Br}$ substitution as the hydroxo complex; with intermediate $\mathrm{Br}$ substitution the product was a mixture of hydroxo and and $\mu$-oxo complexes. 
Figure 1 The substituted porphyrinato ring system: meso-substituents $=\mathrm{C}_{6} \mathrm{H}_{3}, \mathrm{C}_{6} \mathrm{~F}_{5}, \mathrm{CF}_{3}, \mathrm{C}_{3} \mathrm{~F}_{7}$, $\beta$-groups $=\mathrm{H}, \mathrm{Br}$; axial ligand=OH, $\mu$-Oxo (PFeOFeP)

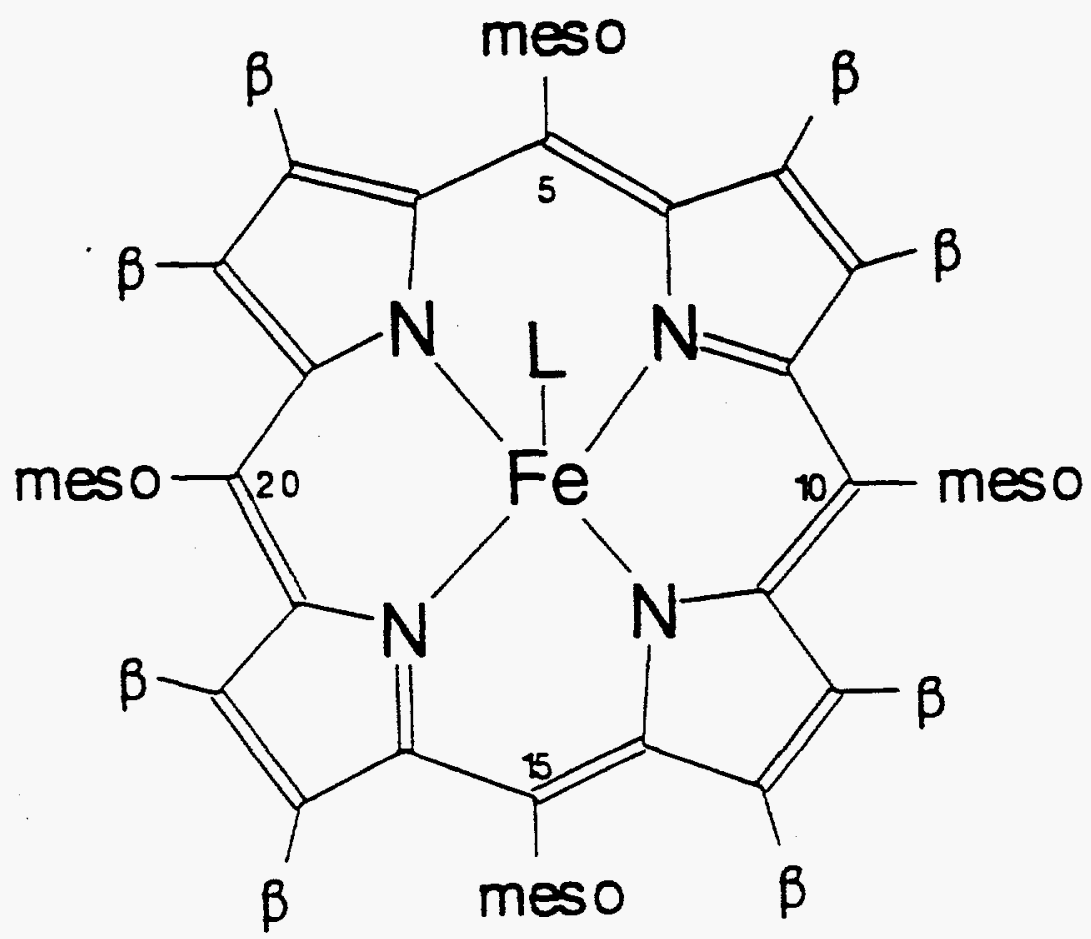




\section{0 meso-PERELUOROALKYL DIPYRROMETHANES AND PORPHORNS THEREFROM}

A two-step procedure is described for the synthesis of meso-perfluoroalkylporphyrins starting from pyrrole and perfluoroalkyl aldehydes via the intermediacy of 5-perfluoroalkyldipyrromethanes.

Electron-deficiency created at the metal center has been shown to increase the catalytic activity of porphyrinatoiron (III) complexes towards hydroxylation of alkanes by oxygen and other oxidants ${ }^{1}$. The electron-withdrawing groups most commonly employed at the meso-positions are the perhalophenyls, because of the easy accessibility of meso-tetraphenylporphyrins by the direct condensation of pyrrole and the corresponding aldehyde ${ }^{2}$, and the ease of functionalization of the beta-positions of a preformed meso-tetraphenylporphyrin ${ }^{3}$. It has been shown ${ }^{4}$ that meso-tetrakis(pentafluorophenyl)porphyrinatoiron(III) catalyzes the oxidation of isobutane to tert-butyl alcohol ( $>90 \%$ selectivity) in the presence of air or oxygen as the sole oxidant with $>3700$ $\mathrm{TO} / \mathrm{h}$ at $80^{\circ} \mathrm{C}$, while beta perhalogenation of the catalyst leads to $>5700 \mathrm{TO} / \mathrm{h}$ at the same temperature ${ }^{3 \mathrm{c}, 4}$. However, due to the practical implications of the high cost (and the high mass per iron center) of catalysts carrying perfluorophenyl groups, our attention was directed towards the synthesis of metalloporphyrin systems having perfluoroalkyl groups at the meso-positions. This was particularly important since a recent study of substituent effects on porphyrin systems using X-ray photoelectron spectroscopy and ab initio calculations suggested that a trifluoromethyl group at the meso-position could create significant electron-deficiency at the core nitrogen atoms (and hence the metal center) of a porphyrin system ${ }^{5}$. We report here a general synthetic route to porphyrins carrying perfluoroalkyl groups at opposite meso-positions $(5,15-)$ while the substituents at the other two meso-positions $(10,20-)$ can be varied as desired. The key intermediate in this synthesis is the 5-perfluoroalkyldipyrromethane.

Carbon bonded alkyl and aryl substituents are generally incorporated into the porphyrin ring either before or during macrocyclization ${ }^{2}$. Although acid catalyzed co-condensation of pyrrole and an aldehyde produces meso-aryl or meso-alkyl porphyrins ${ }^{6.7}$ in good yields, attempts to prepare meso-tetrakis(trifluoromethyl)porphyrin, even under harsher reaction conditions, have produced very low quantities of porphyrin. This is due to the powerful destabilizing effect the $\mathrm{CF}_{3}$ group has on the formation of a carbenium ion adjacent to it, which has been shown to result in $k(H) / k\left(\mathrm{CF}_{3}\right)$ ratios of $10^{6}$ or more ${ }^{9}$. However, the reaction of pyrrole 1 and trifluoroacetaldehyde $2 \mathrm{a}$ (as the methyl or ethyl hemiacetal) in refluxing $\mathrm{THF}$ and $\mathrm{c.HCl}(40: 1)$ produced the 5-trifluoromethyldipyrromethane $3 \mathrm{a}^{\mathbf{1 0}}$ (Scheme 1) in yields up to $70 \%$ when a $2: 1$ molar ratio of pyrrole to aldehyde was used. Reaction times greater than 2-3h and/or stronger acid conditions generally led to increased quantities of oxidized and/or polymerized by-products, although no porphyrin could be detected. After neutralization of the acid catalyst and removal of the solvent the product was extracted from the orange-red oil into petroleum ether $\left(30-60^{\circ} \mathrm{C}\right)$ and purified on neutral alumina to give the dipyrromethane in $>98 \%$ purity. Substitution of trifluoroacetaldehyde 2a with heptafluorobutyraldehyde $\mathbf{2 b}$ (used as the hydrate) in the above condensation reaction gave the corresponding 5-heptafluoropropyldipyrromethane $\mathbf{3 b}^{11}$ in comparable yields. The dipyrromethanes are sensitive to the acid concentration in solution and the work up procedure was found to affect the isolated yields significantly although the purified solid is stable for several months in the refrigerator. Recently, Vigmond et.al. ${ }^{12}$ prepared a series of $\beta$-unsubstituted-5-aryldipyrromethanes by the direct 
condensation of pyrrole and the corresponding benzaldehyde in THF and acetic acid (9:1) at low temperatures. Mizutani et. al. ${ }^{13}$ and Lee et. al. ${ }^{14}$ reported similar condensations under different acid conditions using a large molar excess of pyrrole ( $>40: 1)$, conditions not suitable for a practical process.

5-Perfluoroalkyldipyrromethanes $3 \mathrm{a}$ and $3 \mathrm{~b}$ were co-condensed with trifluoroacetaldehyde, heptafluorobutyraldehyde and pentafluorobenzaldehyde (Scheme 2), in $\mathrm{CHCl}_{3}$ under acid conditions to give, after oxidation with $\mathrm{DDQ}$, the electron-deficient porphyrins $5 \mathrm{a}-\mathrm{d}^{3 \mathrm{~s}}$. Of the several acid catalysts that have previously been employed for this purpose ${ }^{16}$, Montmorillonite K10 clay ${ }^{17}$ not only gave the best isolated yields of the porphyrins, but also facilitated the purification procedure since most of the polypyrrolic impurities were adsorbed on the clay. The porphyrin was isolated following oxidation of the reaction mixture with DDQ and filtering through a bed of neutral alumina. Condensation of the dipyrromethanes with perfluoroalkyl aldehydes $\mathbf{4 b}$ and $4 \mathrm{c}$ required longer reaction times (15-20h) at reflux temperature, and the yields of porphyrins were lower (5-10\%), compared with the condensations with pentafluorobenzaldehyde $4 \mathrm{a}$, which were carried out at room temperature requiring shorter reaction times $(8-10 \mathrm{~h})$, and producing higher yields $(15-20 \%)$ of the product. The reaction conditions are currently being optimized.

A recent publication ${ }^{18}$ described the synthesis of meso-tetrakis(heptafluoropropyl)porphyrin $\mathbf{5 d}$ by the tetramerization-cyclization of the heptafluoropropyl substituted 2-(hydroxymethyl)pyrrole, under rigorous elimination of water. We had previously reported the use of the trifluoromethyl analogoue of such 2-(hydroxymethyl)pyrroles for the synthesis of meso-tetrakis(trifluoromethyl)porphyrin $\mathbf{5 b}$ by the prior activation of the hydroxyl group with a superior leaving group ${ }^{19}$. However, the two-step procedure described here provides a general method for the synthesis of porphyrins that have perfluoroalkyl groups at opposite meso-positions while the other two meso positions may have the same or different perhalocarbyl groups. This allows us to compare the effect of perhaloalkyl groups with that of other electron-withdrawing groups on the catalytic activity of porphyrinatoiron(III) systems. Iron insertion and $\beta$-halogenation of the meso-perfluorocarbyl-

porphyrins 5a-d have been carried out using modifications of literature procedures ${ }^{2}$ and the catalytic studies will be reported elsewhere ${ }^{20}$.

\section{Acknowledgments}

This work was supported by the U.S. Department of Energy (Morgantown Energy Technology Center), the Gas Research Institute and Sun Company. The author wishes to thank Dr. R.A. Merrill and Dr. K.S. Norbash for providing the spectroscopic information of the new compounds.

\subsection{REFERENCES AND NOTES}

1. (a) Lyons, J.E.; Ellis, P.E., Jr. In Metalloporphyrins in Catalytic Oxidations; Sheldon, R. Ed.; Marcel Dekker: N.Y., 1994, pp 297-324 (b) Mansuy, D.; Battioni, P. In Metalloporphyrins in Catalytic Oxidations; Sheldon, R. Ed.; Marcel Dekker: N.Y., 1994, pp 99-132. (c) Meunier, B. Chem. Rev., 1992, 92, 1411-1456. 


\subsection{REFERENCES AND NOTES}

(CONTINUED)

2. Wijesekera, T.P.; Dolphin, D, In Metalloporphyrins in Catalytic Oxidations; Sheldon, R. Ed.; Marcel Dekker: N.Y., 1994, pp 193-239.

3. (a). Traylor, T.G.; Tsuchia, S. Inorg. Chem., 1987, 26, 1338-1339. (b) Wijesekera, T.P.; Matsumoto, A.; Dolphin, D.; Lexa, D. Angew. Chem. Int. Ed. Engl., 1990, 29, 1028-1030 (c) Lyons, J.E.; Ellis, P.E. Jr., Catalysis Lett. 1991, 8, 45-52. (d). Rocha Gonsalves, A.M. d'A; Johnstone, R.A.W.; Pereira, M.M.; Shaw, J.; Sobral, A.J.F. do N. Tetrahedron Lett., 1991, 32, 1355-1358. (e) Bartoli, J.F.; Brigaud, O.; Battioni, P.; Mansuy, D. J. Chem. Soc. Chem. Commun., 1991, 440-442. (f). Hoffmann, P.; Robert, A.; Meunier, B. Bull. Chem. Soc. Fr., 1992, 129, 85-97. (g) Bartoli, J.F.; Battioni, P.; De Foor, W.R.; Mansuy, D. J. Chem. Soc. Chem. Commun., 1994, 23-24.

4. Ellis, P.E. Jr.; Lyons, J.E., Coord. Chem. Rev., 1990, 105, 181-193.

5. Gassman, P.G.; Ghosh A.; Almlof, J. J. Am. Chem. Soc., 1992, 114, 9990-10000.

6. Adler, A.D.; Longo, F.R.; Finarelli, J.; Goldmacher, J.; Assour, J.; Korsakoff, L. J. Org. Chem., 1967, 32, 476.

7. Lindsey, J.S.; Schreiman, I.C.; Hsu, H.C.; Kearney, P.C.; Marguerettaz, A.M. J. Org. Chem., 1987, 52, 827-836

8. Ellis, P.E., Jr.; Lyons, J.E. U.S. Patent No. 4,970,348, 1990

9. Allen, A.D.; Kwong-Chip, J-M.; Lin, W.C.; Nguyen, P.; Tidwell, T.T. Can. J. Chem., $1990,68,1709-1713$, and references cited therein.

10. Analytical data for 3a: $\quad \mathrm{mp} 54-56^{\circ} \mathrm{C} ;{ }^{1} \mathrm{H} \mathrm{NMR}\left(\mathrm{CDCl}_{3}, 300 \mathrm{MHz}\right) \delta(\mathrm{ppm}): 7.96(\mathrm{bs}$, $2 \mathrm{H}), 6.73(\mathrm{~s}, 2 \mathrm{H}), 6.24(\mathrm{~m}, 4 \mathrm{H}), 4.80(\mathrm{q}, 1 \mathrm{H}) ;{ }^{19} \mathrm{~F} \mathrm{NMR}\left(\mathrm{CDCl}_{3}\right) \delta(\mathrm{ppm}): 68.05(\mathrm{~d})$; accurate mass calcd for $\mathrm{C}_{10} \mathrm{H}_{9} \mathrm{~N}_{2} \mathrm{~F}_{3} 214.0718$, found by HRMS (FAB) $214.0712(\triangle \mathrm{M}=$ $-2.7 \mathrm{ppm}$ )

11. Analytical data for 3b: $\mathrm{mp} 52-54^{\circ} \mathrm{C} ;{ }^{1} \mathrm{H} \mathrm{NMR}\left(\mathrm{CDCl}_{3}\right), \delta 8.12(\mathrm{bs}, 2 \mathrm{H}), 6.75(\mathrm{~s}, 2 \mathrm{H})$, $6.23(\mathrm{~s}, 2 \mathrm{H}), 6.19(\mathrm{q}, 2 \mathrm{H}), 4.92(\mathrm{t}, 1 \mathrm{H}) ;{ }^{19} \mathrm{~F} \mathrm{NMR}\left(\mathrm{CDCl}_{3}\right) \delta-82.86(\mathrm{~m}, 3 \mathrm{~F}),-115.21(\mathrm{~m}$, $2 \mathrm{~F}),-127.11(\mathrm{t}, 2 \mathrm{~F})$; accurate mass calcd for $\mathrm{C}_{12} \mathrm{H}_{9} \mathrm{~N}_{2} \mathrm{~F}_{7} 314.0654$, found by HRMS (FAB) $314.0651(\Delta \mathrm{M}=-0.9 \mathrm{ppm})$,

12. Vigmond, S.J.; Chang, M.C.; Kallury, K.M.R.; Thompson, M. Tetrahedron Lett., 1994,35,2455-2458. 


\subsection{REFERENCES AND NOTES (CONTINUED)}

13. (a). Mizutani, T.; Ema, T.; Tomita, T.; Kuroda, Y.; Ogoshi, H. J. Chem. Soc., Chem. Commun., 1993, 520-522. (b). Mizutani, T.; Ema, T.; Tomita, T.; Kuroda, Y.; Ogoshi, H. J. Am. Chem. Soc., 1994, 116, 4240-4250.

14. Lee, C-H; Lindsey, J.S. Tetrahedron, 1994, 50, 11427-11440.

15. Analytical data for 5a: ' $\mathrm{H} \mathrm{NMR}\left(\mathrm{CDCl}_{3}, 300 \mathrm{MHz}\right) \delta(\mathrm{ppm}): 9.71(\mathrm{~s}, 4 \mathrm{H}), 8.91(\mathrm{~d}, 4 \mathrm{H})$, -2.73 (bs, $2 \mathrm{H}$ ). ${ }^{19} \mathrm{~F} \mathrm{NMR}\left(\mathrm{CDCl}_{3}\right) \delta$ (ppm): accurate mass calcd for $\mathrm{C}_{34} \mathrm{H}_{10} \mathrm{~N}_{4} \mathrm{~F}_{16} 778.0650$, found by HRMS (FAB) $778.0630(\Delta \mathrm{M}=-2.6 \mathrm{ppm})$. UV-vis, $\lambda_{\max }$ (rel.int.): 408 (14.91), 506 (2.13), 538 (1.45), 584 (1), 640 (1.16). 5b: ${ }^{1} \mathrm{H}$ NMR (CDCl, $\left.300 \mathrm{MHz}\right) \delta(\mathrm{ppm})$ : $9.61(\mathrm{~s}, 8 \mathrm{H}),-2.09(\mathrm{bs}, 2 \mathrm{H}) .{ }^{19} \mathrm{~F} \mathrm{NMR}\left(\mathrm{CDCl}_{3}\right) \delta(\mathrm{ppm})$ : . accurate mass calcd for $\mathrm{C}_{24} \mathrm{H}_{10} \mathrm{~N}_{4} \mathrm{~F}_{12}$ 582.0714, found by HRMS (FAB) 582.0735 ( $\triangle \mathrm{M}=3.6 \mathrm{ppm}$ ). UV-vis, $\lambda_{\max }$ (rel.int.): 404 (13.07), 508 (1.92), 544 (1.89), 594 (1), 648 (1.86). 5c: ${ }^{1} \mathrm{H}$ NMR $\left(\mathrm{CDCl}_{3}, 300 \mathrm{MHz}\right) \delta(\mathrm{ppm}): 9.58(\mathrm{~s}, 4 \mathrm{H}), 8.92(\mathrm{~d}, 4 \mathrm{H}),-2.58(\mathrm{bs}, 2 \mathrm{H}) ;{ }^{19} \mathrm{~F} \mathrm{NMR}\left(\mathrm{CDCl}_{3}\right)$ $\delta$ (ppm): $-81.22(\mathrm{br}, \mathrm{t} 6 \mathrm{~F}),-84.27(\mathrm{~m}, 4 \mathrm{~F}),-121.97(\mathrm{br}, \mathrm{t}, 4 \mathrm{~F}),-138.73(\mathrm{t}, 4 \mathrm{~F}),-153.10(\mathrm{t}$, $2 \mathrm{~F}),-163.37(\mathrm{t}, 4 \mathrm{~F})$; accurate mass calcd for $\mathrm{C}_{38} \mathrm{H}_{10} \mathrm{~N}_{4} \mathrm{~F}_{24} 978.0522$, found by HRMS (FAB) $978.0535\left(\triangle \mathrm{M}=1.3 \mathrm{ppm}\right.$ ) UV-vis, $\lambda_{\max }$ (rel.int.): 406 (14.13), 506 (2.06), 540 (1.58), $584(1), 638(1.42)$. 5d: ${ }^{1} \mathrm{H} \mathrm{NMR}\left(\mathrm{CDCl}_{3}, 300 \mathrm{MHz}\right) \delta(\mathrm{ppm}): 9.50(\mathrm{~s}, 8 \mathrm{H}),-2.30$ (bs, $2 \mathrm{H}) ;{ }^{19} \mathrm{~F} \mathrm{NMR}\left(\mathrm{CDCl}_{3}\right) \delta$. accurate mass calcd for $\mathrm{C}_{32} \mathrm{H}_{11} \mathrm{~N}_{4} \mathrm{~F}_{28}(\mathrm{M}+\mathrm{H}) 983.0537$ found by HRMS (FAB) $983.0548(\Delta \mathrm{M}=1.2 \mathrm{ppm})$. UV-vis, $\lambda_{\max }$ (rel. int.): 404 (11.70), 510 (1.74), 544 (1.49), $592(1), 646(1.51)$

16. (a). Ogoshi, H.; Sugimoto, H.; Nishiguchi, T.; Watanabe, T.; Matsuda, Y.; Yoshida, Z. Chemistry Lett., 1978, 29-32. (b). Gunter, M.J.; Mander, L.N. J. Org. Chem., 1981, 46, 4792-4795. (c) Manka, J.S.; Lawrence, D.S. Tetrahedron Lett., 1989, 30, 6989-6992. (d). Osuka, A.; Nagata, T.; Kobayashi F.; Maruyama, K. J. Heterocyclic Chem., 1990, 27, 1657-1659.

17. (a). Onaka, M.; Shinoda, T.; Izumi, Y.; Nolen, E. Chemistry Lett., 1993, 117-120. (b). Onaka, M.; Shinoda, T.; Izumi, Y.; Nolen, E. Tetrahedron Lett., 1993, 34, 2625-2628.

18. DiMagno, S.G.; Willams, R.A.; Therien, M.J. J. Org. Chem. 1994, 59, 6943-6948

19. Wijesekera, T.P.; Wagner, R.W. U.S. Patent No. 5,241,062, 1993

20. Wijesekera, T.P.; Lyons, J.E.; Ellis, P.E. Jr. Catalysis Lett., submitted. 

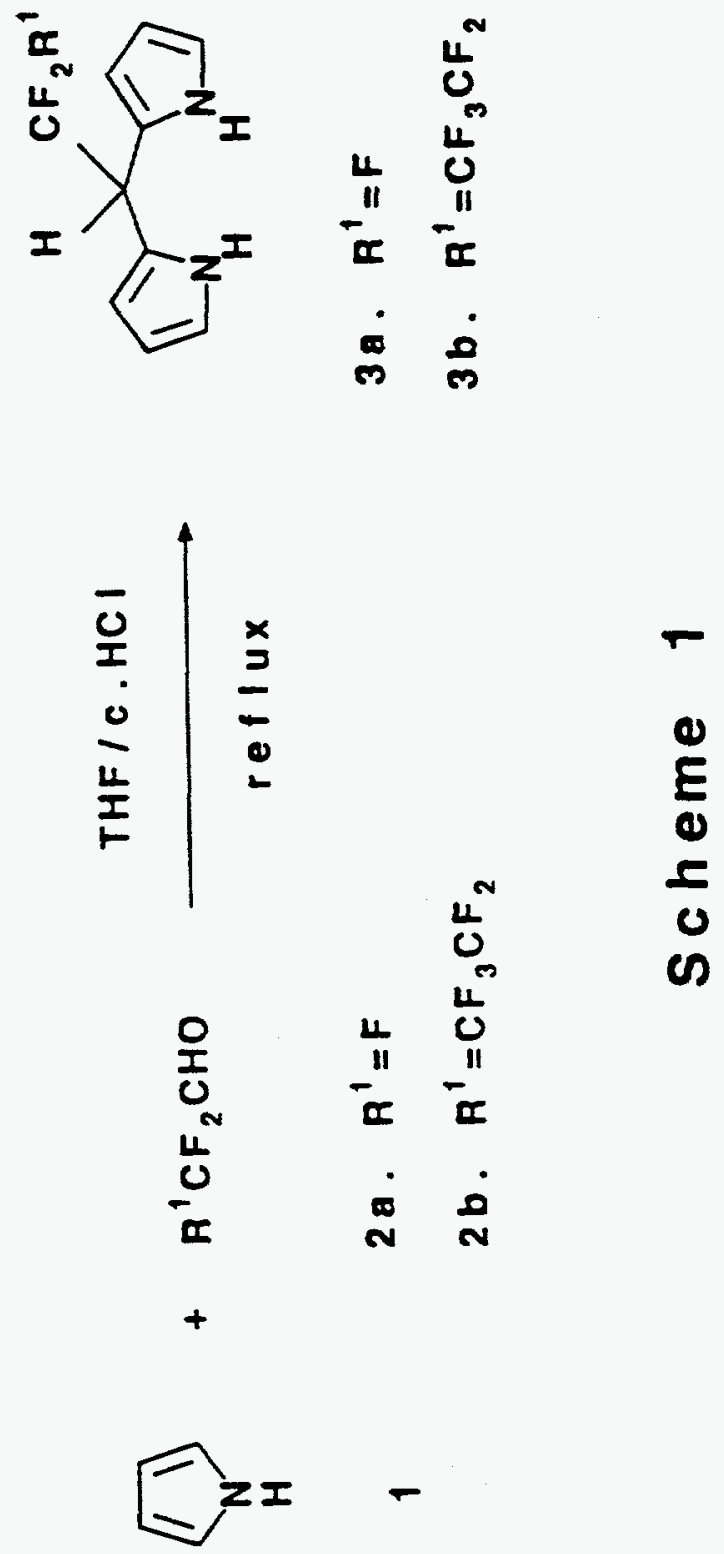

$\boldsymbol{N}$ 


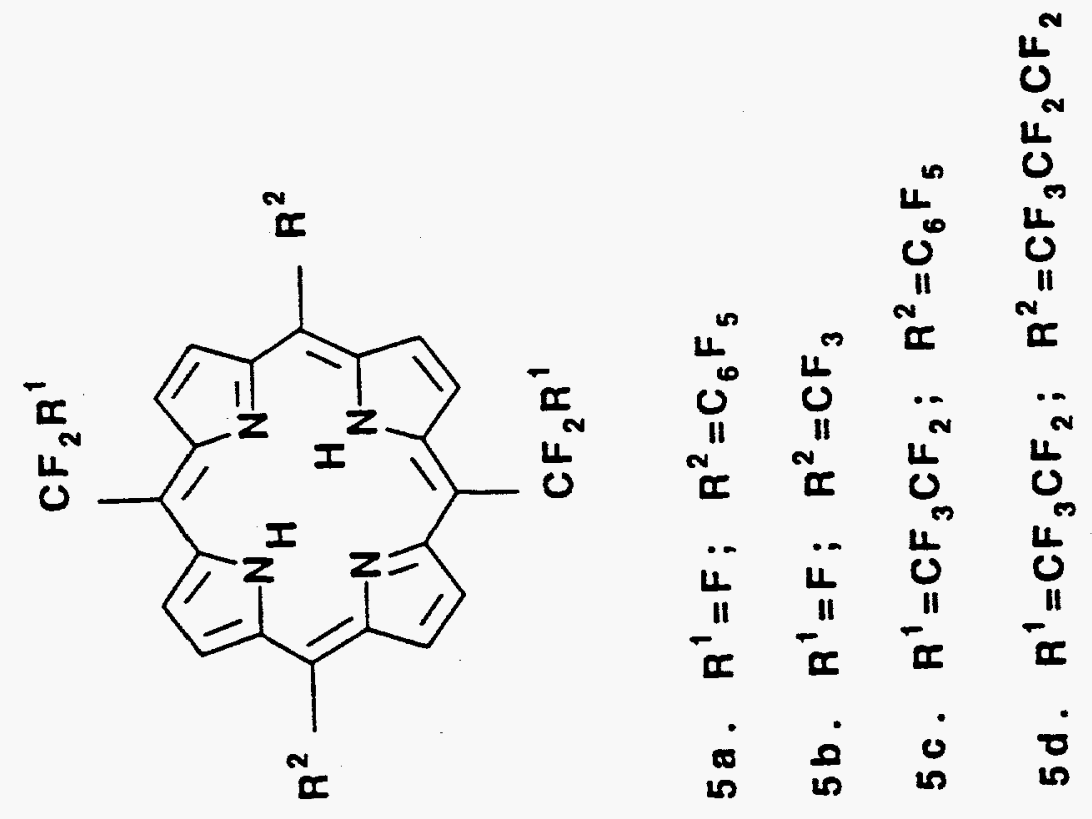

$\pm$

$\mathbf{N}$

$E$
$E$
0
0

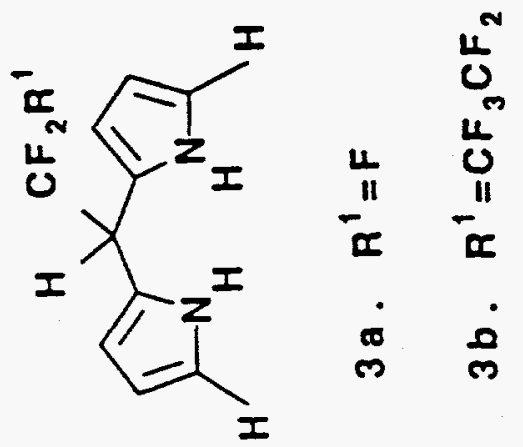




\subsection{NOVEL IRON mesO-NITROOCTAETHYLPORPHINE COMPLEXES FOR THE CATALYTIC REACTION OF ALKANES WITH MOLECULAR OXYGEN}

\subsection{INTRODUCTION}

The development of a catalyst for the selective oxidation of alkanes to alcohols with air or oxygen remains an important goal of both synthetic and industrial chemistry. Synthetic metalloporphyrin catalysts inspired by enzymic systems such as the monooxygenase cytochrome P450, are of interest as catalysts for alkane hydroxylations using a number of oxidants including molecular oxygen [1].

A key to the biomimetic approach to modeling cytochrome $\mathrm{P} 450$ and producing catalysts which can mimic the hydroxylation activity of the enzyme, has been the development of synthetic metalloporphyrin chemistry. Nearly all of the metalloporphyrins used as oxidation catalysts have been based on the meso-tetraphenylporphine, TPP, ligand system. A myriad of metalloporphyrin complexes have been synthesized in which both the meso-phenyl and pyrrolic groups have been substituted in order to adjust the electronic nature of TPP or modify the geometry of the porphyrin plane and thereby control access to the metal center [2].

In this regard, it is interesting to note that the prosthetic group of cytochrome P450, iron-protoporphyrin IX, contains no phenyl groups in the meso positions and in fact is unsubstituted in that positions. Synthetic "flat" metalloporphyrin complexes that have no meso-phenyl groups and are not protected by the protein as in the natural enzyme, have been ineffective for alkane oxidation [3]. It is thought that the meso-unsubstituted metalloporphyrin complexes are prone to attack at the meso-carbon during oxidation leading to facile porphyrin degradation $[4,5]$.

We have shown that the tetraphenylporphyrin metal complexes whose meso-phenyl groups bear halogen substitutents are active catalysts for the reaction of unsubstituted light alkanes with oxygen [2a,6-7]. In particular, complexes having meso-pentafluorophenyl substituents exhibit unprecedented activity for the air-oxidation of alkanes. We have further shown that this activity is related to the electron withdrawing character of the halogen substitutents and may be due to increased $M(\mathrm{III}) / \mathrm{M}$ (II) reduction potential at the central metal atom. To date, however, no meso-groups other than substituted phenyis have been shown to be effective for metlloporphyrin-catalyzed air oxidation of alkanes.

In this communication we report for the first time that incorporating highly electron-withdrawing nitro groups in the meso-position of a porphyrinato iron complex provides soluble, active catalysts for the liquid phase oxidation of alkanes. We further describe the synthesis of nitrated iron octaethylporphyrins and the effect of the degree of meso-nitration on catalytic activity. These are the first metalloporphyrin complexes having no phenyl substituents in the meso-position which are efficient catalysts for the oxidation of hydrocarbons with dioxygen. 


\subsection{EXPERIMENTAL}

The $\mu$-oxo dimer of (meso-tetranitrooctaethylporphinato)iron (III), $\left[\mathrm{Fe}(\mathrm{OEP})\left(\mathrm{NO}_{2}\right)_{4}\right]_{2} \mathrm{O}$, was prepared by nitration similar to the method of Gong and Dolphin [8] wherein a 12/1 molar ratio of $\mathrm{NO}_{2}$ to $\mathrm{Fe}(\mathrm{OEP}) \mathrm{Cl}$ is stirred under $\mathrm{N}_{2}$ in $\mathrm{CH}_{2} \mathrm{Cl}_{2}$ for 1 hour at room temperature. After chromatography on neutral alumina eluting with chloroform/hexane, the pure $\mu$-oxo dimer is obtained. FAB/MS gives a $\mathrm{M}^{+}$of 767.5 for the complex, corresponding to $\mathrm{Fe}(\mathrm{OEP})\left(\mathrm{NO}_{2}\right)_{4}^{+}$. The $\mathrm{IR}(\mathrm{KBr})$ spectrum shows $\mathrm{N}-\mathrm{O}$ stretches at 1368 and $1537 \mathrm{~cm}^{-1}$ and the $\mathrm{Fe}-\mathrm{O}-\mathrm{Fe}$ stretch at $853 \mathrm{~cm}^{-1}$. The ${ }^{1} \mathrm{H}$ NMR $\left(\mathrm{CDCl}_{3}\right)$ spectrum is very similar to that of the $\mu$-oxo dimer of Fe(OEP) with broad resonances at 5.8 and $4.5\left(-\mathrm{CH}_{2}-\right.$ and $1.36\left(-\mathrm{C} \mathscr{H}_{3}\right) \mathrm{ppm}$.

The chloride salt, $\mathrm{Fe}(\mathrm{OEP})\left(\mathrm{NO}_{2}\right)_{4} \mathrm{Cl}$ is obtained by treatment of the $\mu$-oxo dimer with $6 \mathrm{~N} \mathrm{HCl}$ in $\mathrm{CH}_{2} \mathrm{Cl}_{2}$. The ${ }^{1} \mathrm{H} \mathrm{NMR}\left(\mathrm{CDCl}_{3}\right)$ spectrum of this material shows resonances at $39.2\left(-\mathrm{CH}_{2}-\right)$ and 5.4 $\left(-\mathrm{C} \mathscr{H}_{3}\right) \mathrm{ppm}$.

$\left\{\mathrm{Fe}\left[5,15-\left(\mathrm{NO}_{2}\right)_{2} \mathrm{OEP}\right]\right\}_{2} \mathrm{O}[10]$, a by-product of nitration, is obtained via chromotography on neutral alumina eluting with a $1 / 3 \mathrm{CH}_{2} \mathrm{Cl}_{2}$ /hexane mixture. This material is recrystallized from slow addition of an isopropanol/hexane mixture to $\mathrm{CH}_{2} \mathrm{Cl}_{2}$ solution of the dinitro iron porphyrin. ' $\mathrm{H}$ NMR $\left(\mathrm{CDCl}_{3}\right)$ has broad resonances at 6.5 (meso $\left.\mathrm{C}-\mathcal{H}_{2}\right), 6.0,5.8,5.0,4.3\left(-\mathrm{CH}_{2}-\right)$ and 1.7 and $1.5\left(-\mathrm{CH}_{3}\right)$. The IR (KBr) spectrum shows N-O stretches at 1367 and $1533 \mathrm{~cm}^{-1}$ and the $\mathrm{Fe}-\mathrm{O}-\mathrm{Fe}$ stretch of the $\mu$-oxo dimer is seen as a shoulder at $860 \mathrm{~cm}^{-1}$. The IR also shows a strong band at $875 \mathrm{~cm}^{-1}$ attributable to a meso-CH deformation mode [11] which is not present in the tetranitrated material in which all four meso- $\mathrm{CH}$ bands have been nitrated. [Fe(OEP)] $]_{2} \mathrm{O}$ was prepared [9] by reaction of $\mathrm{Fe}(\mathrm{OEP}) \mathrm{Cl}$ with aqueous sodium hydroxide.

Isobutane oxidations were conducted in glass Fisher-Porter aerosol tubes attached to a gas manifold for addition of pure oxygen as it was consumed. Isobutane was dissolved in a benzene solution of the catalyst which was stirred magnetically and heated by immersion of the tube in an oil bath. Product analyses were conducted by standardized glpc.

Propane oxidations were carried out under air pressure in $300 \mathrm{cc}$ glass lined stainless steel autoclaves equipped with internal thermocouple, magnedrive stirrer and cooling coils for regulating reaction temperature. Internals were teflon-coated. Propane was pressured into a benzene solution of the catalyst and air was then added under pressure. Product analyses were conducted by standardized gipc.

Caution: Some reactions were conducted within the explosive region so care was taken to properly barricade reactor systems. Glass reactors for isobutane oxidations were housed inside hoods surrounded by one-inch polycarbonate shielding. Propane oxidations were carried out behind barricades of steel and sand. 


\subsection{RESULTS AND DISCUSSION}

Table 1 shows that the electron-withdrawing nitro group in the mesa-position of the porphyrin macrocycle, Figure 1, imparts hydrocarbon oxidation activity to the otherwise inactive iron octaethylporphyrin complex. The greater the number of nitro groups, the more active is the porphyrin complex for air-oxidation of isobutane. The Fe(III)/(II) reduction potentials of the octaethylporphyrin complexes increase steadily with each successive meso-nitro group [10]. In addition, the stability of the complex under oxidation conditions becomes greater as nitro groups are added to the meso-positions around the macrocycle.

As shown in Table 1, the diiron $\mu$-oxo complex is highly active for alkane oxidation. It is very soluble in hydrocarbon medium and is a convenient homogeneous catalyst for this reaction. High activity is characteristic of the bismetal $\mu$-oxo complexes of electron deficient porphyrins $[2 \mathrm{a}, \mathrm{b}]$ in contrast to typical metalloporphyrins whose $\mu$-oxo dimers are inactive. Selectivity of the isobutane oxidation reactions to the alcohol is high $(88 \%)$ in these catalytic reactions.

Because of the eight electron-donating ethyl groups in the beta-positions of the octaethylporphine complexes, one would not expect these catalysts to be as highly activeas a complex which has electron-withdrawing substituents in the beta-positions. We have also observed that these complexes do not have as long a life under oxidation conditions as do the perhaloporphyrins referred to in the introduction [2]. Again the beta-ethyl groups may be particularly susceptible to radical attack during oxidation.

The $\mu$-oxo dimer, $\left[\mathrm{Fe}(\mathrm{OEP})\left(\mathrm{NO}_{2}\right)_{4}\right]_{2} \mathrm{O}$, also catalyzes the reaction of propane with molecular oxygen. Propane, 1.36 moles, was oxidized using $1500 \mathrm{psi}$ air in a stirred glass-lined autoclave in 48 $\mathrm{ml}$ benzene containing 0.023 mmoles of $\left[\mathrm{Fe}(\mathrm{OEP})\left(\mathrm{NO}_{2}\right)_{4}\right]_{2} \mathrm{O}$ at $125^{\circ} \mathrm{C}$ for six hours. Acetone and isopropyl alcohol in a $2 / 1$ ratio were the only oxidation products detected in the liquid phase. The turnover number was 148 moles of liquid oxidation products per mole of catalyst in the six hour period. After this time the dark benzene solution of catalysts had nearly completely bleached and the catalyst had deactivated.

\subsection{CONCLUSIONS}

Incorporating nitro groups into the meso-position of octaethylporphinato iron complexes introduces a powerful electron withdrawing group which increases the $\mathrm{Fe}(\mathrm{III}) / \mathrm{Fe}(\mathrm{II})$ reduction potential over that of the parent $\mathrm{Fe}(\mathrm{OEP})$ complex and provides catalysts for the selective, low temperature oxidation of alkanes. Thus, the nitro groups serve both to protect the reactive mesa-position from rapid oxidative attack and to enhance the catalytic oxidation activity. In addition, these findings show the validity of the general hypothesis that electron withdrawing groups in the meso-position of porphinato iron complexes enhance alkane oxidation activity [2a]. Work continues to examine this phenomenon in more detail as well as to further enhance catalytic activity and stability for selective alkane oxidations. 


\subsection{REFERENCES}

1. B. Meunier, Chem. Rev. 92 (1992) 1411.

2a. P. E. Ellis, Jr. and J. E. Lyons, Coord. Chem. Rev. 105 (1990) 181.

b. J. E. Lyons and P. E. Ellis, Jr., Cat. Lett. 8 (1991) 45.

c. J. F. Bartoli, O. Brigaud, P. Battioni, and D. Mansuy, J. Chem. Soc. Chem. Comm. (1991) 440.

d. T. Wijesekera, A. Matsumoto, D. Dolphin, and D. Lexa, Angew. Chem. Int. Ed. Engl. 29 (1990) 1028.

3. J. H. Fuhrhop, M. Baccouche, H. Graboy, and H. Arzomanian, J. Mol. Cat. 7 (1980) 245.

4. C. K. Chang and M. S. Kuo, J. Amer. Chem. Soc. 101 (1979) 3413.

5. J. A. S. Cavaleiro, M. J. E. Hewlins, A. H. Jackson, and G. P. M. S. Neves, J. Chem. Soc.Chem. Comm. (1986) 142.

6. P. E. Ellis, Jr., and J. E. Lyons, J. Chem. Soc. Chem. Comm. (1989) 1315.

7. P. E. Ellis, Jr. and J. E. Lyons, Cat. Lett. 3 (1989) 389.

8. L. C. Gong and D. Dolphin, Can. J. Chem. 63 (1985) 401.

9. G. N. La Mar, G. R. Eaton, R. H. Holm, and F. A. Walker, J. Am. Chem. Soc. 95 (1973 63.

10. L. C. Gong and X. L. Yang, Acta Chimica Sinica 43 (1985) 302.

11. E. Watanabe, S. Nishimura, H. Ogoshi, and Z. Yoshida, Tetrahedron 31 (1975) 1385. 
TABLE 1

ISOBUTANE OXIDATION ACTIVITY OE IRON PORPHYRIN COMPLEXES

COMPLEX
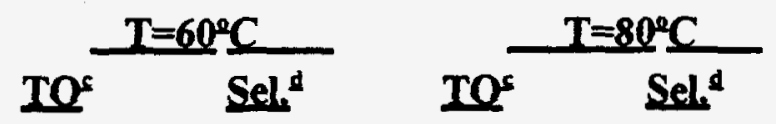

$[\mathrm{Fe}(\mathrm{OEP})]_{2} \mathrm{O}^{\mathrm{b}}$

0

0

$\left\{\mathrm{Fe}\left[5,15-\left(\mathrm{NO}_{2}\right)_{2} \mathrm{OEP}\right]\right\}_{2} \mathrm{O}$

360

88

970

89

$\left[\mathrm{Fe}(\mathrm{OEP})\left(\mathrm{NO}_{2}\right)_{4}\right]_{2} \mathrm{O}$

800

88

1680

88

Isobutane, $7 \mathrm{~g}$, in $30 \mathrm{ml}$ benzene containing 0.013 mmole complex, heated and stirred at reaction temperature for 5 hours under 100 psig of $\mathrm{O}_{2}$.

${ }^{b} \mathrm{OEP}=$ Octaethylporphyrinato

${ }^{\top} \mathrm{TO}=$ Turnovers $=$ moles $(\mathrm{TBA}+$ acetone $) /$ moles catalyst.

${ }^{\mathrm{d}}$ Sel. $=($ moles $\mathrm{t}$-butyl alcohol produced/total moles liquid product $\mathrm{x} 100$. 


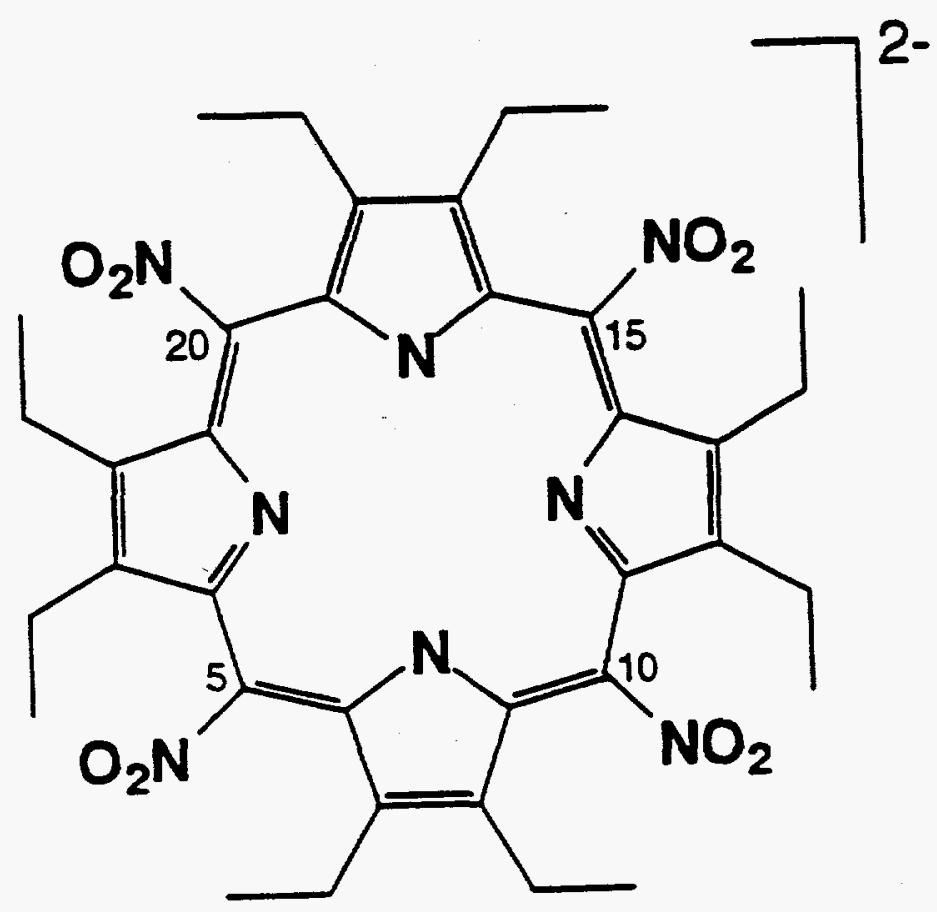




\subsection{CATALYTIC CONVERSION OE LIGHT ALKANES - PROOF OF CONCEPT STAGE}

\subsection{OBJECTIVES}

The objective of the work presented in this paper is to develop new, efficient catalysts for the selective transformation of the light alkanes in natural gas to alcohols for use as liquid transportation fuels, fuel precursors and chemical products. There currently exists no direct catalytic air-oxidation process to convert these substrates to alcohols. Such a one-step route would represent superior useful technology for the utilization of natural gas and similar refinery-derived light hydrocarbon streams. Processes for converting natural gas or its components (methane, ethane, propane and the butanes) to alcohols for use as motor fuels, fuel additives or fuel precursors would not only add a valuable alternative to crude oil but would produce a clean-burning, high octane oxygenate which would be a major component of reformulated gasoline (RFG).

\subsection{BACKGROUND INFORMATION}

Natural gas is an abundant resource that can provide an inexpensive feedstock for production of $\mathrm{C}_{1}-\mathrm{C}_{4}$ alcohols. The low molecular weight alcohols, methanol, ethanol, isopropyl alcohol and tert-butyl alcohol have seen application both as alternative fuels and in reformulated gasoline. The ethers that are made from these alcohols: methyl-tert-butyl ether, MTBE, ethyl-tert-butyl ether, ETBE, diisopropyl ether,DIPE, and tert-amyl methyl ether, TAME, are major oxygenate components of RFG. These oxygenates have high blending value octane numbers, BVON, and good gasoline properties. Of these, MTBE has seen the most widespread use and the most rapid growth in RFG.

Working with ideas generated from recent understanding of biological systems responsible for enzymatic oxidations, Sun has developed a number of new catalyst systems for the direct oxidation of light alkanes to alcohol-rich oxygenate mixtures (1-40). The relative ease of oxidation of natural gas components with our catalyst systems has been found to be: isobutane>propane> ethane>methane. For this reason, the first oxidation reaction to reach the proof-of-concept stage was the oxidation of isobutane to TBA. TBA has been used as a high-octane fuel component and is the major precursor to MTBE. MTBE has excellent fuel properties. Among these are the apparent reduction of CO emissions from automotive exhaust. MTBE is currently the oxygenate of choice for RFG.

Field butanes have historically found a large number of liquid fuel precursor applications. Traditionally, this $\mathrm{C}_{4}$ component was blended into gasoline for octane and vapor pressure. Light hydrocarbons, however, whether from natural gas or petroleum refining are rejected in large part from the gasoline pool in response to environmental regulations of vapor pressure emissions. After isomerization, the isobutane can be dehydrogenated to isobutylene which is a precursor to alkylate and MTBE - both high octane, clean-burning RFG components. Dehydrogenation is costly, however. Direct oxidation of isobutane to TBA is a way to avoid this expensive process step. The TBA produced is a viable high octane fuel oxygenate and a precursor to MTBE as well. Sun catalytic technology, therefore is able to convert a $\mathrm{C}_{4}$ stream into a fuel oxygenate without requiring a costly dehydrogenation step. 
Thus, in summary, the direct reaction of a light alkane with air or oxygen to give an alcohol-rich oxidate would: a) utilize an inexpensive feedstock unacceptable for liquid transportation fuel use, b) produce a material that would have superior gasoline performance properties such as driveability and high octane, and c) produce a clean-burning alternative to conventional gasoline for use in areas that fail to meet air quality standards.

\subsection{PROJECT DESCRIPTION}

During the stage at which this work ended, our program consisted of two phases which were running concurrently, a research phase and a proof-of-concept phase. The function of the research phase is to design synthesize and perform laboratory tests on new materials in order to enhance the catalytic properties of the suprabiotic systems generated in our laboratories. Increases in catalytic activity and process selectivity as well as extensions of structure-activity relationships are examined. The research phase is intended to provide the process development phases with catalysts having superior properties for light alkane oxidation. The second concurrent phase, is the proof-of-concept phase. In this phase process development of oxidations which have succeeded in the research phase are carried out in a pre-pilot PDU. At the conclusion of the cooperative agreement we were developing a process for the conversion of isobutane to TBA. We have identified two routes for this transformation: a one-step route directly from isobutane to TBA, and a two-step route in which the isobutane is oxidized first to tert-butyl hydroperoxide and then transformed into TBA catalytically. This section will summarize the results of these activities. Details of the proof-of-concept stage are given in the fifth Topical Report on the Cooperative Agreement.

\subsection{RESULTS}

One Step Oxidation of Isobutane to TBA. There are currently no efficient catalysts for the direct oxidation of isobutane to TBA, eq.

1. Cobalt salts and complexes have been used

$$
\text { i- } \mathrm{C}_{4} \mathrm{H}_{10}+1 / 2 \mathrm{O}_{2}-->\mathrm{t}-\mathrm{C}_{4} \mathrm{H}_{9} \mathrm{OH}
$$

(41) but in order to achieve good rates, high catalyst concentrations are needed as well as high temperature. Under these conditions poor selectivity is achieved. In the research phase of our work we have designed, synthesized, and tested a family of metal complexes that are very effective catalysts for the selective low temperature oxidation of isobutane to TBA. These catalysts, the perhaloporphyrinatometal complexes, were designed based on biomimetic considerations. The halogen ligands on the periphery of the porphyrin ring withdraw electrons from the metal center, increase the $M(\mathrm{III}) / \mathrm{M}(\mathrm{II})$ reduction potential and enhance the alkane oxidation activity. We have further shown that iron perhaloporphyrins have the greatest activity of any of the metalloporphyrins studied. Figure 1 shows that as the degree of electron withdrawal (number of halogens on the porphyrin macrocycle) increases, the reduction potential of a series of iron porphyrin complexes increases and the activity of the catalysts steadily increases. Table 1 shows that these 
perhaloporphyrin catalysts are effective soluble catalysts in neat isobutane and give unprecedented rates and selectivities for converting isobutane to TBA.

The same iron perhaloporphyrin complexes that were found to be effective for the direct oxidation of isobutane to TBA were also shown to be excellent catalysts for the decomposition of t-butylhydroperoxide, TBHP, to TBA, Figure 2, Table 2. Again, perhaloporphyrin complexes were over an order of magnitude more active than previously known catalysts. The same relationship was found between $\mathrm{Fe}$ (III)/Fe(II) reduction potential as in the direct oxidation reaction, Table 3 . Since TBHP is made commercially by non-catalytic oxidation of isobutane (42), our work suggested the possibility of a competitive two-step route from isobutane to TBA, eq. 2,3 .

$$
\begin{aligned}
& \mathrm{i}-\mathrm{C}_{4} \mathrm{H}_{10}+\mathrm{O}_{2}-->{ }_{\mathrm{t}}-\mathrm{C}_{4} \mathrm{H}_{9} \mathrm{OOH} \\
& \mathrm{t}-\mathrm{C}_{4} \mathrm{H}_{9} \mathrm{OOH} \quad-->\mathrm{t}-\mathrm{C}_{4} \mathrm{H}_{9} \mathrm{OH}
\end{aligned}
$$

Since both of these reactions work so well, we determined to proceed with evaluating the concept of potentially commercial routes from isobutane to TBA using both the one-step and the two-step processes. Together with a joint R\&D partner we set proprietary hypothetical commercial targets for each process. The concept was proven, therefore, if our catalytic processes met these criteria. We first examined the direct one-step oxidation route. Our process concept is shown schematically in Figure 3. Using our best perhaloporphyrin complex as a catalyst we met all targets except that of catalyst cost. Efforts using new catalysts with lower costs failed to meet rate and selectivity targets. Efforts to lower the cost of manufacture of the perhaloporphyrin complexes failed to achieve costs that were even close to what was needed. When the project ended we had found a way to make the inexpensive, first row metals including iron, far more active for oxidation of an alkane, than had ever been observed. How to do this with a low-cost ligand system is the one remaining technical problem with the one-step route.

Figure 4 shows a schematic flow diagram of the proposed two step process for manufacture of TBA. Catalyst cost again was a problem for the iron perhaloporphyrins. In this case, however we were able to find proprietary new catalyst systems that met the catalyst cost criterion. At the conclusion of the cooperative agreement we had met or exceeded all of the performance criteria for a two-step process. Table 4 summarizes our progress vs goals up until the end of the project in October, 1994

\subsection{CONCLUSIONS}

During the period of the cooperative agreement we have uncovered a family of catalysts which have shown unprecedented activity for the oxidation of alkanes to alcohol-rich oxidates. These catalysts have a ligand system which confers unusually high oxidation activity to inexpensive first row transition metals, especially iron. If less expensive analogs can be produced, they should ultimately have great practical value in converting natural gas components to fuel oxygenates. Isobutane can be converted to TBA in excellent selectivity at acceptable rates for a practical process if catalyst cost can be lowered. In the case of the conversion of isobutane to TBA we have found a two step route through the hydroperoxide that could have value in a retrofit of an existing plant. 


\subsection{REFERENCES}

(1) P. E. Ellis, Jr. and J. E. Lyons, I. Chem. Soc. Chem. Commun., (1987) 1187.

(2) P. E. Ellis, Jr. and J. E. Lyons, I Chem. Soc Chem Commun., (1989) 1189.

(3) P. E. Ellis, Jr. and J. E. Lyons, J Chem. Soc. Chem. Commun., (1989) 1315.

(4) P. E. Ellis, Jr. and J. E. Lyons, Catalysis Letters $\underline{3}$, (1989) 389.

(5) P. E. Ellis, Jr. and J. E. Lyons, Preprints of the Division of Petroleum Chemistry, ACS, Vol. 35, No. 2, April (1990).

(6) P. E. Ellis, Jr. and J. E. Lyons, Coordination Chem. Rev., 105 (1990) 181.

(7) J. E. Lyons and P. E. Ellis, Jr., Catalysis Letters, 8 (1991) 45.

(8) J. E. Lyons and P. E. Ellis, Jr., Applied Catalysis A General, 84 (1992) L1-L6.

(9) J. E. Lyons, P. E. Ellis, Jr., R. W. Wagner, P. B. Thompson, H. B. Gray, M. E. Hughes and J. A. Hodge, Preprints Pet. Div., $\underline{37}$ (1992) 307.

(10) J. E. Lyons, "Catalytic Conversion of Light Alkanes," GRI Contractor Review Meeting, Methane Reaction Science Program, Paris, France, Aug. 25-27 (1991).

(11) Unpublished Work by H. B. Gray, W. P. Schaefer, et al.

(12) Related structures have been determined: a) R. E. Marsh, W. P. Schaefer, J. A. Hodge, M. E. Hughes, H. B. Gray, J. E. Lyons and P. E. Ellis, Jr., Acta Cryst. Sec. C, V 49 (1993) 1339. b) W. P. Schaefer, J. A. Hodge, M. E. Hughes, H. B. Gray, J. E. Lyons, P. E. Ellis, Jr. and R. W. Wagner, Acta Cryst. Sec. C, V 49 (1993) 1342.

(13) J. E. Lyons, P. E. Ellis, Jr. and V. A. Durante, Proceedings of the Natural Gas R\&D Contractors Review Meeting, DOE/METC - 89/6103 (1989) 164.

(14) J. E. Lyons, Proc Nat. Gas R\&D Contractors Review Meeting, DOE/METC - 91/6117 (1990) 276.

(15) J. E. Lyons, P. E. Ellis, Jr., H. K. Myers, Jr., G. Suld and W. A. Langdale, U. S. Patent $4,803,187$, Feb. 7 (1989).

(16) P. E. Ellis, Jr. and J. E. Lyons, U. S. Patent 5,091,354, Feb. 25 (1992). 


\subsection{REFERENCES}

(CONTINUED)

(17) J. E. Lyons, P. E. Ellis, Jr., W. A. Langdale and H. K. Myers, Jr., U. S. Patent 4,916,101, Apr. 23 (1990).

(18) P. E. Ellis, Jr. and J. E. Lyons, U. S. Patent 4,898,989, Feb. 6 (1990).

(19) J. E. Lyons, P. E. Ellis, Jr., H. K. Myers, Jr., G. Suld and W. A. Langdale, U. S. Patent 4,859,798, Aug. 22 (1989).

(20) J. E. Lyons, Proc. Nat Gas R\&D Review Meeting, DOE/METC - 92/6125 (1992) 266.

(21) J. E. Lyons, P. E. Ellis, Jr. and V. A. Durante, Studies in Surface Science and Catalysis, 67, Elsevier, N.Y. (1991) 99.

(22) P. E. Ellis, Jr. and J. E. Lyons, U. S. Patent 5,254,740, Oct. 19 (1993).

(23) P. E. Ellis, Jr. and J. E. Lyons, U. S. Patent 5,212,300, May 18 (1993).

(24) P. E. Ellis, Jr. and J. E. Lyons, U. S. Patent 5,120,882, June 9 (1992).

(25) P. E. Ellis, Jr. and J. E. Lyons, U. S. Patent 5,118,886, June 2 (1992.

(26) P. E. Ellis, Jr., J. E. Lyons and H. K. Myers, Jr., U. S. Patent 5,093,491, Mar. 3 (1992).

(27) P. E. Ellis, Jr. and J. E. Lyons, U. S. Patent 4,970,348, Nov. 13 (1990).

(28) P. E. Ellis, Jr., J. E. Lyons and H. K. Myers, Jr., U. S. Patent 4,900,871, Feb. 13 (1990).

(29) P. E. Ellis, Jr., J. E. Lyons and H. K. Myers, Jr., U. S. Patent 4,895,682, Jan. 23 (1990).

(30) P. E. Ellis, Jr., J. E. Lyons and H. K. Myers, Jr., U. S. Patent 4,895,680, Jan. 23 (1990).

(31) J. E. Lyons, P. E. Ellis, Jr., H. K. Myers, Jr., and R. W. Wagner, J. Catal., 141 (1993) 311.

(32) J. E. Lyons and P. E. Ellis, Jr., U.S. Patent 5,120,886, June 9 (1992).

(33) P. E. Ellis, Jr., and J. E. Lyons, "Metal Phthalocyanine Oxidation Catalysts," U.S. patent 5,254,740, issued October 19, 1993.

(34) P. E. Ellis, Jr., and J. E. Lyons, "Nitrated Metalloporphyrins as Catalysts for Alkane Oxidation," U.S. Patent 5,280,115, issued January 20, 1994. 


\subsection{REFERENCES}

(CONTINUED)

(35) J. E. Lyons and P. E. Ellis, Jr., "Decomposition of Organic Hydroperoxides with Nitrated Porphyrin Complexes, U.S. Patent 5,345,008, issued September 6, 1994.

(36) P. E. Ellis, Jr. and J. E. Lyons, "Metal Phthalocyanine Catalysts", U.S. Patent 5,354,857, issued October 11, 1994.

(37) P. E. Ellis, Jr. and J. E. Lyons, "Cyano- and Polycyanometalloporphyrins as Catalysts for Alkane Oxidation," U.S. Patent 5,382,662, Issued January 7, 1995.

(38) P. E. Ellis, Jr., J. E. Lyons and S. N. Shaikh, "Novel Iron meso-Nitroporphine Complexes for the Catalytic Reaction of Alkanes with Molecular Oxygen," Catal. Lett., 24, 79 (1994).

(39) H. L. Chen, P. E. Ellis, Jr., T. P. Wijesekera, T. E. Hagan, S. E. Groh, J. E. Lyons and D. P. Ridge, "Correlation Between Gas Phase Electron Affinities, Electrode Potentials and Catalytic Activities of Halogenated Metalloporphyrins", I. Amer. Chem. Soc., 116, 1086 (1994).

(40) J. E. Lyons and P. E. Ellis, Jr., Reactions of Alkanes with Dioxygen: Toward Suprabiotic Systems, Metalloporphyrins in Catalytic Oxidations, R. S. Sheldon, Ed. Marcel Dekker, N.Y., pp 297-324 (1994).

(41) D. E. Winkler and G. W. Hearne, Ind. and Eng. Chem., 33 , 655 (1961).

(42) P. D. Taylor and M. T. Mocella, U.S. Patent 4,508,923 (1985). 
Table 1. Iron Haloporphyrin - Catalyzed Isobutane Oxidation

Charge to Reactor Conversion Select

\begin{tabular}{|c|c|c|c|c|c|c|c|}
\hline Catalyst & I. ${ }^{\circ} \mathrm{C}$ & I. Hrs & $\dot{i C_{1}} \mathrm{H}_{3 \sigma}$ & $\underline{Q}_{2}$ & ${ }_{i-C_{1}} H_{10} \%$ & TBA\% & TONc \\
\hline \multirow{4}{*}{$\mathrm{Fe}\left(\mathrm{TPPF}_{20} \mathrm{~B}-\mathrm{Br} \mathrm{r}_{8}\right) \mathrm{Cl}$} & 80 & 3 & 1870 & 53 & 17 & 87 & 10,660 \\
\hline & 80 & 3 & 1862 & 100 & 28 & 83 & 17,150 \\
\hline & 80 & 3 & 1865 & 47 & 14 & 91 & 8,420 \\
\hline & 25 & 71.5 & 1862 & 53 & 22 & 92 & 13,560 \\
\hline $\mathrm{Fe}\left(\mathrm{TPPF}_{20}\right) \mathrm{OH}$ & 24 & 143 & 1871 & 53 & 18 & 95 & 12,150 \\
\hline
\end{tabular}

Table 2. Conversion of TBHP to TBA

\begin{tabular}{|c|c|c|c|c|c|}
\hline \multirow[b]{2}{*}{ Catalyst } & \multirow{2}{*}{$\begin{array}{l}\text { Time } \\
\text { Hours }\end{array}$} & \multirow{2}{*}{$\begin{array}{l}\mathrm{E}-\mathrm{BuO} \mathrm{O}_{2} \mathrm{H} \\
\text { Conv. \% }\end{array}$} & \multicolumn{3}{|c|}{ Product, Molar Sel., \% } \\
\hline & & & $\mathrm{E}-\mathrm{BUOH}$ & $(t-B \cup O)_{2}$ & $\left(\mathrm{CH}_{2}\right)_{2} \mathrm{CO}$ \\
\hline $\mathrm{Fe}(\mathrm{ACAC})_{3}$ & 2.3 & $<5$ & 67 & $\operatorname{tr}$ & 32 \\
\hline $\mathrm{Fe}(\mathrm{TPP}) \mathrm{Cl}$ & 1.9 & 27 & 82 & 7 & 11 \\
\hline $\mathrm{Fe}\left(\mathrm{TPPF}_{20}\right) \mathrm{Cl}$ & 3.3 & 72 & 87 & 10 & 3 \\
\hline $\mathrm{Fe}\left(\mathrm{TPPF}_{20} \mathrm{~B}-\mathrm{Br} r_{8}\right) \mathrm{Cl}$ & 1.9 & 95 & 90 & 8 & 2 \\
\hline $\mathrm{Fe}\left(\mathrm{TPPF}_{20} \mathrm{~B}-\mathrm{Cl}_{8}\right) \mathrm{Cl}$ & 3.3 & 100 & 90 & 8 & 2 \\
\hline
\end{tabular}

Table 3. Electron Affinity Reduction Potential and Catalytic Activity

\begin{tabular}{|c|c|c|c|c|}
\hline Species & $E A(e V)$ & $\begin{array}{c}E_{1 / 2}(V) \\
V S . S C E\end{array}$ & $\begin{array}{c}\text { TO } \\
\text { ic }-0 x\end{array}$ & $\begin{array}{l}\text { TBA- Yield } \\
\text { TBHP-dec }\end{array}$ \\
\hline $\mathrm{Fe}(\mathrm{TPP}) \mathrm{Cl}$ & 2.15 & -.29 & 0 & 4.8 \\
\hline $\mathrm{Fe}\left(\mathrm{TPPF}_{20}\right) \mathrm{Cl}$ & 3.14 & +.08 & 1160 & 16.0 \\
\hline $\mathrm{Fe}\left(\mathrm{TPPF}_{20} \mathrm{BCl}_{8}\right) \mathrm{Cl}$ & 3.35 & +.29 & 1800 & 67.2 \\
\hline
\end{tabular}




\section{Table 4. \\ Isobutane to TBA Proof-of-Concept Criteria (New Retrofit Process Modes)}

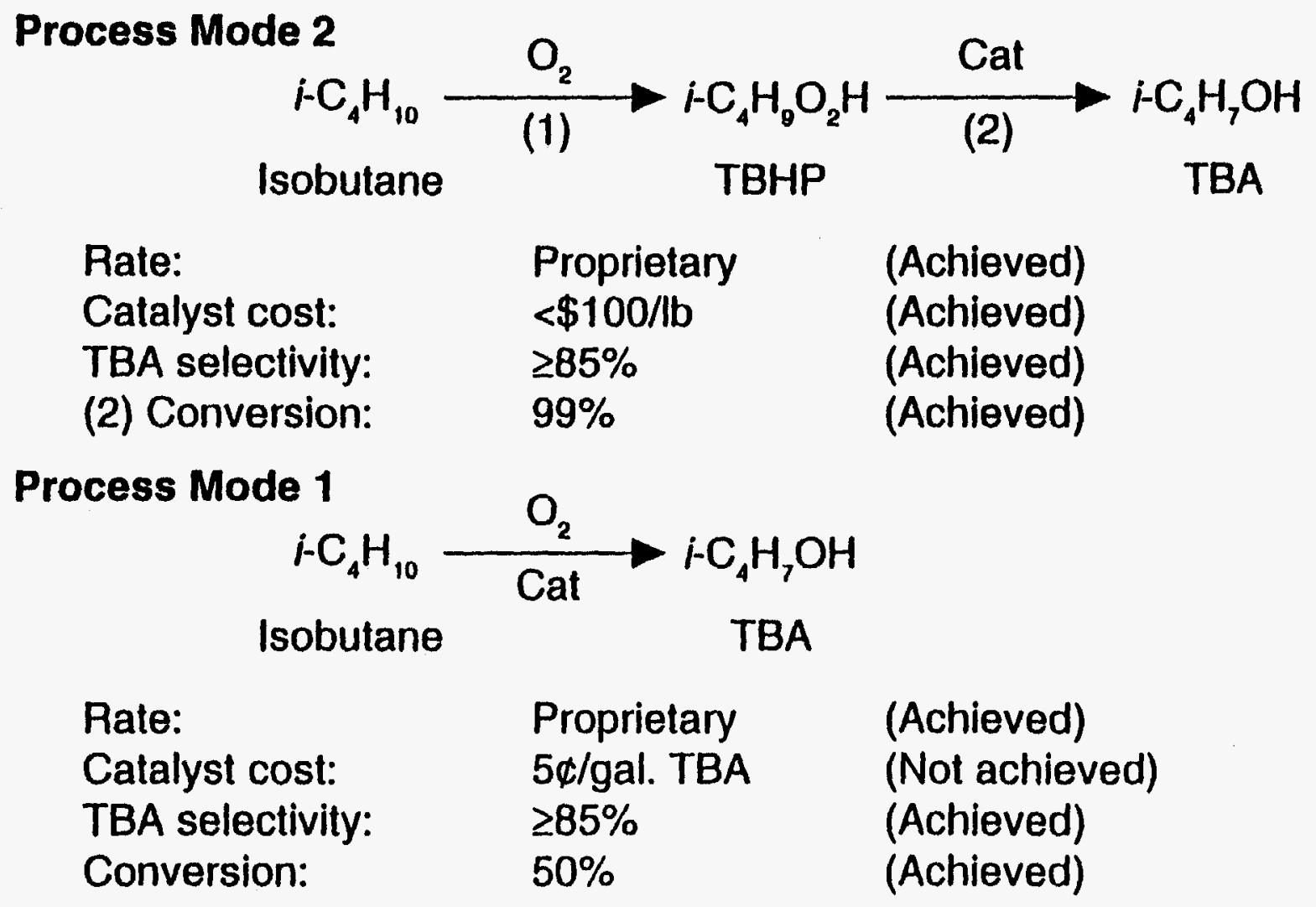

Process Mode 1

$$
i-\mathrm{C}_{4} \mathrm{H}_{10} \underset{\mathrm{Cat}}{\stackrel{\mathrm{O}_{2}}{\longrightarrow}} \mathrm{i}-\mathrm{C}_{4} \mathrm{H}_{7} \mathrm{OH}
$$

Isobutane

TBA

Rate:

Catalyst cost:

TBA selectivity:

Conversion:

Proprietary
$5 \notin /$ gal. TBA
$\geq 85 \%$
$50 \%$

(Achieved)

(Not achieved)

(Achieved)

(Achieved) 
Figure 1. Catalyst Activity vs. Redox Potential
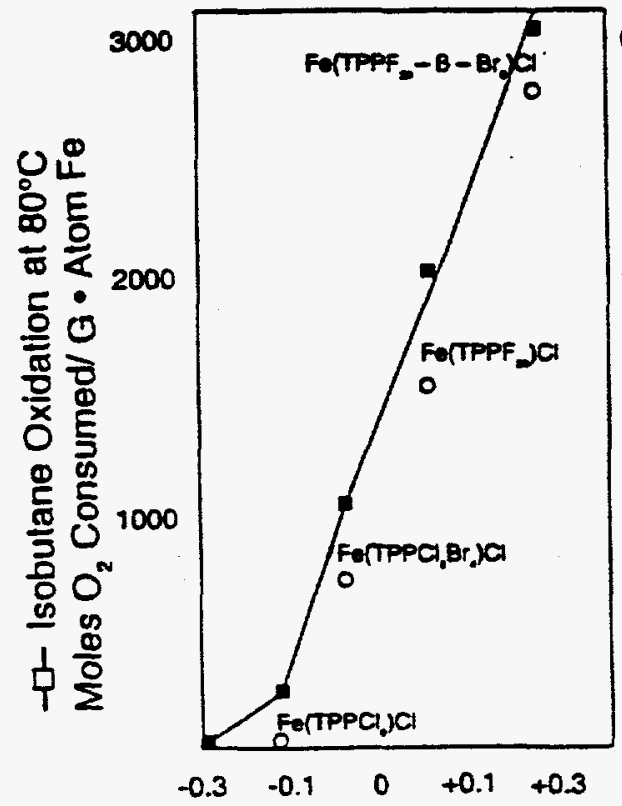

Reduction Potential $\mathrm{Fe}^{3+p+}$ vs. SCE
Figure 2. TBA from TBHP

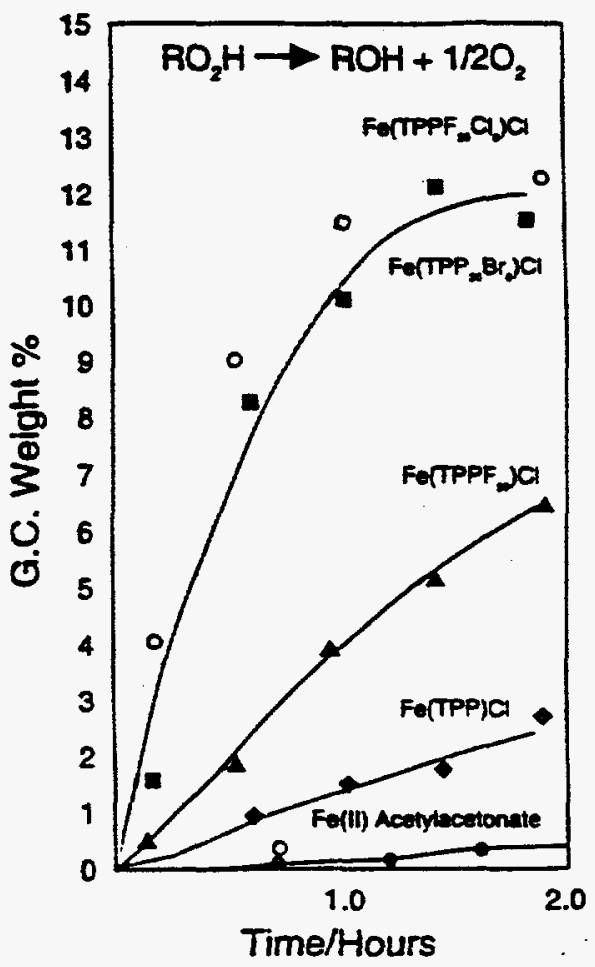

Figure 3. Isobutane Oxidation - One Step Process

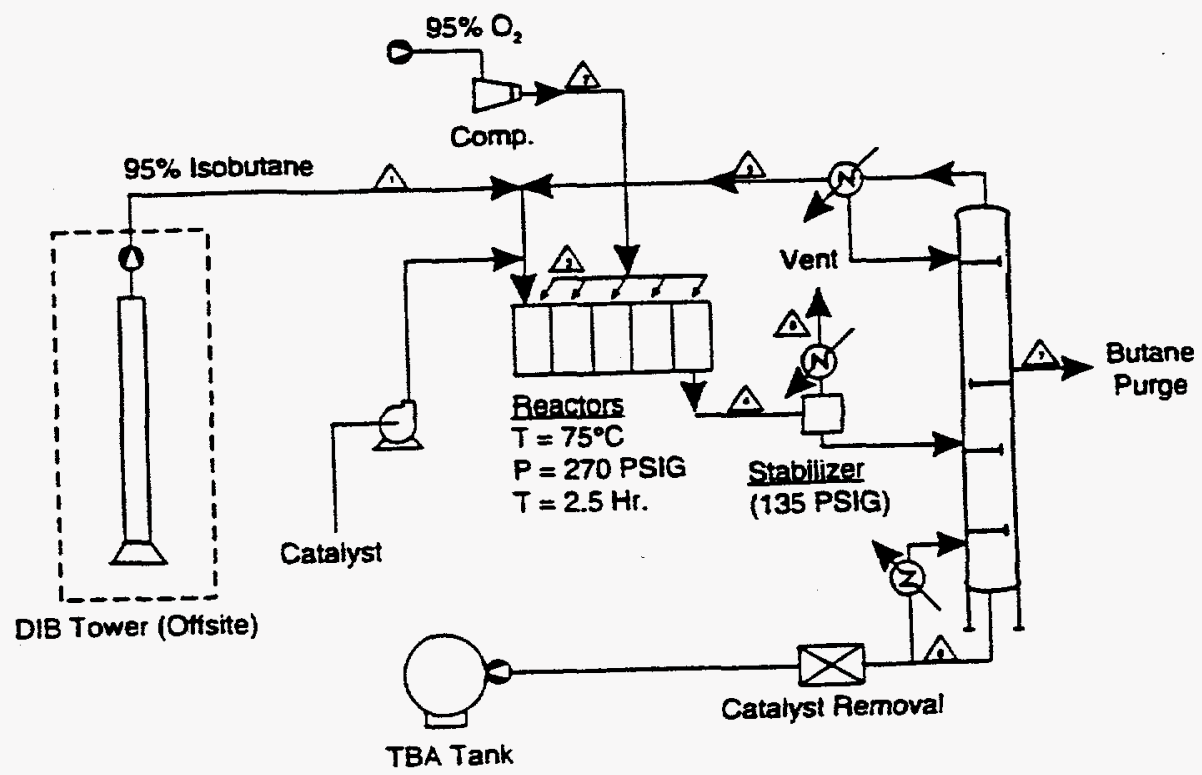


Figure 4. Isobutane Oxidation - Two Step Process
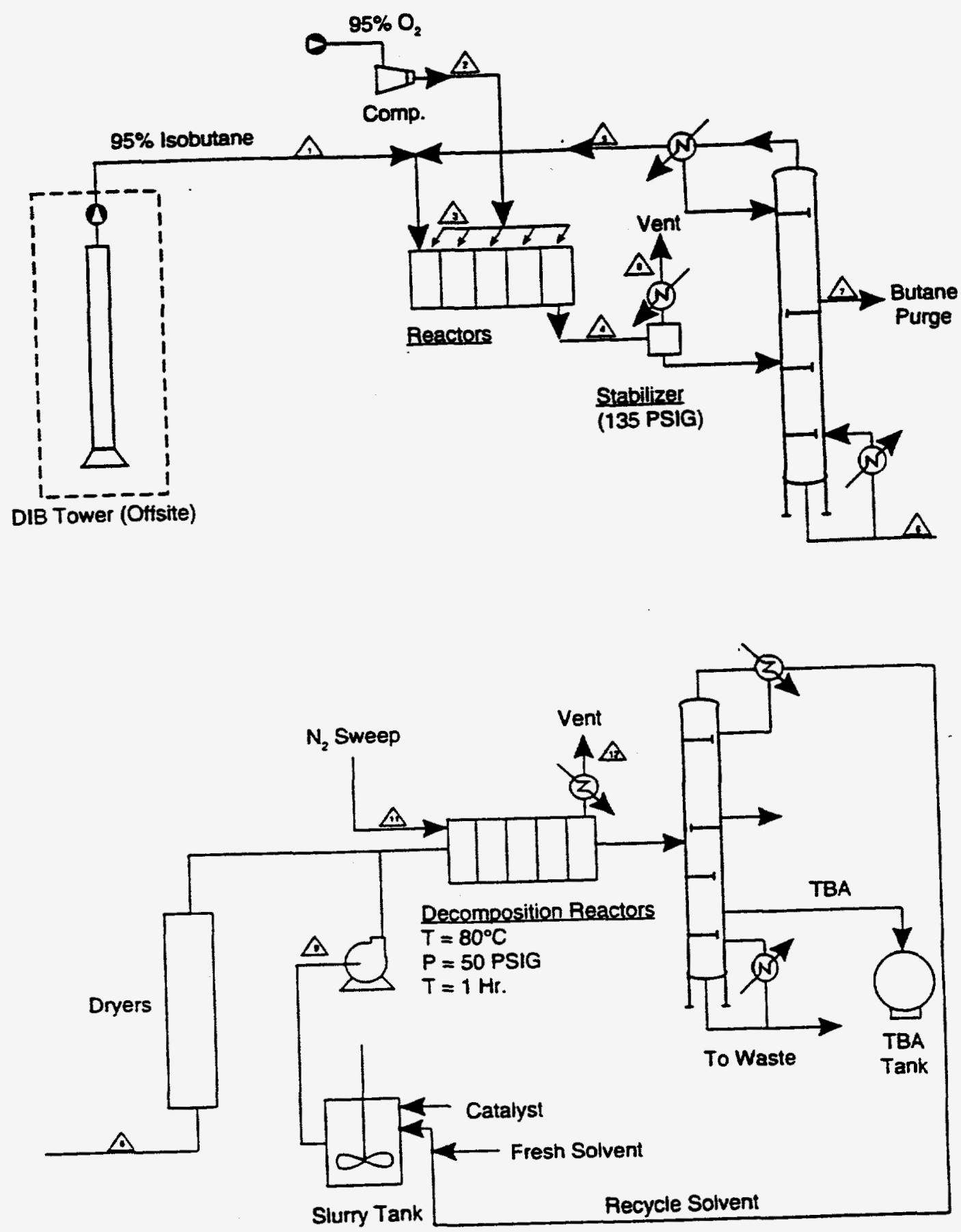


\subsection{HETEROGENEOUS CATALYSTS FOR LIGHT ALKANE OXIDATION}

\subsection{SUMMARY}

Progress has been made in four major areas of this work. First, a superior kinetic model was developed to determine the rates, yields and selectivities for methanol formation from methane by gas phase autoxidation. It was essential to know what to expect from homogeneous gas phase oxidations both as a base-line for catalytic studies and to assess literature claims relative to a potential new methane-to-methanol process.

Secondly, catalysts capable of converting methane to methanol were designed synthesized and characterized. Thirdly, suitable reactor systems were designed, built, and lined out as catalyst test units. Finally, the catalytic materials were tested for their activity on methane, ethane, and mixed light gases.

While we have achieved considerable success and have generated the best available catalysts for oxidizing methane directly to methanol, additional design criteria must be incorporated in order to produce materials capable of reaching commercially acceptable targets. We have identified these deisgn criteria and were proceeding to synthesize superior catalysts system at the close of the program in October, 1994.

\subsubsection{Methane Oxidation Kinetic Model}

We developed a kinetic model $(1-14)$ to determine the conditions at which methane and light alkanes oxidize and the expected products as a function of reaction conditions. Details of this model can be found in the fourth Topical Report of the Cooperative Agreement.

We have kinetically modelled vapor phase reactions which are uncatalyzed and those in which gas phase radicals are catalytically produced by homolytic $\mathrm{C}-\mathrm{H}$ bond cleavage over solid oxidic surfaces. Increased methanol selectivity up to about $65 \%$ at $~ 10 \%$ conversion is achieved by catalysis of methyl radical generation. Higher yields of methanol will require participation of a non-radical pathway. Kinetic modelling, therefore, seems to indicate that significant contributions from a selective non-radical pathway are probably required for methanol selectivity over $70 \%$.

\subsubsection{Catalyst Synthesis Characterization and Testing}

We synthesized, characterized and tested new zeolite catalysts which have iron substituted into their siliceous frameworks for vapor phase oxidation of methane to methanol. We have found that the higher the iron content of a framework-substituted zeolite the higher is its catalytic activity for methanol formation. In addition, we have discovered that in a series of iron sodalites, catalysts which have iron in BOTH the framework AND in the proximate extraframework exchange sites are the most active of the iron sodalite catalysts that we have tested. Using these catalysts in a reactor designed to limit contact of products and intermediates with the surface, we produce methanol in up to $70 \%$ 
selectivity at $7 \%$ conversion. This is the the most productive direct catalytic synthesis of methanol from methane that we are aware of.

We have also studied the oxidation of ethane over iron exchanged iron sodalite, Fex[Fe]SOD, and compared these results to the known uncatalyzed gas phase oxidation of ethane reported by $\mathrm{H}$. Gesser. Using our active iron sodalite catalyst we have achieved carbon selectivities to liquid oxidate of from $40-60 \%$ at ethane conversions of from $6-11 \%$ at temperatures below 300 . Methanol/ethanol ratios of from 3-9 were observed. Using the molar selectivity calculation of Gesser our selectivities range from $40-70 \%$ and seem comparable with his results under similar conditions of temperature and pressure. We would expect to operate at lower temperature with a catalyst but iron sodalite does not allow us to go below the thermal $\left(\sim 250^{\circ} \mathrm{C}\right)$ oxidation range.

We have prepared and characterized a series of new catalysts which have iron in the framework of the sodalite structure and either iron, chromium or manganese in the exchange site. In addition to substituting iron, we have also attempted to get chromium and manganese into the sodalite framework. We have managed to get only small amounts of chromium into the framework and have failed in many attempts to make a manganese sodalite. We have made framework-substituted pentasil zeolites containing iron, manganese, chromium and ruthenium. The amount of framework metal which will be accepted by the zeolite is low however - generally less than $3 \%$. We have tested the iron exchanged pentasil zeolite for methane oxidation and found that its activity was quite a bit inferior to sodalite which had a high concentration of framework iron. We also synthesized tested catalysts which have chromium oxo centers incorporated into surface oxidic layers. Silica surfaces containing discrete chromium oxo units were catalytically active for methane oxidations.

During the period of this project, we were also able to generate for the first time an active and selective methane oxidation catalyst having manganese in the framework of a zeolitic material. In past reports we have disclosed that framework substituted iron sodalite can be an active and relatively selective catalyst for converting methane to methanol. All attempts to incorporate large amounts of manganese into the sodalite framework failed. We further found that iron-exchanged, iron substituted sodalite was a superior oxidation catalyst to manganese-exchanged, iron substituted sodalite. Supported manganese catalysts such as manganese oxides on silica or alumina largely gave combustion to carbon oxides with little or no methanol formed.

We have now found a new and as yet uncharacterized crystalline aluminophosphate phase containing over $8 \%$ manganese, MnAPO-X, which catalyzes selective oxidation of methane producing significant amounts of methanol below $400^{\circ} \mathrm{C}$. At temperatures below $400^{\circ} \mathrm{C}$ the new manganese catalyst is at least as active as iron sodalite and produces methanol with equivalent selectivity. We have also prepared an unknown iron aluminophosphate by an analogous preparation and will test its catalytic activity. To our knowledge this class of crystalline aluminophosphates have not been used to oxidize methane under conditions which give high methanol selectivity.

Methanol had also been formed in good selectivity at conversion levels close to those obtained for the MnAPO-X using iron sodalite catalysts. Because of the manganese results we were encouraged to prepare and test an aluminophosphate catalyst containing large amounts of framework exchanged iron. We prepared the known FAPO-11 phase and tested it under mild conditions. It was 
not nearly as active as MnAPO-X nor was methanol formed in as high selectivity. It should be noted, however, that the structure of MnAPO-X is quite different than that of FAPO-11.

We have also made MnAPO-5, and CoAPSO-5 and compared these materials with highly pure $\mathrm{Fe}[\mathrm{SOD}]$. The former compounds are aluminophosphate stuctures into whose framework manganese and cobalt are substituted. As we have reported in the past, $\mathrm{Fe}$ [SOD] does not have activity because of the difficulty of incorporating $\mathrm{Fe}$ (II) into the sodalite structure. The effective $\mathrm{Fe}$ (III)/Fe(II) reduction potential, therefore, will be low. When $\mathrm{Fe}(\mathrm{II})$ is incorporated into the exchange sites an active catalyst: $\mathrm{Fe}_{x} \mathrm{Fe}$ [SOD] is prepared. In the case of both MnAPO-5 and CoAPSO-5, the metals may be incorporated in both the (II) and (III) oxidation states. This is important since oxygen will be bound and hopefully activated by $\mathrm{Mn}$ (II) and $\mathrm{Co}(\mathrm{II})$. We have tested MnAPO-5 for oxidation of both methane and ethane. This catalyst is more active than $\mathrm{Fe}_{\mathrm{x}}[\mathrm{Fe}] \mathrm{SOD}$ and nearly as selective. Using MnAPO-5 we can homolyze the $\mathrm{C}-\mathrm{H}$ bond of methane at temperatures fully $35^{\circ} \mathrm{C}$ lower than when $\mathrm{Fe}_{\mathrm{x}}[\mathrm{Fe}] \mathrm{SOD}$ is used as catalyst.

Thus, we have identified three important criteria for a methane to methanol catalyst: stability under reaction conditions (2) proximate metal centers to activate dioxygen and (3) high reversible metal redox potential. Two other criteria were identified and will be necessary for higher methanol selectivity- geometric facilitation of "rebound" of methyl radicals to form methanol on the surface and hydrophobicity to release methanol rapidly from the surface as formed.

We completed a study on the effect of reactor design on the selectivity of iron sodalite catalyzed oxidation of methane to methanol. We find that a complex design having a combination of a low-temperature back-mixed zone over a hot catalyst coupled with reactive distillation gives methanol in $70 \%$ selectivity at up to $7 \%$ methane conversion (oxygen conversion $>90 \%$ ). As we have mentioned in past reports, this system is too complex to extract unambiguous catalyst screening data. We have constructed three simpler systems which separate the catalytic activity from the reactive distillation aspects. One system operates as a simple downflow reactor in the plug flow mode. In this system under conditions of temperature and pressure comparable to those used in the complex reactor, iron sodalite catalyzes methane oxidation to methanol in $50 \%$ selectivity. Another system which operates in a the back-mixed mode gives methanol in up to $30 \%$ selectivity at similar temperature, pressure and flow rate. Clearly, reactor design is critical in conducting these oxidations and was closely scrutinized. We concluded that, while simple downflow reactor systems are not designed to optimize methanol productivity, they are probably the most straightforward way to qualitatively differentiate catalytic efficiency. For this reason we have adopted this technique for screening catalysts at the present time.

\subsection{CATALYST SYNTHESIS}

Surface oxide catalysts were prepared that aimed at incorporating oxidation-active transition metal centers in a stable oxidic lattice environment such that proximate metal centers could reductively bind and cleave the oxygen molecule to generate active $M O$ centers for vapor phase oxidation of methane, ethane and other light alkanes. Among the catalysts successfully prepared for testing were iron sodalites, metal framework - substituted pentasil zeolites, iron, manganese and cobalt framework substituted aluminophosphates and surface bound chromium oxo catalysts. 
We are attempting to use the framework of crystalline aluminosilcates and aluminophosphates as ligand systems for oxidation-active first row transition metals which could have redox properties similar to those of the porphyrin or polyoxometalate species described previously yet would be suitable for use as vapor phase catalysts for methane or ethane oxidations at high temperature. Low oxidation activity of early framework-substituted zeolites has been interpreted as due to apparently low (Fe(III)/Fe(II) reduction potentials which may have been due to inaccessibility of important oxidation states. We, therefore, concentrated on the synthesis, characterization and testing of framework-substituted aluminophosphates with the belief that stability of the $M(I I)$ oxidation state may be higher in these catalysts and the hope that $\mathrm{Fe}$ (III)/Fe(II) redox process may be more facile within their frameworks. We have prepared and characterized MnAPO-5>47, CoAPSO-5>47, MnAPO-5, MnAPO-11, and FAPO-11 and have tested their oxidation activity.

\subsubsection{Iron Sodalite}

A silicoferrate, iron sodalite, was synthesized by a modification of the method of Szostak and Thomas supra. A solution of $500 \mathrm{~g}$. sodium silicate solution (Fisher Scientific) and $108 \mathrm{~g}$. sodium hydroxide in $200 \mathrm{~g}$. deionized water was prepared and designated solution A. A second solution, solution B, was prepared by adding $82 \mathrm{~g}$. of $98 \%$ sulfuric acid and $80.4 \mathrm{~g}$ of iron (III) nitrate nonahydrate (Aldrich) to $200 \mathrm{~g}$. deionized water with stirring. Solution A and solution B were mixed by 25 alternate addition of small aliquots of each to a beaker fitted with an overhead stirrer. Solution $\mathrm{C}$ was prepared by adding $82.7 \%$ tetramethylamonium chloride (Aldrich) to $137 \mathrm{~g}$. deionized water.

Solution $\mathrm{C}$ was then rapidly added to the mixture of $\mathrm{A}$ and $\mathrm{B}$ with vigorous stirring. The resulting tan slurry had a relative molar composition ratio of $1.0 \quad \mathrm{Fe}_{2} \mathrm{O}_{3}: 24.2 \mathrm{SiO}_{2} 20.7 \mathrm{Na}_{2} \mathrm{O}$ : 7.6TMACl: $465 \mathrm{H}_{2} \mathrm{O}$ and a $\mathrm{pH}$ of 11.5 . The slurry was stirred without additional cooling for 15 minutes then charged to a Teflon lined 2 liter autoclave, sealed, and purged with argon. The reactor was pressurized to 200 psig with argon and allowed to crystallize with stirring at $168^{\circ}-172^{\circ} \mathrm{C}$. for 68 hours. The reaction product was washed with 1 liter of hot distilled water and 3 liters of room temperature distilled water and dried for 2 days at $125^{\circ} \mathrm{C}$ in air. The recovered dried product $(80.3 \mathrm{~g}$.) was then calcined in an ebullating bed reactor under argon at $540^{\circ} \mathrm{C}$ for one hour and in air at $540^{\circ} \mathrm{C}$ for two hours. Chemical analysis indicated $10.6 \%$ by weight iron, and BET surface area measurement indicated $2.9 \mathrm{~m}^{2} \mathrm{~g}^{-1}$. A portion of the calcined product $(46 \mathrm{~g}$.) was impregnated with a binder consisting of $17 \mathrm{~g}$. sodium silicate solution (Fisher Scientific) in $75 \mathrm{ml}$ of water, dried at $125^{\circ} \mathrm{C}$ overnight, ground and sized to $18 / 35 \mathrm{mesh}$, and calcined in a tube furnace exposed to air at $550^{\circ} \mathrm{C}$ for one hour

Chemical analysis indicated that the sample contained $10.11 \% \mathrm{Fe}, 30.23 \% \mathrm{Si}, 0.007 \% \mathrm{SO}_{4}^{2-}$, $0.018 \% \mathrm{Cl}$, and $4.03 \% \mathrm{Na}$, by weight, somewhat higher than the expected percentage of iron after dilution with the binder, which was $9.5 \%$.

Given in Table 1 is a list of the major peaks obtained in the $\mathrm{x}$-ray diffraction pattern of the above calcined sample prior to addition of binder, using $\mathrm{CuK}$ radiation and a solid state detector. The diffraction pattern of hydroxysodalite is given for reference. A small amount of a cancrinite phase may be present in the calcined sample. 


\section{TABLE 1}

\begin{tabular}{|c|c|c|c|}
\hline Calcined Sample & $\begin{array}{l}\text { mple } \\
\text { Relative } \\
\text { Intensity }\end{array}$ & \multicolumn{2}{|c|}{$\begin{array}{c}\begin{array}{c}\text { Hydroxysodalite } \\
\text { (hvdrated, synthetic) } \\
\text { Relative } \\
\text { 2-Theta Intensity }\end{array}\end{array}$} \\
\hline 14.05 & 100 & 34.92 & 100 \\
\hline 24.24 & 84 & 24.54 & 90 \\
\hline 24.46 & 53 & 24.46 & 89 \\
\hline 19.78 & 37 & 24.49 & 88 \\
\hline 34.49 & 17 & 14.08 & 49 \\
\hline 31.47 & 11 & 31.89 & 27 \\
\hline 69.17 & 10 & 43.02 & 23 \\
\hline 11.13 & 10 & 43.08 & 22 \\
\hline 31.79 & 8 & 58.7 & 10 \\
\hline 31.24 & 8 & & \\
\hline 51.88 & 7 & & \\
\hline 11.6 & 7 & & \\
\hline 27.47 & 6 & & \\
\hline 34.65 & 6 & & \\
\hline 20.21 & 5 & & \\
\hline 20.32 & 5 & & \\
\hline 52.39 & 5 & & \\
\hline 40.17 & 5 & & \\
\hline 17.06 & 5 & & \\
\hline 13.42 & 5 & & \\
\hline 61.76 & 4 & & \\
\hline 58.52 & 4 & & \\
\hline 43.12 & 4 & & \\
\hline
\end{tabular}


During the period of this program, we further developed the synthesis of the active iron sodalite [Fe]SOD. We found that the amount of framework iron in the sodalite should be maximized to give high activity. We further determined that the catalytic activity was enhanced by adding additional iron(II) to the exchange sites. The best catalyst, therefore, had iron in both the exchange and the framework positions. The iron sodalite catalyst was characterized by a combination of its elemental analysis, its $\mathrm{x}$-ray powder diffraction pattern, its esr spectrum and some ion exchange studies. We chose manganese as the metal to be inserted into the framework of the second [M]SOD catalyst. As we disclosed in an early Quarterly Report we were unable to prepare [Mn]SOD in a manner analogous to $[\mathrm{Fe}] \mathrm{SOD}$. Neither direct synthetic methods nor post synthesis substitution were effective at rendering a manganese sodalite phase. Since addition of Fe into the exchange sites of framework substituted [Fe]SOD gave rise to the highest methane oxidation activity and methanol selectivities, we added $\mathrm{Mn}$ into the exchange sites of [Fe]SOD. This catalyst, $\mathrm{Mn} n_{x}[\mathrm{Fe}] \mathrm{SOD}$, was tested for methane oxidation activity.

\subsubsection{Metals In Regular Oxidic Matrices}

MnAPO molecular sieves are a member of the AIPO4-based materials patented by Union Carbide (UOP). They are synthesized from gels containing transition metal ions such as $\mathrm{Mn}^{2}$, and it is assumed that the cations are incorporated into tetrahedrally coordinated lattice positions during hydrothermal crystallization of the molecular sieves. There has also been evidence to suggest that some of the manganese cations exist in extraframework sites to balance the negative charge formed by the incorporation of the manganese into the neutral $\mathrm{AlPO}_{4}$ framework.

\subsection{3 $\mathrm{MnAPO}-5.47$}

MnAPO- 5 is a large pore material $(0.8 \mathrm{~nm})$ with a novel structure that is templated by a number of different organics, including diethylethanolamine. MnAPO-5 has excellent thermal and hydrothermal stability. MnAPO-47 has the same topology as the zeolite chabazite and is a small pore material $(0.43 \mathrm{~nm})$; diethylethanolamine in high gel concentrations is the most common templating agent for the 47 structure type. MnAPO-47 is not thermally stable when calcined in air to remove the organic template, and calcination is generally done in an inert atmosphere.

The tested sample was prepared following USP 4,567,029 Ex. 66 . The starting gel ratio was:

2.0 DEEA : $0.167 \mathrm{MnO}: 0.917 \mathrm{Al}_{2} \mathrm{O}_{3}: 1 \mathrm{P}_{2} \mathrm{O}_{5}: 45 \mathrm{H}_{2} \mathrm{O}$

The synthesis was done at $200^{\circ} \mathrm{C}$ for one day, quiescently under autogeneous pressure. The final product was white. Under the microscope, the sample contained two distinct morphologies: cubes and six-sided rods. The six-sided rod morphology is typical of the 5 structure, and the cubes are typical of the 47 structure.

$\mathrm{X}$-ray diffraction pattern of the as-synthesized material was consistent with that of a mixture of 5 and 47 with the predominant phase being 5 (Figure 1). Calcination at $600^{\circ} \mathrm{C}$ in air for three hours yielded a lavender/gray material. The X-ray diffraction pattern of the calcined material showed that 
the 47 structure type had completely collapsed and that the 5 structure type was still present (Figure 2).

The calcined material was sent for chemical analysis and the final product oxide ratio was:

$\begin{array}{cc}\mathrm{Al}_{2} \mathrm{O}_{3} & 0.4 \\ \mathrm{P}_{2} \mathrm{O}_{5} & 0.49 \\ \mathrm{MnO} & 0.11\end{array}$

It can be seen that the divalent manganese substitutes for the trivalent aluminum and not for the phosphorus, and this is consistent with the MeAPO materials and results in a net negative framework charge.

\subsubsection{MnAPQ-11 and FAPO-11}

The eleven structure type in the $\mathrm{AlPO}_{4}$ system is a 10 membered ring material with ellipitical pores. The pores are $3.9 \times 6.3 \mathrm{~A}$ in diameter and are arranged in a one dimensional channel system.

Two framework substituted $\mathrm{APO}_{4}$ 's were pareared during this quarter for testing: FAPO-11 and MnAPO-11.

\subsubsection{FAPO-11}

FAPO-11 was prepared using the following gel composition:

$0.1 \mathrm{Fe}_{2} \mathrm{O}_{3}, 1 \mathrm{Pr}_{2} \mathrm{NH}, 0.9 \mathrm{Al}_{2} \mathrm{O}_{3}, 1 . \mathrm{OP}_{2} \mathrm{O}_{5}, 43 \mathrm{H}_{2} \mathrm{O}$. Digestion was done at $200^{\circ} \mathrm{C}$ for 24 hours. The final product was green, probably indicating incomplete insertion of the iron into the framework. The XRD of the material showed that the material was not fully crystalline and that there were impurity phases present that are probably 5,11 , or 41 .

The sample as calcined at $500^{\circ} \mathrm{C}$ for one hour in air; there was a decrease in crystallinity and the impurity phases were still present. Calcination in nitrogen for two hours gave less of a decrease in crystallinity, but the impurity phases were still present.

A chemical analysis of the calcined material gave an oxide formula of: $\mathrm{Fe}_{0.5}, \mathrm{Al}_{.46}, \mathrm{P}_{49}$. This indicates that the iron is substituting primarily for the aluminum. The bulk analysis was

$\begin{array}{ccc}5.29 & \text { weight \% } & \mathrm{Fe}_{2} \mathrm{O}_{3} \\ 32.87 & & \mathrm{Al}_{2} \mathrm{O}_{3} \\ 49.03 & & \mathrm{P}_{2} \mathrm{O}_{5}\end{array}$

Preparation of this material should be repeated to produce a more crystalline material with better iron insertion. 


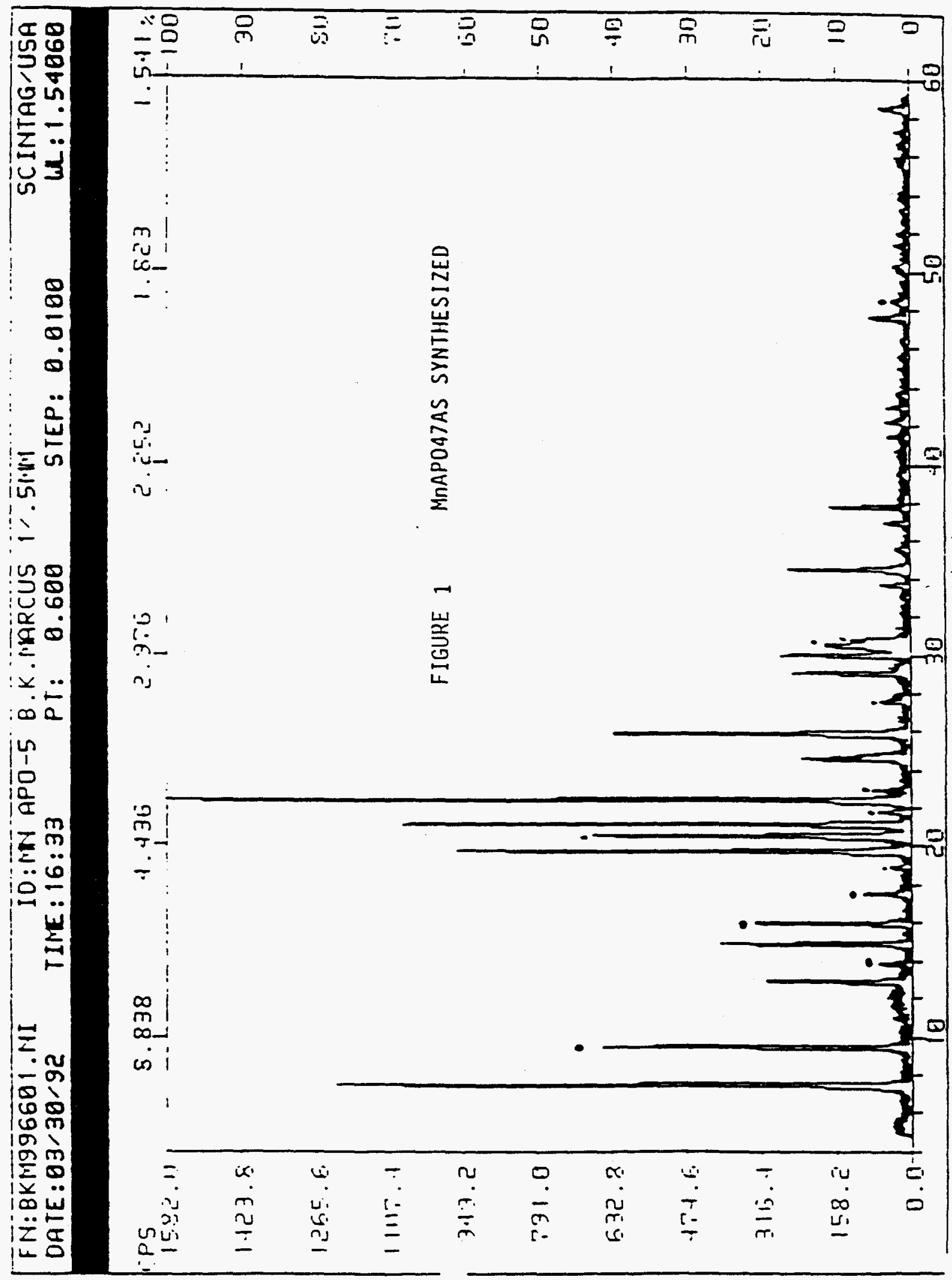




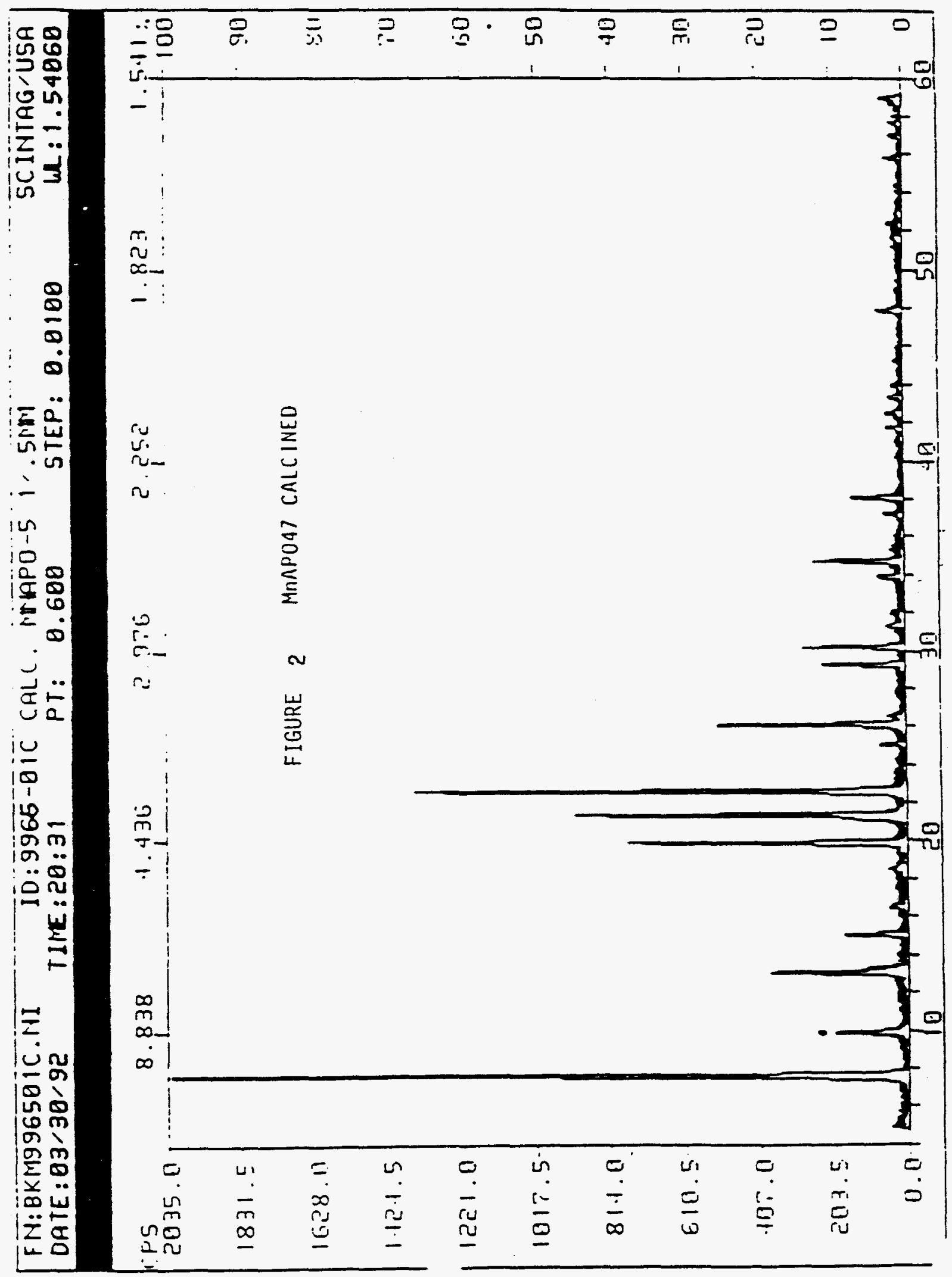




\subsection{6 $\mathrm{MnAPO}-11$}

MnAPO-11 was prepared from a gel with the composition: $0.167 \mathrm{MnO}, 1$ Diisopropylamine, $0.917 \mathrm{Al}_{2} \mathrm{O}_{3}, 1 \mathrm{P}_{2} \mathrm{O}_{5}, 40 \mathrm{H}_{2} \mathrm{O}$.

The gel was digested at $200^{\circ} \mathrm{C}$ for 3 days. The XRD was consistent with a well-crystallized 11 sample. Calcination at $500^{\circ} \mathrm{C}$ for one hour in air showed some decrease in crystallinity; calcination for 4 hours in nitrogen gave excellent structure retention.

Chemical analysis of the material gave a framework oxide formula of:[ $\left.\mathrm{Al}_{.42} \mathrm{P}_{55} \mathrm{Mn}_{.03}\right]$. In this case, the amount of phosphorus is high and could indicate an error in the analysis.

The five structure type in the $\mathrm{AlPO}_{4}$ system is a 12 ring material with a circular pore that is 7.3 $A$ in diameter. The material is less dense than the 11 'structure type, but it also has a one dimensional pore system.

\subsection{CATALYST TESTING}

An active surface containing metal oxo centers might generate alkyl radicals according to equation (6) (15):

$$
\mathrm{MO}+\mathrm{RH} \rightarrow \mathrm{R} \bullet+\mathrm{MOH}
$$

We have synthesized a variety of all-inorganic phases which we thought capable of generating high oxidation state iron oxo species under appropriate conditions and examined them for catalytic activity. Examples are framework substituted iron sodalite $(16,17)$ and amorphous silicoferrates, containing isolated iron atoms or oxo-bridged multi-nuclear iron centers respectively in varying amounts in predominantly siliceous frameworks.

\subsubsection{Testing of Iron Sodalite}

Early experiments were conducted in barricaded stainless steel packed bed reactors under steady-state conditions using 3:1:2.5 methane:air:water fed continuously over the catalysts at 918 psig. Although methanol selectivities were in general quite low in this reactor $(<30 \%)$ probably due to reactor induced methanol decomposition $(13,18)$, differences among catalysts were noted. Silica supported bulk iron oxide demonstrated extremely low selectivity to methanol, forming mostly $\mathrm{CO}_{2}$. In contrast, sodium exchanged iron sodalite containing $13 \%$ iron, Figures 5,6 gave higher methanol selectivity than either its aluminum analog, hydroxysodalite, or than a lower iron content iron-substituted pentasil of the ZSM-5 structure when each was bound with sodium silicate then calcined in air. Pretreatment of the iron sodalite catalyst by sodium azide decomposition to remove traces of acidity (19) resulted in even higher methanol selectivities; whereas, mild acid exchange resulted in virtually no methanol production. An amorphous iron silicate prepared similarly to the precursor gels for iron sodalite but calcined instead of being allowed to crystallize to the zeolite was found to be less active. Iron (ll) ion exchange of the iron sodalite resulted in improved performance. 


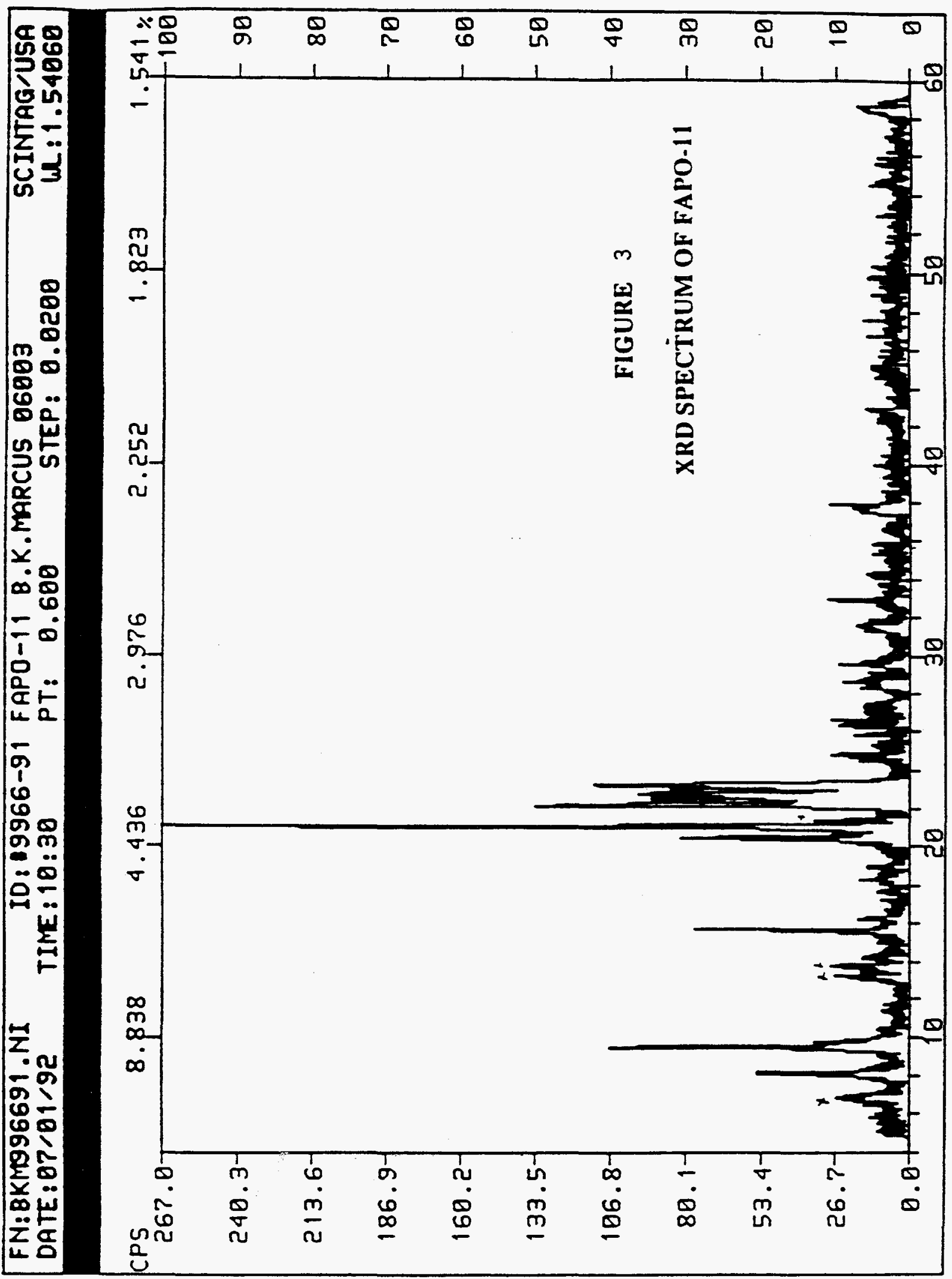




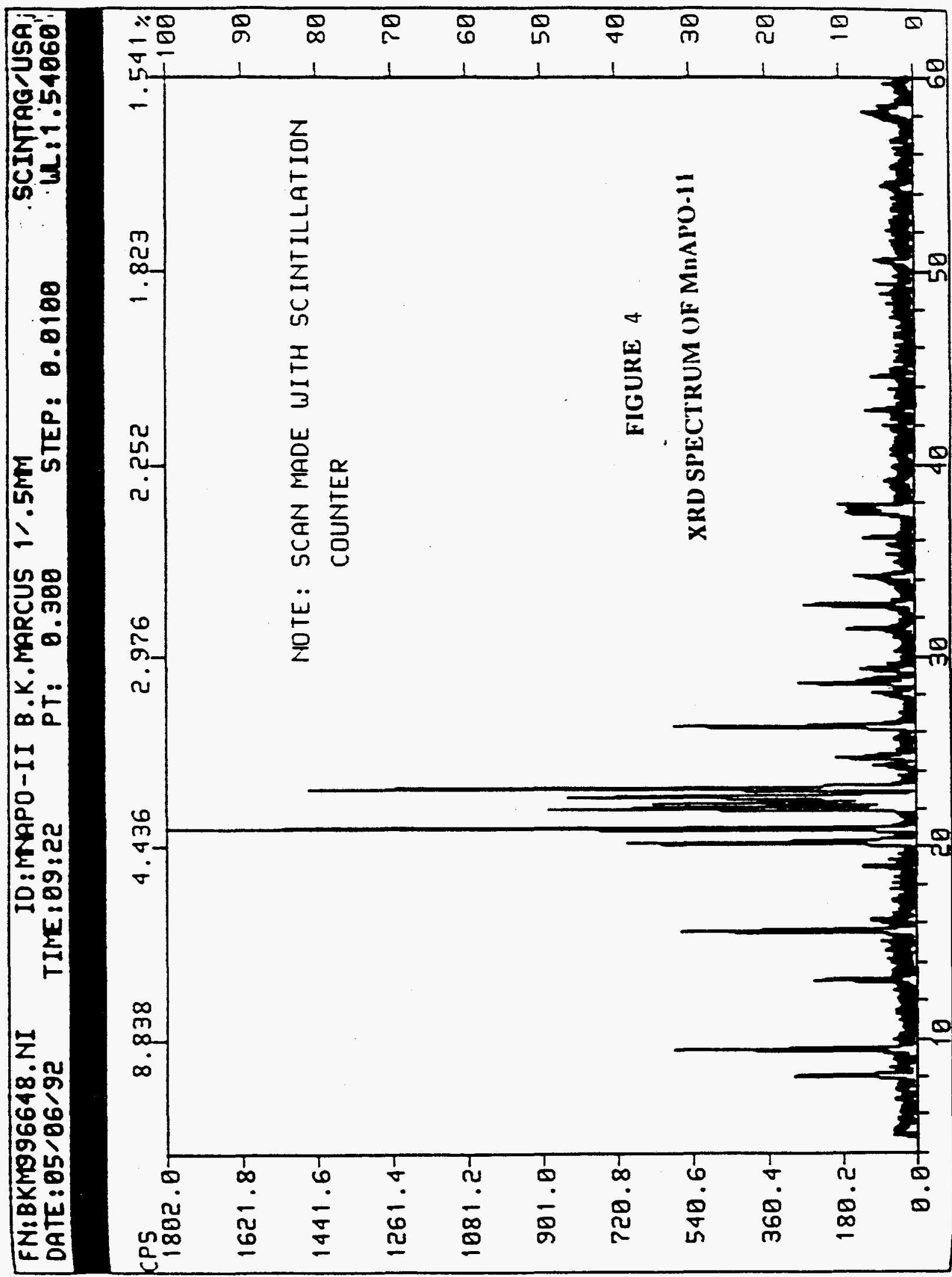




\section{LIGHT ALKANES TO LIQUID OCTANE}

METHANE TO METHANOL

$\mathrm{CH}_{4}+1 / 2 \mathrm{O}_{2} \longrightarrow \mathrm{CH}_{3} \mathrm{OH}$

METHANE (AIR) METHANOL

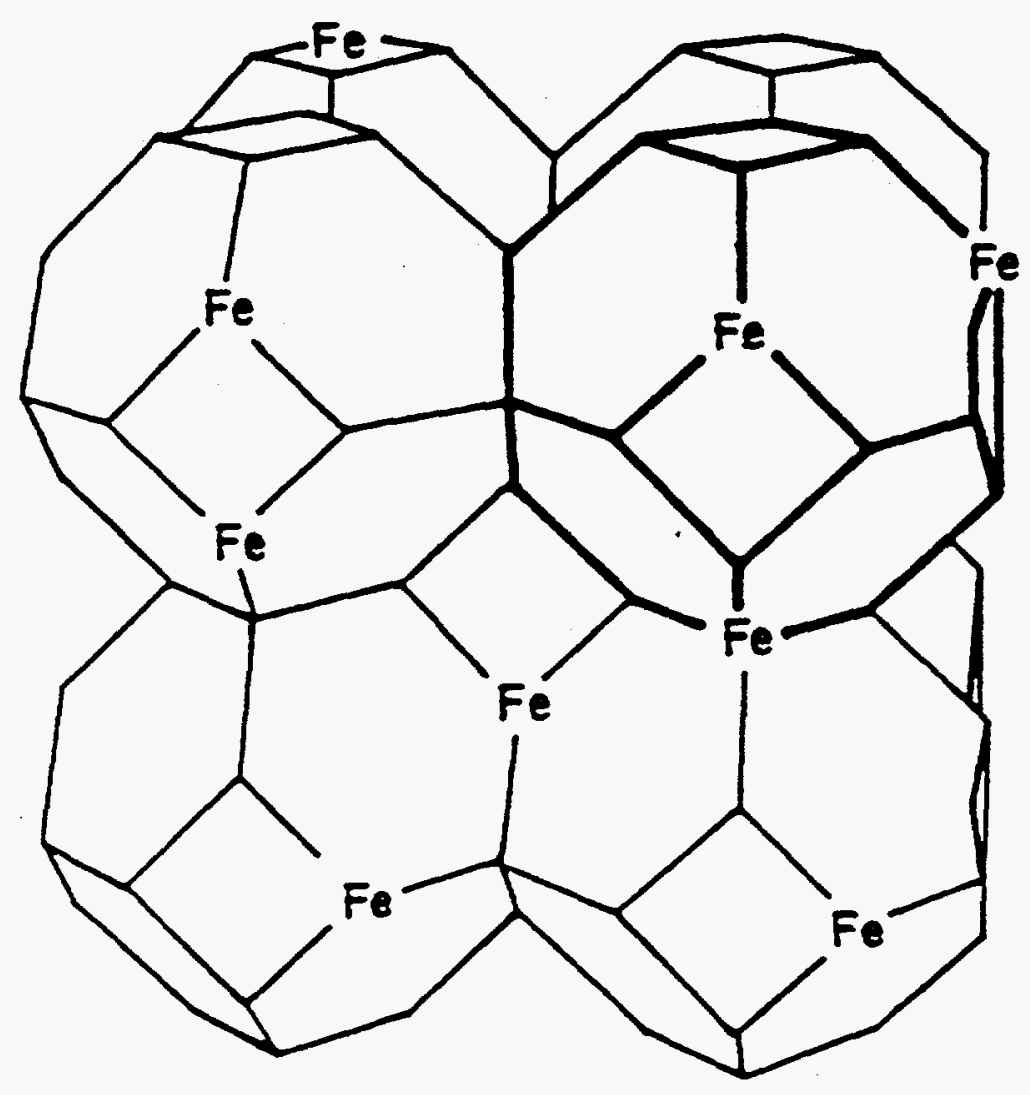

CATALYST $=15 \%$ Fe IN SODALITE CONDITIONS: $T=420^{\circ} \mathrm{C}, \mathrm{G}$ HSV 600 ,

3/, METHANE/AIR

RESULTS GOAL

$\mathrm{O}_{2}$ CONVERSION

METHANE CONVERSION

METHANOL SELECTIVITY

$\begin{array}{rl}90 \% & 90 \% \\ 7 \% & 20 \% \\ 70 \% & 80 \%\end{array}$




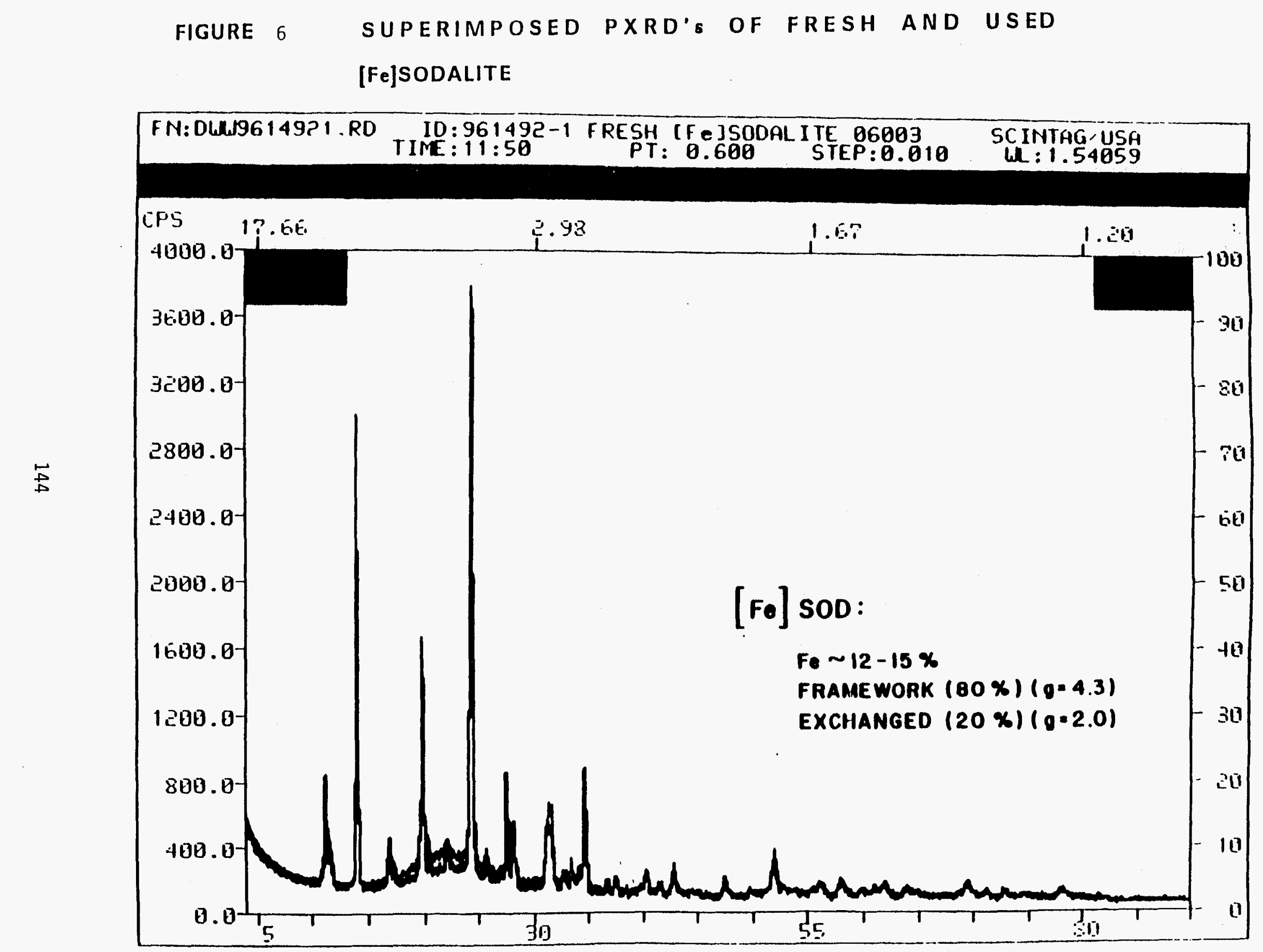


During the course of this work it became apparent that methanol decomposition induced by the stainless steel reactors could result in low methanol yields. In addition, we found that significant backmixing resulted in diffusion of much of the methanol formed at the top of the catalyst bed into the vapor space above the reactor when operating at low flow rates. For this reason we constructed a quartz lined packed bed reactor in which a portion of the gas flow at the head of the reactor bed could be drawn off instead of passing through the entire packed bed. The amount of product drawn from above the bed was controlled by a needle valve and could be either cold trapped or recombined with the main flow exiting the bottom of the catalyst bed prior to a liquid condenser.

Results using this quartz lined reactor at 3:1 methane:air without water cofeed through the bed are listed in Tables 2 and 3, Figure 7. In contrast to measurements in the metal reactor, our methanol selectivities in the quartz reactor with the by-pass valve open improved to about $70 \%$ selectivity at ca. $7 \%$ methane conversion over silicate-bound iron sodalite after an aging period. Closing of the needle valve such that the entire vapor stream passed through the bed resulted in much lower methanol selectivity and correspondingly lower conversion (Table 3). Comparison of the performance of silicate-bound iron sodalite with glass beads and with silicate-bound hydroxysodalite with the by-pass valve open is shown in Table 3 . In all cases, the use of [Fe]SOD resulted in enhanced methanol yield and selectivities relative to control runs. By applying our kinetic model and assuming only an increase in $\mathrm{CH}_{3} \bullet$ and no methanol decomposition, we estimate a methyl radical production rate enhancement of at least an order of magnitude from [Fe]SOD relative to glass beads in these experiments.

Any scheme which depicts a possible reaction pathway over silicoferrate catalysts must account for the following observations: a) a high iron concentration is required; b) supported and bulk iron oxides are poor catalysts; c) the activities of amorphous, high-iron catalysts are inferior to that of crystalline $\mathrm{Fe}[\mathrm{SOD}]$; d) the performance of $\mathrm{Fe}[\mathrm{SOD}]$ improves with $\mathrm{Fe}(\mathrm{ll})$ exchanged; e) an induction period during which some deferration is likely to occur is observed, and f) acidity is detrimental, but basicity is beneficial. A suggested catalytic reaction pathway which would account for these observations implicates a binuclear iron site to activate and split the oxygen molecule, Figure 8. Although several variants of this cycle can be drawn, a key feature is the proximity of an extra framework hydroxyiron cationic species to a framework iron site at which ion exchange capacity develops in the structure. The two irons might act in concert to activate oxygen at elevated temperatures. Formation of binuclear oxo-bridged Fe(ll1), could represent a deactivation path in this scheme, and this could account for the poor performance of iron oxide phases in our reactors.

\subsubsection{Effects of Reactor Design on Methane Oxidation}

In past Quarterly Reports we have discussed the question of reactor design and how it influences reaction rate and product selectivity. We have seen that a fully loaded packed bed reactor did not give the high selectivities that were seen in a reactor which contained a large empty void volume ahead of the heated packed bed of catalyst to which was attached a by-pass line leading to the reactor exit stream (4). This reactor had features which combined: 1) a lower temperature reaction zone in which a radical flux generated by the hot catalyst could selectively produce partial oxidation products, 2) a reactive distillation to remove methanol as formed, and 3) a component of the reaction mixture which passed through the catalyst bed in a conventional way. Using this reactor, we found 


\begin{tabular}{|c|c|c|c|c|c|}
\hline Catalyst & $\begin{array}{c}\text { GHSV } \\
h^{-1}\end{array}$ & $\begin{array}{l}\text { Bed T } \\
\cdot{ }^{\circ} \mathrm{C}\end{array}$ & $\begin{array}{l}\mathrm{CH}_{3} \mathrm{OH} \\
\text { Sel., } \%\end{array}$ & $\begin{array}{l}\mathrm{CH}_{4} \\
\text { Conv., } \%\end{array}$ & $\begin{array}{l}\mathrm{O}_{2} \\
\text { Conv., \% }\end{array}$ \\
\hline \multirow[t]{6}{*}[\mathrm{Fe}]{$\mathrm{SOD}$} & 530 & 407 & 64 & 4.6 & 76 \\
\hline & & 461 & 70 & 5.7 & 90 \\
\hline & & 418 & 68 & 5.4 & 91 \\
\hline & & 422 & 65 & 5.4 & 92 \\
\hline & & 432 & 63 & 5.5 & 90 \\
\hline & & 442 & 64 & 6.1 & 90 \\
\hline \multirow[t]{3}{*}{ Hydroxysodalite } & 530 & 404 & - & 0.0 & 0 \\
\hline & & 430 & 9 & 0.3 & 4 \\
\hline & & 445 & 24 & 5.1 & 84 \\
\hline \multirow[t]{5}{*}{$\mathrm{Fe}_{x} \mathrm{O}_{y} / \mathrm{SiO}_{2}(10-15 \%)$} & 530 & 431 & 26 & 5.1 & 85 \\
\hline & 700 & 398 & 10 & 0.5 & 11 \\
\hline & & 409 & 18 & 1.2 & 16 \\
\hline & & 420 & 20 & 2.7 & 74 \\
\hline & & 428 & 23 & 3.9 & 83 \\
\hline \multirow[t]{2}{*}[\mathrm{Fe}]{$\mathrm{ZEOL}$} & 700 & 400 & 33 & 2.4 & 52 \\
\hline & & 430 & 34 & 3.8 & 74 \\
\hline
\end{tabular}

a $P=800$ psig, 3:1 methane:air. Quartz lined reactor with by-pass open. 

TABLE 3 Effect of By-Page In Methane Oxidation Over Fe[SOD] in Quartz Lined
Tubular Reactor

\begin{tabular}{cccccccc}
\hline By-Pase Status & Production & Rate & mmol/h & $\mathrm{O}_{2}$ Consum & $\mathrm{CH}_{3} \mathrm{OH}$ & $\mathrm{CH}_{4}$ \\
& $\mathrm{CH}_{3} \mathrm{OH}$ & $\mathrm{CO}$ & $\mathrm{CO}_{2}$ & mmoles & Selc. $\%$ & Conv. $\%$
\end{tabular}

$\begin{array}{lllllll}\text { Closed } & 1.6 & 1.6 & 0.4 & 3.8 & 44 & 4.8 \\ \text { Open, no trap } & 3.5 & 0.6 & 0.7 & 4.0 & 72 & 6.5 \\ \text { Open, probe } 15 \mathrm{~cm}^{\mathrm{b}} & 2.04 \text { bed } & 1.0 & 0.5 & 4.3 & 71 & 6.0 \\ & 1.60 \mathrm{trap} & & & & & \\ & 3.64 \text { total } & & & & & \\ & & & & & & \\ & & & & & \\ & & 1.1 & 0.7 & 5.0 & \end{array}$

a GHSV $=550 \pm 30 \mathrm{~h}^{-1}, \mathrm{P}=800 \mathrm{psig}, \mathrm{T}$ bed $=427 \pm 2^{\circ} \mathrm{C}, 3: 1$ methane:air feed.

b Distance above bed of by-pass probe tip. 
FIGURE 7

TEMPERATURE AND SPACE VELOCITY DEPENDENCE OF METHANOL SELECTIVITY IN QUARTZ BYPASS REACTOF

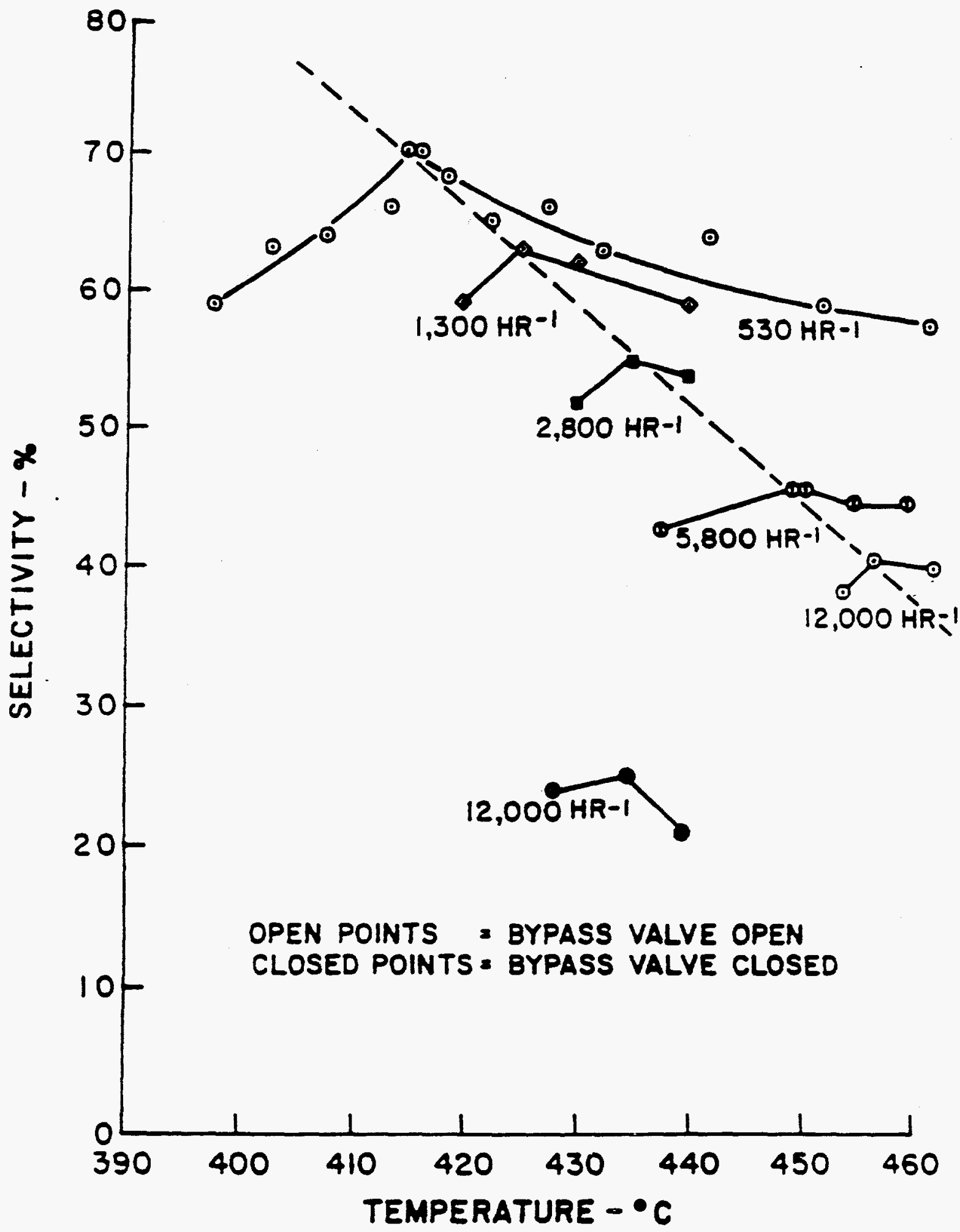




\section{FIGURE 8}

\section{HYPOTHETICAL CATALYTIC CYCLE FOR SILICOFERRATE ACTIVITY}

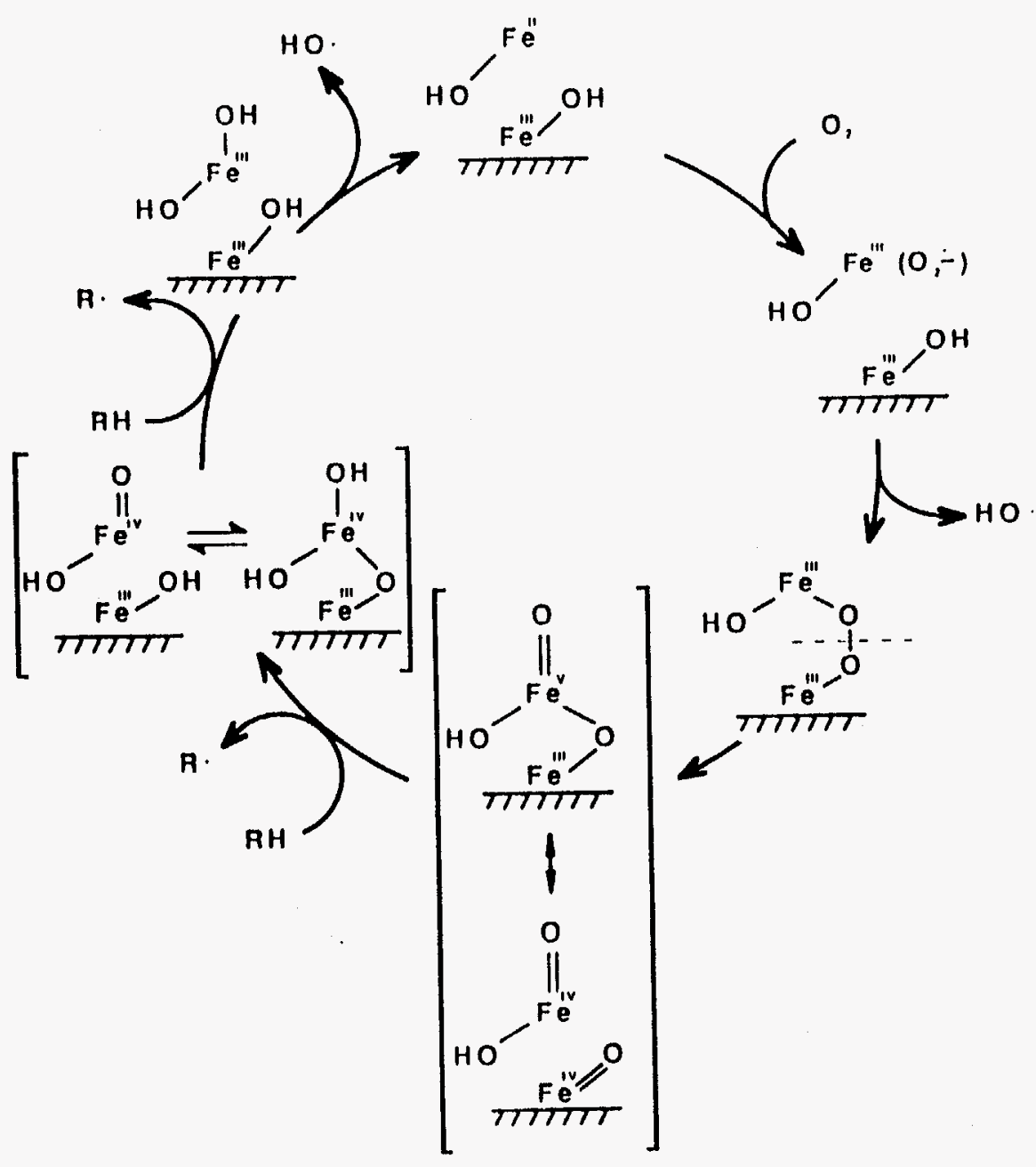


that selectivities for the iron sodalite-catalyzed oxidation of methane to methanol which did not reach $50 \%$ in a conventional packed bed reactor, exceeded $70 \%$.

We have compared three different reactor designs for the catalytic oxidation of methane to methanol. A complex design having a combination of a low-temperature back-mixed zone over a hot catalyst coupled with reactive distillation is shown in Fig. 9. Results of methane oxidations in this reactor are given in Table 3, and Fig. 8. Though relatively high selectivity (70\%) to methanol can be achieved in this reactor system, it is too complex to use to generate unambiguous catalyst screening data.

We have constructed two simpler systems which separate the catalytic activity from the reactive distillation aspects. One system operates as simple downflow reactor in the plug flow mode, Fig. 10. In this system under conditions of temperature and pressure similar to those used in the complex reactor, but at considerably faster flow rate, iron sodalite catalyzes the oxidation of methane to methanol in $50 \%$ selectivity.

Another system which operates in the back-mixed mode, Fig. 11, gives methanol in about $30 \%$ selectivity at similar temperature, pressure and flow rate. Clearly, reactor design is critical in conducting these oxidations and has been closely examined. We have screened reactions in the simple plug-flow and back-mixed reactors but have considered beneficial effects of more complex design when appropriate to achieve more optimal results.

In the by-pass reactor we were at least partially optimizing several very important features of this reaction. The complexity of the system, however, obscured the origins or the extent of contribution of the several effects that were simultaneously at work. We realized that we needed to disentangle these effects and examine them systematically. There are at least four desirable goals that our reactor should help us to meet: 1) it should allow us to achieve high rates and selectivity, 2) it should be straightforward enough to have ultimate utility in scaling up a catalytic process, 3 ) it should allow us to screen catalysts in the most effective way possible, and 4) it should allow us to understand and control the factors leading to high selectivity. It was clear that while the by-pass reactor was doing very well regarding the first feature, it was unsatisfactory with regard to the other three.

For these reasons we constructed and operated two additional reactor types over the past several months. One reactor was a gold-plated 50cc Autoclave Engineers CSTR with Robinson-Mahoney Internals. This is a back-mix reactor which enables us to control and understand temperature effects and to independently vary residence time and degree of back-mixing. We have screened both a silica surface and our iron sodalite catalyst at $400^{\circ} \mathrm{C}$ under conditions in which catalytic reactions and non-catalytic gas phase oxidation compete. We were able to show far higher rates and selectivities in the presence of the iron catalyst. This enables us to assess the contribution of catalytic generation of methanol to the process at these temperatures. We have used this reactor to generate the data which enables us to gauge the differences in catalysts apart from the other effects present in the by-pass reactor. 


\section{QUARTZ LINED BYPASS REACTOR SYSTEM}

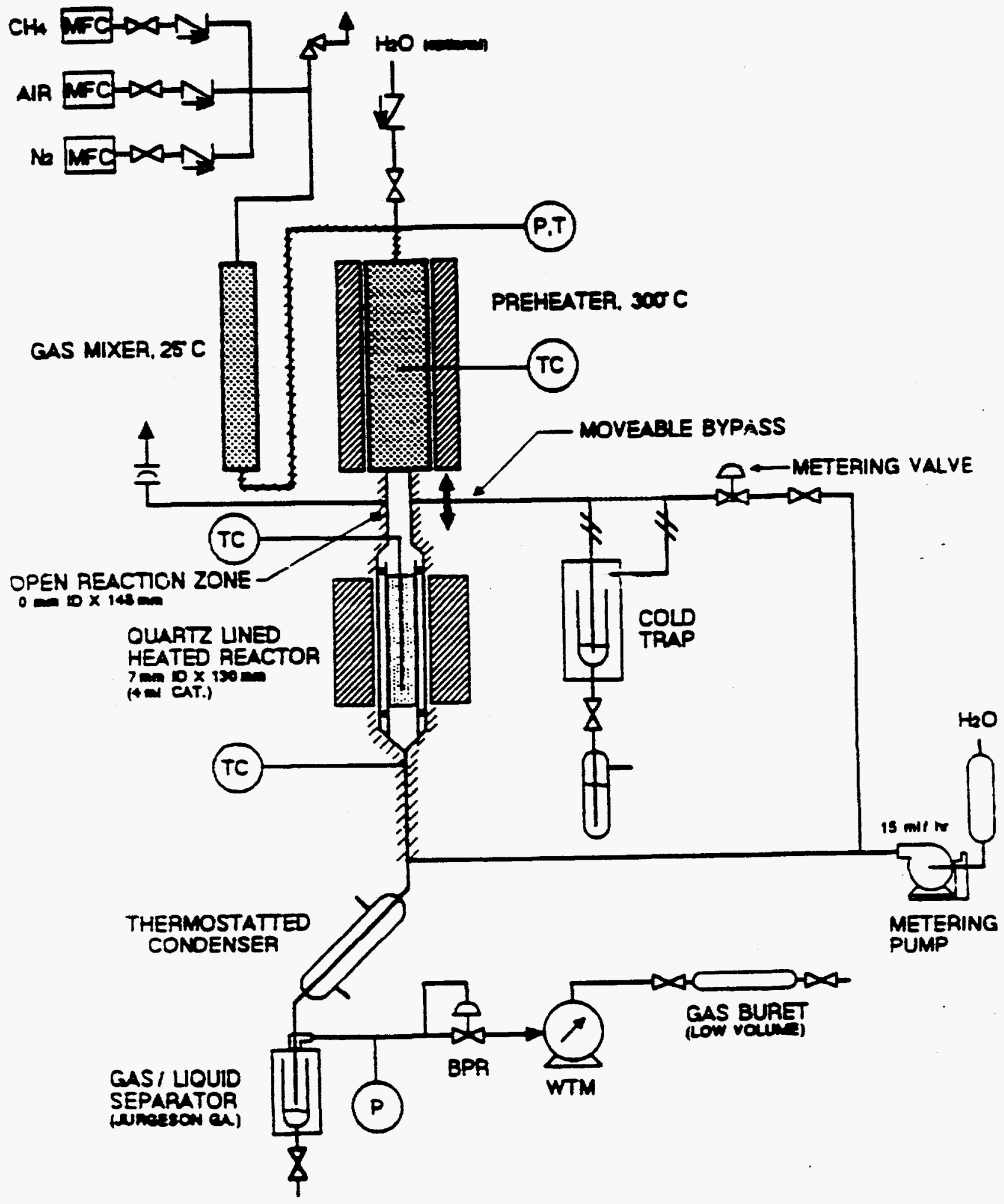




\section{PLUG FLOW OPERATION}

FIGURंE 10

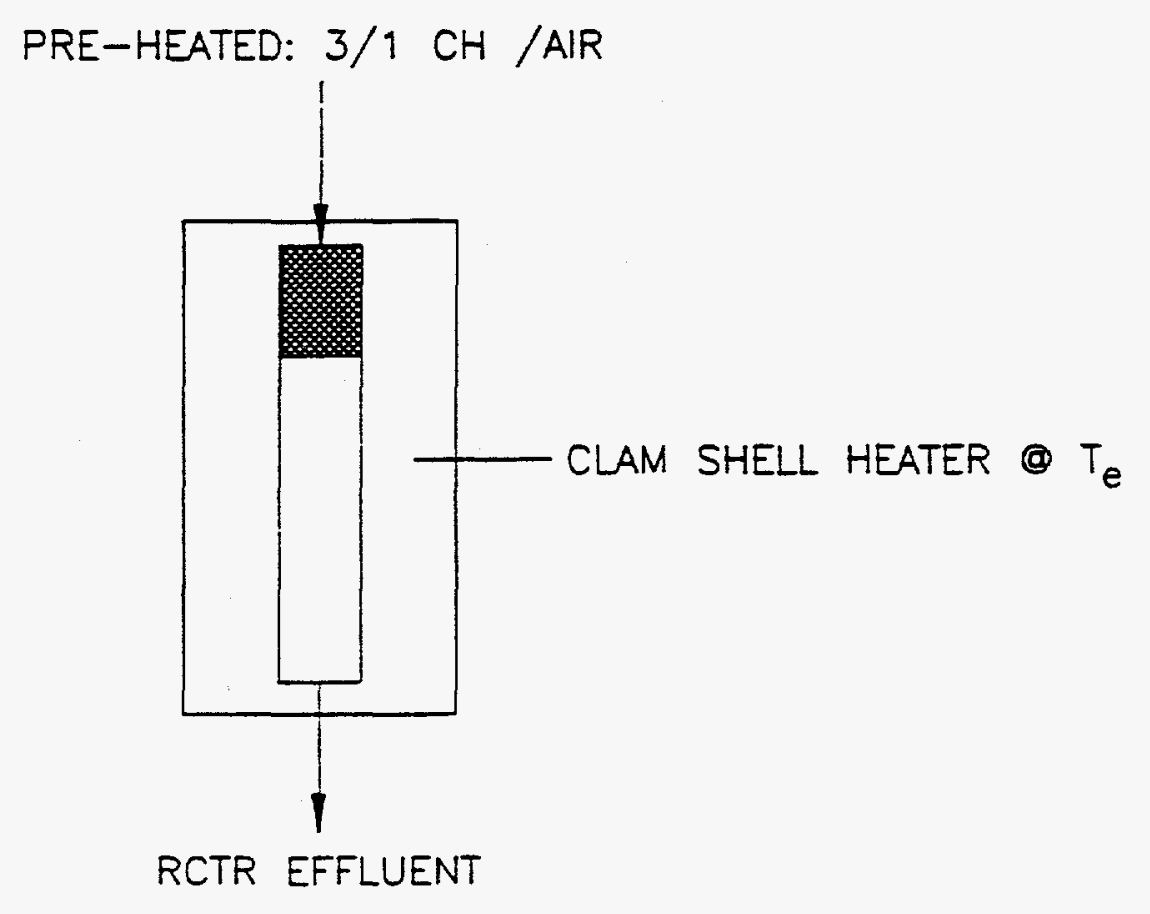

CATALYST $=\mathrm{Fe}_{X}[\mathrm{Fe}] \mathrm{SOD}$

$\underline{T_{e}} \mathrm{~T}_{\mathrm{i}}$ GHSV SEL,\% $\underline{\mathrm{O}_{2} \text { CONV.,\% }} \mathrm{CH}_{4}$ CONV.

$\begin{array}{llllll}405 & 404 & 2,800 & 45 & 99 & 6\end{array}$

$\begin{array}{llllll}410 & 406 & 5,800 & 50 & 90 & 6\end{array}$

$\begin{array}{llllll}416 & 409 & 12,000 & 48 & 48 & 3\end{array}$ 


\section{CSTR WITH ROBINSON-MAHONEY INTERNALS AND EXTERNAL BYPASS}

FIGURE 11.

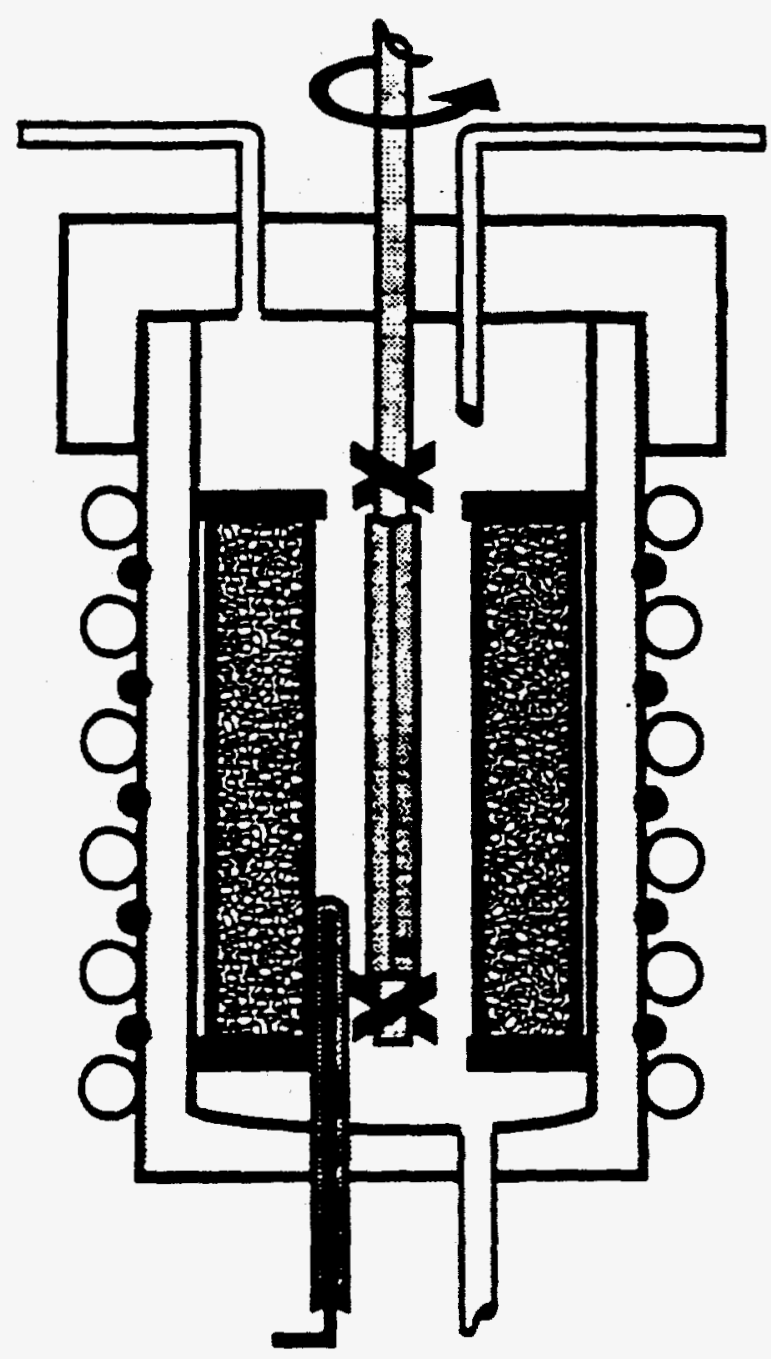

CATALYST

$\mathrm{Fe}_{x}[\mathrm{Fe}] \mathrm{SOD}$

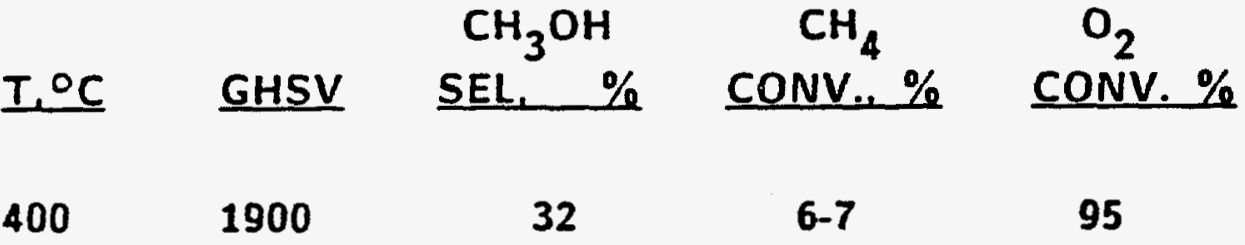


Simultaneously we were interested in studying the effect of a cool quench zone for radicals which exit a hot catalytic surface during oxidation of methane. This may be one of the phenomena that elevated the methanol yields in the by-pass reactor. While we searched for the best design to study this phenomenon, we used the expedient of partial packing of a tubular reactor to allow a short, hot catalyst zone to contact the reacting gases followed by a longer void space whose temperature can be controlled. We have completed the study of several catalysts. Even when no attempt is made to cool the void zone, methanol selectivity in this reactor configuration is much higher than in a fully packed catalyst bed indicating that perhaps product formation continues in the void zone without methanol decomposition.

\subsubsection{Catalytic testing in a Downflow Reactor}

We discussed early observations on the effect of reactor design on methanol productivity in the vapor phase air-oxidation of methane. We have found that reactions conducted under similar conditions of temperature and pressure at methane conversions between 4 and $8 \%$, a) gave up to $30 \%$ methanol selectivity in a well-controlled back-mixed reactor, b) gave up to $50 \%$ selectivity in plug flow operation depending on the reactor/catalyst bed configuration, and c) gave greater than $70 \%$ selectivity in partially plug flow operation wherein methane was activated hot and reacted at a cooler temperature in a zone from which reactive distillation could occur. Figure 12 indicates results of methane oxidation in a fully or partially packed reactor operating near plug flow conditions.

It can be seen that practically no methanol is produced in a tube fully packed with glass beads at $390^{\circ} \mathrm{C}$, whereas methane can be produced in over $30 \%$ selectivity when a catalyst, $\mathrm{Fe}_{\mathrm{x}}[\mathrm{Fe}] \mathrm{SOD}$, was used An even more effective mode of operation was established when only the top third of the reactor was packed with catalyst and the bottom $2 / 3$ was left empty. Even though the conversion was less, the selectivity to methanol was $50 \%$. The interpretation of this result that radicals were generated on the hot catalyst surface which migrated to the vapor space to react. If one filled this vapor space with a relatively inert material (glass beads), reaction of these radicals was quenched and little product was generated. Similarly, if the methyl radicals generated on the catalyst were sent rapidly through a capillary they did not propagate chains and low product yields resulted.

These results are consistent with a reaction pathway in which methyl radicals are generated at the top of a catalyst bed. Methanol and other oxidation products are formed in the vapor space. If the entire reactor space is filled with catalyst, a significant amount of methanol decomposition occurs. Methanol decomposition over Fex[Fe]SOD has been demonstrated with and without air present. If the majority of the reactor space is empty, a gas phase methane oxidation ensues in the presence of a high methyl radical flux. We have shown by a kinetic model of gas phase methane oxidation at this temperature that increased methyl radical flux increases both reaction rate and methanol selectivity. If the methyl radicals are quenched after exiting the radical generating zone, low product yields result. These results therefore are consistent with a catalyst which operates by producing methyl radicals which generate methanol in subsequent gas phase radical pathways. 


\section{METHANE OXIDATIONS}

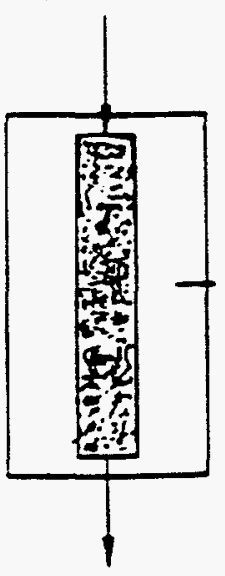

A

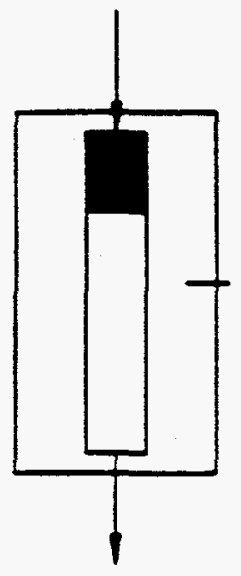

B

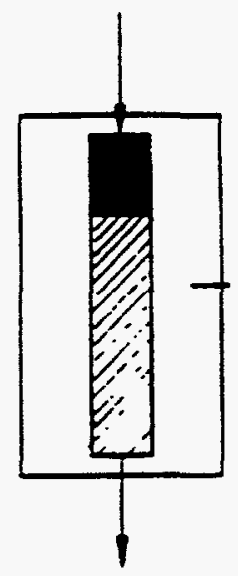

C
PRE-HEATED: $3 / 1 \mathrm{CH}_{4} / \mathrm{NR}$

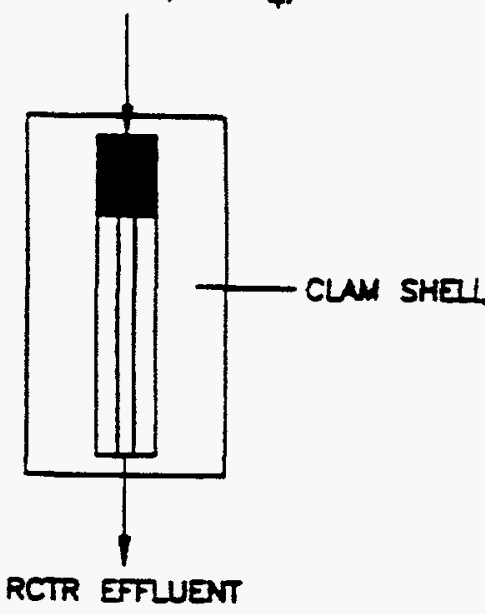

D
PACKING

CONFIG, CATALYST

A $F e_{x}[\mathrm{Fe}] \mathrm{SOD}$

A Glass Beads

B

B

$c$

D

$\mathrm{Fe}_{x}[\mathrm{Fe}] \mathrm{SOD}$

10 Glass Beads (2/3)
5.9

32

METHANOL

1.9
99

10

0.2

10

0.02

67

10

8

0.2

4.0

50

2.0

0.9

34

0.3

45

0.1

$\mathrm{T}=390^{\circ} \mathrm{C}, \mathrm{P}=800 \mathrm{psig}, 3 / 1 \mathrm{CH}_{4} / \mathrm{AlR}, \mathrm{GHSV}=2800 \mathrm{hr}^{-1}$ 
Finally, as discussed in past Quarterly Reports, our work on kinetic modelling of the gas phase oxidation of methane via the "heterogeneous-homogeneous" mechanism suggests that it will be difficult to achieve greater than $80 \%$ methanol selectivity at greater than $20 \%$ conversion by this mechanism. Our analysis indicates that greater than $80 \%$ methanol selectivity at greater than $20 \%$ methane conversion will be needed for such a process to be economic. This has encouraged us to begin a new catalyst synthesis initiative to produce catalytic materials which encourage "rebound" of methyl radicals to $\mathrm{OH}$ groups on the catalyst as a surface mechanism for methanol formation. These sites should exist in a very hydrophobic environment so that methanol is rapidly expelled as formed - otherwise oxidative decomposition will lower selectivity and yield.

\subsubsection{Oxidation of Ethane Over Fex[Fe]SOD}

We have also oxidized ethane over the iron exchanged and framework substituted sodalite that we have used for the catalytic oxidation of methane. Table 4 gives the product analysis for several oxidation reactions. We have obtained good material balances for these reactions and are confident of the reliability of the data. The vapor phase non-catalytic oxidation of ethane has been has been reported recently by $\mathrm{H}$. Gesser, et al., Energy and Fuels, 5, 1991, 423-427. These authors did not report the presence of a number of important products including hydrogen,formaldehyde, ethylene, acetone, propane and butane, among others. They also reported slightly higher alcohol selectivities than we observed. Of particular importance is the formation of formaldehyde which is difficult to analyze by glpc. We have begun to use differential pulse polarography with excellent success to get quantitative analysis of formaldehyde in the aqueous reactor effluent (see below).

It is important to detect significant quantities of formaldehyde in the vapor phase oxidation of ethane since it seems likely that it is a key product in the essential step for forming methanol and perhaps other $C_{1}$ products, eq. 1 ., below:

$$
\begin{aligned}
& \mathrm{C}_{2} \mathrm{H}_{5} \mathrm{O} \longrightarrow \mathrm{CH}_{2} \mathrm{O}+\mathrm{CH}_{3} \bullet+\mathrm{CH}_{3} \mathrm{OH} \\
& \mathrm{C}_{2} \mathrm{H}_{5} \mathrm{O}+\mathrm{C}_{2} \mathrm{H}_{6} \longrightarrow \mathrm{C}_{2} \mathrm{H}_{5} \mathrm{OH}+\mathrm{C}_{2} \mathrm{H}_{5} \bullet
\end{aligned}
$$

As Gesser points out, the ratio of the rate constants for reactions 1 and 2 may play a key role in the ratio of methanol to ethanol or in the $C_{1} / C_{2}$ ratios in the oxidations of ethane. 
TABLE 4

\section{OXIDATIONS OF ETHANE OVER $\mathrm{Fe}_{x}[\mathrm{Fe}] \mathrm{SOD}^{a}$}

\begin{tabular}{|c|c|c|c|c|c|c|c|c|}
\hline $\begin{array}{l}\text { Gas Flow } \\
\text { Mle/Min }\end{array}$ & $I^{\circ} \mathrm{C}$ & $\overline{\mathrm{CH}_{3} \mathrm{OH}}$ & $\frac{\text { Carbon Se }}{\mathrm{C}_{2} \mathrm{H}_{5} \mathrm{OH}}$ & $\frac{\text { ectivity }}{\mathrm{CH}_{2} \mathrm{O}}$ & $\mathrm{CH}_{3} \mathrm{CHO}$ & $\begin{array}{l}\text { Ethane } \\
\text { Conv. \% }\end{array}$ & $\begin{array}{l}\text { Alcohol } \\
\text { Sel. \% }\end{array}$ & $\begin{array}{l}\text { Liq Oxys. } \\
\text { Sel. \% }\end{array}$ \\
\hline 30 & 284 & 28.1 & 21.8 & 9.1 & 5.1 & 4.6 & 49.9 & 65.1 \\
\hline 45 & 305 & 26.9 & 17.8 & 6.8 & 4.5 & 5.3 & 44.7 & 56.0 \\
\hline 188 & 310 & 25.7 & 12.3 & 7.3 & 2.6 & 3.6 & 38.0 & 47.9 \\
\hline 286 & 313 & 26.1 & 7.8 & 7.4 & 3.9 & 4.0 & 33.9 & 45.2 \\
\hline
\end{tabular}

a $P=800$ psig $3 / 1 C_{2} \mathrm{H}_{5} /$ Air 


\subsubsection{Formaldehyde Analysis}

Formaldehyde analysis in the presence of methanol was conducted on diluted aliquots of the aqueous reaction products from ethane oxidations by the differential pulse polarography technique. Direct analysis at a DME using $1 \mathrm{sec}$. drop time, $2 \mathrm{mv} / \mathrm{sec}$ scan rate, $50 \mathrm{mv}$ pulse height, in deaerated $0.1 \mathrm{M}$ lithium hydroxide/ $0.1 \mathrm{M}$ lithium chloride electrolyte resulted in a diagnostic wave for formaldehyde at $-1.65 \mathrm{~V}$ vs. SCE reference. Analysis of standards prepared by serial dilution of a prestandardized stock solution of formaldehyde resulted in linear calibration plots showing negligible residual current in the absence of analyte and exhibiting a typical response of $50 \mathrm{nA}$ peak height per each ppm concentration. Stock solutions were standardized by oxidation of formaldehyde to formic acid with hydrogen peroxide followed by repetitive titrations with standard base to a bromthymol blue end point. The technique is capable of high precision down to $\mathrm{ppb}$ concentrations.

The aqueous reactor effluent from four ethane oxidations was analyzed by this technique and the results are shown in Figures 13-16.

\subsubsection{Oxidation of Methane Over MnAPO-X}

We have been able for the first time to prepare an active and selective methane oxidation catalyst having manganese in the framework of a zeolitic material. In past reports we have disclosed that framework-substituted iron sodalite can be an active and relatively selective catalyst converting methane to methanol. All attempts to incorporate large amounts of manganese into the sodalite framework failed. Supported manganese catalysts such as manganese oxides on silica or alumina largely gave combustion to carbon oxides with little or no methanol formed. We have now found a new and as yet uncharacterized crystalline aluminophosphate phase containing over $8 \%$ manganese which catalyzes selective oxidation of methane and produces significant amounts of methanol below $400^{\circ} \mathrm{C}$. Table 5 compares the activity of alumina (iron and trace metals not removed), silica, Fex[Fe]Sod, and MnAPO-X under comparable conditions.

\subsubsection{Oxidation of Ethane and Methane Over Framework Substituted Zeolites}

Table 6 and 7 show the results of the catalytic oxidation of ethane and methane over a series of three framework substituted zeolites: Fe FeSOD,MnAPO-5/47, and CoAPSO-5/47. It can be seen that the two metal substituted aluminophosphates have greater low temperature activity than does our iron sodalite catalyst. Reactions were run in a top-loaded downflow reactor of a design not intended to maximize methanol selectivity. Nonetheless, the important feature of this work is that the $\mathrm{C}-\mathrm{H}$ bond of methane is more readily broken using metal substituted aluminophosphate catalysts than with iron sodalite - the most active catalyst previously found in our laboratories. Methane can be activated at temperature $30^{\circ} \mathrm{C}$ or more lower using the aluminophosphate catalysts than with iron sodalite. Low temperature activation of methane is crucial to the development of new catalysts capable of surface activation of methane. 


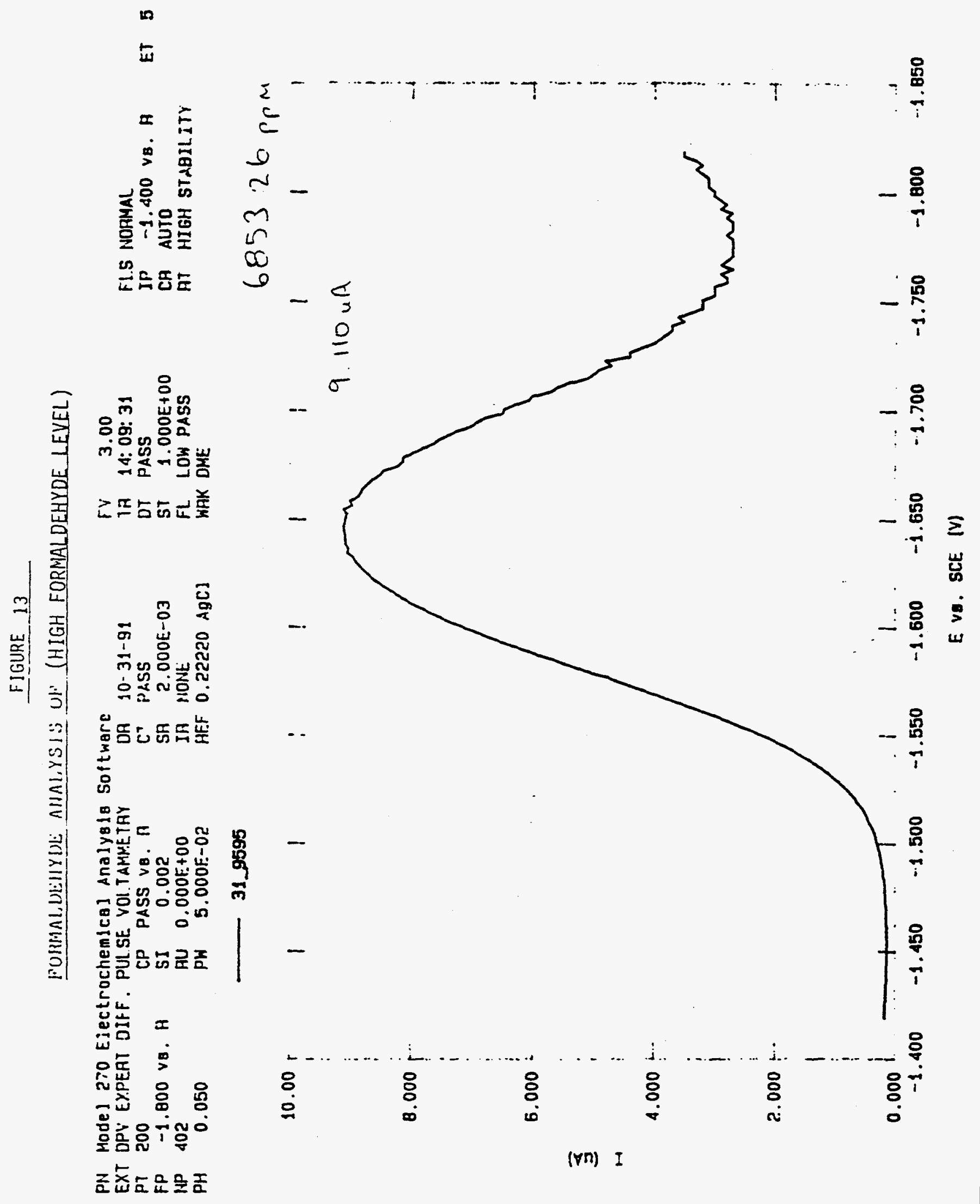


I.UIWIIUL!YY!I, A!ALIY:IS OF (LOW FORMALDEHYDE LEVEL)

PII Mode: 2\%0 Electrochemical Analyoja Softwore

EXT OPY EXPEAT DIFF. PUI SE VO! TAMMETRY

PT 200

FP -1.800 VB, R SI PASS $/ 9$.

MP 402 AU $0.000 E+00$

PH 0.050

OH 10-31.91

CT PLSS

SA $2.000 t-03$

IA HONE

AEF $0.22220 \mathrm{AgCl}$

i: $\quad 3.00$

1A 11: 46: 31

DT PASS

ST $1.000 E+00$

FL L.OH PASS

WAK DME
HLS NOAMÁL.

IP -1.400 v. $A$ ET 5

CA AUTO

RT HIGH STABILITY

\section{9654}
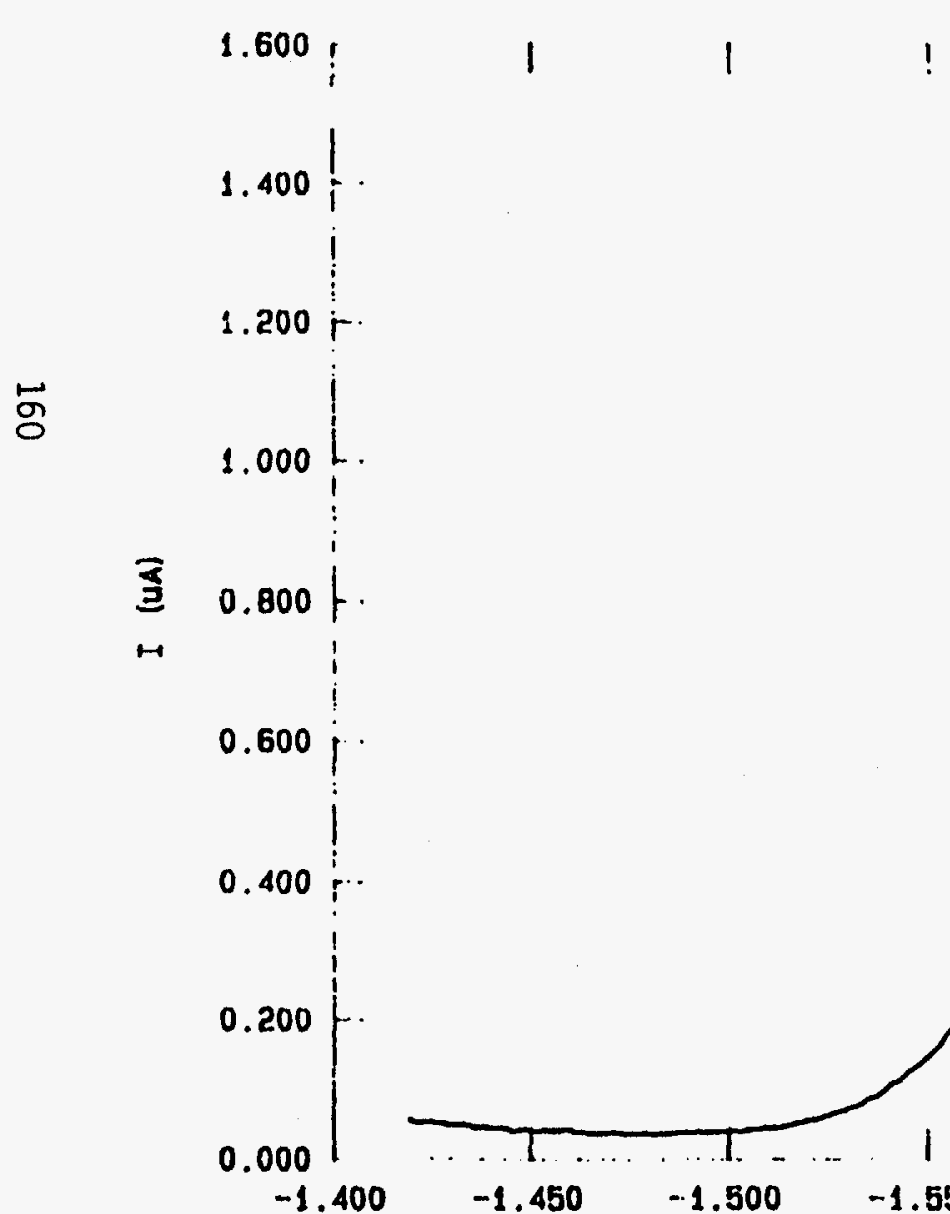

$!$

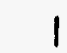

1

1

$1120.9 \mathrm{Pfm}_{1}$ 1 $-1.400$
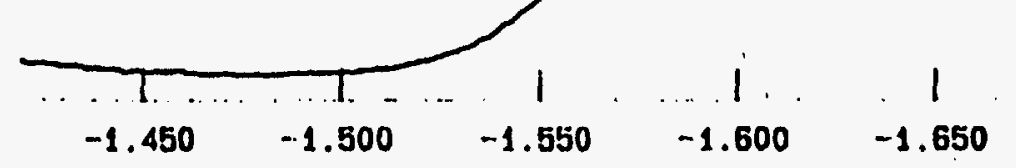

$i$
-1.700

$-1.750$

.1
-1.000

$-1.850$

E V8. SCE (V) 
FIGURE 15

POHAAL,DEIIYDE ANALYYSIS OF (LOW FORMALDEHYDE LEVEL)

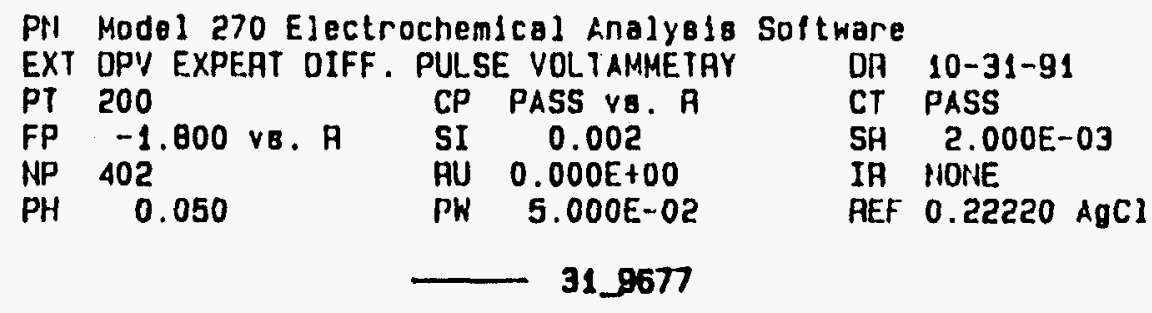

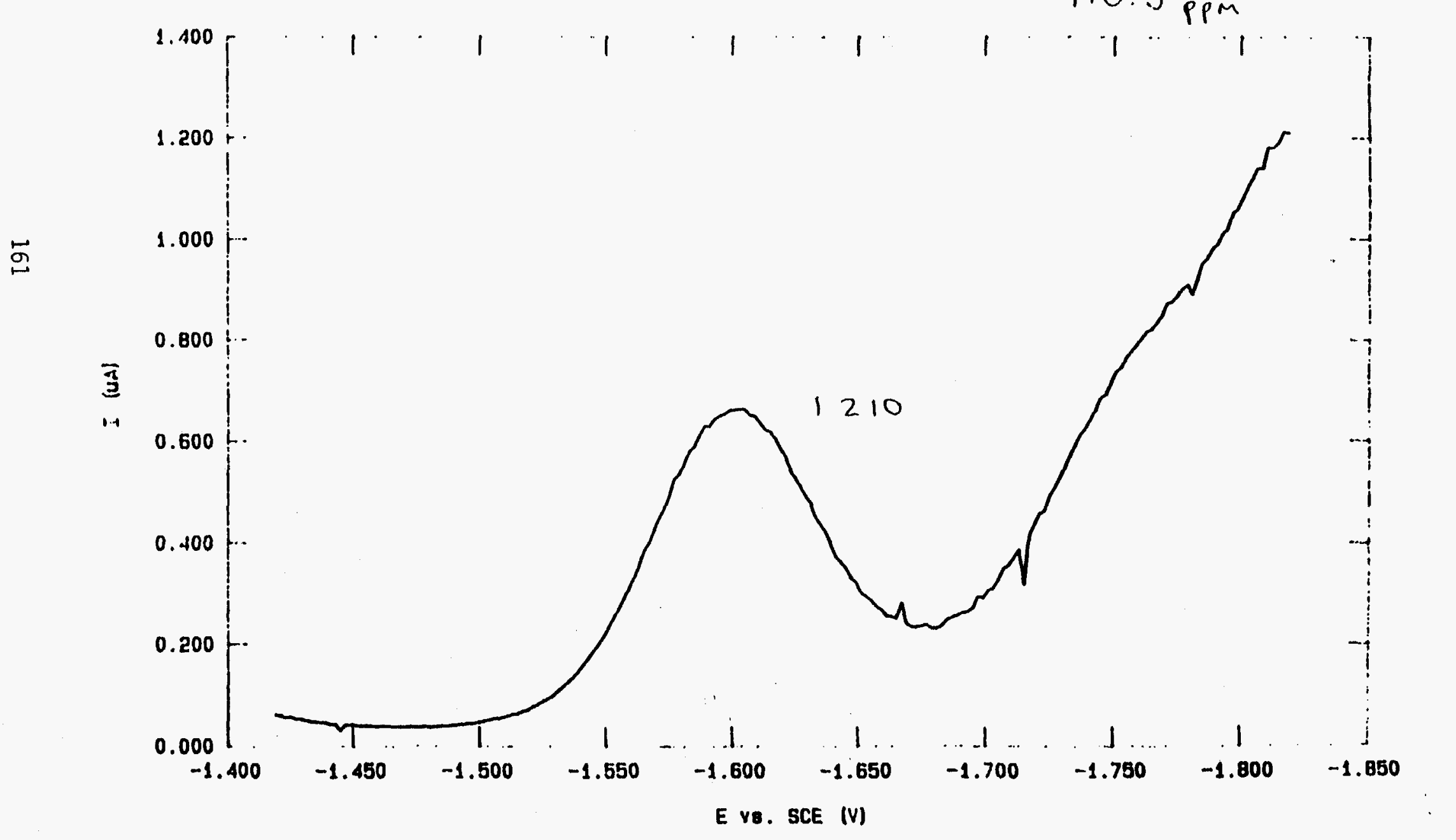


FIGURE 16

POIRMALILEIIYUE NUNI.Y YIS OF (LOW FORMALDEHYDE LEVEL)

PN Model 2:0 Eloctrochemical AnalyBis Softwaro

EXT OPV EXPEAT UIFF. PULSE VOLTAMMETAY OA $10 \cdot 31 \cdot 91$

PT 200

FP $\quad .1 .800$ v8.

NP 402

$\mathrm{PH} \quad 0.050$
CP PASS VB

SI 0.002

RU $0.000 \%+00$

PW 5.000E-02

31.9713

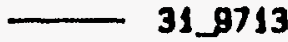

\section{FV $\quad 3.00$ \\ T月 14: 28: 49 \\ OT PASS \\ ST 1.000E +00 \\ FL L.OW PASS \\ WHK DME}

AEF 0.22220 AOC

FLS NOAMAL

IP -1.400 v8. $R$

CA AUTO

HT HIGH STABILITY

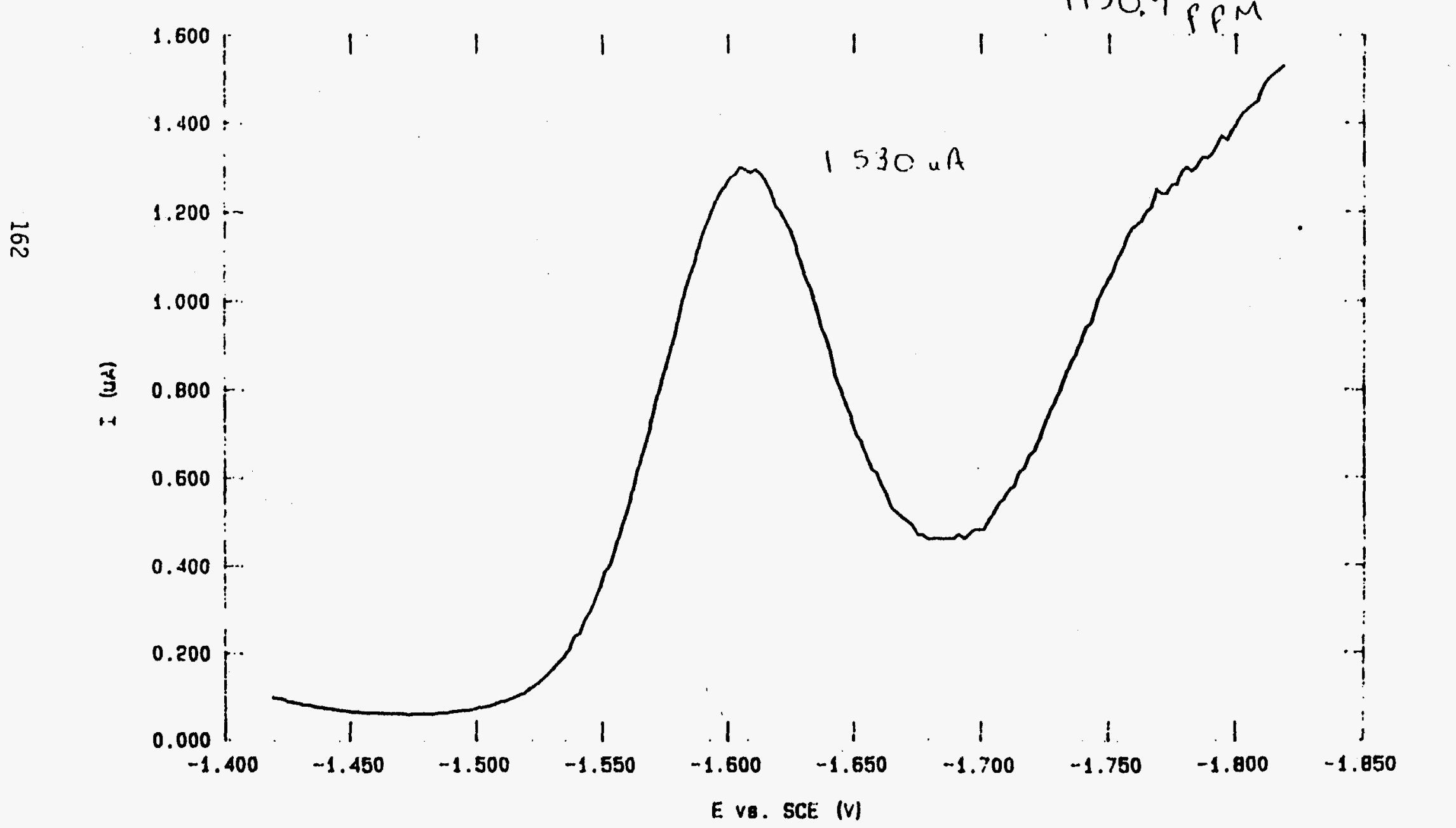




\section{TABLE 5}

\section{METHANE OXIDATIONS}

\begin{tabular}{|c|c|c|c|c|c|c|}
\hline & & $\mathrm{UCT}$ & ole/hr. & $\mathrm{O}_{2} \mathrm{CONV}$ & $\mathrm{CH}_{4} \mathrm{CONV}$. & $\mathrm{CH}_{3} \mathrm{OH}$ \\
\hline & $\mathrm{CO}$ & $\mathrm{CO}_{2}$ & $\mathrm{CH}_{3} \mathrm{OH}$ & $\%$ & $\%$ & $\%$ \\
\hline $\mathrm{Al}_{2} \mathrm{O}_{3}$ & 2.6 & 0.6 & 1.5 & 18 & 1.2 & 32 \\
\hline $\mathrm{SiO}_{2}$ & 3.6 & 0.8 & 3.0 & 37 & 1.9 & 41 \\
\hline $\mathrm{Fe}_{\mathrm{x}}[\mathrm{Fe}] \mathrm{SOD}$ & 6.8 & 1.2 & 7.8 & 65 & 4.0 & 50 \\
\hline MnAPO-X & 6.7 & 1.2 & 8.2 & 65 & 4.2 & 51 \\
\hline
\end{tabular}

A 3/1 mixture of methane and air $\left(5 \% \mathrm{O}_{2}\right)(800 \mathrm{psig})$ was passed over the catalyst (GHSV $\left.=2800 \mathrm{hr}^{-1}\right)$ at $392^{\circ} \mathrm{C}$ and product quantities determined by a combination of gas chromatogaphy and mass spectral analysis. 
TABLE 6

\section{ETHANE OXIDATIONS}

PRODUCTS, mmoles/Hr

\begin{tabular}{|c|c|c|c|c|c|c|c|c|c|c|c|}
\hline Catalyst & Temp & Flow & EtOH & Et\&Ald. & $\mathrm{C}_{2} \equiv$ & $\underline{\mathrm{Me}}$ & $\underline{\mathrm{MeOH}}$ & $\mathrm{CH}_{2} \mathrm{O}$ & $\mathrm{CO}$ & $\mathrm{CO}_{2}$ & $\begin{array}{c}\mathrm{O}_{2} \\
\text { Conv } \\
\end{array}$ \\
\hline $\mathrm{Fe}_{x} \mathrm{FeSOD}$ & 290 & 165 & 0.1 & 0.1 & - & - & 0.4 & $\mathrm{Cr}$ & 0.2 & 0.2 & 10 \\
\hline MnAPO5/47 & 290 & 92 & 1.4 & 0.3 & 0.1 & 1.3 & 4.0 & 1.0 & 3.6 & 0.6 & 94 \\
\hline CoAPSO5/47 & 290 & 102 & 0.05 & 0.05 & 0.03 & $\operatorname{tr}$ & 0.10 & $\operatorname{tr}$ & 0.1 & 0.22 & 15 \\
\hline $\mathrm{Fe}_{x} \mathrm{FeSOD}$ & 300 & 161 & 2.6 & 0.8 & 0 & 2.9 & 8.9 & 3.0 & 8.4 & 1.4 & 99 \\
\hline MnAPO5/47 & 300 & 190 & 3.3 & 0.8 & 0.3 & 2.7 & 11.1 & 3.4 & 2.1 & 1.1 & 97 \\
\hline CoAPSO5/47 & 300 & 176 & 0.15 & 0.12 & 0.04 & $\operatorname{tr}$ & 0.42 & 0.19 & 0.47 & 0.42 & 12 \\
\hline
\end{tabular}

- Continuous oxidation of a 3/1 CH4/air stream at 800 psig over a $1.5 \mathrm{~mL}$ bed of catalyst top loaded into a heated $5 \mathrm{~mL}$ reactor.

b Temperature applied to the reactor skin

c Less than $0.5 \mathrm{mmoles} / \mathrm{hr}$. 
है

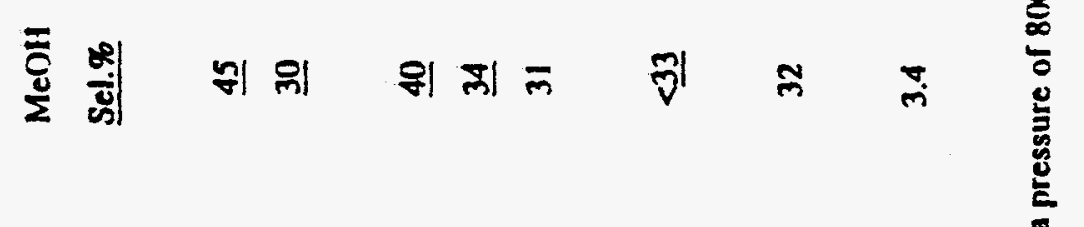

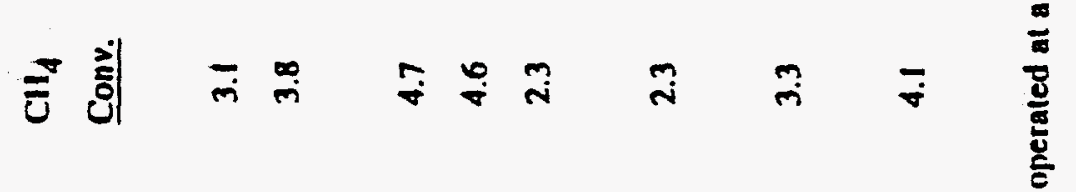

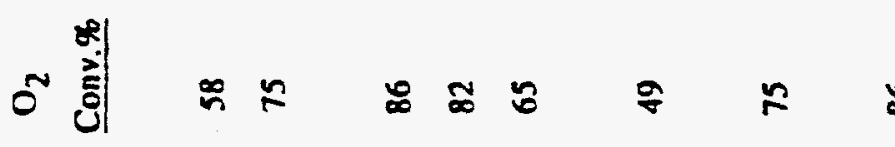

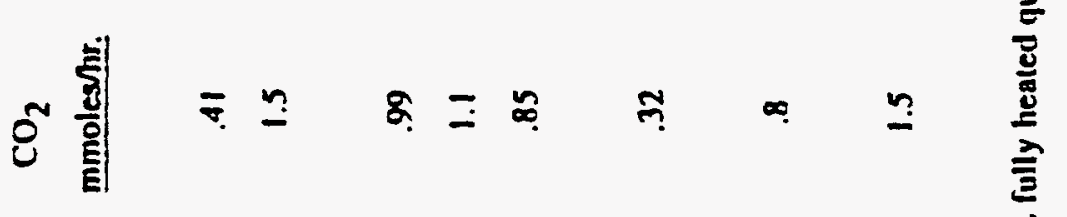

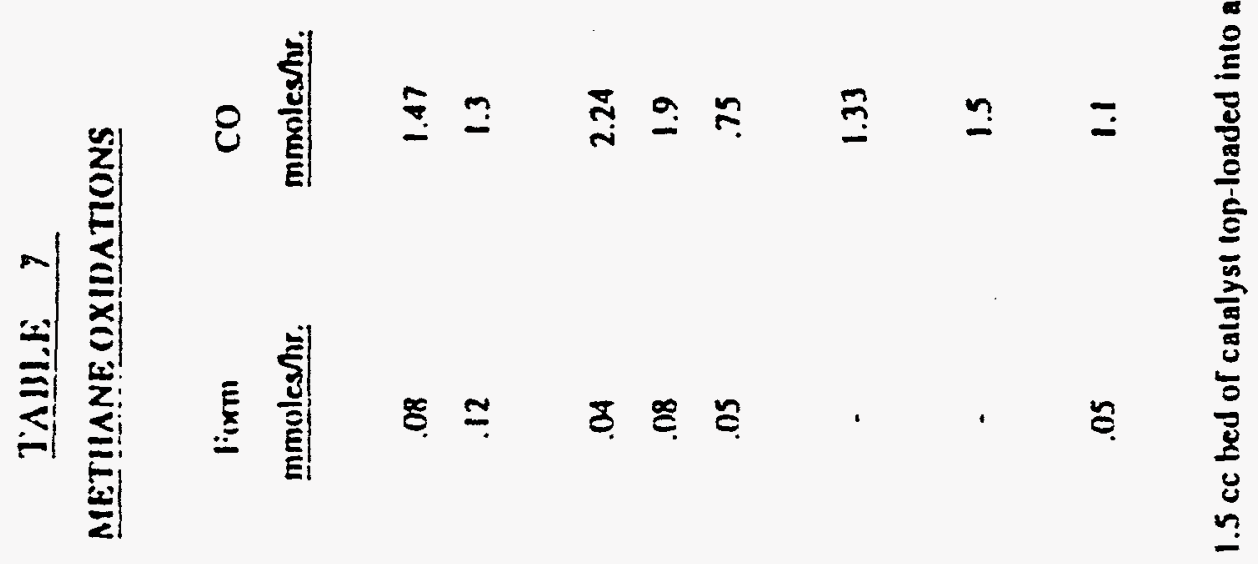

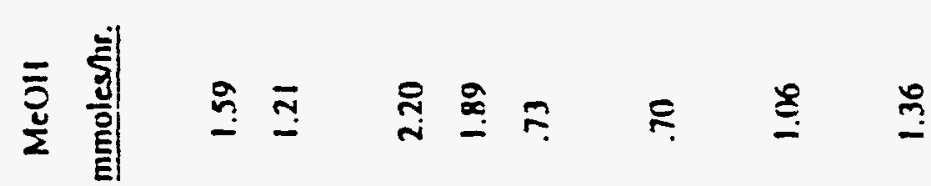

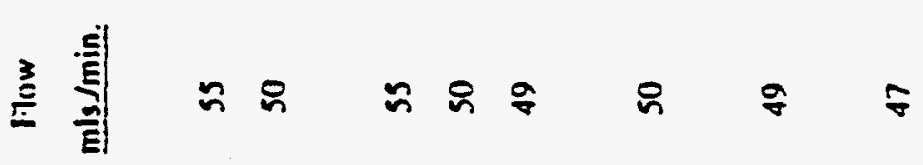

言|

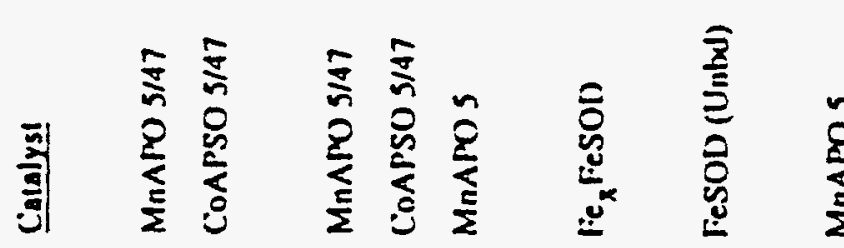




\subsubsection{Methane and Ethane Oxidations Over MnAPO-5}

We have prepared the known manganese substituted aluminophosphate MnAPO-5 and used it as a catalyst for the vapor phase oxidation of methane, Table 8 , and ethane, Table 9. Reactions were conducted in a reactor in which the top third was packed with catalyst and the bottom two thirds was void space. We hoped to generate methyl radicals over the catalyst and allow them to be converted in the void space under conditions in which catalytic decomposition of methanol would be reduced. Methane can be converted to methanol in roughly $50 \%$ selectivity at elevated temperatures over an iron exchanged, iron framework substituted sodalite catalyst, $\mathrm{Fe}_{\mathrm{x}}[\mathrm{Fe}] \mathrm{SOD}$, in this reactor configuration. We have found that methane activation occurs at temperatures about $35^{\circ} \mathrm{C}$ lower with the MnAPO-5 than over $\mathrm{Fe}_{x}[\mathrm{Fe}] \mathrm{SOD}$. Although selectivity is not improved, the finding that the manganese catalyst activates the difficult $\mathrm{C}-\mathrm{H}$ bond of methane more easily than our iron sodalite system is an exciting and potentially important one. We have not yet done the appropriate comparisons between the two catalysts to determine whether the same $\mathrm{C}-\mathrm{H}$ bond activation relationship holds for ethane as well. Data available to date, however, has shown that ethanol yields are higher and $\mathrm{CO}$ make lower over the manganese catalyst.

We have compared results of methane and ethane oxidations over MnAPO-5, MnAPO-5/47 and CoAPSO-5/47 with those using iron sodalite catalysts. While methanol selectivities were not quite as high as in the case of iron sodalite catalysts the reactions could be run at considerably lower temperatures with the $\mathrm{Mn}$ and Co catalysts. The zeolitic matrix into which the iron is substituted can control the oxidation state(s) of the metal which are critical to oxidation catalysis. For this reason, we are interested in making catalysis which can accommodate first-row oxidation-active metals in both the (III) and (II) states as a minimum. We are also interested in zeolitic environments which have very high metal loadings for possible proximate effects in dioxygen binding.

Thus, we felt it imperative to find a zeolitic framework into which we could substitute large amounts of either iron, manganese or cobalt so that a proper comparison could be made. We synthesized MeAPO-11(Me=Fe,Co,Mn) in an attempt to accomplish this. Unfortunately the metal loadings were not as high as those in the sodalite samples which were first investigated but we decided to test them anyway. To date we have completed test runs on FAPO-11 and MnAPO-11. BOTH the iron AND the manganese substituted materials were catalytically active at lower temperatures than was iron sodalite. Both catalysts changed rather quickly while on stream. At $375^{*} \mathrm{C}, 800 \mathrm{psig}$ of $3 / 1$ methane/air, $\sim 1000 \mathrm{gshv}$, the selectivity of FAPO-11 decreased from an initial value of $44 \%$ ( $3-7 \mathrm{hr}$ avg.) to $33 \%$ at $2.4 \%$ methane conversion after less than 20 hours on stream. Under the same conditions using MnAPO-11, selectivity dropped from $46 \%$ (3-7 hr. avg.) to $37 \%$ at $3.7 \%$ methane conversion after about 40 hours on stream. 


\section{METHANE OXIDATIONS OVER MnAPO-5 AND FeX[Fe]SOD}

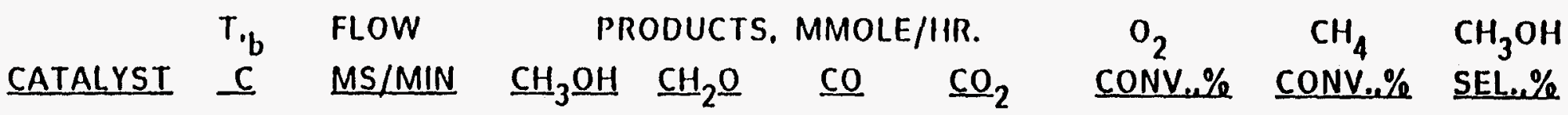

MnAPO-5 375

$\begin{array}{rrrrr}55 & 2.20 & 0.04 & 2.24 & 0.99 \\ 98 & 4.60 & 0.13 & 4.94 & 1.36 \\ 377 & 15.20 & 1.14 & 22.00 & 5.05\end{array}$

$\begin{array}{lll}86 & 4.7 & 40 \\ 93 & 5.2 & 42 \\ 99 & 5.4 & 35\end{array}$

寄 $\mathrm{Fe}_{x}|\mathrm{Fe}| \mathrm{SOD} \quad 4$

$\begin{array}{rrr}50 & 2.22 & \text { NA } \\ 92 & 4.02 & \text { NA }\end{array}$

$2.84 \quad 0.66$

91

5.4

$<39$

$5.32 \quad 0.96$

93

5.3

$<39$

378

19.74

3.14

90

5.5

$<48$

MnAPO-5 365

55

$1.59 \quad 0.08$

1.47

0.41

58

3.1

45

$\mathrm{Fe}_{\mathrm{x}}[\mathrm{Fe}] \mathrm{SOD} \quad 390$

50

$0.70 \mathrm{NA}$

1.33

0.32

49

2.3

$<33$

a A $3 / 1$ methane/air mixture was continuously passed over a $1.5 \mathrm{cc}$ bed of catalyst top-loaded into a 5 $\mathrm{mL}$ fully heated quartz-lined reactor operated at a pressure of $800 \mathrm{psig}$.

b Applied external temperature (to reactor skin) 


\section{ETHANE OXIDATIONS CATALYZED BY Fe FEESOD OR MnAPO-5}

\begin{tabular}{|c|c|c|c|c|c|c|c|c|c|c|c|c|c|}
\hline \multirow[b]{2}{*}{ Catalyst } & \multirow{2}{*}{$\begin{array}{l}T_{b} \\
\underline{C}\end{array}$} & \multirow{2}{*}{$\begin{array}{l}\text { Flow } \\
\mathrm{Ms} / \mathrm{Min}\end{array}$} & \multicolumn{3}{|c|}{ C2's. mmoles/hr. } & \multicolumn{5}{|c|}{ Cl's mmoles $/ \mathrm{hr}$. } & \multirow{2}{*}{$\begin{array}{l}\text { Others } \\
\text { mmoles/ } \\
\text { hr. }\end{array}$} & \multirow{2}{*}{$\begin{array}{l}\mathrm{O}_{2} \\
\text { Used } \\
\%\end{array}$} & \multirow{2}{*}{$\begin{array}{r}\mathrm{C}_{2} \mathrm{H}_{6} \\
\text { Conv. } \\
\% \\
\end{array}$} \\
\hline & & & $\mathrm{CH}_{3} \mathrm{CH}$ & $\mathrm{H} 3 \mathrm{CHO}$ & $\mathrm{CH}_{2} \equiv \mathrm{CH}_{2}$ & $\mathrm{CH}_{4}$ & $\mathrm{CH}_{3} \mathrm{OH}$ & $\mathrm{CH}_{2} \mathrm{O}$ & $\mathrm{CO}$ & $\mathrm{CO}_{2}$ & & & \\
\hline $\left.\mathrm{Fe}_{x} \mid \mathrm{Fe}\right] \mathrm{SOD}$ & 290 & 165 & 0.1 & 0.1 & c & c & 0.4 & tr & 0.2 & 0.2 & c & 10 & 0.2 \\
\hline MnAPO -5 & 290 & 92 & 1.4 & 0.3 & 0.1 & 1.3 & 4.0 & 1.0 & 3.6 & 0.6 & 0.3 & 94 & 2.9 \\
\hline $\mathrm{Fe}_{x}|\mathrm{Fe}| \mathrm{SOD}$ & 300 & 161 & 2.6 & 0.8 & 0 & 2.9 & 8.9 & 3.0 & 8.4 & 1.4 & 0.2 & 99 & 3.7 \\
\hline MnAPO-5 & 300 & 190 & 3.3 & 0.8 & 0.3 & 2.7 & 11.1 & 3.4 & 2.1 & 1.1 & 0.4 & 97 & 3.5 \\
\hline $\left.\mathrm{Fe}_{\mathbf{x}} \mid \mathrm{Fe}\right] \mathrm{SOD}$ & 315 & 386 & 3.0 & 1.9 & 4.1 & 10.0 & 18.1 & 14.0 & 25.1 & 3.3 & 0.5 & 93 & 3.7 \\
\hline MnAPO-5 & 300 & 406 & 6.2 & 1.7 & 1.0 & 9.1 & 24.9 & 8.3 & 10.5 & 3.3 & 0.8 & 98 & 4.2 \\
\hline
\end{tabular}

a) Continuous Oxidation of a $3 / 1 \mathrm{CH}_{4} /$ air stream at 800 psig over a $1.5 \mathrm{~mL}$ bed of catalyst top loaded into a heated $5 \mathrm{~mL}$ reactor.

b) Temperature applied to the reactor skin. 


\subsubsection{Methane Oxidation Over [Fe]ZSM-5}

Table 10 presents some of the results of the testing of the sodium exchanged pentasil zeolite, [Fe]ZSM-5, the synthesis and characterization of which was described in an earlier progress report. It should be noticed that the framework iron content of this catalyst was far lower than that of Fe[SOD] and the methanol production was less than half of that produced using [Fe]SOD.

\subsubsection{Reactions catalyzed by $\mathrm{O} .5 \% \mathrm{CrO}_{3} / \mathrm{SiO}_{2}$}

We have found that low levels of chromia on silica prepared according to the method of McDaniels, et al., (21) which contains surface chromium(VI) oxo groups having the structure, I, below, is active for the oxidation of methane to methanol.

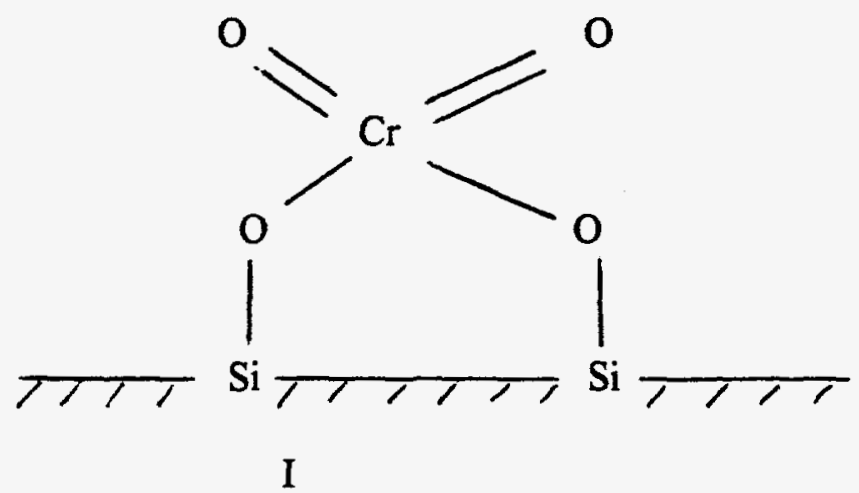

Initial results of the testing of this catalyst were obtained in a downflow reactor under conditions which were not optimized for high methanol selectivity.

Table 11 presents some preliminary results of tests over this "surface-oxo" catalyst which were compared to tests of both [Fe]SOD and $3 \% \mathrm{CrO}_{3} / \mathrm{SiO}_{2}$ under the same conditions. The chromium oxo catalyst compared favorably to iron sodalite in methanol selectivity and was quite superior to heavy loadings of $\mathrm{CrO}_{3}$ which have been shown to be less active coatings of $\mathrm{CrO}_{3}$ and di- and polychromates. It is of interest that defined chromium oxo species anchored to the oxidic surface structure have methane-to-methanol activity and catalysts of this type will be explored in more detail in the future.

\subsubsection{Catalyst Testing in a CSTR}

We have performed work on the synthesis of catalyst candidates based on binuclear metal-ion sites isolated in silicometallate matrices, and we have begun preliminary catalyst testing of these candidate solids in our gold plated CSTR reactor with Robinson-Mahoney internals for their ability to catalyze methanol synthesis under oxygen limited conditions. 


\section{TABLE 10}

\section{OXIDATION OF METHANE ${ }^{a}$ OVER FE[ZSM-5 $]^{b}$ IN THE VAPOR PHASE}

\begin{tabular}{|c|c|c|c|c|c|c|c|}
\hline BED. $T^{\cdot C}$ & GHSV. & PRODU & TS, $n$ & les/Hr. & $\mathrm{CH}_{3} \mathrm{OH}$ & $\mathrm{CH}_{4} \%$ & $\mathrm{O}_{2}$ \\
\hline${ }^{\circ} \mathrm{C}$ & $\underline{H R^{-1}}$ & $\mathrm{CH}_{3} \mathrm{OH}$ & $\mathrm{CO}$ & $\mathrm{CO}_{2}$ & SEL.\% $\%$ & CONV. $\%$ & CONV. $\%$ \\
\hline 410 & 12,000 & 0.1 & 0.9 & 9.3 & 1.0 & 0.6 & 15 \\
\hline 418 & 12.000 & 0.2 & 0.9 & 9.1 & 2.0 & 0.6 & 15 \\
\hline 428 & 12,000 & 17.4 & 60.8 & 17.6 & 17.4 & 6.2 & 97 \\
\hline 430 & 12.000 & 15.4 & 67.2 & 13.4 & 15.0 & 6.3 & 97 \\
\hline
\end{tabular}

a $P=800$ psig, 3.1 methane: are Quartz lined reactor described in earlier reports.

b $7.58 \%$ iron. $0.07 \%$ Al

c Measured at mid-bed. 


\section{OXIDATION OF METHANE OVER CHROMIA ON SILICA"}

\begin{tabular}{|c|c|c|c|c|c|c|c|}
\hline & & $\begin{array}{c}\text { Applied } \\
T \\
{ }^{\circ} \mathrm{C} \\
\end{array}$ & $\begin{array}{l}\text { Gas } \\
\text { Flow. } \\
\mathrm{m} / \mathrm{s} / \mathrm{Hr} .\end{array}$ & $\begin{array}{c}\mathrm{O}_{2} \\
\text { Converted, } \\
\% \\
\end{array}$ & $\begin{array}{c}\mathrm{CH}_{4} \\
\text { Converted. } \\
\% \\
\end{array}$ & $\begin{array}{l}\mathrm{CH}_{3} \mathrm{OH} \\
\text { Produced. } \\
\text { mmoles } / \mathrm{Hr} \text {. }\end{array}$ & $\begin{array}{c}\text { Methanol } \\
\text { Selectivity, } \\
\%\end{array}$ \\
\hline Iron & Sodalite & 400 & 800 & 15 & 0.9 & 3.6 & 24 \\
\hline $3 \%$ & $\mathrm{CrO}_{3} / \mathrm{SiO}_{2}$ & 400 & 800 & 16 & 0.6 & 5.6 & b \\
\hline & " & 420 & 800 & 20 & 0.7 & 5.1 & b \\
\hline & " & 430 & 800 & 22 & 0.7 & 4.6 & b \\
\hline & - & 400 & 400 & 18 & 0.7 & 2.5 & b \\
\hline & $"$ & 400 & 200 & 22 & 0.7 & 0.9 & b \\
\hline & $n$ & 375 & 200 & 22 & 0.9 & 1.3 & $b$ \\
\hline & - & 350 & 100 & 22 & 0.9 & 0.3 & b \\
\hline & " & 300 & 50 & 18 & 0.4 & 0.02 & $b$ \\
\hline Iron & Sodalite & 300 & 50 & 0 & 0 & 0 & - \\
\hline $0.5 \%$ & $\% \mathrm{CrO}_{3} / \mathrm{SiO}_{2}$ & 390 & 800 & 15 & 0.6 & 3.9 & 41 \\
\hline & - & 400 & 800 & 29 & 1.8 & 10.9 & 38 \\
\hline & - & 410 & 800 & 38 & 2.0 & 11.8 & 38 \\
\hline & - & $420^{c}$ & 800 & 90 & 5.4 & 18.5 & 22 \\
\hline & - & 350 & 100 & 15 & 0.4 & 0.3 & 34 \\
\hline & - & 300 & b & b & b & $b$ & b \\
\hline
\end{tabular}

- Continuous oxidation of methane (800 psig. 31 . methane air) over one cc of catalyst. Results reported are average of four hourly samples taken after a two-hour line-out period.

b Data will become available on completion of gas analysis.

c Exotherm caused internal temperature of $442^{*}$ during reaction. 
Previous work in packed bed reactors along with modelling studies lead us to conclude that we could not appropriately measure the exact reaction temperature (as opposed to the catalyst bed temperature) and that we could not disentangle the degree of backmixing from the residence time in the reactor. Use of the CSTR reactor (Figure 17) has enabled us to precisely determine the reaction temperature and to separate residence time (GHSV) effects from those which are related to the degree of backmixing. In parallel to the experimental effort, we have modified a version of our computer simulation of the homogeneous kinetics to take into account backmixing effects. This model has been employed to identify those factors which might be altered in a CSTR reactor relative to a packed bed.

We chose to experimentally examine a "low" temperature region with hopes of better discriminating a catalyzed process in parallel to the homogeneous vapor phase non-catalyzed processes leading to methanol, since the non-catalyzed processes are relatively slow at low temperature. We fixed $\mathrm{T}=400^{\circ} \mathrm{C}, \mathrm{P}=900 \mathrm{psig}$, feed $=3: 1 \mathrm{CH}_{4}: \mathrm{AIR}$. Using silica gel packing which should account for peroxide quenching effects but not show further catalysis, we studied the effect of varying flow rate, reactor bypass side arm utilization, and side arm-reactor outlet mixing temperature. A statistically significant number of runs were conducted for each process variable measurement; only data of acceptable carbon balances utilized; and error limits representing a $95 \%$ confidence interval were computed for each data set.

We found that a high flow rate and high stirring rate, i.e. a fully backmixed reaction, were the most favorable operating conditions. This finding corresponds to our qualitative conclusion frommodelling studies that oxygen consumption (the limiting reagent) should decrease as backmixing increases when approaching $100 \%$ oxygen conversion.

We had previously noted that utilization of a particular reactor design feature appended to a packed bed reactor led to enhanced methanol selectivity under low flow conditions. This design feature consisted of a side-arm on the packed bed reactor connecting an unoccupied fore-chamber ahead of the catalyst bed to the effluent stream exiting from the catalyst. We believe that the conditions under which this "bypass reactor" operated resulted in backmixing of intermediates desorbed from the catalyst surface into the unoccupied void space ahead of the catalyst where a further reaction could ensue without either quenching or decomposition by the solid packing. The bypass served to draw off reaction products from the region not occupied by catalyst. Unfortunately, we could not independently control the degree of backmixing without altering the flow rate.

We have confirmed the beneficial effect of the bypass on the CSTR reactor in fully backmixed operation using only silica gel packing. Here flow rate and the degree of backmixing (stirring rate) are independent process variables. Methanol selectivity initially increases as the micrometering valve of the side arm is opened. With a high degree of confidence, we can conclude that opening the side arm results in significant enhancement of the observed methanol selectivity and that there is an optimum opening. A U.S. patent claiming this reactor design benefit for catalyzed reactions has been filed. 
FIGURE

$\mathrm{CH} 4$

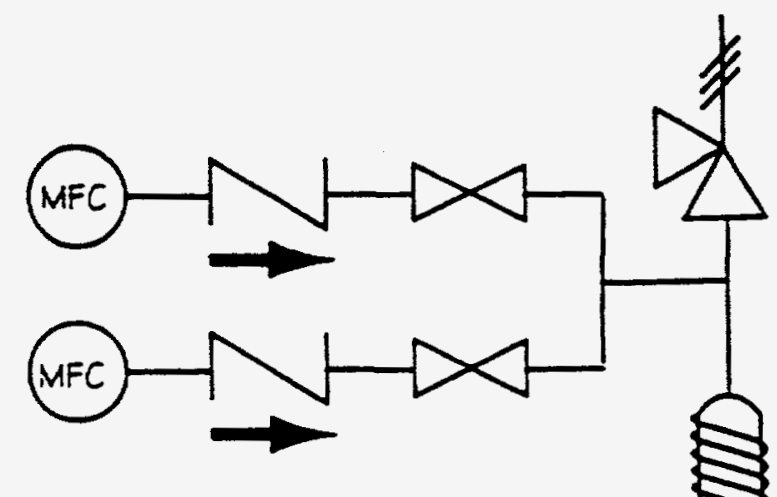

PREHEATER

$\mathrm{O}_{2}$ or
AIR

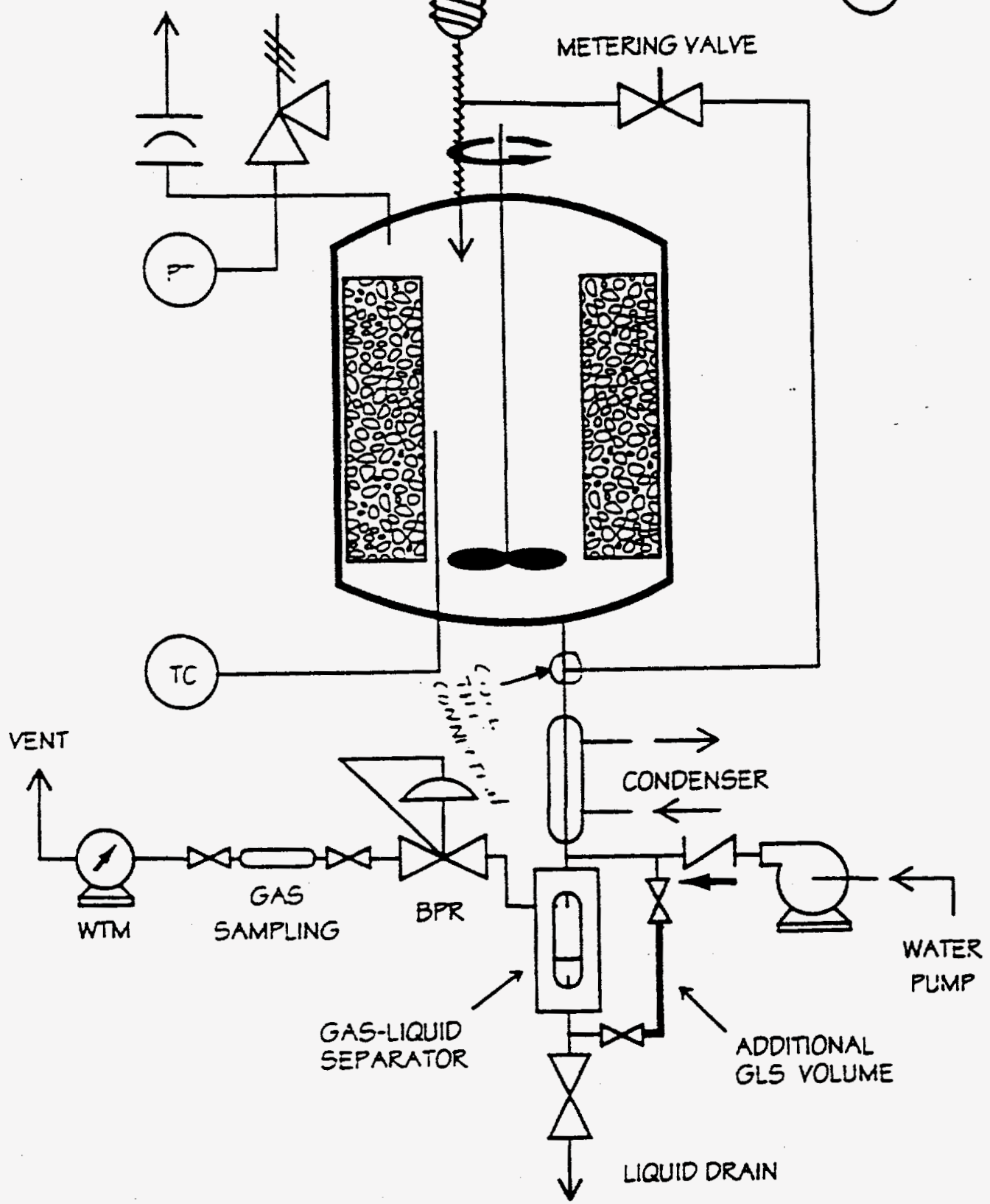

METERING YALVE 
We have now also discovered (Table 12) that deliberate cooling of the connection between the side-arm bypass and the main reactor effluent line results in still further improvement in methanol selectivity when the side arm is at the optimal opening. Although the detailed mechanistic chemistry which is responsible for these observations is not clear, it is interesting to speculate that the activation energy for decomposition of intermediates potentially leading to methanol (or for decomposition of methanol itself) to $\mathrm{CO}_{\mathrm{x}}$ must have a higher activation energy than do the pathways leading to methanol synthesis from the common intermediate. We do not yet know if the cooling effect improves selectivity in the absence of the side arm. If it does, a "quick quench" reactor may be desired to optimize yield of methanol.

We have begun to test a series of catalyst candidates based on ion exchanged silicoferrates. The first of the series is [Fe]SOD with no additional exchange but of a different synthetic batch than found to be active in packed bed reactors previously. Although the present results do not represent optimum conditions for yield maximization, the data has been measured under rigorously controlled conditions so that observed differences from control runs can be ascribed to reactor packing. The bypass line was utilized at an opening that had previously been found to be optimal for silica gel; cooling of the bypass intersection was effected to the same degree in both the control runs and in the actual catalyst runs.

Initial use of a calcined $[\mathrm{Fe}] \mathrm{SOD}$ at $400^{\circ} \mathrm{C}$ resulted in methanol yields indistinguishable from those measured over silica gel. An attempt to activate the material by simply heating to $450^{\circ} \mathrm{C}$ followed by a 400 degree extended run ( 10 hours) failed to boost methanol selectivity or conversion. However, subsequent injection of several $\mathrm{ml}$ of water into the system at $450^{\circ} \mathrm{C}$ to steam-treat the [Fe]SOD followed by operation at 400 degree did result in activation of the catalyst. Over a period of about 4-5 hours, the methanol selectivity improved then lined out and remained steady over the next 8 hours on-stream. About 40 gas or liquid aliquots were gathered over this time period and subsequently analyzed. Mean data and error limits representing 95\% confidence are listed in Table 12 for the activated and lined out [Fe]SOD compared to silica gel. Comparable experiments over silica gel indicated line out time to be certainly within 1 hour and possibly within an even shorter time. These observations are consistent with the hypothesis that some framework iron must move to exchange sites for the catalyst to become active. Once established, the iron-iron pair sites catalyze methanol formation, even at the relatively low temperature of $400^{\circ} \mathrm{C}$. 
TABLE 12

\section{METHANE OXIDATION IN A CSTR}

Silica Gel Gold/CSTR Methane:Air 3:1

\begin{tabular}{|c|c|c|c|c|c|c|c|c|c|c|}
\hline \multirow[b]{2}{*}{ Feed } & \multirow[b]{2}{*}{$\underline{\text { Stir Rate }}$} & \multirow[b]{2}{*}{ Temp } & \multirow[b]{2}{*}{ Pres } & \multirow[b]{2}{*}{ Sidearm } & \multirow[b]{2}{*}{ T. Sidearm } & \multicolumn{2}{|c|}{ Conversion, $\%$} & \multicolumn{3}{|c|}{ Selectivity, \% } \\
\hline & & & & & & C & $\mathrm{O}_{2}$ & $\mathrm{CH}_{3} \mathrm{OH}$ & $\mathrm{CO}$ & $\mathrm{CO}_{2}$ \\
\hline$g h$ & 2000 RPM & 400 & 900 & Closed & $100-120$ & $5.11 \pm .17$ & $95.36 \pm 1.71$ & $9.77 \pm 1.89$ & $50.77 \pm 2.33$ & $39.46^{-t}$ \\
\hline igh & 2000 RPM & 400 & 900 & .5 Rev. & $100-120$ & $5.11 \pm .11$ & $96.74 \pm 1.13$ & $14.44 \pm .76$ & $50.15 \pm 1.91$ & $35.61 \pm 2.06$ \\
\hline gh & 2000 RPM & 400 & 900 & .5 Rev. & $56-64$ & $5.78 \pm .18$ & $95.26 \pm 1.77$ & $22.71 \pm 1.18$ & $43.99 \pm 1.88$ & $33.29 \pm 2.28$ \\
\hline w & 2000 RPM & 400 & 900 & .5 Rev. & $6-9$ & $6.26^{ \pm} .09$ & $96.46 \pm 3.71$ & $18.74 \pm .25$ & $43.58^{ \pm} 1.04$ & $37.68^{ \pm} .88$ \\
\hline w & NONE & 400 & 900 & .5 Rev. & $7-15$ & $6.45^{ \pm} .07$ & $96.36^{ \pm} 1.14$ & $19.47^{ \pm} 1.59$ & $47.03^{ \pm} 3.26$ & $33.62^{ \pm} 2.52$ \\
\hline igh & 2000 RPM & 400 & 900 & 1 Rev. & $100-120$ & $5.17 \pm .15$ & $96.08 \pm 2.38$ & $13.23 \pm 1.85$ & $48.39 \pm 2.01$ & $38.38 \pm 1.51$ \\
\hline a & 2000 RPM & 400 & 900 & 2 Rev. & $100-120$ & $5.18^{ \pm} .09$ & $94.98^{ \pm} 1.47$ & $12.34^{ \pm} .43$ & $50.85^{ \pm} 1.08$ & $36.81 \pm 1.33$ \\
\hline
\end{tabular}




\section{TABLE13}

\section{COMTARISON OF AN ACTIVATED IFEISOD AND SILICA GELIN A CSTR REACTOR AT 400*C UNDER FULLY BACKMIXED CONDITIONS WITH THE BYPASS OPEN}

\begin{tabular}{ccccccc} 
& & \multicolumn{3}{c}{ PRODUCT SELECTIVITY \% } & \multicolumn{2}{c}{ CONVERSION, \% } \\
PACKING & SIDEARM & $\underline{\mathrm{CH}_{3} \mathrm{OH}}$ & $\underline{\mathrm{CO}}$ & $\mathrm{CO}_{2}$ & $\underline{\text { CARBON }}$ & $\mathrm{Q}_{2}$ \\
Silica Gel & $82^{\circ} \mathrm{C}$ & 21.19 & 43.7 & 35.1 & 5.34 & 96.6 \\
[Fe]SOD & $80.9^{\circ} \mathrm{C}$ & $31.81 \pm 1.21$ & $40.2 \pm .1 .1$ & $28.0 \pm .6$ & $6.32 \pm 1.1$ & $92.4 \pm 1.5$
\end{tabular}

\subsubsection{Methane Oxidations Under Different Conditions}

In past reports we have stressed the critical nature of the methane oxidation conditions on methanol selectivity. We have shown how under the same conditions of temperature, pressure and flow rate, selectivities of the iron sodalite catalyzed oxidation of methane could vary from under $20 \%$ in a back mixed spinning basket reactor to under $30 \%$ in a fully packed down-flow reactor to around $50 \%$ in a top-loaded down-flow reactor to $70 \%$ in a flow reactor aided by product removal by reactive distillation. Table 14 shows how changes in feed mixture, catalyst loading and flow rate in yet another reactor design described below can also affect product selectivity. A combination of higher flow rate, higher oxygen partial pressure, different configuration and the presence of a glass frit all may contribute to poorer methanol selectivity. Table 15 shows how selectivity varies dramatically with pressure under one set of conditions and Figure 18 shows the same phenomenon under somewhat different conditions.

\subsubsection{Experimental Details of Methane Oxidations}

Some recent experimentation in our laboratory was conducted under reactor test condiditons including relatively high proportions of air in the feed. These runs were conducted in a quartz-lined downflow packed bed reactor in which $2-4 \mathrm{ml}$ of an 18/35 mesh solid catalyst were packed over a quartz frit. About $4 \mathrm{ml}$ of empty space preceded the catalyst in the heated zone and abut $5 \mathrm{ml}$ of empty space was present below the frit in the heated zone. A thermocouple was inserted into the middle of the packed bed in most runs, but this was removed in others to ensure no contribution by the thermocouple material to catalysis. Prior to the reactor, methane and air were mixed and preheated in a hastelloy C CSTR operated at an impeller speed of several thousand RPM and $300^{\circ} \mathrm{C}$. All runs were conducted at $820+/-5$ psig. 
TABLE 14

OXIDATIONS OF METHANE $\Lambda$ T HIGH $O_{2}$ AND GISV

\begin{tabular}{|c|c|c|c|c|c|c|c|c|}
\hline CATALYST & $\begin{array}{l}\text { MLS } \\
\text { CAT }\end{array}$ & $\begin{array}{l}\text { CII4/ } \\
\text { AIR }\end{array}$ & $\begin{array}{l}\text { GHSV } \\
\text { (TOT. } \\
\text { YOL.) }\end{array}$ & $\begin{array}{l}\text { GHSV } \\
\text { (CAT) }\end{array}$ & $\mathbf{T}^{0} \mathrm{C}$ & $\begin{array}{c}\mathrm{CH}_{4} \\
\text { CONv.,\% }\end{array}$ & $\begin{array}{l}\mathrm{O}_{2} \\
\text { CONV.,\% }\end{array}$ & $\begin{array}{l}\mathrm{CH}_{3} \mathrm{OH} \\
\mathrm{SEL} .2 \%\end{array}$ \\
\hline \multirow[t]{4}{*}{ [Fe]SOD } & 1.3 & 2.23 & 2.724 & 14.668 & 414 & 6.7 & 93 & 16 \\
\hline & 1.3 & 2.23 & 2.724 & 14,668 & 414 & 5.6 & 94 & 15 \\
\hline & 2.7 & 2.23 & 2,724 & 7,063 & 405 & 4.8 & 79 & 22 \\
\hline & 2.7 & 2.23 & 2.724 & 7,063 & $\mathbf{3 7 5}$ & 0.6 & 64 & $\mathbf{9}$ \\
\hline $\left.\mathrm{Fe}^{2+} \mid \mathrm{Fe}\right] \mathrm{SOD}$ & $\mathbf{3 . 3}$ & 2.23 & 2,724 & 6.779 & 408 & 6.8 & 99 & 20 \\
\hline MnAPO-5 & $\mathbf{5 . 0}$ & 2.23 & 2.724 & 3,814 & 380 & 4.9 & 96 & 3 \\
\hline \multirow{2}{*}{ Fe-FPc//Fe]SOD } & 2.1 & 2.23 & 2,724 & 9,080 & 407 & $\mathbf{5 . 8}$ & 94 & 15 \\
\hline & 2.1 & 2.23 & 2,724 & 9,080 & 407 & 5.4 & 96 & 15 \\
\hline \multirow{2}{*}{$\mathrm{Fe}-\mathrm{FPc} / \mathrm{SIO}_{2}$} & 3.1 & 2.23 & 2.724 & 6,151 & 408 & 3.4 & 72 & 22 \\
\hline & 3.1 & 2.23 & 2,724 & 6,151 & $\mathbf{3 7 5}$ & 1.3 & 62 & 12 \\
\hline \multirow[t]{2}{*}{$\mathrm{SlO}_{2} \mathrm{gel}$} & $\mathbf{3 . 2}$ & 2.23 & 2,724 & 5,959 & 405 & 3.0 & 78 & 30 \\
\hline & 3.2 & 2.23 & 2,724 & $\mathbf{5 . 9 5 9}$ & $\mathbf{3 8 0}$ & 2.1 & 22 & 0.4 \\
\hline
\end{tabular}

asee experimental detail. 


\section{TABLE 15}

\section{EFEECT OF PRESSURE ON METAFNE OXIDATIONS}

CATALYZED BY FeISOD] IN A KUNG REACTOR

$\begin{array}{lcc}\mathrm{T}^{\circ} \mathrm{C} & 414 & 414 \\ \text { P,psig } & 803 & 205 \\ \mathrm{O}_{2} \mathrm{CONV} . & 96.0 \% & 9.2 \% \\ \mathrm{CH}_{4} \text { CONV. } & 5.32 & 0.39 \\ \mathrm{CH}_{3} \mathrm{OH} \text { SEL. } & 23.8 & 9.0 \\ \mathrm{CO} \mathrm{SEL} . & 60 & 5.7 \\ \mathrm{CO}_{2} \text { SEL. } & 16.2 & 85.3 \\ \mathrm{CBALANCE} & 97.6 \% & 100.6 \%\end{array}$

${ }^{2}$ Catalyst, $1.39 \mathrm{ml}$, top-loaded into a quartz-lined reactor $(\mathrm{D}=0.7 \mathrm{~cm})$. Methane/air (3:1)fed at 190m NTP (GHSV (Total Vol.) $\left.=1815 \mathrm{~h}^{-1}\right)$. 


\section{P DEPENDENCE OF CH3OH YIELD}

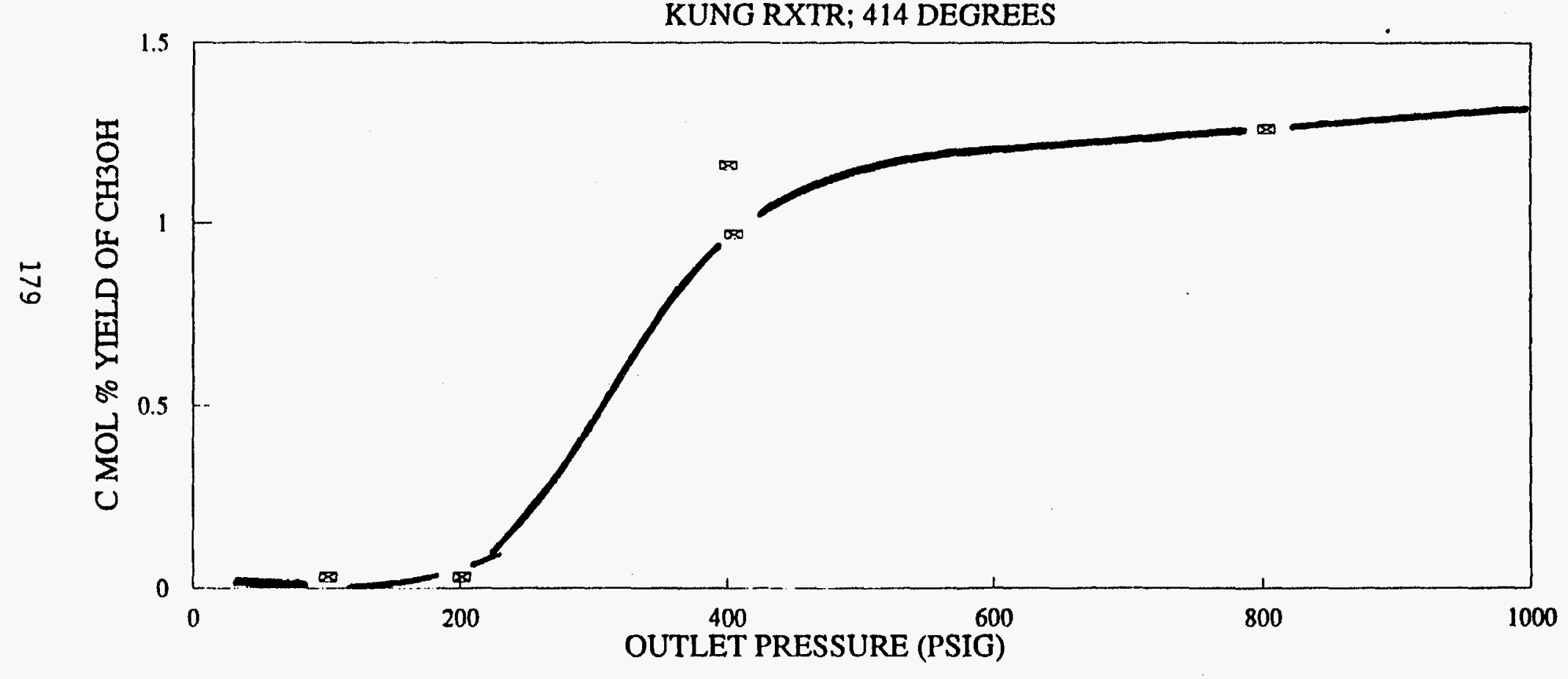


Control runs were conducted with silica gel packing in the reactor which was expected to provide surface for alkylperoxy radical quenching but not to contribute to catalysis beyond the homogeneous thermal reaction. Several batches of [Fe]SOD with and without further $\mathrm{Fe} 2+$ exchange were examined as well as MeAPO-type molecular sieves containing manganese, and a perfluorophthalocyanineiron(II) compound supported on either silica or [Fe]SOD. Data measured after 300-400 minutes on-stream are presented in Table 14. Each data point represents the mean of at least four gas analyses and three liquid analyses; additional data from these runs including 21 additional analyses for each run are archived at the Sun Laboratories. Since in all but one case, the catalysts had not been aged on-stream, these data represent minimum performance for the [Fe]SOD cases and perhaps also for the MnAPO catalysts.

Comparison of the present experimental results to lower oxygen content runs leads one to the general conclusion that methane conversion is not necessarily increased by a higher partial pressure of oxygen in the feed. Despite higher oxygen partial pressure, conversions of about $2-6 \%$ were observed in the present runs, on average, just as had been observed for 3:1 methane:air mixtures previously. Methanol selectivity was found to be generally lower than in earlier runs at higher methane concentration, however. Considering the strong tie between conversion and selectivity, i.e., at constant oxygen conversion, the more methanol produced relative to $\mathrm{CO}_{2}$, the greater the conversion of methane, it is not surprising that methane conversion was not observed to be higher in our recent runs since modelling studies predict that $\mathrm{CO}_{2}$ formation is increased at the expense of methanol by higher oxygen concentration.

Another area of continuing interest is formaldehyde production. Using a differential pulse polarographic analytical technique, we have examined the formaldehyde yield from a number of methane oxidation runs reported above and have found a correlation between yields of methanol and formaldehyde in most runs examined (Figure 19). This suggests that a common progenitor to methanol and formaldehyde might exist, in agreement with our homogeneous reaction kinetic model prediction. The formaldehyde yield was found to be roughly 10-15 times smaller than the methanol yield in our runs, also consistent with semiquantitative model predictions made earlier. 
FIGURE 19

cllsoll yleld vo. Cll-u yleld

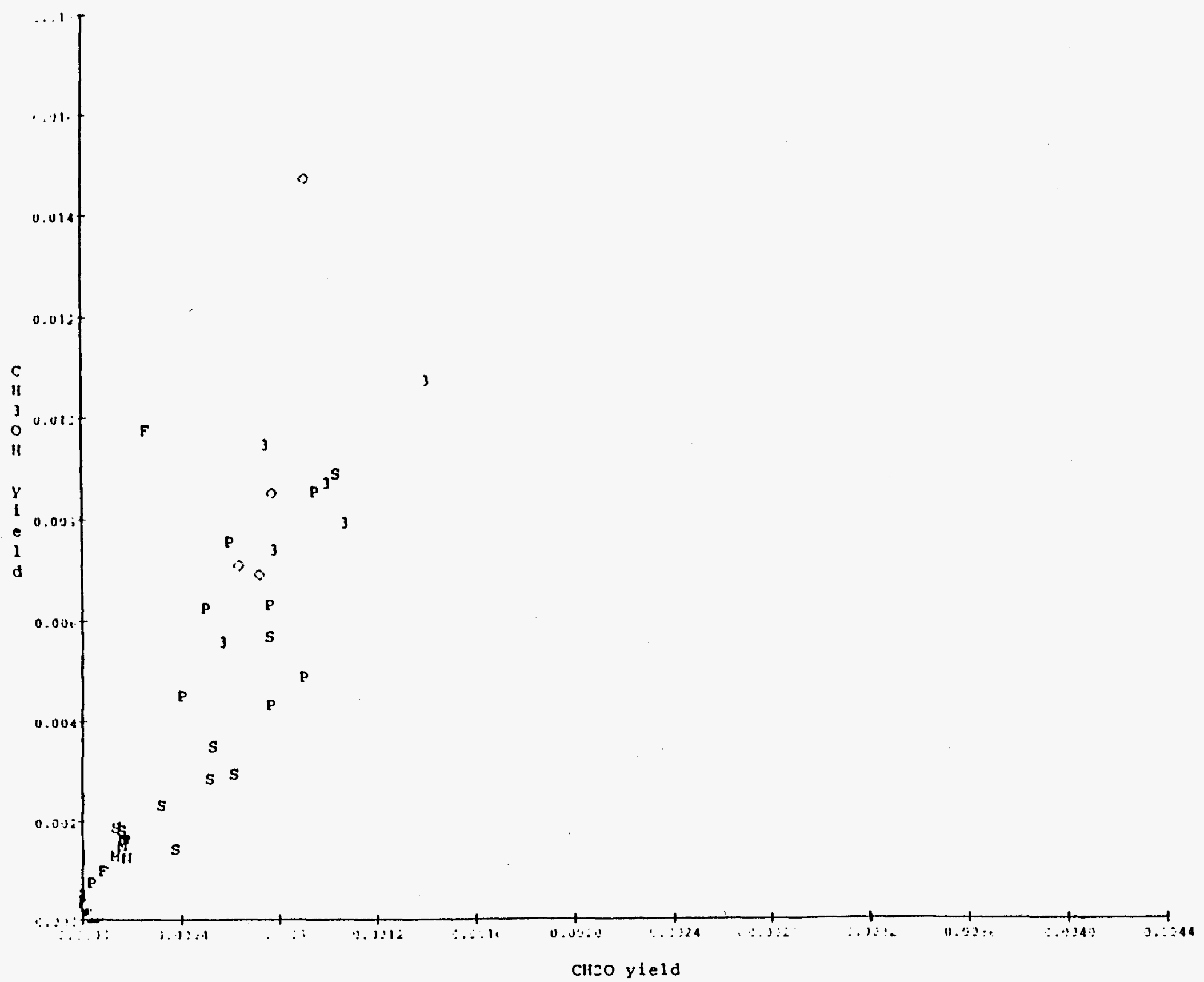

s Slllea oel

N NaJ[Fa (CN) 5 NH3]

M MnAPo

3 [Fe (CN) $5 \mathrm{NH} 3] \cdot]$

- Fe,soda11 to

Fe $[\mathrm{PC}]$

FeC13 $+\mathrm{KHF}^{2}$ 


\subsection{REFERENCES}

(1) Lyons, J. E., Ellis, P. E. and Durante, V. A., Studies in Surface Science and Catalysis, Elsevier, N.Y., 67, 99(1991).

(2) Bedeneev, Gol'denbery, Gorban and Titel'boim, Kinet. Katal. (Trans) 29, 7 (1988); 24, 14; $29,291,25,297$.

(3) Ritz, and Baker, Ind Eng. Chem. Res., 30, 2287 (1991).

(4) U.S. Patent 4,918,249 to Sun Company, Inc.

(5) Gesser, H., et.al., Petr Div. Prep 36, 160 (1991)

(6) Gesser, H., et.al., Appl. Catal, 57, 45 (1990)

(7) Gesser, H., et.al., Comb. Flame 79216 (1990)

(8) Gesser, H., et.al., Ind. Eng. Chem. Res. 27252 (1988)

(9) Burch, R., et.al., I Chem. Soc. Tar Trans. 85, 3561 (1989).

(10) Danen, W. Unconventional Gas Recovery Contractors Review Meeting, U. S. DOE, Morgantown, WV, 7/28/87.

(11) Lignola, P. G. and Reverchon, E., Prog. Energy Combust. Sci. (1987) 14,75; S. I. Ahonkhai and M. H. Back, Can. I. Chem. (1978) 66, 578; J. E. Baggott, et. al., I. Phys. Chem. (1987) 91, 3386.

(12) Yarlagadda, P. S. et. al., Ind. Eng. Chem. Res. (1988) 27, 252.

(13) Kastanas, G. N., et. al., JCS, Chem. Commun. (1988) 1298.

(14) Satterfield, C. N. and Reid, R. C., I. Chem. Eng. Data, (1961), 6, 302; L. F. Albright, et. al., Ind. Eng. Chem., Proc. Des. Dev. (1977) 16, 271.

(15) Durante, V.A., Seitzer, W. H., Walker, D. W., and Lyons, J. E., Presented at the PACIFICHEM Symposium on Methane Oxidation, December 20, 1989.

(16) Wiberg, K. B., "Oxidation in Organic Chemistry", Academic Press, (1965) 69; Groves, J. T., and McClusky, G. A., Biochem. and Biophys. Res. Commun., (1978) 81,154; Ellis, P. E. and Lyons, J. E., Catalysis Letters (1989). 


\subsection{REFERENCES (Cont'd)}

(17) Szostak, R. and Thomas, T. L., ICS Chem. Commun. (1986), 113; G. Calis, et.al., Zeolites (1987) 7,319.

(18) Anderson, J. R. and Tsai, P., ICS. Chem. Commun. (1987), 1435.

(19) Albright, L. F. et. al., Ind. Eng. Chem., Proc. Des. Dev. (1977) 16, 275.

(20) Fejes, P., et. al, Act. Phys. Chem. (1978) 24;119; L. R. M. Martens, et. al., "Preparation of Catalysts IV", B. Delmon ed, (1987), Elsevier, Amsterdam, p. 531.

(21) Gesser, H., Energy and Fuels, 5, 423 (1991)

(22) McDaniels, et. al., J. Catal. $\underline{82}, 98$ (1983); $\underline{82, ~} 110$ (1983); $\underline{82}, 118$ (1983).

(23) Lakin, and Sengupta, Can. J. Chem., 69, 35 (1991).

(24) Boreskov (G.K.) et.al., Kinet Katal (1970) 


\subsection{CONCLUSIONS}

\subsection{THE SUPRABIOTIC CONCEPT}

At the conclusion of our work on the Cooperative Agreement it is instructive to re-visit the Suprabiotic concept and evaluate catalyst performance relative to this idea. As we first envisioned a suprabiotic catalyst, it was one which could operate in the absence of costly stoichiometric co-reductants using only air to oxidize an alkane to an alcohol but in some respects mimic biology in that the active intermediates would be similar. It appears as though the active intermediate which oxidizes alkanes in both Cytochrome $\mathrm{P}-450$ and methane monooxygenase is a ferryl species, $\mathrm{Fe}=\mathrm{O}$. We devised a possible conceptual route to active ferryl using only iron, air and substrate, Figure 1.

Prior to our work no success had been achieved in attempting to oxidize alkanes to alcohols with only air or oxygen in this manner. Many attempts had been made using porphyrinato complexes both with and without bulky groups which would prevent $\mu$-oxo dimer formation, Figure if. The only successful alkane oxidations using porphyrinato complexes were achieved using costly single atom donor oxidants such as iodosylbenzene, hypochlorites, or hydroperoxides.

\subsection{MOLECULAR COMPLEXES FOR HOMOGENEOUS LIOUID PHASE CONVERSIONS}

The problems associated with this system for air-oxidations of alkanes to alcohols were many. First, although formation of $\mu$-oxo dimer from the iron(II) precursor is detrimental, $\mu$-peroxo dimer formation is crucial. Secondly, access of the alkane to the active center must be good. Thirdly, the alkyl radical formed by $\mathrm{C}-\mathrm{H}$ bond homolysis should remain in the coordination sphere until rebound to the alcohol occurs. Fourth, the alcohol should be expelled as quickly from the coordination sphere as possible to prevent over-oxidation. Next, $\mu$-oxo dimer formation must be reversed or prevented. Finally, the iron(III)/(II) reduction potential must be high enough to continuously regenerate active $\mathrm{Fe}$ (II) from the pool of $\mathrm{Fe}$ (III) even in the presence of molecular oxygen. In addition, the ligand system must be robust to oxygenation, and must not have electrons readily available to reduce the high oxidation state ferryl before it can oxidize the alkane. The kind of porphyrin complexes available prior to our work did none of these things and consequently were inactive catalysts.

Using perhaloporphyrin complexes we: a) stabilized active iron-oxygen centers to both of the kinds of decay referred to above, b) provided a lipophilic environment to attract the alkane to the active site, c) provided a hydrophilic environment to expel alcohol from the coordination sphere, d) tuned the redox potential of the metal center so that $\mu$-oxo dimer was no longer inactive and so that iron(II) species could be continually regenerated in an oxidizing environment. Figure 2 suggests how our perhaloporphyrins may act as a suprabiotic catalyst for converting alkanes to alcohols.

We have discussed how the electronic and steric environment about the oxidation-active metal center affects the rate and selectivity of alkane oxidation in both homogeneous liquid phase oxidations as well as heterogeneous catalytic vapor phase reactions. Reaction rate seems to be highly dependent 


\section{FYPOTHETICAL REACTION PATHWAY FOR A SUPRABIOTIC CATALYTIC ALKANE OXIDATION}

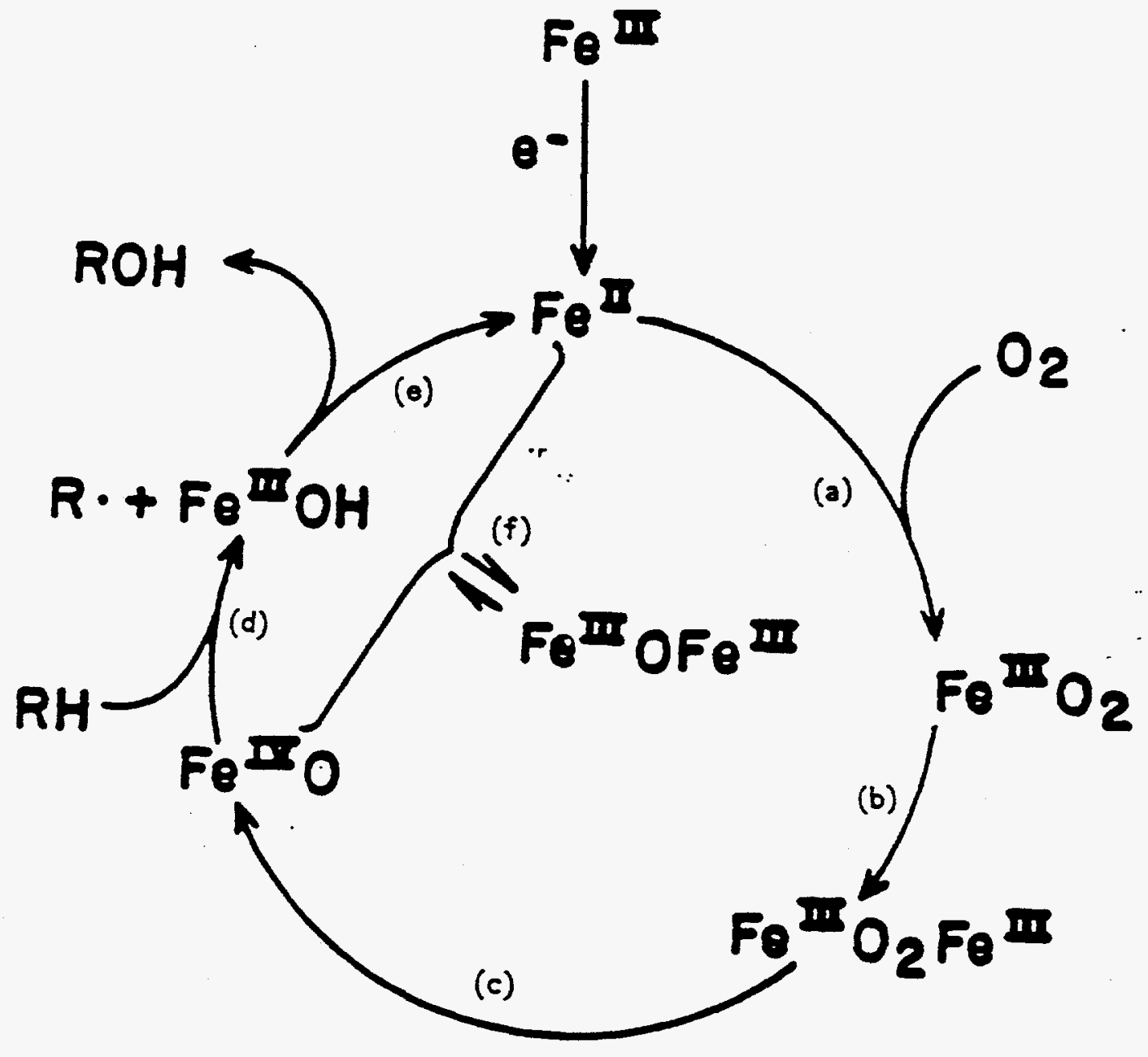

$F e=F Q=$ PORPHYRINATO IRON 
FIGURE 2

\section{PROPERTIES OF A SUPRABIOTIC CATALYST}

- COOPERATIVE IRON (II) SITES

$2 \mathrm{Fe}^{\mathrm{II}}+\mathrm{O}_{2} \longrightarrow \mathrm{Fe} \mathrm{II}_{2} \mathrm{Fe}^{\mathrm{II}} \longrightarrow \mathrm{Fe}^{\mathrm{IVO}}$

- LIPOPHILIC ENVIRONMENT *

$\mathrm{Fe}^{\mathrm{INO}}+\mathrm{RH} \longrightarrow \mathrm{Fe}_{\mathrm{RH}} \mathrm{RH}_{\mathrm{R}} \longrightarrow \mathrm{FeOH}_{\mathrm{R}}$

- HIGH Fe ${ }^{I I} / \mathrm{Fe}^{I}$ REDUCTION POTENTIAL

$\mathrm{Fe}_{\mathrm{R}}^{\mathrm{II}} \mathrm{OH} \longrightarrow \mathrm{Fe} \longrightarrow \mathrm{ROH}$

* HYDROPHOBIC ENVIRONMENT

- SUPPRESSED $\mu$-OXO FORMATION

$\mathrm{Fe}^{I V} \mathrm{O}+\mathrm{Fe}^{I I} \rightleftharpoons \mathrm{Fe}^{I I I} \mathrm{OFe}^{I I I}$

- STABILITY TO OXIDATION

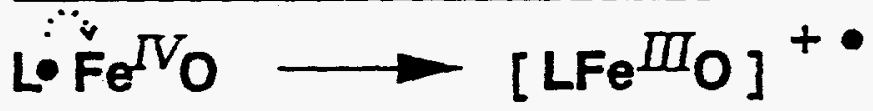

$\mathrm{Fe}^{\mathrm{INO}}+\mathrm{LFe}^{x} \longrightarrow \mathrm{Fe}^{I I}+\mathrm{OLFe}^{x}$ 
on proper tuning of redox potentials. In the liquid phase we have achieved unprecedented reactionrates and have electrochemical evidence that much higher rates can still be achieved. In the vapor phase we have shown the need for proper tuning of reduction potential but we must synthesize catalysts which have far greater reduction potentials than those which we currently have if we are to achieve the rates that will ultimately be necessary.

While steric effects influence rate as well, we are finding that their prime importance is in reaction selectivity. Ejecting formed alcohol from the coordination sphere is of great importance as we have seen in both homogeneous and heterogeneous alkane oxidation. Although our greatest gains to date are in tuning the redox potential for high activity, and although there is still room for significant improvement here, one should concentrate in the future on those aspects of catalyst structure that will effect selectivity to alcohol. Of all of these structural factors, the most important is hydrophobicity of the ligand or surface environment. Our current designs address these point and we are confident that we will continue to make significant gains in both reaction rate and selectivity until all of the light alkanes can be smoothly converted to alcohols by direct, practical, air-oxidations.

In the Topical Reports on the Cooperative Agreement, we have mentioned the exciting new results which are enabling us to convert light alkanes to alcohol-rich oxygenates for use as fuels and chemicals. As we progressed in this research, the national climate mandating oxygenates in fuels for clean air and the growing need for alternate fuels added further impetus in addition to the economic incentive for generating high octane oxygenates from inexpensive light alkanes found in natural gas and in light refinery streams being rejected from gasoline because of emission restrictions. We have also noted that we are getting close to new catalytic technology which will enable us to produce-TBA from isobutane. The commercial impact such technology will have on the production of MTBE, the largest growing fuel oxygenate could be very great. In addition, it is being realized that alcohols such as TBA and IPA will become important additions to the gasoline pool not only as cosolvents for methanol and ethanol but in mixtures with ethers. Sun has participated with several other oil companies in helping to procure recent EPA approval of oxygenate compositions containing TBA or IPA together with MTBE and other ethers to meet oxygenate requirements in transportation fuels. The oxygenates which we are producing directly from alkanes and oxygen using our novel catalytic chemistry will have immediate utility in an emerging new energy scenario.

At the end of our work on the Cooperative Agreement it is well to step back and look not only at our catalytic accomplishments and their relevance to the national energy picture, but also to assess the strides made in understanding what is clearly a new class of oxidation catalysts. Only through understanding the mechanism of the action of this new class of catalysts will we be able to fully utilize them in producing valuable products.

As we have noted in past reports, we developed the concepts leading to the preparation of the catalysts used in this program from considerations of how enzymatic systems such as methane monooxygenase are able to convert light alkanes to alcohols, Figures 3. It became clear that high oxidation state metal oxo's might well be the key intermediates which reacted with the alkane to produce the alcohol. One of the practical problems inherent in mimicking this approach is the need for expensive coreductants (sources of electrons) to take the role supplied by NADH in the biological systems. We asked the question whether it would be possible to adjust the redox potential of simple 


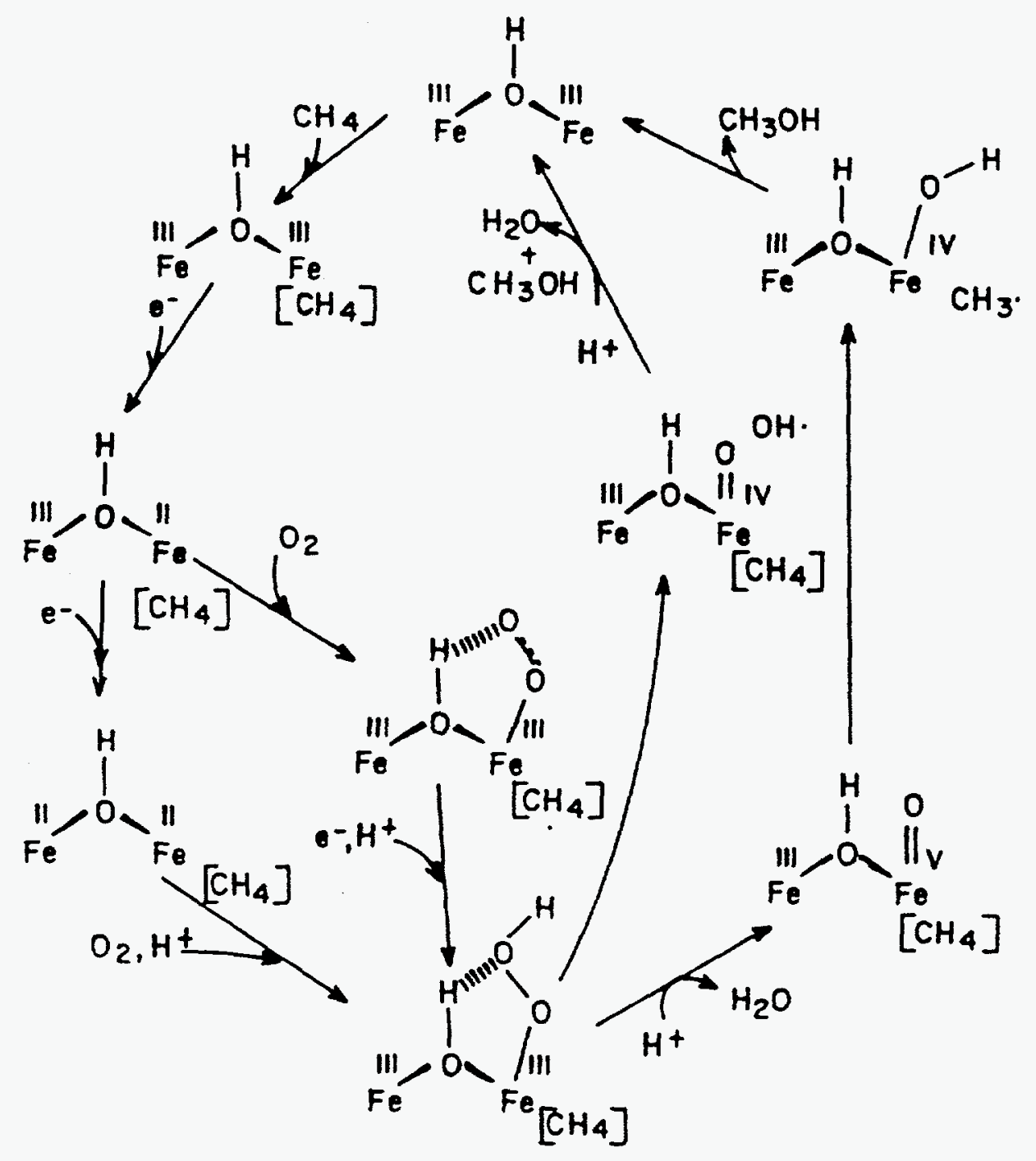


metal complexes modeled after the biological systems such that they could do something heretofore not accomplished - convert alkanes to alcohols through high oxidation state metal oxo intermediates using only air or oxygen without the need for stoichiometric consumption of expensive coreductants. The mechanism which we envisioned for accomplishing this is outlined schematically in Figure 1 and the technique used to adjust the redox potential was to make complexes in which there was a high degree of electron withdrawal from the active iron center. Additionally we wished to create molecular environments in which two iron centers could cooperate in reductively splitting the $0-0$ bond without forming inactive $\mu$-oxo dimers.

The finding that by steadily increasing the degree of halogenation and therefore the amount of electron withdrawal from the iron center, one steadily increases both the catalytic air-oxidation activity and the reduction potential of the iron complex, shows that our catalytic chemistry responds to the same factors that biomimetic catalysts do - with one important exception - our catalysts use only air or oxygen as the oxidant. This is good circumstantial evidence that the reaction may be going through the originally postulated reaction mechanism.

Subsequent findings that proximate diiron centers are superior to single iron sites in both liquid phase reactions catalyzed by polyoxometallates as well as gas phase reactions catalyzed by framework substituted solid catalysts infer a special activity which may be related to the ability of these systems to bind and activate oxygen across both iron sites. These catalysts are generally used at relatively high temperatures so that intermediates within the catalytic cycles liberate alkyl radicals either to a small extent or, in some instances, as a major reaction pathway and this allows radical chain processes to compete with catalysis.

In summary, it is gratifying to have obtained a large amount of evidence which is consistent with our original hypotheses. This shows that, although we are still learning much about the details of the reaction pathways involved, we are indeed on the right track in observing truly novel chemistry and that continued extrapolation of the principles which are being uncovered will continue to produce catalysts with increasingly superior properties for conversion of light alkanes to fuel oxygenates.

\subsection{FRAMEWORK SUBSTITUTED ZEOLITES}

Now that we have established structure-reactivity relationships which correlate both the $\mathrm{Fe}(\mathrm{III}) / \mathrm{Fe}$ (II) reduction potential and the steric environment about the metal center with catalytic activity we are beginning to design ligand systems which have electronic and steric properties that will produce superior catalysts. Paramount among the considerations now being employed in addition to chemical characteristics are economic characterisitcs as well. For instance, the cost of raw materials and the molecular weight of the macrocycle are two factors which will be critical in the design of inexpensive catalysts for commercial production of fuels. We are indeed fortunate that iron provides the most active catalyst rather than a costly noble metal. 
We have now learned how to tune the redox potential of metalloprophyrin catalysts toward high oxidation activity. In addition we have learned how to prepare active, thermally stable polyoxometallates with proximate metal centers, but these catalysts do not have the good low-temperature activity of the perhaloporphyrin systems and produce a less alcohol-rich product. We realize that the redox potentials of the proximate metals must be further tuned $[M(I I)+e-M(I I)]$ to make superior catalysts of this type. Similarly we have learned how to prepare active iron sodalite and manganese aluminophosphate catalysts for vapor phase oxidation of methane to methanol which have proximate metal centers. The sodalite framework, however may impede the ablility of substituted iron to achieve the full range of oxidation states desired for catalytic oxidation. This may be the reason that we need extra-framework iron (Fex[Fe]SOD) for high activity. What may be needed for a heterogeneous catalyst which exhibits good low-temperature alkane oxidation activity is proximate metal centers each of which have a high reduction potential. Much of the emphasis in research on the Cooperative Agreement focused on catalysts containing binuclear metal centers having high $\mathrm{M}(\mathrm{III}) / \mathrm{MII})$ reduction potentials to enable them to reductively couple dioxygen.

An active suprabiotic Catalyst should therefore have: (a) high reduction potential (to create oxygen binding sites) and (b) proximate iron centers (for oxygen cleavage), Fig. 4.

We have made substantial progress in the area of increasing the activity of heterogeneous catalysts for methane and ethane oxidations. In our laboratories we have recently addressed the structural limitations that some zeolites impose on $\mathrm{M}(\mathrm{III}) / \mathrm{M}(\mathrm{II})$ transformations by incorporating large metal concentrations in aluminophosphates. A manganese aluminophosphate in which both $\mathrm{Mn}$ (III) and $\mathrm{Mn}$ (II) may be incorporated allows us to activate methane for catalytic conversion to methanol at temperatures fully $35^{\circ} \mathrm{C}$ lower than are required for Fex[Fe]SOD and over $50^{\circ} \mathrm{C}$ lower than is necessary for thermal oxidation. A number of electronically tuned binucleating macrocycles have been synthesized and supported on hydrophobic surfaces during the later stages of the Cooperative Agreement.

\subsection{CATALYST SYNTHESIS AND TESTING}

We have found highly active catalyst systems which give unprecedented rates of activation of light alkanes to form oxygenates in liquid and vapor phase. Results of our research confirms many of our intial ideas and suggests that there is a high probability of success in achieving practical processes for alkane conversions based on the new science and technology which we have generated. Recent success in making potentially cheaper and more active catalysts is particularly important as we enter the proof-of-concept stage for a process to make tert-butyl alcohol from $\mathrm{C}_{4}$ gas components. Continued research is likely to result in future process technology for $\mathrm{C}_{3}-\mathrm{C}_{3}$ gas components as well.

During the Cooperative Agreement we have made a number of heterogeneous catalysts and screened them for methane oxidation to methanol. We synthesized, characterized and tested several [M]Pentasil compounds namely, [Fe]ZSM-5, [V]ZSM-5, and [Ru]ZSM-5 and determined that metal contents above $3 \%$ were not obtained and catalytic activity and selectivity to methanol were not high. We were unable to make [Mn]ZSM-5 after several attempts. We synthesized, characterized and tested several [M]Sodalite catalysts as well. We were able to synthesize the iron substituted [Fe]Sod which had over $15 \%$ iron in the sodalite framework and this gave much higher activity and produced 
FIGURE 4
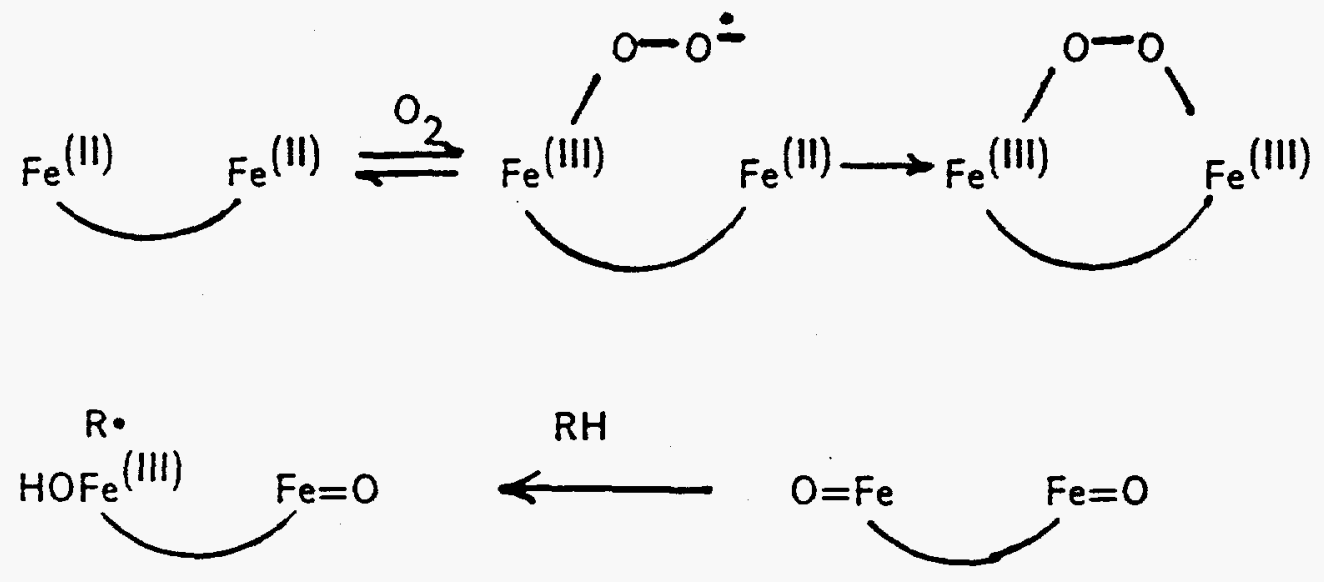
methanol in better selectivity. We believe that the high iron loading must place iron atoms in close proximity and these proximate iron sites might bind dioxygen in a cooperative manner. We found that incorporating more iron into the exchange sites of [Fe] Sod gave an even better catalyst (Fe)x[Fe]Sod. Activity was further enhanced by treatment of this catalyst with sodium azide. We failed to make the manganese analog, $[\mathrm{Mn}]$ Sod after many attempts. We were able to make a manganese exchanged iron substituted sodalite, (Mn)x[Fe]Sod, but it was inferior to (Fe)x[Fe]Sod. The final series on our program was designated [M]Sieve. These materials were the framework metal substituted aluminophosphates (ALPO's). In this case we were able to incorporate both iron and manganese into aluminophosphate frameworks in high concentrations to give in one instance FAPO-11 - a known material, and in the other case, MnAPO-X - a crystalline manganese ahuminophosphate of as yet unknown structure. We have completed both of these syntheses have characterized the materials and have tested the manganese ALPO for methane oxidation. MnAPO-X has incorporated $8 \%$ by weight of $\mathrm{Mn}$ and it's activity is comparable to that of $(\mathrm{Fe}) \times[\mathrm{Fe}]$ Sod under mild conditions This represents the first time we have been able to "tame" a manganese center to give a significant yield of methanol rather than to yield only combustion products. The FAPO-11 will be tested during the next quarter.

The series of catalysts which were designated to be prepared during the second year of PHASE III activity were catalysts having the highest number of binuclear active metal sites possible. We have synthesized new mixed metal oxides of the perovskite, $\mathrm{MMO}_{3}$ stoichiometry which has much higher metal-oxygen ratios than those of the framework-substituted zeolites discussed above. Another reason for our interest in perovskite-like materials having a high metal-oxygen ratio was the mobility of lattice oxygen in such structures. We believe that low temperature oxygen mobility is characteristic of systems which are expected to demonstrate lattice oxygen involvement in alkane oxidations, perhaps through creation of metal-oxo intermediates at redox active metal sites in these structures. Another important feature which a low temperature oxidation catalyst should have is the ability to regenerate lower oxidation states capable of activating molecular oxygen. Of particular interest in this regard are the manganites in which the oxidation state of one of the metals in the binuclear site can be affected by the nature of the second metal. We have synthesized catalysts of all of these types and many were active catalysts but all gave predominant combustion and no methanol.

Throughout the second and third year of the Cooperative Agreement, we continued to screen FAPO, MnAPO and CoAPSO catalysts which demonstrate interesting low temperature heterogeneous catalytic activity for vapor phase oxidation of methane and ethane. We also assessed the kinetic modelling study which we performed on hypothetical catalytic and non-catalytic oxidations which proceeded via generation of gas-phase methyl radical. These studies combined with supporting experiment convince us that it is unlikely that gas phase methyl radicals, whether generated thermally in small amounts or catalytically in greater quantity, will provide sufficiently selective high yield pathways to be commercially attractive in the future fuel scenarios that we envision.

We must add two important criteria to our heterogeneous catalyst design in order to achieve practical systems for converting methane or ethane to alcohols in high yield. Figure 2 shows the suprabiotic criteria for liquid phase oxidation of an alkane to an alcohol. The first criterion indicates that low oxidation state metal centers must cooperate to reductively bind dioxygen. In solution, 
mononuclear macrocyclic complexes can migrate to a single oxygen site. Surface migration might not be as easy a process and segregated sites will not suffice. We have made a large number of catalysts which have proximate metal sites, however the ease of reduction of these sites (or the number of reduced sites in proximity) might not be sufficient to reductively bind dioxygen. A criterion for future catalysts will be binuclear iron centers and a number of these have been designed and are being synthesized at the present time.

We have discussed the need for high reduction potential but another criterion which has been missing in our catalysts is that of a hydrophobic environment. Future catalysts will be constructed so that the oxidation-active metal center is in a hydrophobic site which attracts alkane, encourages surface reaction and rapidly expels alcohol as product. The catalysts of the future will operate at lower temperature, will have a low contribution of vapor phase radical reaction and operate via surface reactions to a greater extent than those we have generated in the past.

During the third and fourth year of the Coopeative Agreement, we have concentrated on the synthesis of metal complexes of electron defficient binucleating macrocycles that can be supported on hydrophobic cariers. This work is detailed in Topical Reports IV and VI. 


\subsection{SUN PATENTS AND PUBLICATIONS DURING THE COURSE OE THE COOPERATIVE AGREEMENT}

\subsection{PATENTS ISSUED OR PUBLISHED}

(1) P. E. Ellis, Jr., J. E. Lyons, and H. K. Myers, Jr., "Hydrocarbon Oxidations Catalyzed by Nitride-Activated Metal Coordination Complexes", U.S. Patent 4,895,680, issued Jan. 23, 1990.

(2) P. E. Ellis, Jr., J. E. Lyons, and H. K. Myers, Jr., "Hydrocarbon Oxidations Cataiyzed by Azide-Activated Metal Coordination Complexes", U.S. Patent 4,895,682, issued Jan. 23, 1990.

(3) P. E. Ellis, Jr. and J. E. Lyons, "Alkane Oxidation Process", U.S. Patent 4,898,989, issued Feb. 6, 1990.

(4) P. E. Ellis, Jr., J. E. Lyons, and H. K. Myers, Jr., "Hydrocarbon Oxidations Catalyzed by Iron Coordination Complexes Containing A Halogenated Ligand", U.S. Patent 4,900,871, issued Feb. 13, 1990.

(5) J. E. Lyons, P. E. Ellis, Jr., W. A. Langdale, and H. K. Myers, Jr., "Method of Preparing Heteropoly Acid Catalysts", U.S. Patent 4,916,101, issued April 23, 1990.

(6) V. A. Durante, D. W. Walker, S. M. Gussow, and J. E. Lyons, "Silicometallate Molecular Sieves and Their Use as Catalysts in Oxidation of Alkanes", U.S. Patent 4,981,249, issued April 23, 1990.

(7) P. E. Ellis, Jr. and J. E. Lyons, "Chromium Halogenated Coordination Complexes For The Oxidation of Butane to Methyelthylketone," U.S. Patent 4,970,348, issued Nov. 13, 1990.

(8) P. E. Ellis, Jr. and J. E. Lyons, "Alkane Oxidation Catalysts, " U.S. Patent 5,091,354, issued Feb. 25, 1992

(9) P. E. Ellis, Jr., J. E. Lyons, and H. K. Myers, Jr., "Hydrocarbon Oxidations Catalyzed by Azide-Activated Metal Coordination Complexes," U.S. Patent 5,093,491, issued March 3, 1992.

(10) P. E. Ellis, Jr. and J. E. Lyons, "Cyano and Polycyanometalloporpyrins as Catalysts for Alkane Oxidation," U.S. Patent 5,118,886, issued June 2, 1992.

(11) P. E. Ellis, Jr. and J. E. Lyons, "Nitrated Metalloporphyrins as Catalysts for Alkane Oxidation," U.S. Patent 5,120,882, issued June 9, 1992. 


\subsection{PATENTS ISSUED OR PUBLISHED (Cont'd)}

(12) J. E. Lyons and P. E. Ellis, Jr., "Decomposition of Organic Hydroperoxides," U.S. Patent 5,120,886, issued June 9, 1992.

(13) V. A. Durante, D. W. Walker, S. M. Gussow, J. E. Lyons and R. C. Hayes, "Catalytic Oxidation of Alkanes", U.S. Patent 5,132,472, issued July 21 (1992).

(14) P. E. Ellis, Jr., and J. E. Lyons, "Cyano- and Polycyanometallo-Porphyrins as Catalysts for Alkane Oxidations," U.S. Patent 5,212,300, issued May 18, 1993.

(15) J. E. Lyons, V. A. Durante and D. W. Walker, "Chromia on Metal Oxide Catalysts for the Oxidation of Methane to Methanol," U.S. Patent 5,220,080 issued June 15, 1993.

(16) P. E. Ellis, Jr., and J. E. Lyons, "Metal Phthalocyanine Oxidation Catalysts," U.S. Patent $5,254,740$, issued October 19, 1993.

(17) P. E. Ellis, Jr., and J. E. Lyons, "Nitrated Metalloporphyrins as Catalysts for Alkane Oxidation," U.S. Patent 5,280,115, issued January 20, 1994.

(18) S. N. Shaikh, P. E. Ellis, Jr., and J. E. Lyons, "Oxidation of Hydrocarbons Over Ordered Arrays of Heteropolyacids and Polyoxoanions on Graphite," U.S. Patent 5,334,780, issued August 2, 1994.

(19) V. A. Durante, J. E. Lyons, D. W. Walker, and B. K. Marcus, "New Manganese Catalyst for Light Alkane Oxidation," U. S. Patent 5,345,001, issued September 6, 1994.

(20) J. E. Lyons, V. A. Durante and D. W. Walker, "Chromia on Metal Oxide for Oxidation of Alkane to Alcohol," U.S. Patent 5,345,010, issued September 6, 1994.

(21) J. E. Lyons and P. E. Ellis, Jr., "Decomposition of Organic Hydroperoxides with Nitrated Porphyrin Complexes," U.S. Patent 5,345,008, issued September 6, 1994.

(22) P. E. Ellis, Jr. and J. E. Lyons, "Metal Phthalocyanine Catalysts," U.S. Patent 5,354,857, issued October 11, 1994.

(23) P. E. Ellis, Jr. and J. E. Lyons, "Cyano- and Polycyanometalloporphyrins as Catalysts for Alkane Oxidation," U.S. Patent 5,382,662, issued January 7, 1995. 


\subsection{PUBLICATIONS}

(1) J. E. Lyons and G. W. Parshall, "Catalysis for Industrial Chemicals," Catal. Today, 22, 313 (1994).

(2) J. E. Lyons, P. E. Ellis, Jr., and H. K. Myers, Jr., "Halogenated Metalloporphyrin Complexes as Catalysts for Selective Reactions of Alkanes with Molecular Oxygen," I. Catal. (in press).

(3) W. P. Schaefer, P. E. Ellis, Jr., J. E. Lyons and S. N. Shaikh, "A $\mu$-oxo-bis(Iron(III)porphyrin) Complex," Acta. Cryst. (in press).

(4) T. P. Wijesekera, J. E. Lyons, and P. E. Ellis, Jr., "Perfluoroalkylporphyrin Complexes as Active Catalysts for the Reaction of Isobutane with Oxygen and the Decomposition of tert-Butyl Hydroperoxide," Catal. Lett. (in press).

(5) J. E. Lyons and G. W. Parshall, "Catalysis for Industrial Chemicals," Research Needs Assessment Report to U.S. Department of Energy, DE-FG02-92ER30201, (1993).

(6) R. R. Chianelli, J. E. Lyons and G. A. Mills, "Liquid Transportation Fuels from Petroleum Coal, Residual Oil and Biomass," Research Needs Assessment Report to U.S. Department of Energy, DE-FG02-92ER30201, (1993).

(7) P. E. Ellis, Jr., J. E. Lyons and S. N. Shaikh, "Novel Iron Meso-Nitroporphine Complexes for the Catalytic Reaction of Alkanes with Molecular Oxygen," Catal. Lett., 24, 79 (1994).

(8) H. L. Chen, P. E. Ellis, Jr., T. P. Wijesekera, T. E. Hagan, S. E. Groh, J. E. Lyons and D. P. Ridge, "Correlation Between Gas Phase Electron Affinities, Electrode Potentials and Catalytic Activities of Halogenated Metalloporphyrins", L Amer. Chem. Soc., 116, 1086 (1994).

(9) J. E. Lyons and P. E. Ellis, Jr., Reactions of Alkanes with Dioxygen: Toward Suprabiotic Systems, Metalloporphyrins in Catalytic Oxidations, R. S. Sheldon, Ed. Marcel Dekker, N.Y., pp 297-324 (1994).

(10) P. E. Ellis, Jr., and J. E. Lyons, "Commentary on 'Carboxylation of Propane with CO to Butyric Acids By Pd(II)/Cu(I) Based Catalysts'," Chemtracts, 5 , 298 (1993).

(11) R. E. Marsh, W. P. Schaefer, J. A. Hodge, M. E. Hughes, H. B. Gray, J. E. Lyons and P. E. Ellis, Jr., "A Highly Solvated Zinc(II)Tetrakis(pentafluorophenyl- $\beta$-octabromoporphyrin", Acta. Cryst. Sec. C, C49, 1339 (1993). 
(12) L. E. Henling, W. P. Schaefer, J. A. Hodge, M. E. Hughes, H. B. Gray, J. E. Lyons, and P. E. Ellis, Jr., "Copper(II) and Nickel(II)Tetrakis(pentafluorophenyl)- $\beta$-octabromoporphyrins", Acta. Cryst., Sec. C, C49 (1993).

(13) W. P. Schaefer, J. A. Hodge, M. E. Hughes, H. B. Gray, J. E. Lyons, P. E. Ellis, Jr. and R. W. Wagner, "Copper(II) Tetrakis(pentafluorophenyl)-octachloroporphyrin" Acta. Cryst. Sec. C, C49, 1342 (1993).

(14) J. E. Lyons, P. E. Ellis, Jr., H. K. Myers, Jr., and R. W. Wagner, "Halogen Substituent Effects On The Catalytic Activity Of Iron Porphyrin Complexes For The Decomposition Of tert-Butyl Hydroperoxide," J. Catal., 141, 311 (1993).

(15) M. T. Klein, A. T. Lapinas, B. C. Gates. C. J. Read, J. E. Lyons and A. Macris, "Cleavage Of Biphenyl Moieties: An Efficient New Reaction Pathway in Catalytic Hydrocracking," L Catal., 137, 504 (1992).

(16) J. E. Lyons, "Light Alkane Conversion Processes - Suprabiotic Catalyst Systems For Selective Oxidation Of Light Alkane Gases To Fuel Oxygenates," Proc. Nat. Gas Res. And Dev Centr Rev. Mtg., DOE/METC - 92/6125, May, 1992, pp. 266-278.

(17) J. E. Lyons, P. E. Ellis, Jr., R. W. Wagner, P. B. Thompson, H. B. Gray, M. E. Hughes, J. A. Hodge, "Air-Oxidation of Light Alkanes by First Row Transition Metals in Macrocyclic Ligand Environments." Preprints of the Petroleum Division, 37307 (1992).

(18) J. E. Lyons, "Selective Oxidation of Hydrocarbons via C-H Bond Activation by Soluble and Supported Palladium Catalysts", Chemical Catalyst News, Engelhard Corporation, May, 1992.

(19) J. E. Lyons and P. E. Ellis, Jr., "Azide Activation of Metallophthalocyanine Complexes for the Catalytic Oxidation of Alkanes in the Liquid Phase," Applied Catalysis. A General, 84, L1-L6 (1992).

(20) J. E. Lyons, P. E. Ellis and V. A. Durante, "Active Metal Oxo Centers for the Selective Catalytic Oxidation of Alkanes", Studies in Surface Science and Catalysis, Elsevier, N.Y. 67, 99 (1991).

(21) J. E. Lyons and P. E. Ellis, Jr., "Selective Low Temperature Hydroxylation of Isobutane By Molecular Oxygen Catalyzed by an Iron Perhaloporphyrin Complex", Catalysis Letters, $\underline{8}, 45$ (1991). 


\subsection{PUBLICATIONS (Cont'd)}

(22) J. E. Lyons, "Suprabiotic Catalyst Systems For Selective Oxidation Of Light Alkane Gases To Fuel Oxygenates," Proc. Nat. Gas Res. And Dev. Contr. Rev. Mtg. DOE/METC 91/6117, Nov. (1990) pp. 276-286.

(23) P. E. Ellis, Jr. and J. E. Lyons, "Selective Air-Oxidation of Light Alkanes Catalyzed by Activated Metalloporphyrins - The Search for a Suprabiotic System", Coordination Chemistry Reviews, 105, 181 (1990).

(24) P. E. Ellis, Jr. and J. E. Lyons, "Selective Air-Oxidation of Alkanes Catalyzed by Halogenated Metalloporphyrins", Preprints of the Division of Petroleum Chemistry, ACS, Vol. 35, No. 2, April (1990).

(25) V. A Durante, D. W. Walker, W. H. Seitzer, and J. E. Lyons, "Vapor Phase Hydroxylation of Methane", Preprints of 3B Symposium on Methane Activation. Conversion and Utilization. International Chemical Congress of Pacific Basin Societies, 23 (1989).

(26) P. E. Ellis and J. E. Lyons, "Halogen Substituent Effects on the Catalytic Activity of Iron Porphyrin Complexes for the Selective Air-Oxidation of Alkanes in the Liquid Phase", Catalysis Letters, 3, 389 (1989).

(27) P. E. Ellis and J. E. Lyons, "Effect of Fluorination of the Meso-Phenyl Groups on Selective Tetraphenylporphyrinatoiron(III)-Catalyzed Reactions of Propane With Molecular Oxygen, I Chem. Soc. Chem. Commun., 1315 (1989).

(28) P. E. Ellis and J. E. Lyons, "Effect of Fluorination of the Meso-Phenyl Groups on SelectiveTetraphenylporphyrinatometal(III)-Catalyzed Reactions of Isobutane with Molecular Oxygen", J. Chem. Soc. Chem. Commun., 1189 (1989).

(29) P. E. Ellis and J. E. Lyons, "Effect of Axial Azide on the Selective, Low Temperature Metalloporphyrin-Catalyzed Reactions of Isobutane with Molecular Oxygen", I Chem. Sec. Chem Commun., 1187 (1989).

(30) J. E. Lyons, P. E. Ellis, Jr., and V. A. Durante, "Catalytic Conversion of Light Alkanes", Proceedings of the Natural Gas R\&D Contractors Review Meeting, DOE/METC-89/6103, 164 (1989). 
Manoj V. Bhinde

Thomas W. Bierl

Kevin A. Boyer

Paul T. Bowden

Rogar G. Carter

Eugene L. Coggins

Christine R. Cox

Vincent A. Durante

Paul E. Ellis, Jr.

Harry B. Gray

Allen W. Hancock

Chen-Shi Huang

Joseph S. Klein

Jay Labinger

Wayne A. Langdale

Guang-Huei Lee

James E. Lyons

Ronald A. Merrill

Floyd A. Moore

Harry Myers, Jr.

Margaret Nemet

Kevin S. Norbash

David S. Scott

Walter A. Seitzer

Shahid N. Shaikh

Wilbert Tsao

Tilak P. Wijesekera 